Portland State University

PDXScholar

Dissertations and Theses

Dissertations and Theses

Summer 7-12-2017

\title{
Exploring the Positive Utility of Travel and Mode Choice
}

Patrick Allen Singleton

Portland State University

Follow this and additional works at: https://pdxscholar.library.pdx.edu/open_access_etds

Part of the Transportation Commons

Let us know how access to this document benefits you.

Recommended Citation

Singleton, Patrick Allen, "Exploring the Positive Utility of Travel and Mode Choice" (2017). Dissertations and Theses. Paper 3780.

https://doi.org/10.15760/etd.5664

This Dissertation is brought to you for free and open access. It has been accepted for inclusion in Dissertations and Theses by an authorized administrator of PDXScholar. Please contact us if we can make this document more accessible: pdxscholar@pdx.edu. 
Exploring the Positive Utility of Travel and Mode Choice

\title{
by
}

Patrick Allen Singleton

\begin{abstract}
A dissertation submitted in partial fulfillment of the requirements for the degree of
\end{abstract}

\author{
Doctor of Philosophy \\ in \\ Civil and Environmental Engineering
}
Dissertation Committee:
Kelly J. Clifton, Chair
Jennifer Dill
Liming Wang
Cynthia D. Mohr

\section{Portland State University}

2017 
(C) 2017 Patrick Allen Singleton 


\begin{abstract}
Why do people travel? Underlying most travel behavior research is the deriveddemand paradigm of travel analysis, which assumes that travel demand is derived from the demand for spatially separated activities, traveling is a means to an end (reaching destinations), and travel time is a disutility to be minimized. In contrast, the "positive utility of travel" (PUT) concept suggests that travel may not be inherently disliked and could instead provide benefits or be motivated by desires for travel-based multitasking, positive emotions, or fulfillment. The PUT idea assembles several concepts relevant to travel behavior: utility maximization, motivation theory, multitasking, and subjective well-being.

Despite these varied influences, empirical analyses of the PUT concept remain limited in both quantity and scope. There is a need for more fundamental development and classification of the PUT idea and its multifaceted nature. The wide variety and quality of ways to measure PUT attributes are further research challenges. Additionally, few studies investigate both major aspects of the PUT concept-travel activities and travel experiences—simultaneously. Finally, research is only beginning to examine empirical associations between PUT measures and travel behaviors such as mode choice. This dissertation addresses many of these gaps in conceptualizing, measuring, and modeling the PUT concept.
\end{abstract}

First, a literature review strengthens the definition, classification, and empirical support for a PUT, defined as "any benefit(s) accruing to a traveler through the act of traveling." The two primary PUT categories are travel activities (travel-based multitasking) and travel experiences (travel subjective well-being), and the most useful PUT measures involve gathering self-reported assessments of these topics. Based on this 
review, an online questionnaire is designed and administered to nearly 700 commuters in the Portland, OR, region. The survey includes detailed questions about commute mode choice, activity participation, travel usefulness, positive emotions and fulfillment, and travel liking for a recent home-to-work trip.

Next, these PUT measures are empirically examined using factor analyses, finding groupings of activities and common unobserved constructs of hedonic ("Distress," "Fear," "Attentiveness," "Enjoyment") and eudaimonic ("Security," "Autonomy," "Confidence", "Health") subjective well-being. Many of these factors exhibit large variations among travel modes - walking and bicycling commuters are the most satisfied and appear to value time spent exercising - and are predicted (somewhat less strongly) by other trip and traveler characteristics in ordered logit regression and structural equation models.

Finally, integrated choice and latent variable models are estimated to examine relationships between measures of the PUT concept and commute mode choice. This is made possible by the unique dataset that collects PUT measures for not only the chosen mode but also modal alternatives. Measures of travel-based multitasking are significantly related to mode choice, suggesting people may be doing things more to pass the time than to be productive. A validated measure of travel subjective well-being is also a significant and positive factor, suggesting people are more likely to choose a mode that makes them happier. Overall, PUT measures greatly increase the explanatory power of the mode choice model. These findings make significant contributions to travel behavior research methods and knowledge. They also offer important implications for transportation policies around promoting nonautomobile travel and planning for autonomous vehicles. 


\section{Acknowledgments}

This research was supported by a Doctoral Dissertation Fellowship from the National Institute for Transportation and Communities (NITC), a program of the Transportation Research and Education Center (TREC) at Portland State University (PSU). Financial support for my doctoral studies and research was also made possible by several graduate fellowships from the Dwight David Eisenhower Transportation Fellowship Program, part of the Office of Innovative Program Delivery (OIPD) at the Federal Highway Administration (FHWA).

Thanks are due to the many individuals who assisted in the data collection effort. From the dozens of people who provided feedback on earlier versions of the questionnaire to the hundreds of people who spent upwards of an hour of their time answering detailed questions about a recent commute trip, these respondents' participation fundamentally made this research possible. Staff at the Portland region's transportation management associations and other organizations greatly assisted in the administration of this survey by forwarding email invitations, posting information in newsletters and on websites, and otherwise helping to reach the target audience of commuting workers. Special thanks go to Scott Cohen of the Portland Bureau of Transportation for providing access to the SmartTrips email list, which alone generated hundreds of responses.

Regarding this manuscript, Susan Abe's copy editing of Chapter 5 significantly improved not only that chapter but the rest of the document as well. The literature review of Chapter 2 also greatly benefited from Patricia Mokhtarian's critical conceptual perspectives and constructive comments. In fact, her groundbreaking work on the positive 
utility of travel concept (and many other areas of travel behavior research) directly inspired this dissertation and continues to inspire its extensions.

The faculty and programs at PSU provided an excellent environment for doing dissertation research and completing a $\mathrm{PhD}$ in transportation. Foremost, I am indebted to Kelly Clifton, my advisor of six years, for teaching me how to do research, write technical papers, think critically, and otherwise survive in academia. The intellectual freedom and encouragement she provided to pursue a more theoretical topic not related to a currently funded research project was crucial. Other dissertation committee members also supplied key contributions: Liming Wang offered valuable methodological suggestions; Cynthia Mohr supported an engineering student's dabble in psychology; and Jennifer Dill always encouraged attention to the social and practical implications of technical research. Other current and former Portland State faculty also made this a wonderful place to go to school. Notably, Jason Newsom's series of courses on data analysis methods—including structural equation modeling - were among the best I have ever taken. His clear, concise, yet intuitive expositions of technical statistical concepts offer a goal to which to aspire.

Fellow PSU transportation students contributed substantially to the worth and pleasure of a PhD. The camaraderie I experienced in classes and as a member of Students in Transportation Engineering and Planning (STEP) was a valuable part of early graduate school. More recently, the convening of the $\mathrm{PhT}$ ( $\mathrm{PhD}$ Students in Transportation) group for drinks and discussion, including intellectually stimulating conversations with Joe Broach and others, provided a frequent and necessary boost of energy. My cubicle colleagues, including Kristi Currans, Alex Bigazzi, and Jaime Orrego, were always there to offer their expertise, advice, and daily cheer. In particular, Steve Gehrke has been a 
strong source of support and friendship ever since we first met while both visiting PSU in 2011. I am grateful to him for always being there to discuss ideas, to help solve analysis problems, to share advice on other aspects of work and of life, and to navigate our way along the challenging $\mathrm{PhD}$ trail together.

Finally, this dissertation was only possible because of the constant support of friends and family everywhere but especially in Portland. Watching friends from undergrad succeed in their $\mathrm{PhD}$ endeavors provided the motivation to persevere through my own. My parents and relatives not only shared their love, encouragement, and many home-cooked meals, but also helped me practice explaining technical concepts to a lay audience. Last, but most, I thank my wife Jen Kirk for her strong and unwavering love, support, and enthusiasm. It was during our "staycation" at McMenamins Grand Lodge in November 2014 that I first settled on studying the positive utility of travel concept. Without her toleration of long days and weekends hiding in front of the computer, encouragement throughout, and motivation at the end for getting it done, this dissertation (and indeed my $\mathrm{PhD}$ ) would not have been possible. 


\section{Table of Contents}

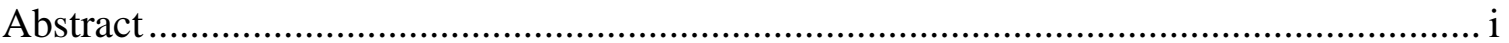

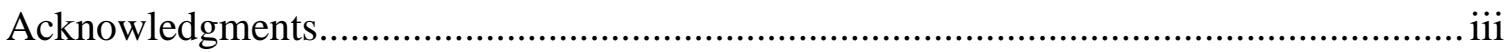

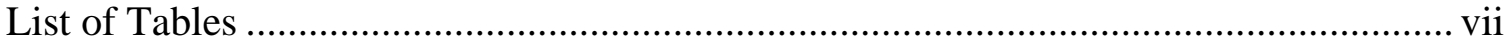

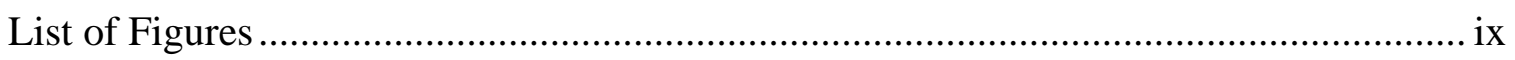

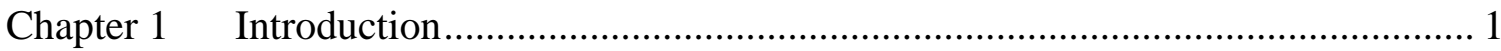

Chapter 2 Literature review ......................................................................... 14

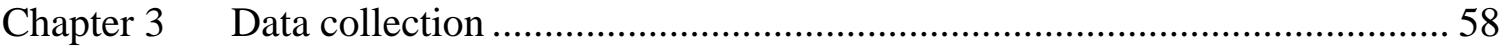

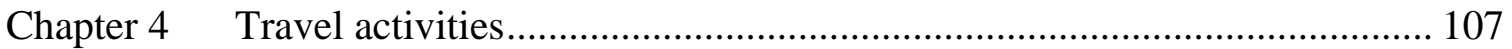

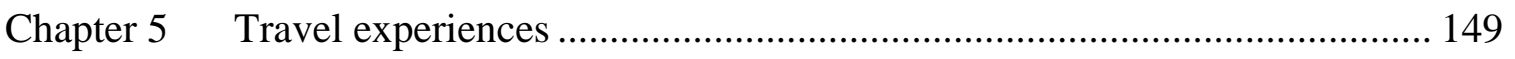

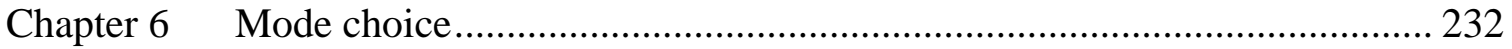

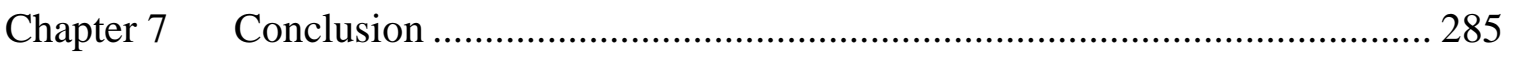

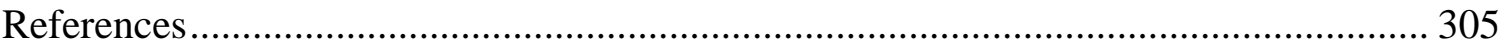

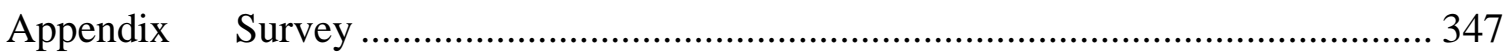




\section{List of Tables}

Table 2.1 Summary of the components of the positive utility of travel concept ........ 23

Table 2.2 Types of measurement methods and evidence of a PUT .......................... 30

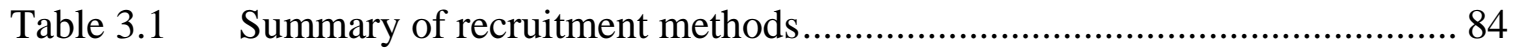

Table 3.2 Number of respondents completing each section of each survey .............. 90

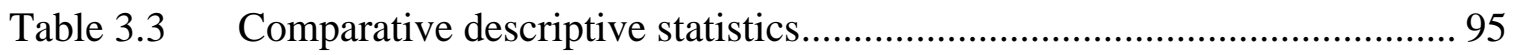

Table 4.1 Results of exploratory factor analysis of activity participation ............... 119

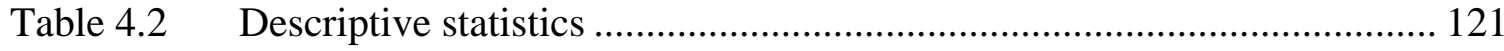

Table 4.3 Summary statistics of binary logit models of activity participation ......... 126

Table 4.4 Results of binary logit models of activity participation......................... 127

Table 4.5 Results of ordered logit models of travel usefulness ............................. 133

Table 5.1 Studies measuring the Satisfaction with Travel Scale or its variants ....... 160

Table 5.2 Items included in the Satisfaction with Travel Scale ............................. 170

Table 5.3 Questions/items used to measure travel affect................................... 172

Table 5.4 Questions/items used to measure travel eudaimonia ............................ 174

Table 5.5 Goodness-of-fit statistics for CFAs of commute well-being ................... 177

Table 5.6 Correlations between items on the STS ............................................ 178

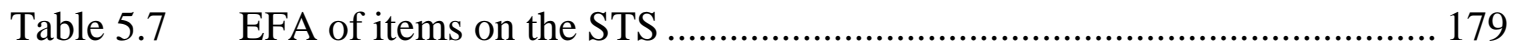

Table 5.8 Correlations between items measuring travel affect ............................. 183

Table 5.9 EFA of items measuring travel affect .............................................. 184

Table 5.10 Correlations between items measuring travel eudaimonia ...................... 188

Table 5.11 EFA of items measuring travel eudaimonia....................................... 189

Table 5.12 MIMIC model results for the STS .................................................... 195 
Table 5.13 MIMIC model results for travel affect............................................. 201

Table 5.14 MIMIC model results for travel eudaimonia ....................................... 208

Table 5.15 Correlations between factor scores for travel SWB constructs ............... 216

Table 6.1 Goodness-of-fit statistics for ICLV models of mode choice ................... 258

Table 6.2 DCA results for model with level-of-service attributes and trip and traveler

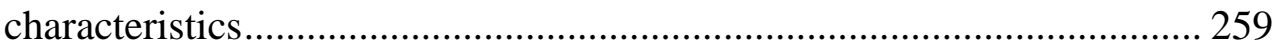

Table 6.3 ICLV results for model with PUT measures of travel activities (participation)

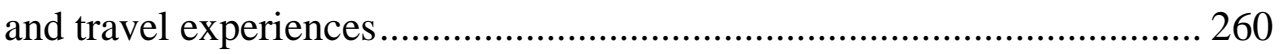

Table 6.4 ICLV results for model with PUT measures of travel activities (duration) and

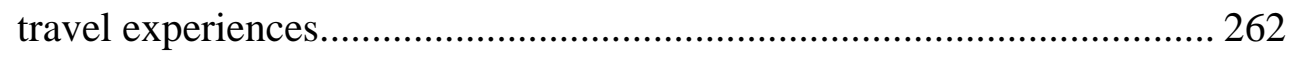




\section{List of Figures}

Figure 1.1 Conceptual framework for exploring the PUT concept and mode choice.... 7

Figure 2.1 Convincingness and specificity of types of PUT methods and evidence ... 32

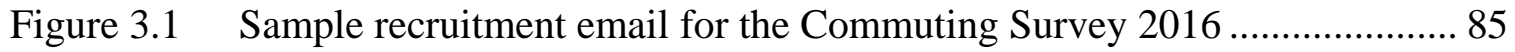

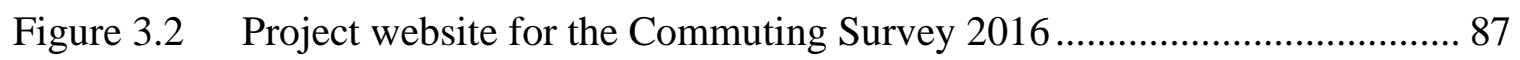

Figure 3.3 Box-and-whisker plots of survey completion times ............................. 92

Figure 3.4 Frequency of survey responses by date ........................................... 93

Figure 3.5 Home (upper) and work (lower) locations for survey respondents ........... 98

Figure 3.6 Frequently reported activities overall ............................................ 100

Figure 3.7 Travel usefulness by commute mode.............................................. 101

Figure 3.8 Frequently reported travel affect items overall .................................. 102

Figure 3.9 Frequently reported travel eudaimonia items overall ........................... 104

Figure 3.10 Box-and-whisker plot of averaged STS scores by commute mode .......... 105

Figure 3.11 Travel liking by commute mode ..................................................... 106

Figure 4.1 Frequently reported activities by commute mode............................... 125

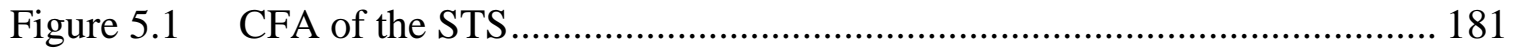

Figure 5.2 CFA of a measurement model of travel affect................................... 186

Figure 5.3 CFA of a measurement model of travel eudaimonia .............................. 191

Figure 5.4 Box plots of STS factor scores by commute mode ............................... 193

Figure 5.5 Frequently reported travel affect items by commute mode .................... 198

Figure 5.6 Box plots of travel affect factor scores by commute mode .................... 200

Figure 5.7 Frequently reported travel eudaimonia items by commute mode............ 205

Figure 5.8 Box plots of travel eudaimonia factor scores by commute mode ............. 206 


\section{Chapter 1 Introduction}

\subsection{Motivations}

Why do people travel? This fundamental question underlies most research in the travel behavior field. Many studies, methods, and models-especially those based in the derived-demand paradigm of travel analysis - implicitly or explicitly assume that people travel to reach destinations where they can conduct activities. Thus, travel is done as a means to an end; travel demand is derived from the demand for spatially separated activities; and travel time is a disutility that travelers desire to minimize. From these and other assumptions, analysts can derive willingness-to-pay measures such as the value of travel time savings (VTTS): the amount people would be willing to spend to reduce their travel time at the margin. Microeconomic theory says that these values must be positive (Hess, Bierlaire, \& Polak, 2005); that is, people would never like traveling so much that they would require payment in order to reduce travel amounts. These assumptions about travel as a derived demand also underlie the proposed advantages of the activity-based approach to travel demand modeling and forecasting (Kitamura, 1988; Pas, 1985): Knowing more about what activities people must and want to do on a daily basis greatly improves our understanding of transportation patterns and travel behaviors.

While most people and most instances of personal transportation may indeed be driven (pardon the pun) by a desire or need to do something somewhere else, these assumptions may not be universally true. Indeed, there may be other intrinsic motivations for traveling (Mokhtarian, Salomon, \& Singer, 2015). Some commuters may choose to use certain modes in order to multitask and make productive use of their travel time: e.g., by 
working or sleeping on the train, listening to music or the news while driving, or exercising while bicycling to work instead of going to the gym. Other travelers may modify their behavior or make new trips for reasons related to positive aspects of the travel experience: e.g., taking a longer route to see pleasant scenery; going for a walk to enjoy the fresh air; driving a sports car to be "seen" or feel powerful; riding public transit for environmental reasons; or using the commute as an escape, buffer, or transition between home and work. These benefits to traveling - making use of travel time through travel-based multitasking, enjoying aspects of the experience of traveling itself-are unaccounted for in VTTS estimates and in most travel behavior and mode choice studies.

The ideas that travel can provide benefits and may be motivated by factors beyond reaching activity destinations have been assembled into a concept known as "the positive utility of travel" (PUT). Based on earlier work but popularized by Salomon and Mokhtarian (1998; Mokhtarian \& Salomon, 2001; Mokhtarian, 2005), the PUT concept has spawned a small but growing area of research within the greater travel behavior arena. These studies focus on the latter two aspects of Mokhtarian and Salomon's "tripartite nature of the affinity for travel" (2001, p. 701): "activities that can be conducted while traveling" (travel activities) and "the activity of traveling itself" (travel experiences). The PUT notion assembles several concepts relevant to travel behavior: utility maximization (McFadden, 2001a), motivation theory (Ryan \& Deci, 2000), and multitasking (Kenyon, 2010), among others. Many of the intrinsic motivations related to the travel experience are founded in psychological conceptualizations of subjective well-being (SWB), including both hedonic and eudaimonic aspects: positive emotions and feelings of pleasure or happiness; and 
finding purpose, meaning, and self-actualization (De Vos, Schwanen, Van Acker, \& Witlox, 2013; Ryan \& Deci, 2001).

Despite (or perhaps because of) these varied theoretical backgrounds, empirical analyses of the PUT concept remain limited in both quantity and scope. Fundamentally, there is a need for further theoretical development and classification of the PUT idea and its multifaceted aspects. An added challenge is the way in which researchers conceive of PUT differently, use varying language when describing these concepts, and ask an assortment of questions that may or may not provide convincing evidence. Understanding clearly what is and is not an instance of a PUT, and highlighting successful ways of measuring PUT attributes, would be useful for future research. Additionally, few empirical studies investigate both major aspects of the PUT concept-travel activities and travel experiences-simultaneously, focusing instead on either one or the other. With the exception of the Satisfaction with Travel Scale (STS) (Ettema et al., 2011), most existing psychological instruments for measuring SWB have rarely been tested in the travel domain. Finally, research is only just beginning to empirically examine the potential for PUT aspects to affect travel behaviors like mode choice (e.g., Malokin, Circella, \& Mokhtarian, 2015). Many important tasks in the conceptualization, measurement, and modeling of the PUT concept remain incomplete.

Because of these gaps, improvements to our collective knowledge of and ability to analyze PUT-related effects on travel behavior remain unrealized. The lack of evidence regarding the existence and magnitude of (for instance) mode choice impacts of the PUT concept's travel activity and experiential aspects limits the ability of engineers, planners, and policymakers to anticipate and plan for future transportation needs. If travel-based 
multitasking and expectations of positive emotions or fulfillment affect the willingness of people to pay for travel time savings, empirically-derived VTTS estimates may be biased due to endogeneity (Fernández-Antolín, Guevara, de Lapparent, \& Bierlaire, 2016; Singleton \& Clifton, 2015), affecting appraisals and cost-benefit analyses of multimillion/billion-dollar mobility-enhancing transportation projects. Lacking guidance for expected impacts of policy interventions that rely on PUT-related aspects-such as those designed to promote nonautomobile modes through strategies like protected bike lanes and social and encouragement initiatives — decisionmakers may be more reluctant to take the risk. Looking towards the future, the PUT concept is likely only to grow in importance. The rapid development of semi- and fully-autonomous vehicles (AVs) portends major disruptions in mobility patterns and transportation planning orthodoxy on the horizon. AVs' likely abilities to facilitate substantial increases in travel-based multitasking while simultaneously making travel less stressful and more enjoyable are at the heart of their attractiveness. Understanding travel behavioral impacts of and sensitivities to PUT attributes today could help to anticipate the potential impacts of AVs tomorrow.

\subsection{Research questions}

This dissertation aims to address some of the theoretical, measurement, and empirical gaps and limitations mentioned above, thus offering improved transportation policy guidance. Specifically, it investigates answers to the following research questions: 


\section{What is the positive utility of travel (PUT) concept?}

a. Conceptually, how is the PUT idea defined and structured?

More rigor and structure is required in the conceptualization of the PUT notion. Is there a clear but comprehensive definition for what is a PUT? What components comprise the PUT concept? How can we theoretically distinguish between them? How is the PUT concept related to other topics, such as SWB and VTTS?

b. Empirically, how can a positive utility of travel be measured?

Researchers would benefit from a closer and theoretically informed investigation into methods for measuring the PUT concept, including both travel activity and travel experience aspects. Can these two aspects be empirically distinguished? What survey questions and items best illuminate them?

\section{What are potential determinants of a PUT?}

What personal and travel characteristics predict PUT measures?

Empirical research has begun to examine factors associated with measures of the PUT concept, but more evidence would be useful, particularly using theoretically valid metrics. How do PUT attributes vary across modes? Are some modes more conducive to travel-based multitasking than others? Are ratings of SWB higher for certain modes than for others? Are other trip characteristics significantly associated with PUT? What personal (socio-demographic and perceptual) attributes significantly predict PUT measures? 


\section{How do positive utilities of travel affect travel behavior?}

How do PUT attributes impact travel mode choice?

Few studies investigate the potential effects of PUT attributes on travel behaviors like mode choice, and almost none have considered both travel activity and travel experience aspects of the PUT concept. Thus, there is little guidance as to the existence and magnitude of this potential relationship. The premise of this dissertation is that PUT factors likely do affect mode choice behavior at least modestly, yet this remains an open question subject to empirical examination. Are measures of PUT significantly associated with mode choice? In what directions? How strongly do PUT attributes affect mode choice, compared to traditional level-of-service (travel time and cost) factors and sociodemographic traveler characteristics? Do estimated parameters and sensitivities offer transportation policy implications?

\subsection{Approach}

These research questions are addressed through a comprehensive theoretical, empirical, and analytical approach involving novel primary data collection and cutting edge modeling methods. The conceptual diagram shown in Figure 1.1 reflects this approach and guides the empirical analyses. 


\section{Traveler characteristics}

\section{Socio-demographics}

age, gender, race/ethnicity, education, disability, household size, income, vehicle availablity,

housing \& employment characteristics

\section{PUT perceptions}

travel time satisfaction, ideal travel time, teleportation test

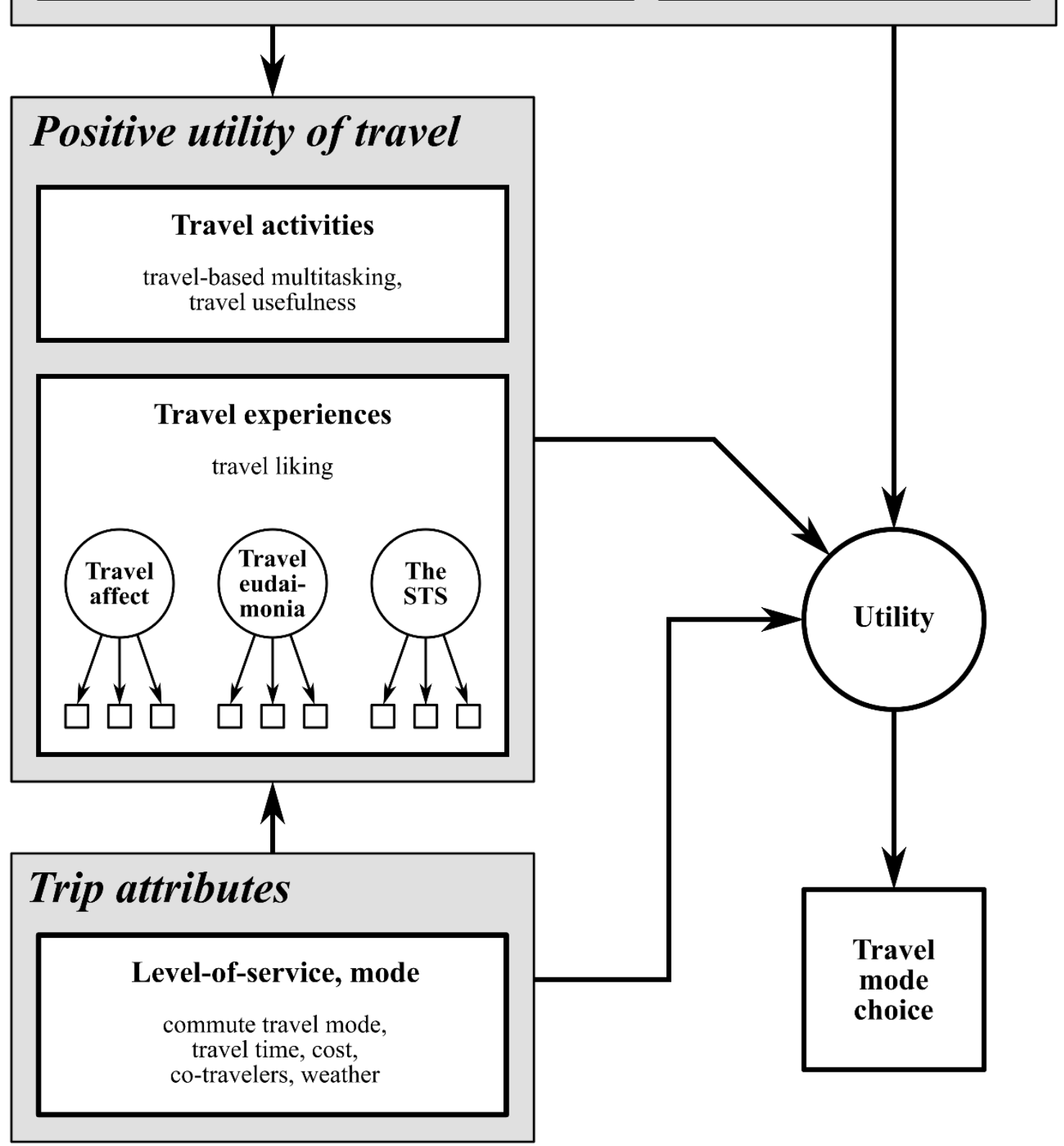

Figure 1.1 Conceptual framework for exploring the PUT concept and mode choice 
The first step in this process is to address conceptual questions related to the PUT concept (RQ1a). To do this, a literature review is conducted that assembles theoretical and empirical studies with relevance for understanding the PUT concept. The review presents a detailed conceptualization of PUT and links it to concepts like utility, motivation, and SWB. It also distinguishes between two key PUT aspects: those related to travel activities (like travel-based multitasking), and those related to travel experiences (like SWB). Next, various measurement methods and types of evidence for a PUT are examined, categorized, and critiqued. Lastly, the more limited evidence surrounding determinants of a PUT and the impact of PUT measures on travel behavior are summarized and discussed.

Next, a survey is designed to collect original data on the PUT concept and individual travel behaviors in a way that can illuminate the PUT relationship with mode choice for a common trip purpose: commuting to work. Based on the results of the literature review, the questionnaire uses best practice trip-based PUT measures when they exist and creates new ones when they do not or are inadequate. In a substantial improvement over previous studies, this survey measures attributes related to both travel activity and travel experience aspects. Uniquely, it also solicits responses to these questions not just for the mode used on the most recent commute trip but also for other modes that were considered, eliminating the need to model or make assumptions about this information.

The data analytic approach, illustrated in Figure 1.1, involves several steps. First, the specific measures of the PUT concept are examined using a variety of analytical techniques. In the case of travel activities, this involves the use of exploratory factor analysis (EFA) to identify groups of activities commonly engaged in together. For travel experience aspects, both exploratory and confirmatory factor analysis (CFA) are conducted 
to identify and/or confirm the existence of common unobserved constructs related to SWB, thus developing measurement models of travel affect, travel eudaimonia, and the STS. These analyses, depicted in the central PUT box of Figure 1.1, address the empirical question about how best to measure the PUT concept (RQ1b).

Following the empirical examination of PUT measures, the next step involves investigating their potential determinants. These explanatory factors include information about travelers - socio-demographic characteristics and perceptual attributes - as well as trip-related information like commute mode and travel time. First, variations in PUT aspects across modes are examined qualitatively and visually through simple modal segmentations. Next, models are estimated predicting the PUT measures as a function of trip and traveler characteristics. For travel activities, this involves binary logit models of participation in different types or groups of activities, and ordered logit models of a single measure of travel usefulness. For travel experiences, this involves structural equation modeling (SEM) in the form of multiple indicators, multiple causes (MIMIC) models, in which exogenous variables predict latent PUT variables from the CFA models. These analyses address the research question regarding determinants of a PUT (RQ2) and are represented by the vertical arrows leading into the PUT box from Traveler characteristics and Trip attributes.

Finally, the last step in this process addresses the research question regarding the relationship between PUT attributes and commute mode choice (RQ3). This step involves enhancing a traditional mode choice model — using level-of-service measures (travel time and cost) that vary across alternatives and traveler characteristics (socio-demographics) that do not-with measures of the PUT concept. Because the travel experience components 
are expressed as unobserved constructs from the CFA/SEM analysis, this approach adopts a state of the art technique called integrated choice and latent variable (ICLV) modeling (also known as hybrid choice modeling). While complex to specify and estimate, ICLV models simply allow unobserved latent variables to enter a discrete choice model's utility equation as predictors. This modeling effort produces estimates of the association between PUT measures and commute mode choice behavior, offering rare empirical evidence into the relationships between the two.

\subsection{Overview}

This dissertation is organized into several chapters that roughly reflect the different stages of the research approach described in the previous section. This introduction section comprises Chapter 1 . The remaining chapters are summarized in the following paragraphs.

Chapter 2 presents a literature review of the PUT concept. In it, a PUT is defined simply as "any benefit(s) accruing to a traveler through the act of traveling." The major components of the PUT concept-destination activities, travel activities, and travel experiences - are also distinguished. Next, methods for measuring a PUT and corresponding empirical evidence are critiqued and reviewed, suggesting that direct questions about travel-based multitasking and positive emotions or satisfaction from the travel experience offer the most convincing evidence. Results regarding potential determinants of these PUT aspects are also summarized. Finally, the limited number of studies examining associations between PUT attributes and travel behaviors like mode choice are reviewed, concluding that more research is necessary before we can begin to understand these relationships more clearly. 
Chapter 3 documents the development and administration of an original data collection effort to measure the PUT concept and its relationship with commute mode choice. The Commuting Survey 2016 was administered to commuters in the Portland, OR, metropolitan area during the fall of 2016. About 650 complete responses were received to the main Part I of the survey; around 475 people completed an optional Part II of the survey. This chapter discusses the development of specific questions and items related to travelbased multitasking and subjective well-being, the recruitment of participants through their place of employment (enhanced with some direct emailing and field recruitment), and efforts involved in data processing. It also includes a detailed description of the online questionnaire and its two parts; the full survey instrument is attached in the Appendix.

Chapter 4 deals with measuring and analyzing the potential determinants of the travel activity aspects of the PUT concept: self-reports of travel-based multitasking and a single question about travel usefulness. It describes the estimation of several binary logit models to predict engagement in multiple activities while traveling, including two groups of activities found from the EFA. The results from an ordered logit model predicting travel usefulness as a function of trip and traveler characteristics are also presented. This chapter concludes with a discussion of key findings: Nonmotorized commuters appeared to have more useful commutes because of an ability to exercise; perceptions seemed to play a bigger role than socio-demographic characteristics; and many travelers may be doing things just to pass the time rather than making productive use of their travel time.

Chapter 5 describes the analysis of three key measures of the travel experience aspects of the PUT concept: the Satisfaction with Travel Scale (STS), travel affect, and travel eudaimonia. First, these concepts are reviewed, and measurement limitations are 
discussed. Next, EFAs and CFAs are presented that confirm a three-factor structure of the STS and develop new four-factor measurement models of travel affect ("Distress," "Fear," "Attentiveness," and "Enjoyment") and travel eudaimonia ("Security," "Autonomy," “Confidence," and "Health"). Significant modal variations — walking and bicycling modes rated higher on many constructs, including the STS — are also discussed. Then, results of MIMIC models predicting travel experience PUT latent variables as a function of trip and traveler characteristics are presented. Finally, key findings are discussed: distinctions between operating and nonoperating and between more and less physically active modes; women reporting lower levels of SWB; and the relative weakness in the explanatory power of objective traveler characteristics.

Chapter 6 covers the specification, estimation, and analysis of an ICLV mode choice model including PUT measures. It describes how the mode choice model adds measures of travel-based multitasking, travel usefulness, the STS, and travel liking (presented in previous chapters) to traditional trip and traveler characteristics in order to understand factors influencing around 550 observations of commute mode choice behavior. The chapter also describes the unique nature of this dataset: It used realistic choice sets and measured PUT attributes for chosen and alternative modes. It concludes with a discussion of results documenting a link between the PUT concept and mode choice: significant associations with mode choice for activity participation and the STS-based measure of SWB.

Chapter 7 concludes the dissertation with a summary of the motivations for this research and its key findings, along with a retrospective assessment of answers to the research questions. It then highlights the key contributions of this research, making strides 
towards better conceptualization, measurement, and evidence for the PUT concept and its association with mode choice behavior. Finally, the chapter concludes with a discussion of research and policy implications (including towards an understanding of the potential impacts of autonomous vehicles), as well as limitations of the study and opportunities for future work. 


\section{Chapter 2 Literature review}

\section{Reviewing concepts, measures, and evidence of the positive utility of travel concept}

\subsection{Abstract}

Most work in the transportation field assumes traveling is a means to an end, travel demand is derived (from activity demand), and travel time is a disutility to be minimized. In contrast, the positive utility of travel (PUT) concept suggests that travel can provide benefits and may be motivated by factors beyond reaching activity destinations. This literature review first presents a detailed conceptualization of the PUT notion and links it to concepts like utility, motivation, and subjective well-being. The major components of PUT are destination activities, travel activities (multitasking), and travel experiences; this study focuses on the latter two of these three. Next, various measurement methods and types of evidence are considered, categorized, and evaluated for their convincingness. The most useful methods appear to involve direct questioning or assessments of travel-based multitasking and positive affect or satisfaction from the travel experience. Then, evidence for determinants of PUT and its effects on travel behavior are summarized; both topics (especially the latter) are understudied and present opportunities for future research. This review concludes with a discussion of the PUT concept's implications for transportation research, planning, policy, and the future. The impact on behavior of a positive utility of travel will only grow in importance with looming technological changes. 


\subsection{Introduction}

Why did the pedestrian cross the road? "To get to the other side, obviously," says the transportation paradigm in which traveling is a means to an end, travel demand is derived (from the demand for spatially separated activities), and travel time is a disutility to be minimized. Indeed, maybe our pedestrian was catching her bus to work or going to the grocery store. Perhaps walking was the quickest or cheapest mode, and the crossing was the safest or most direct. Now, consider possible alternative answers to our initial question. Maybe our pedestrian was strolling through her neighborhood for exercise or to clear her head. Perhaps she chose not to drive so she could instead talk on the phone, enjoy the outdoors, or express a proenvironmental attitude. In these cases, traveling was less about minimizing disutility and more about maximizing pleasure or happiness.

The idea that travel can provide benefits and may be motivated by factors beyond reaching activity destinations is known in the travel behavior field as "the positive utility of travel" (PUT) concept. The examples above show that travel time can be used productively and that travel can provide physical, emotional, and symbolic benefits. The PUT idea brings together a number of concepts relevant to travel behavior: utility maximization, motivation theory, satisfaction and subjective well-being, and multitasking, among others. It also has important implications for transportation research, planning, policy, and practice. Positive utilities of travel may affect economic willingness-to-pay measures that are crucial to the assessment of transportation projects. A more complete understanding of the PUT concept might improve forecasts of walking, bicycling, and transit demand or help to design interventions to increase the use of these active 
transportation modes. In the future, increases in productivity made possible by autonomous vehicles have the potential to dramatically change how people get around.

This review's (potentially ambitious) objective is to present a deeper understanding of the positive utility of travel idea and associated travel behavior phenomena. Since the PUT concept was coined (Salomon \& Mokhtarian, 1998; Mokhtarian \& Salomon, 2001) and gained broader attention (Mokhtarian, 2005) in the travel behavior research arena, many studies have examined various PUT aspects, both empirically (e.g., Ory \& Mokhtarian, 2005; Diana, 2008; Malokin et al., 2015) and more conceptually (e.g., Hess et al., 2005; Mokhtarian, Salomon, \& Singer, 2015; Singleton \& Clifton, 2015). Despite this small but growing body of work, no single source comprehensively reviews methods of analysis and empirical evidence related to the PUT concept. Through a careful reading, synthesis, and critique of existing theoretical and empirical literatures ${ }^{1}$, this review aims to advance research in this area. As a result, its intended audience includes travel behavior researchers, who may be interested in working in this domain, as well as transportation practitioners, who may want to apply research findings.

To achieve this objective, this review's goals are three-fold. The first section tackles a fundamental question: What is the positive utility of travel (PUT) concept? Drawing upon economic and psychological perspectives, it conceptually details and expands upon Mokhtarian and Salomon's (2001) "tripartite nature of the affinity for travel." The next

\footnotetext{
${ }^{1}$ Literature was selected using a mix of methodologies. First, a few seminal papers (Hess et al., 2005; Lyons \& Urry, 2005; Mokhtarian \& Salomon, 2001; Mokhtarian, Salomon, \& Singer, 2015; Salomon \& Mokhtarian, 1998) were identified using personal knowledge. Next, both backward and forward snowballing were used to gather additional papers, which were then reviewed and categorized. Finally, Google Scholar and TRID databases were queried using basic search terms identified in the previous categorization to retrieve additional sources. For the most part, only articles published in transportation-related journals (or recent unpublished works) were considered.
} 
section addresses a practical issue: How can a positive utility of travel be measured? To answer this question, various measurement methods and types of evidence of a PUT are examined and categorized according to their roles illuminating different PUT aspects and their convincingness. As part of this, related empirical questions are briefly investigated: What are determinants of a positive utility of travel? How do positive utilities of travel affect travel behavior? Answers to each of these questions could be the subject of a separate review. Together, these sections highlight empirical research gaps to be filled. Finally, this review concludes by offering potential implications of and guidance for future research on the PUT concept.

\subsection{What is the Positive Utility of Travel (PUT) concept?}

\subsubsection{Utility}

Before discussing the PUT concept itself, it is instructive to step back and clarify the definition of "utility." Utility has historically played a starring role in the fields of economics and psychology. Philosopher Jeremy Bentham (1789/1948) described utility as

pleasure (positive) over pain (negative). In the more modern economic sense, utility measures the value of a preference-satisficing decision, meant to represent personal satisfaction from consuming a good or service. Microeconomic theories of rational consumer behavior - a foundation of discrete choice models used in travel behavior analysis (Ben-Akiva \& Lerman, 1985) - presume that an individual, when faced with a decision, has consistent and transitive preferences that can be expressed as an ordinal utility function, and that the individual makes a consumption decision or choice to maximize 
her/his utility, subject to constraints. Many models statistically infer this decision utility from observed decisions and behaviors, presuming imperfect and inaccurate knowledge of the true decision-making processes.

In contrast, psychological perspectives of utility are closer to Bentham's original definition. In that field, satisfaction or well-being is frequently self-reported using individuals' answers to questions (often with Likert-type scales). This introduces a temporal issue: Depending on when questions are asked with respect to the consumption activity or decision, the measured utility could be anticipated, experienced, or remembered. These self-reported utilities naturally differ from one another (Kahneman, Wakker, \& Sarin, 1997), from inferred decision utility, and even potentially from the latent underlying utility they are trying to measure. The differences between economic and psychological approaches to utility measurement raise important moral and empirical questions that are beyond the scope of this review ${ }^{2}$. More broadly, utility's etymology (from the Latin "utilis," meaning useful) and association with utilitarian or practical benefits has led to the criticism (Nordbakke \& Schwanen, 2014) that utility-based approaches are too narrowly focused on hedonism (pleasure, happiness, and the satisfaction of desires) at the expense of eudaimonia (purpose, goal-attainment, and self-realization). Many perspectives of wellbeing (De Vos et al., 2013; Ryan \& Deci, 2001; Waterman, 1993) include both hedonic and eudaimonic components (see the later section on Subjective well-being).

For the purposes of this review, "utility" is considered to be an imperfect name for a broad concept encompassing aspects of pleasure, preference satisfaction, and (hedonic

\footnotetext{
2 These temporal and crossdisciplinary issues with utility measurement have been occasionally considered in the transportation literature (Ettema et al., 2010; Abou-Zeid \& Ben-Akiva, 2012; De Vos et al., 2016).
} 
and eudaimonic) well-being. Also, both economic (inference) and psychological (selfreporting) approaches may be valid ways - particularly in conjunction - to measure this utility concept.

\subsubsection{Motivation}

Illuminating the positive utility of travel also requires exploring motivations for personal transportation. Psychology has long focused on trying to understand the motivations underlying human behavior. One perspective is Maslow's (1943, 1954) hierarchy of five needs motivating human actions, occasionally mentioned in travel behavior literature (Alfonzo, 2005; Dal Fiore, Mokhtarian, Salomon, \& Singer, 2014; Mokhtarian, Salomon, \& Singer, 2015; Rasouli \& Timmermans, 2014c; Salomon, 1985; Singleton, 2013). The most basic needs are physiological (homeostasis, maintaining the body) and protective/safety (avoiding illnesses and threats). Next are two social needs: love, affection, and belongingness; and esteem, appreciation, social status, or selfconfidence. The final need is self-actualization (fulfilling one's potential).

Another useful distinction is between extrinsic and intrinsic motivation (Ryan \& Deci, 2000). Extrinsic (or instrumental) motives are external to the activity, which is done as a means to an end: to achieve a separate outcome such as obtaining a reward or avoiding a punishment. Intrinsic (or autotelic) motives are internal to the activity, which is done for its own sake: because of interest in or enjoyment of the activity.

The derived demand paradigm views travel as completely extrinsically motivated: to conduct other activities that fulfill many of Maslow's needs. People go to work, school, and other places to earn money, learn, eat, socialize, be entertained, etc. Certainly, travel 
for mandatory, maintenance, and even discretionary purposes (Reichman, 1976) encompass many of the lower-order needs of homeostasis, safety/security, and social belongingness; travel to access recreational activities often helps fulfill higher-order needs (Salomon, 1985). However, travel can have intrinsic motivations too: as a transition time between activities, or as a means of viewing scenery, getting exercise, feeling independent, and expressing social status (Mokhtarian \& Salomon, 2001; Salomon \& Mokhtarian, 1998). Even Maslow (1943) acknowledged that behaviors (like travel) could fulfill multiple needs simultaneously. For a much more detailed and thorough examination of the reasons for traveling, see the recent review article by Mokhtarian, Salomon, and Singer (2015).

\subsubsection{Positive utility of travel}

The positive utility of travel is not a new idea, nor is it relegated to purely academic study. Scholars, poets, philosophers, authors, and the media have discussed intrinsic rewards to traveling and moving for hundreds of years. A practical activity, walking has been elevated as a means of exploring a place, an invitation to lose oneself in thought, and a convergence of "the mind, the body, and the world" (Solnit, 2001, p. 5). It has also been imbued with symbolic and cultural significance: e.g., the Parisian figure of the flâneur and the act of flânerie, enjoying strolls through a crowded urban experience "with no destination in mind" (Shaya, 2004, p. 46). The intrinsic benefits of traveling are not reserved for human-powered motion alone. Much has been written about America's “love affair with the automobile" and its association with achievement, status, and independence (e.g., Sachs, 1992), a story that feels true, even if it was initially a mass media narrative 
fabricated by "motordom" (Norton, 2015). Mobility, or the freedom of movement, has also been described as a fundamental value and basic human right (Houseman, 1979; United Nations, 1948).

Some transportation scholars have recognized and written about PUT-related concepts. Early theoretical contributions to microeconomic time allocation theory and travel time valuation recognized that, in some (albeit few) cases, travel may be enjoyable (Becker, 1965; Evans, 1972; Johnson, 1966). Notably, Oort (1969) mentioned that pleasant or productive uses of travel time could reduce its disutility, and considered the possibility that "those who travel for travel's sake" (p. 283) may have a positive utility associated with travel time. Additional conceptual progress occurred in the late 1970s and early 1980s. Reichman (1976) questioned the notion of travel as a disutility, wondering "is transportation only a means to an end, or does it really fulfill some ends in itself' (p. 148). Hupkes (1982) distinguished between travel's "derived utility" (from activities becoming possible) and "intrinsic utility" (from the satisfaction of traveling). There were likely other early mentions of PUT phenomena that have been unintentionally excluded.

One of the clearest and most influential articulations of PUT comes from Salomon and Mokhtarian (1998). They proposed that "human beings have an intrinsic drive for mobility" (p. 130), and later described the "tripartite nature of the affinity for travel" as composed of "the activities conducted at the destination," "activities that can be conducted while traveling," and "the activity of traveling itself" (Mokhtarian \& Salomon, 2001, p. 701).

Borrowing from the solid foundations of Mokhtarian, Salomon, and others, and inspired by the preceding discussions of utility and motivation, this review offers the 
following simple definition of a positive utility of travel (PUT), as any benefit(s) accruing

to a traveler through the act of traveling ${ }^{3}$. It also propose separating the PUT concept into

three components, based on different sources of benefits:

1. Destination activities: Benefits from reaching a destination with activity potential;

2. Travel activities: Benefits from using travel time for activity participation (travel-

based multitasking); and

3. Travel experiences: Benefits from the experience of traveling, including from

a. Affective (hedonic) enjoyment of the travel experience, and

b. Symbolic (eudaimonic) expressions or fulfillment from the travel

experience.

These components of PUT are summarized in Table 2.1 and detailed in the following

sections.

3 A few notes about this definition of the PUT concept are warranted. First, benefits may come in many
forms, including: direct benefits to the traveler via more positive affect, increased health and well-being,
greater productivity, monetary gain, or sensations of eudaimonia; and indirect benefits to other people the
traveler interacts with and society at large. Second, since the focus of many studies is on understanding and
explaining individuals' behaviors, researchers are primarily interested in benefits that influence (conscious
or unconscious) decision-making processes. These direct and ancillary benefits influence behavior only if the
traveler values them or if she/he can acquire or transact them. For instance, travel can be a time of transition
(Jain \& Lyons, 2008), allowing an employee to prepare for a presentation (benefitting the employer and
coworkers); these benefits might accrue to the traveler only indirectly, through anticipation of an improved
working environment or career advancement. As another example, a traveler could capture some of the
external benefits of bicycling over driving (e.g., reduced congestion, air pollution, and greenhouse gas
emissions) if there were some sort of "commuter carbon market" for selling her/his carbon offsets. In an
individual example, a traveler will likely gain some long-term health benefits of physical activity whenever
she/he walks or cycles. However, the degree to which this benefit influences travel behaviors depends upon
how much a traveler perceives and values that exercise. Therefore, it may be more accurate to refer to the
subjective positive utility of travel. Third, the act of traveling should be viewed broadly: both as a result of
traveling (travel as the cause) and during the course of traveling (travel as the setting). 


\section{Table 2.1 Summary of the components of the positive utility of travel concept}

\begin{tabular}{|c|c|c|c|}
\hline & 1. Destination activities & 2. Travel activities & 3. Travel experiences \\
\hline $\begin{array}{l}\text { Mokhtarian \& } \\
\text { Salomon, } \\
2001\end{array}$ & $\begin{array}{l}\text { "The activities } \\
\text { conducted at the } \\
\text { destination." }\end{array}$ & $\begin{array}{l}\text { "Activities that can be } \\
\text { conducted while } \\
\text { traveling." }\end{array}$ & "The act of traveling itself." \\
\hline Definition & $\begin{array}{l}\text { Benefits from reaching a } \\
\text { destination with } \\
\text { activity potential. }\end{array}$ & $\begin{array}{l}\text { Benefits from using } \\
\text { travel time for activity } \\
\text { participation (travel- } \\
\text { based multitasking). }\end{array}$ & $\begin{array}{l}\text { Benefits from the experience of } \\
\text { traveling, including from } \\
\text { a. Affective (hedonic) } \\
\text { enjoyment of the travel } \\
\text { experience; } \\
\text { b. Symbolic (eudaimonic) } \\
\text { expressions or fulfillment } \\
\text { from the travel experience. }\end{array}$ \\
\hline Summary & Travel to go somewhere. & $\begin{array}{l}\text { Travel as the setting for } \\
\text { other activities. }\end{array}$ & $\begin{array}{l}\text { a. Travel as the setting for } \\
\text { experiences generating } \\
\text { positive emotions; } \\
\text { b. Travel as a means to a } \\
\text { fulfilling or meaningful end. }\end{array}$ \\
\hline $\begin{array}{l}\text { Motivations for } \\
\text { traveling }\end{array}$ & Extrinsic/instrumental. & $\begin{array}{l}\text { Often extrinsic or } \\
\text { instrumental, but } \\
\text { could be intrinsic or } \\
\text { autotelic. }\end{array}$ & $\begin{array}{l}\text { Partially or fully } \\
\text { intrinsic/autotelic: } \\
\text { a. Hedonic/affective; } \\
\text { b. Eudaimonic/symbolic. }\end{array}$ \\
\hline Shorthand & $\begin{array}{l}\text { Going to do something } \\
\text { in a place ("going } \\
\text { ing"). }\end{array}$ & $\begin{array}{l}\text { Doing something } \\
\text { (“__ing”). }\end{array}$ & $\begin{array}{l}\text { a. Experiencing something } \\
\text { ("feeling ”); } \\
\text { b. Expressing or fulfilling } \\
\text { something ("being_" or } \\
\text { "seeking "). }\end{array}$ \\
\hline $\begin{array}{l}\text { Functional } \\
\text { setting }\end{array}$ & $\begin{array}{l}\text { Done at destinations or, } \\
\text { more broadly, fixed } \\
\text { locations. }\end{array}$ & $\begin{array}{l}\text { Can usually be done as } \\
\text { well in a nontravel } \\
\text { setting. }\end{array}$ & $\begin{array}{l}\text { Cannot be easily divorced from } \\
\text { the travel setting. }\end{array}$ \\
\hline $\begin{array}{c}\text { Degree of } \\
\text { agency }\end{array}$ & Not relevant. & $\begin{array}{l}\text { Higher; can usually } \\
\text { choose when and how } \\
\text { long to participate. }\end{array}$ & $\begin{array}{l}\text { Lower; cannot easily control the } \\
\text { occurrence or duration of } \\
\text { experiential aspects. }\end{array}$ \\
\hline Examples & $\begin{array}{l}\text { Traditional activity } \\
\text { participation: e.g., } \\
\text { going shopping at the } \\
\text { mall by car; going to } \\
\text { school on the bus. }\end{array}$ & $\begin{array}{l}\text { Multitasking: reading a } \\
\text { newspaper while } \\
\text { riding the train; } \\
\text { talking on the phone } \\
\text { while walking; } \\
\text { bicycling to work. }\end{array}$ & $\begin{array}{l}\text { a. Feeling excited by the first } \\
\text { snowflakes while walking } \\
\text { home from school; } \\
\text { b. Being in control and seeking } \\
\text { social status from driving a } \\
\text { sports car. }\end{array}$ \\
\hline
\end{tabular}




\subsubsection{Destination activities}

Destination activities reflect the extrinsic and instrumental motivations for travel from the derived-demand paradigm and the activity-based approach to travel analysis: to conduct activities at spatially separated locations. Logic therefore suggests that the benefits to a traveler from accessing activity opportunities must outweigh the costs of getting there, a tradeoff most clearly expressed in destination choice models. There, the utility of choosing a destination decreases with a measure of the generalized cost (usually, time and money) of travel and increases with a function representing destination attractiveness. Attributes of attractive destinations vary, but measures include: population, employment, or area (Ben-Akiva \& Lerman, 1985); retail store size, products/prices, and quality (Koppelman \& Hauser, 1978); agglomerations of other destinations (Bernardin, Koppelman, \& Boyce, 2009); supportive pedestrian environments (Clifton, Singleton, Muhs, \& Schneider, 2016); and even “place happiness" (Deutsch-Burgner, Ravulaparthy, \& Goulias, 2014). Certainly the destination choice process warrants greater attention; Jones (1978) proposed viewing travel behavior as "an interactive tradeoff between the positive and negative features of both travel and destination" (p. 298). Nevertheless, because destination activity factors are traditionally considered in travel behavior research and demand analysis, they will not be the focus of the remainder of this study.

\subsubsection{Travel activities}

A second pathway to generating a PUT is through activities that can be conducted while traveling: in short, travel-based multitasking. The motivations for a travel activitylike any activity — can be extrinsic (e.g., preparing a presentation for work) or intrinsic 
(e.g., listening to music for the enjoyment of it), yet they are likely less closely related to the act of traveling as are travel experience aspects of the PUT concept. In many cases, travel can be thought of as simply the setting for activities that could take place elsewhere (e.g., reading a book on the bus vs. at home), although the degree to which a multitasked activity is distinct from traveling likely falls on a continuum (Circella, Mokhtarian, \& Poff, 2012). Context and setting also matter: Characteristics of the travel experience certainly affect the types of activities conducted and their quality.

Travel-based multitasking studies have generated many lists that classify and enumerate activity types (Circella et al., 2012; Circella, Salgado, Mokhtarian, \& Diana, 2015; Kenyon, 2006; Kenyon \& Lyons, 2007; Keseru et al., 2015; Lyons, Jain, Susilo, \& Atkins, 2013; Malokin et al., 2015; Ohmori \& Harata, 2008; Timmermans \& van der Waerden, 2008). Almost any activity that can be done in life can also be done while traveling: e.g., talking, reading, writing, listening to music, eating/drinking, taking in scenery, sleeping, and many more. Activities have been grouped by purposeworking/studying, maintenance, and leisure — and by the degree to which they command "the deliberate use of one's physical and/or mental faculties" (Circella et al., 2012, p. 83). Activities requiring little or no input from the individual are more passive; activities demanding significant investment of physical or mental resources are more active $\mathrm{e}^{4}$. The active-passive continuum is particularly relevant for examining modal differences in travel-based multitasking. Operating a vehicle (automobile or bicycle) involves a

\footnotetext{
${ }^{4}$ Certainly, this active/passive classification is a simplification, and many activities require varying levels of traveler interaction over their course. For instance, listening to music involves an initial selection (using both hand-eye coordination and a deliberative mental choice) followed by a period with little physical interaction but potentially low (if zoning or tuning out) or high (if intently focused) dedication of mental resources.
} 
significant investment of both physical (eyes, hands, feet) and mental resources, which practically and legally restricts activities to being more passive; automobile, taxi, or public transit passengers have many more potential activities at their disposal. (Of course, some people still engage in more active activities that distract from the driving task.)

A final note about travel activities: Distinguishing activity engagement from item use is recommended. Some activities and items are closely linked, both in the vernacular (e.g., texting involves using a phone) and in reality (e.g., books are mostly used for reading). However, different items can be used for similar activities (e.g., listening to music), and some objects can be used for many different activities (e.g., smartphones). Both activity engagement and item use are important factors for time use and activity/travel behavior analysis, yet they should be treated as two distinct aspects for the purposes of studying travel-based multitasking.

\subsubsection{Travel experiences}

Other pathways can generate a PUT through the act or experience of traveling itself. Most of these motivations are autotelic and intrinsic to traveling. Conceptually, travel experiences can be organized into two categories, roughly following motivational distinctions (hedonic vs. eudaimonic, affective vs. symbolic). First are aspects related to travel as the setting for hedonic experiences that generate positive affect (bodily sensations, feelings, emotions, or mood): e.g., traveling fast can be fun, thrilling, and rejuvenating. Second are instances where the travel experience is used to evoke eudaimonia or for symbolic reasons; travel is a means to a fulfilling end; e.g., driving a sports car can express power, mastery, and social status. A potential third category is what Mokhtarian and 
Salomon (2001) call this "undirected travel," where travel is completely primary (an end in and of itself) and the destination is completely ancillary or absent; e.g., hiking, kayaking, running, or racing. Because many instances of undirected travel are outdoor leisure and recreational activities, in which hedonic/eudaimonic motivations often dominate ${ }^{5}$, they are considered special cases of the first two types of travel experiences.

Many authors have investigated how travel can evoke various positive emotions, express desires and goals, and be done for its own sake (Anable \& Gatersleben, 2005; Diana, 2008; Handy, Weston, \& Mokhtarian, 2005; Loo, Corcoran, Mateo-Babiano, \& Zahnow, 2015; Milakis, Cervero, van Wee, \& Maat, 2015; Mokhtarian, in progress; Mokhtarian, Salomon, \& Singer, 2015; Ory \& Mokhtarian, 2005; Salomon \& Mokhtarian, 1998; Smith, 2017; Steg, 2005). For instance, traveling can make a person feel excited, relaxed, bold, comfortable, or happy. Alternatively, traveling can fulfill desires for adventure, control, or variety; help to express social status or self-identity; and improve self-confidence and mental health. Note that there may be considerable overlap between the affective and symbolic realms.

\subsubsection{Classification}

The three components of the PUT concept may not be completely independent and mutually exclusive, and the boundary between travel activities and travel experiences may be somewhat blurred. When instances of a PUT are difficult to classify, criteria offered in

${ }^{5}$ Certainly, most outdoor recreational activities are goal-directed (and thus, eudaimonic), whether for physical, mental, emotional (hedonic), and/or spiritual reasons. In reality, there may be only a few instances of personal travel as the activity (excluding travel as part of a job) with purely extrinsic or instrumental motivations: test-driving a car or bicycle with the intention to purchase it; or, operating a vehicle as part of a driver education course. 
Table 2.1 may help. In addition to understanding the spectrum of motivations for travel, one might consider language: Activities involve doing something (“_ing”), while experiences involve experiencing or having travel express something ("feeling _" or "being _"). Agency may also matter: Travelers tend to have more control over what activities they do than what they experience. A final way to distinguish between the travel activity and travel experience components is that the fundamental traits of the former can often be conceptually separated from travel (i.e., done in a nontravel setting), while the characteristics of the latter are fundamentally intertwined with travel.

Consider someone snoozing as a transitional buffer on the way home from work. A person can likely snooze almost as well while traveling as an office or at home, so this is more likely a travel activity (resting) than an emotional benefit of the travel experience (becoming less stressed). Now, consider a family traveling through a national park while on a cross-country trip. While activities are probably being conducted (window-gazing, taking pictures), most of the benefits likely derive from the thrill of seeing pleasant scenery or wild animals or feeling connected to nature. The curious case of the exercise and physical activity benefits of walking and bicycling poses another classification challenge. In many ways, it makes sense to classify these benefits as from travel-based multitasking: Many people walk and cycle for transportation specifically to make productive use of their travel time, potentially substituting an active commute for a gym membership. In other ways, these benefits seem more experiential: For some, traveling by foot or bike is fun, enjoyable, and a means to better mental and/or physical health.

One potential reconciliation for these difficult cases is to conceptually allow effects to occur across PUT domains, specifically, for travel activities to influence positive travel 
experiences. For the family in the national park, the act of window-gazing likely unlocks experiential benefits that would be greater than if these travelers were looking at the same scenes on a smartphone. In the case of walking and bicycling, while the act of exercising may be a travel activity with time use benefits, any enjoyment or positive affect from being physically active or fulfillment and eudaimonia from feeling healthier could qualify as aspects of the travel experience. While imperfect, the criteria of Table 2.1 offer a useful guide towards a stronger conceptualization and categorization of PUT factors.

\subsection{How can a positive utility of travel be measured?}

As this review aims to guide research on the PUT concept, it must offer not only conceptual but also methodological commentary. This section supplements a deeper theoretical understanding of the PUT concept by organizing and critiquing measurement methods and corresponding empirical evidence. In Table 2.2 and the sections below, subject areas are identified and categorized as to whether they are related to travel activities, travel experiences, or general PUT. Each topic is then summarized with respect to its evidence and methods, its relationship with the PUT notion, and alternative explanations. Below, Figure 2.1 condenses these conclusions about each approach's convincingness as an indicator of a PUT. This section builds upon other efforts connecting many topics to the PUT concept (e.g., Salomon \& Mokhtarian, 1998; Mokhtarian \& Salomon, 2001; Mokhtarian, 2014), but any errors or omissions are the fault of the author alone. 
Table 2.2 Types of measurement methods and evidence of a PUT

\begin{tabular}{|c|c|c|c|}
\hline Topic & Description & Relationship to a PUT & Alternative explanations \\
\hline \multicolumn{4}{|l|}{ General PUT } \\
\hline Travel time budgets & $\begin{array}{l}\text { Aggregate average daily travel } \\
\text { durations are constant across time } \\
\text { and space, roughly one hour/day. }\end{array}$ & $\begin{array}{l}\text { People may desire a certain amount of } \\
\text { daily travel, which could generate } \\
\text { stable average travel times. }\end{array}$ & $\begin{array}{l}\text { Tradeoffs between land/housing and transportation costs; } \\
\text { urban spatial structures; transportation technological } \\
\text { advances; other time constraints. }\end{array}$ \\
\hline $\begin{array}{l}\text { Desired travel } \\
\text { amounts }\end{array}$ & $\begin{array}{l}\text { People report an ideal commute time } \\
\text { greater than zero, or a desire to } \\
\text { travel more than they currently } \\
\text { do. }\end{array}$ & $\begin{array}{l}\text { People may positively value aspects } \\
\text { of their commutes, or like traveling } \\
\text { enough to want to travel more. }\end{array}$ & $\begin{array}{l}\text { Willingness to travel an acceptable amount; } \\
\text { rationalization of actual travel amount; situational } \\
\text { constraints; impossibility of zero travel time; } \\
\text { confounding of activities and travel. }\end{array}$ \\
\hline Excess commuting & $\begin{array}{l}\text { On aggregate, actual commute } \\
\text { lengths are longer than theoretical } \\
\text { minimum commute lengths. }\end{array}$ & $\begin{array}{l}\text { People may like aspects of their } \\
\text { commutes, e.g., as a buffer between } \\
\text { home and work. }\end{array}$ & $\begin{array}{l}\text { Multiple-worker households; job uncertainty; mismatch } \\
\text { of job requirements and employee skills; housing } \\
\text { transaction costs; mismatch of income and housing } \\
\text { costs; neighborhood amenities; transportation system } \\
\text { subsidies; increasing importance of nonwork travel. }\end{array}$ \\
\hline $\begin{array}{l}\text { Non-shortest path } \\
\text { route choice }\end{array}$ & $\begin{array}{l}\text { A subset of excess travel, where } \\
\text { chosen routes are not the shortest, } \\
\text { fastest, or cheapest. }\end{array}$ & $\begin{array}{l}\text { People may want to travel more than } \\
\text { necessary because they like it, or } \\
\text { they may choose longer but more } \\
\text { enjoyable and pleasant routes. }\end{array}$ & $\begin{array}{l}\text { Avoiding unpleasant or dangerous conditions; dealing } \\
\text { with unreliable transportation networks; habit; } \\
\text { unaware of superior alternatives. }\end{array}$ \\
\hline $\begin{array}{l}\text { Excess travel } \\
\text { indicators }\end{array}$ & $\begin{array}{l}\text { People sometimes travel longer or } \\
\text { farther than necessary. }\end{array}$ & $\begin{array}{l}\text { People like to travel or get enjoyment } \\
\text { from traveling longer or farther. }\end{array}$ & $\begin{array}{l}\text { Destinations motivate behavior; habits; poor trip } \\
\text { planning; lack of information; survey response bias. }\end{array}$ \\
\hline $\begin{array}{l}\text { Coping with } \\
\text { congestion }\end{array}$ & $\begin{array}{l}\text { Travel behavior responses to } \\
\text { congestion-reducing policies are } \\
\text { less-sensitive than expected. }\end{array}$ & $\begin{array}{l}\text { People may enjoy parts of their } \\
\text { commutes and do not mind a little } \\
\text { congestion. }\end{array}$ & $\begin{array}{l}\text { Making other less-constrained changes: accepting costs; } \\
\text { changing travel time, mode, or route; buying time or } \\
\text { productivity at home; changing work schedules or } \\
\text { locations; changing employment status; or moving. }\end{array}$ \\
\hline Telecommuting & $\begin{array}{l}\text { Telecommuting levels are lower } \\
\text { than expected. }\end{array}$ & $\begin{array}{l}\text { People may like their commute, at } \\
\text { least a little, or they may value } \\
\text { travel as transition between work } \\
\text { and home. }\end{array}$ & $\begin{array}{l}\text { Lack awareness; misunderstanding; lack employer or } \\
\text { manager support; unsuitable job; high cost of } \\
\text { technology; need for personal interaction; lower } \\
\text { productivity at home; lack discipline; risk aversion. }\end{array}$ \\
\hline $\begin{array}{l}\text { Information and } \\
\text { communications } \\
\text { technologies }\end{array}$ & $\begin{array}{l}\text { ICTs and travel may be more } \\
\text { complementary than } \\
\text { supplementary. }\end{array}$ & $\begin{array}{l}\text { People may have a desired travel time } \\
\text { budget, or may like to travel. }\end{array}$ & $\begin{array}{l}\text { No ICT substitute; ICT not feasible, desirable, or a } \\
\text { replacement; saves time/money for other activities; } \\
\text { makes travel cheaper or more efficient. }\end{array}$ \\
\hline
\end{tabular}




\begin{tabular}{|c|c|c|c|}
\hline Topic & Description & Relationship to a PUT & Alternative explanations \\
\hline Teleportation test & $\begin{array}{l}\text { Some people choose commuting } \\
\text { over teleporting if given the } \\
\text { chance. }\end{array}$ & $\begin{array}{l}\text { People like to travel or benefit from } \\
\text { the time or experience of traveling. }\end{array}$ & $\begin{array}{l}\text { Teleportation as unknown technology; zero travel time } \\
\text { considered unrealistic or impossible. }\end{array}$ \\
\hline $\begin{array}{l}\text { Value of travel time } \\
\text { savings }\end{array}$ & $\begin{array}{l}\text { Negative values imply people are } \\
\text { willing to pay for longer trips. }\end{array}$ & $\begin{array}{l}\text { People like travel so much they will } \\
\text { pay for more; travel is valued for its } \\
\text { own sake or ultraproductive. }\end{array}$ & $\begin{array}{l}\text { Distributional assumptions; nonlinear responses to travel } \\
\text { time; stated preference survey design issues. }\end{array}$ \\
\hline \multicolumn{4}{|l|}{ Travel activities } \\
\hline $\begin{array}{l}\text { Travel-based } \\
\text { multitasking }\end{array}$ & $\begin{array}{l}\text { Many people conduct activities } \\
\text { while traveling. }\end{array}$ & $\begin{array}{l}\text { People must benefit from } \\
\text { multitasking; otherwise, why do it? }\end{array}$ & $\begin{array}{l}\text { Coping mechanism to reduce disutility; activities } \\
\text { required by others (coupling or authority constraints). }\end{array}$ \\
\hline Usefulness of travel & $\begin{array}{l}\text { Some people report travel time as } \\
\text { mostly useful or very worthwhile. }\end{array}$ & $\begin{array}{l}\text { People are making use of their travel } \\
\text { time by multitasking. }\end{array}$ & $\begin{array}{l}\text { Answers partially reflecting enjoyment or satisfaction } \\
\text { from the travel experience. }\end{array}$ \\
\hline \multicolumn{4}{|l|}{ Travel experiences } \\
\hline $\begin{array}{l}\text { Subjective well- } \\
\text { being (in the } \\
\text { travel domain) }\end{array}$ & $\begin{array}{l}\text { People have positive responses to } \\
\text { scales measuring subjective well- } \\
\text { being during/after travel. }\end{array}$ & $\begin{array}{l}\text { The travel experience encourages } \\
\text { positive feelings and provides a } \\
\text { means to achieve broader goals. }\end{array}$ & Self-selection; positive overall subjective well-being. \\
\hline $\begin{array}{l}\text { Affective responses } \\
\text { to travel }\end{array}$ & $\begin{array}{l}\text { People have positive emotions or at } \\
\text { least lack negative emotions as a } \\
\text { result of traveling. }\end{array}$ & $\begin{array}{l}\text { Travel evokes positive emotions, so } \\
\text { something about the experience is } \\
\text { valuable. }\end{array}$ & $\begin{array}{l}\text { Positive emotions in general; other activities even more } \\
\text { positive than travel. }\end{array}$ \\
\hline Travel liking & $\begin{array}{l}\text { Some people report that they like to } \\
\text { travel using certain modes or in } \\
\text { certain situations. }\end{array}$ & $\begin{array}{l}\text { Aspects of the travel experience make } \\
\text { traveling enjoyable or fulfilling. }\end{array}$ & $\begin{array}{l}\text { Confounding travel liking with liking of the destination } \\
\text { activity. }\end{array}$ \\
\hline $\begin{array}{l}\text { Satisfaction with } \\
\text { travel }\end{array}$ & $\begin{array}{l}\text { People report being satisfied or at } \\
\text { least not unsatisfied with travel. }\end{array}$ & $\begin{array}{l}\text { People are content with their amount } \\
\text { and/or quality of travel. }\end{array}$ & $\begin{array}{l}\text { Satisfaction reference levels more realistic than ideal; } \\
\text { adaptation to routine; rationalization. }\end{array}$ \\
\hline $\begin{array}{l}\text { Noninstrumental } \\
\text { reasons for } \\
\text { traveling }\end{array}$ & $\begin{array}{l}\text { Some people report traveling for } \\
\text { reasons not related to getting } \\
\text { somewhere. }\end{array}$ & $\begin{array}{l}\text { These noninstrumental motivations } \\
\text { are due to the experience of } \\
\text { traveling. }\end{array}$ & $\begin{array}{l}\text { Justification or rationalization of past travel; response } \\
\text { inconsistencies; blurred lines between affective and } \\
\text { instrumental motives. }\end{array}$ \\
\hline
\end{tabular}




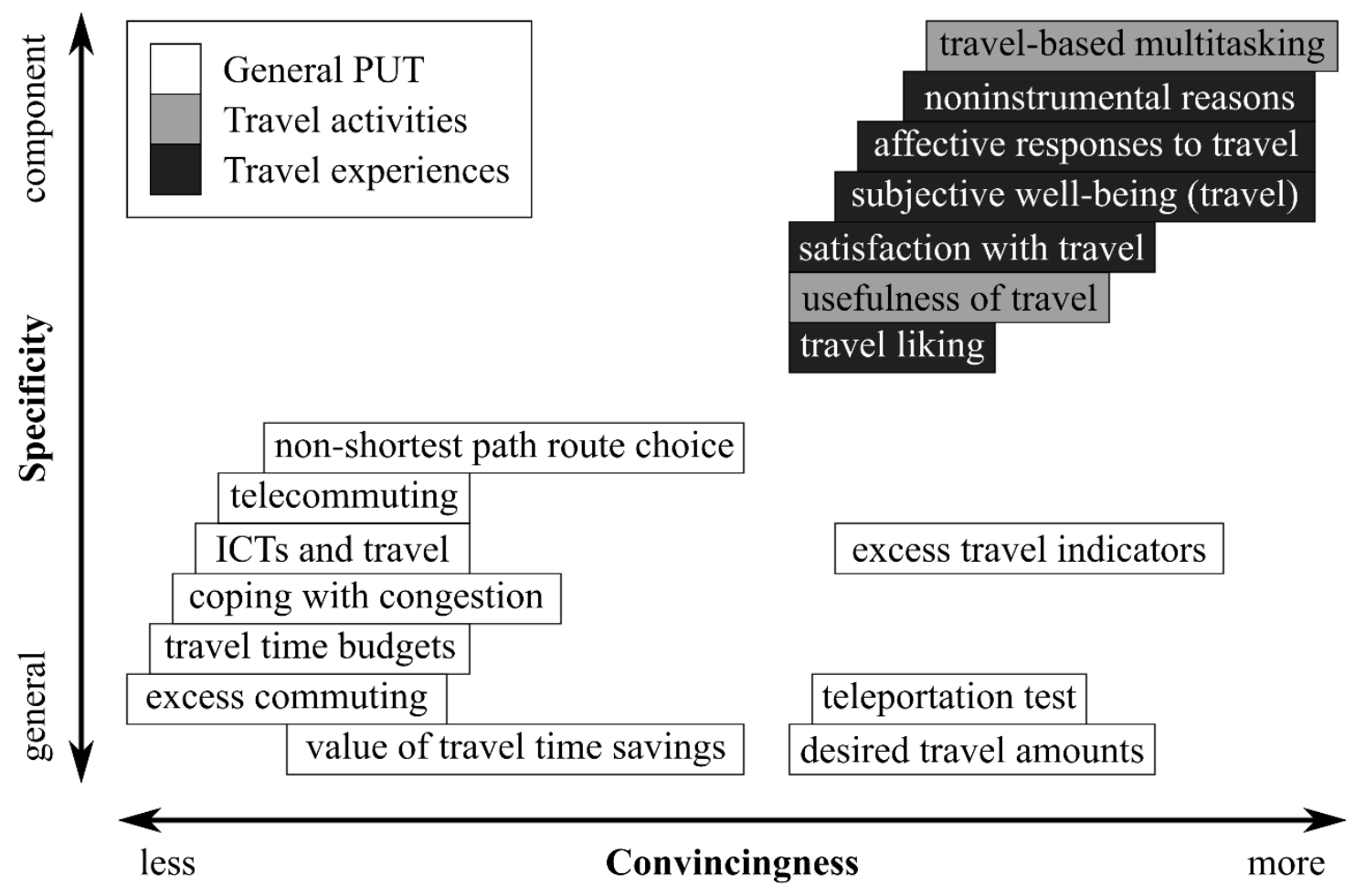

Figure 2.1 Convincingness and specificity of types of PUT methods and evidence

\subsubsection{General PUT}

Many approaches may point towards evidence of a PUT in general, not specific to any component. Usually, these are findings could be consistent with but are not conclusive of PUT, because the underlying mechanism linking the PUT concept to the evidence may be unclear or unobserved. As a result, many of these methods - especially those that rely on aggregate observations - are considered to provide less convincing evidence of a PUT, since there may be stronger alternative explanations. 


\subsubsection{Travel time budgets}

The travel time budget concept suggests that the time individuals spend traveling or commuting is relatively constant, roughly just over one hour per day (Mokhtarian \& Chen, 2004). This number is popularly called Marchetti's constant or wall (Newman \& Jennings, 2008). Supporters suggest aggregate travel time expenditures are relatively stable across both space (in different countries or cultures) and time (throughout history), and discuss how transportation technologies have enabled increases in speeds and distances traveled while holding travel times constant (Hupkes, 1982; Marchetti, 1994; Mumford, 1961). Explanations often involve PUT-related ideas, ranging from liking or intrinsically valuing travel to a fundamental human instinct for territory expansion. However, there is little evidence for constant travel time and money budgets at an individual level (Mokhtarian \& Chen, 2004). Besides the issue of confusing an aggregate trend for an individual behavior, there are other explanations for travel time budgets. They may be the natural result of tradeoffs between generalized commuting costs and housing costs; alternatively, constraints on time spent in other daily activities may naturally limit the time people can spend commuting. Evidence of travel time budgets may not say much about the existence of a PUT.

\subsubsection{Desired travel amounts}

A more convincing approach to the individual travel time budget concept is to ask questions about desired travel amounts, either absolute or relative. Several studies have asked travelers to report their ideal travel times to work or school and/or their desires for more or less of different types of travel (Choo, Collantes, \& Mokhtarian, 2005; Handy et 
al., 2005; He, Zhao, \& He, 2016; Milakis et al., 2015; Mokhtarian \& Salomon, 1999; Mokhtarian \& Salomon, 2001; O’Fallon \& Wallis, 2012; Páez \& Whalen, 2010; Redmond \& Mokhtarian, 2001; Russell, 2012; Russell \& Mokhtarian, 2015; Watts \& Urry, 2008). Average ideal commute times range from 10 to 30 minutes, often near 15 minutes; only roughly $1-3 \%$ of commuters desire zero minutes. A small but not insignificant proportion of people $(<10 \%)$ actually want to do more short-distance travel.

If travel is completely a disutility, absolute desired travel should be zero. Presumably, people with nonzero ideal travel times positively value travel for some reason. Similarly, people with positive relative desired mobility should have desired travel amounts in excess of current amounts. However, it is possible that respondents instead gave acceptable or realistic travel times (given situational constraints), rationalized actual travel times, or answered with desires for more activity participation (Choo et al., 2005; Redmond \& Mokhtarian, 2001). Although promising indicators of general PUT, questions about desired travel amounts should be worded to avoid these potential confounders and response biases.

\subsubsection{Excess commuting}

The concept of excess travel suggests that people travel more than necessary to conduct activities at destinations. Excess or wasteful commuting (Hamilton, 1989; Hamilton \& Röell, 1982; Kanaroglou, Higgins, \& Chowdhury, 2015; Small \& Song, 1992; White, 1988) is the difference between an aggregate amount of commuting (distance or time) observed in a region and a theoretical minimum commute amount, usually obtained from an optimization model of urban location choices. Certainly, commuting amounts 
should be greater than optimal if commuters positively value some aspects of their journeys to work, be it as transition time, time alone, or time to be productive. However, there are more compelling explanations for excess commuting: competing demands of multipleworker households; transaction costs of moving or changing jobs; job instability; transportation subsidies; neighborhood amenities; and the increasing share and importance of nonwork travel (Ma \& Banister, 2006). Given this, the contribution of a PUT to this type of excess commuting is likely small.

\subsubsection{Non-shortest-path route choice}

Another example of excess travel is non-shortest-path route choice. Indeed, there is evidence that, when driving (Bovy \& Stern, 1990), walking (Agrawal, Schlossberg, \& Irvin, 2008), or bicycling (Broach, Dill, \& Gliebe, 2012), people may not always take the shortest, fastest, or cheapest route to their destination. PUT may be at play if travelers want to travel farther than necessary or if they choose more scenic or enjoyable but out-of-theway paths. However, excess travelers may instead be motivated by minimizing more general measures of travel disutility, including a lack of reliability, safety, or security, especially for nonmotorized travel (Singleton \& Wang, 2014). Without traveler perceptions of the negative and positive aspects of route choice, it may be unreasonable to conclude that non-shortest-path route choice constitutes direct evidence of PUT.

\subsubsection{Excess travel indicators}

Stronger evidence for PUT-related motivations behind excess travel comes from direct questioning of people's reasons for excess travel. These excess travel indicators 
include questions about traveling: to explore new places, by a new route to a familiar destination, to a more distant destination than necessary, etc. (Cao, Mokhtarian, \& Handy, 2009; Diana, 2008; Handy et al., 2005; Mokhtarian \& Salomon, 2001). Results indicate $30-60 \%$ of people occasionally engage in some form of excess travel. Nevertheless, reported excess travel could instead be the result of habits, poor trip planning, misperceptions, or a lack of information (Handy et al., 2005). Future studies on excess travel should carefully choose indicators that have a closer conceptual link to PUT-related motivations: e.g., out of the way for pleasant scenery, with no particular destination in mind, to clear your head, just for the fun of it, to show off a means of transportation.

\subsubsection{Coping with congestion}

Automobile congestion reduction policies, particularly travel demand management strategies, have had lower than expected effects for a variety of reasons (Giuliano \& Small, 1995). The PUT concept could be one explanation: If people positively value travel, they may be less sensitive to behavior change strategies to reduce travel demand. In fact, some research suggests people who like to travel are less likely to adopt travel-reducing or more likely to adopt travel maintaining/increasing strategies to deal with congestion (Cao \& Mokhtarian, 2005a; Choo \& Mokhtarian, 2008; Clay \& Mokhtarian, 2004). On the other hand, other non-PUT-related congestion coping mechanisms may also act: accepting the

costs, changing travel patterns, changing work schedules or locations, changing employment status, buying time or productivity at home, and moving (Cao \& Mokhtarian, 2005b; Salomon \& Mokhtarian, 1997). 


\subsubsection{Telecommuting}

One congestion coping strategy is telecommuting: working from home. While telecommuting continues to increase (US Census Bureau, 2014), adoption rates are still far below (perhaps overly) optimistic forecasted levels (Nilles, 1988). The PUT idea could explain these low levels: People who find benefits to commuting may be less motivated to telecommute. However, other stronger factors likely constrain telecommuting: a lack of awareness, employer or manager discouragement, unsuitable jobs, technological costs, lower productivity, etc. (Mokhtarian \& Salomon, 1994). Unfortunately, the magnitude of PUT's role on telecommuting adoption is unknown because most telecommuting studies (with exceptions: e.g., Mokhtarian \& Salomon, 1997) do not investigate PUT-related aspects.

\subsubsection{Information and communications technologies}

The PUT concept could also play a role in defining the interactions between travel and the teleactivities (like telecommuting) made possible by information and communications technologies (ICTs). There are four fundamental relationships between ICT and travel: substitution (ICT use replaces travel), generation/complementarity (ICT use increases travel), modification (ICT use changes travel characteristics), and neutrality (no effect) (Mokhtarian, 1990; Mokhtarian, 2002; Niles, 1994; Salomon, 1985; Salomon, 1986). Reviews find evidence of short-term substitution for telecommuting but neutrality or complementarity for teleshopping and teleleisure (Andreev, Salomon, \& Pliskin, 2010; Mokhtarian \& Salomon, 2002); there is recent evidence that telecommuting may even increase nonwork travel (He \& Hu, 2015; Kim, Choo, \& Mokhtarian, 2015; Zhu, 2012). 
Low substitution or complementarity between travel, telecommuting, and other ICT-enabled teleactivities could be consistent with a PUT: "To the extent the autotelic role is in play, individuals will be much less inclined to adopt ICT substitutes for travel" (Mokhtarian, 2009, p. 3). Yet again, other explanations may be stronger and more convincing: Many activities have an inferior or no ICT substitute; and ICTs can make travel cheaper or save time for other activities, thus stimulating more travel (Mokhtarian, 2009). ICTs do enable travel-based multitasking (see the section on Travel activities).

\subsubsection{Teleportation test}

One way to examine if a PUT influences telecommuting choices or ICT/travel substitution is to conduct the teleportation test (Mokhtarian \& Salomon, 2001; Russell \& Mokhtarian, 2015). This simple stated choice experiment asks whether people would be willing to instantaneously teleport to their desired destination (usually work) if the technology existed. It has been used in a handful of questionnaire and focus group studies (Diana, 2008; Handy et al., 2005; Jain \& Lyons, 2008; O’Fallon \& Wallis, 2012; Russell, 2012; Russell \& Mokhtarian, 2015; Watts \& Urry, 2008) with similar results: Roughly $75 \%$ of people report wanting to teleport. Since it eliminates many constraints of teleactivities (see previous sections), the teleportation test theoretically isolates positive travelrelated aspects. However, some of the $25 \%$ of people who would rather travel than teleport might be apprehensive of an unknown technology or (as with ideal travel time) reluctant to choose an impossible alternative. Russell and Mokhtarian (2015) discuss options for including the teleportation test in a questionnaire survey to more deeply probe motivations — including those related to the PUT concept—-for or against teleportation. 


\subsubsection{Value of travel time savings}

Conceptually, the value of travel time savings (VTTS) — the willingness to pay for a marginal decrease in travel time — should be a strong indicator of a PUT. Derived from individual-level revealed or stated preference data, VTTS has a useful economic interpretation. Positive VTTS means travel time is a disutility. Negative VTTS implies that people would be willing to pay money to increase their travel time; they must enjoy traveling at the margin. Zero VTTS suggests the loss in time is exactly offset by positive aspects of traveling. Intuitively, findings of VTTS $\leq 0$ should be direct evidence of a PUT. Indeed, studies using mixed logit mode choice models have found a not insignificant group of people (10-40\%) with zero (Cirillo \& Axhausen, 2006; Fosgerau, 2006; Richardson, 2003) or negative (Algers, Bergström, Dahlberg, \& Lindqvist Dillén, 1998; Cirillo \& Axhausen, 2006; Ettema \& Verschuren, 2007) VTTS. Yet, according to time allocation and valuation theory, negative VTTS is impossible; VTTS can be zero at best (Bates, 1987; DeSerpa, 1971; Evans, 1972; González, 1997; Hess et al., 2005; Jara-Díaz, 2000; Jara-Díaz \& Guevara, 2003). As Evans (1972) explains (reiterated by González, 1997): Even if “the time the consumer wishes to spend travelling is greater than the time he must spend traveling...Any small increase or decrease in the time he must spend traveling will not alter his allocation of time" (p. 11).

Some scholars try to explain these theoretically impossible results, suggesting "findings of negative VTTS in the literature are [likely] economic artifacts" (Hess et al., 2005, p. 229), due to modeling assumptions like the choice of random coefficient distributions in mixed logit models, misspecified utility equations that do not consider nonlinear responses to level-of-service variables, or poor quality data due to stated 
preference survey response problems (Cirillo \& Axhausen, 2006; Fosgerau, 2006; Hess et al., 2005; Pinjari \& Bhat, 2006; Richardson, 2003; Sillano \& Ortúzar, 2005). Another explanation, more positive for a PUT, is that findings of negative VTTS result from omitting relevant variables (like unobserved travel activities and positive experiences) that are correlated with travel time (Hess et al., 2005; Salomon \& Mokhtarian, 1998; Singleton \& Clifton, 2015). Regardless, negative VTTS is not required to document a PUT; changes in VTTS due to PUT-related attitudes (Abou-Zeid, Ben-Akiva, Bierlaire, Choudhury, \& Hess, 2010) can indicate its presence. Unfortunately, it is extremely challenging to empirically extract the desired marginal utility of travel time from within an estimate of VTTS (Jara-Díaz \& Guevara, 2003). More theoretical and empirical research is needed before a PUT can be consistently and directly measured using the value of travel time savings, although it could be a useful indicator of a PUT in some situations.

\subsubsection{Travel activities}

Additional sources of empirical evidence related to the travel activity aspect of the PUT concept. Intuitively, that these findings are strong evidence of a (perhaps weaker) form of a PUT. Most of these studies fall within travel-based multitasking.

\subsubsection{Travel-based multitasking}

Conceptually, any sort of activity engagement during travel should be positively valued; otherwise, why do it? Although activities conducted while simultaneously traveling are convincing examples of a PUT, this form of the PUT concept may be weaker than that arising from an enjoyment of the travel experience. Travel activity engagement 
may simply reduce the disutility of what would otherwise be a burdensome trip (Mokhtarian, 2014), although this is still a more minor expression of the PUT concept. One exception to the axiom of positively valued travel activities is an obligatory activity due to coupling or authority constraints (e.g., employer-mandated work on a business trip; taking a phone call about a problem at home), which can be less desirable and may even generate stress (Kenyon \& Lyons, 2007). Nevertheless, it seem safe to suggest that most instances of travel-based multitasking are indeed expressions of a PUT.

Travel-based multitasking is a subset of multitasking and belongs to a broader literature on time use and human performance. Historically, multitasking studies have suffered from limitations: inconsistent definitions of multitasking; confounding multitasking with polychronicity (the preference for multitasking); confusions between the shares of resources and shares of time dedicated to different activities; measuring only a few activities; differences distinguishing primary and secondary activities; and issues with classifying distinct activities (Circella et al., 2012; Kenyon, 2010; König \& Waller, 2010). For the purposes of this study, traveling is considered to be the primary activity ${ }^{6}$ and all instances of activity participation during travel to be multitasked secondary activities.

There were few studies of travel-based multitasking until recently (Kenyon \& Lyons, 2007). Studies use different data collection methods, including field observations (Guo, Derian, \& Zhao, 2015; Ohmori \& Harata, 2008; Russell et al., 2011; Timmermans

\footnotetext{
${ }^{6}$ A few scholars have wondered whether some forms of passive travel are even activities (Kenyon, 2010, p. 54; Circella et al., 2012, p. 84). For instance: The major conceptual difference between riding on/in a train/bus/taxi and cleaning clothes using a home washing machine is that passenger travel requires copresence of the traveler and the means of transportation at all times. Otherwise, both activities involve the use of technology toward some end goal (going somewhere vs. cleaning clothes) and require an initial action (boarding the vehicle vs. loading and starting the machine) but little-to-no input throughout the duration. Most people would be more likely to consider the first to be an activity than the second.
} 
\& van der Waerden, 2008; van der Waerden, Timmermans, \& van Neerven, 2009), questionnaire surveys and activity diaries (Berliner, Malokin, Circella, \& Mokhtarian, 2015; Diana, 2008; Ettema, Friman, Gärling, Olsson, \& Fujii, 2012; Ettema \& Verschuren 2007; Frei, Mahmassani, \& Frei, 2015; Guo et al., 2015; Kenyon, 2008; Kenyon \& Lyons, 2007; Keseru et al., 2015; Lyons, Jain, \& Holley, 2007; Lyons et al., 2013; Lyons, Jain, \& Weir, 2016; Malokin et al., 2015; Mokhtarian, Papon, Goulard, \& Diana, 2015; Ohmori \& Harata, 2008; Yosritzal, 2014; Zhang \& Timmermans, 2010), and interviews or focus groups (Handy et al., 2005; Jain \& Lyons, 2008). While observations can reduce response bias and more accurately capture activity durations, self-reported survey data may be more useful for studying PUT because activities may be more closely related to underlying motivations (recall the activities vs. items discussion). Most analyses focus on public transit passengers; few investigate multitasking while walking and bicycling (Circella et al., 2015). Across all modes, the majority of people appear to multitask in some way while traveling.

\subsubsection{Usefulness of travel}

A few multitasking studies also ask travelers to assess the value, worth, or usefulness of a trip (Circella et al., 2015; Frei et al., 2015; Lyons et al., 2007; Lyons et al., 2013; Susilo, Lyons, Jain, \& Atkins, 2012). Approximately 20-25\% of travelers report travel time to be very worthwhile or mostly useful; only $15-20 \%$ say travel time is mostly wasted time. While responses to these questions could be partially due to enjoyment or satisfaction from the travel experience, their wording (on a scale from useful to wasted) 
more likely measures a general assessment of travel time productivity due to activity engagement.

\subsubsection{Travel experiences}

Another area of research examines the experience of traveling itself, including instances where travel evokes or is intrinsically motivated by positive sensations, emotions, and purposes. While these methods may not be able to fully conclude the presence of a PUT or that the findings are caused by positive travel-experience-related mechanisms, overall, the evidence points more strongly towards their involvement. Many of these concepts are connected to subjective well-being.

\subsubsection{Subjective well-being (in the travel domain)}

Well-being is a broad concept with multiple dimensions (Nordbakke \& Schwanen, 2014), encompassing health, satisfaction, happiness, and quality of life. Subjective wellbeing (SWB) - a common psychological conceptualization of well-being - is typically decomposed into hedonic and eudaimonic aspects. As discussed earlier, hedonic SWB is related to preference satisfaction, utility, and feelings of happiness and pleasure; eudaimonic SWB is about finding purpose or meaning in life, personal growth, attaining goals, and achieving self-realization (De Vos et al., 2013). Hedonic SWB can be subdivided into three parts (Diener, 1984): positive and negative affect (short-term presence or absence of positive emotions or mood) and cognitive evaluation (long-term life satisfaction). A number of well-established scales exist for measuring SWB (De Vos et al., 2013; Ettema, Gärling, Olsson, \& Friman, 2010; Mokhtarian, in progress). 
Transportation and SWB are closely linked. Several reviews have summarized the evidence for and pathways of transportation's effects on well-being (Delbosc, 2012; Reardon \& Abdallah, 2013). Fewer researchers have emphasized the bidirectional relationship between SWB and travel behavior (De Vos et al., 2013), with directions of causality that can be difficult to disentangle (Mokhtarian, in progress). The PUT thesis presumes that travel experiences can positively affect short-term SWB, and that people may modify their travel behavior based on expectations of improved well-being (AbouZeid \& Ben-Akiva, 2014).

Ettema and colleagues have developed the Satisfaction with Travel Scale (STS) to measure hedonic SWB in the travel domain (Ettema et al., 2011). Based on earlier work and the Swedish Core Affect Scale (Västfjäll, Friman, Gärling, \& Kleiner, 2002), the STS includes nine items assessed on a seven-point semantic differential scale and has been shown to be a satisfactory measure of travel-based SWB (De Vos, Schwanen, Van Acker, \& Witlox, 2015; Friman, Fujii, Ettema, Gärling, \& Olsson, 2013). The STS and its variants have been applied to a number of different situations: travel plans, commute trips, leisure trips, and daily travel in general (De Vos, Mokhtarian, Schwanen, Van Acker, \& Witlox, 2016; De Vos et al., 2015; Ettema et al., 2012; Ettema et al., 2011; Olsson, Gärling, Ettema, Friman, \& Fujii, 2013; Smith, 2017; Ye \& Titheridge, 2017; Zhao \& Lee, 2013). While some findings of positive SWB in the travel domain could be due to self-selection or positive overall SWB, it seems more likely that they result from PUT-related aspects of the travel experience. 


\subsubsection{Affective responses to travel}

A number of studies have elicited responses about travelers' feelings, emotions, and moods as a result of a travel experience (Anable \& Gatersleben, 2005; Archer, Paleti, Konduri, Pendyala, \& Bhat, 2013; Duarte et al., 2010; Gatersleben \& Uzzell, 2007; Mokhtarian, Papon et al., 2015; Morris \& Guerra, 2015a, 2015b; Rhee, Kim, Lee, Kim, \& Lee, 2013; Thomas \& Walker, 2015). Questions about purely affective responses to travel have even been included in national representative samples like the French National Travel Survey and the American Time Use Survey. Despite conventional wisdom about commuting as a negative experience, over or nearly half of people are somewhat happy or find their commutes or a recent trip to be pleasant. Although affect during travel is less positive than during leisure, recreation, and volunteering, it tends to be more positive than during work and household maintenance activities. Certainly, a positive travel affect could be influenced by a positive general affect; however, these results at least suggest that travel is not completely a disutility.

\subsubsection{Travel liking}

One of the first measures of the PUT concept asked about people's general affinity for travel, or travel liking (Mokhtarian \& Salomon, 2001). Travel liking questions presumably address an affective response to travel (Ory \& Mokhtarian, 2009), yet they are prevalent enough (Curry, 2000; Gatersleben \& Uzzell, 2007; Mokhtarian, Papon et al., 2015; Mokhtarian \& Salomon, 2001; Ory \& Mokhtarian, 2005; Ory et al., 2004; Turcotte, 2006) to warrant their own discussion. Depending on the mode or trip purpose, a substantial portion of people like to travel, and most people do not dislike traveling. In fact, only 30- 
$40 \%$ of people dislike commuting, the least enjoyed type of short-distance travel. One issue with travel liking is that people may confound it with liking the destination activity; also, the question offers little insight into why people like to travel. Nevertheless, if shown to be related to other measures of travel affect, travel liking could be a useful single question for future research on the PUT concept.

\subsubsection{Satisfaction with travel}

Instead of using the STS, some studies simply ask one question about overall satisfaction with travel. These direct questions are more likely to evoke a cognitive evaluation than assess positive/negative affect (De Vos et al., 2013), although they may also unintentionally capture satisfaction with travel-based multitasking. While most studies are retrospective (Abou-Zeid \& Ben-Akiva, 2011; Archer et al., 2013; Mao, Ettema, \& Dijst, 2015; St-Louis, Manaugh, van Lierop, \& El-Geneidy, 2014; Susilo et al., 2017), some are also prospective (Milakis et al., 2015; Wachs, Taylor, Levine, \& Ong, 1993; Young \& Morris, 1981), asking about satisfaction with hypothetical commute times (typically peaking around 15 minutes). As with travel affect, roughly half of people are satisfied with their commutes or a recent trip, and travel satisfaction is higher than work satisfaction (but lower than for most other out-of-home activities). Travel satisfaction questions - like those about desired travel amounts—are weaker indicators of a PUT because people may rationalize unsatisfactory choices, adapt to a routine, or answer bounded by what is realistic. 


\subsubsection{Noninstrumental reasons for traveling}

Another way to examine travel experience factors is to directly ask people why they travel, eliciting affective/hedonic and (especially) symbolic/eudaimonic motivations that go beyond instrumental reasons (e.g., travel time, cost, convenience, reliability, effort). Methods include qualitative interviews or focus groups (Gardner \& Abraham, 2007; Handy et al., 2005; Hiscock, Macintyre, Kearns, \& Ellaway, 2002; Jain \& Lyons, 2008; Mann \& Abraham, 2006) as well as questionnaire surveys (Ellaway, Macintyre, Hiscock, \& Kearns, 2003; Loo et al., 2015; Steg, 2005). Many studies focus on motivations for driving and car use (Gatersleben, 2014; Steg, 2005; Zhao \& Zhao, 2015), while others are founded in a sociological perspective of studying mobilities (Watts \& Urry, 2008) and the individual, localized, social, and cultural environments in which travel takes place. Although uncovering true travel motivations can be challenging due to post-hoc justifications, response inconsistencies, and blurred lines (Mann \& Abraham, 2006), these kinds of questions appear to be a useful tool for examining hedonic and eudaimonic motivations related to the PUT concept.

\subsection{What are determinants of a positive utility of travel?}

Given evidence for the existence of a PUT, it then becomes relevant to investigate what factors affect or are associated with the PUT concept and its components. A statistical analysis of these PUT determinants is important - to isolate PUT's unique effects on travel behavior from the confounding effects of its correlates - and has practical implications: If more easily measured variables strongly predict PUT, they can be used in place of the intensive PUT data collection methods described in the previous section. This subject could 
demand its own full review, so only a brief summary is presented here; additional information is contained in Chapter 4 and Chapter 5. The determinants of PUT fall into two broad categories: travel characteristics and personal characteristics.

Unsurprisingly, trip and travel characteristics influence PUT. Foremost are modal effects, as expected: There are inherent differences in how each mode facilitates multitasking, influences travel experiences, and acts as a symbol. Activities like reading or sleeping are done more often by (train, bus, or car) passengers; listening, talking, and looking at scenery are common among (car or bicycle) operators (Berliner et al., 2015; Circella et al., 2015; Ettema \& Verschuren, 2007; Keseru et al., 2015; Russell et al., 2011). Experiences walking and bicycling tend to be more positive than for automobile travel, which is more positive than riding transit (Anable \& Gatersleben, 2005; De Vos et al., 2016; De Vos et al., 2013; De Vos et al., 2015; Duarte et al., 2010; Ettema et al., 2011; Friman et al., 2013; Gatersleben \& Uzzell, 2007; Mao et al., 2015; Morris \& Guerra, 2015a; Olsson et al., 2013; Rhee et al., 2013; Smith, 2017; St-Louis et al., 2014; Susilo et al., 2017; Thomas \& Walker, 2015; Ye \& Titheridge, 2017; Zhao \& lee, 2013). Travel time also influences PUT in complicated directions. Longer trips often have higher or more active activity participation (Frei et al., 2015; Lyons et al., 2007; Lyons et al., 2013; Ohmori \& Harata, 2008; Timmermans \& van der Waerden, 2008; Zhang \& Timmermans, 2010), due to the higher quality of longer activity durations or people with longer trips being more inclined to make use of that time. Yet, satisfaction with the travel experience tends to decrease with longer trip lengths (Milakis et al., 2015; Morris \& Guerra, 2015b; Olsson et al., 2013; Ory \& Mokhtarian, 2005, 2009; Rasouli \& Timmermans, 2014a; Smith, 2017; Susilo et al., 2017; Turcotte, 2006; Wachs et al., 1993). Alternatively, satisfaction may be 
a nonlinear function of travel time, because several studies find a peak at around 15 minutes (Milakis et al., 2015; Wachs et al., 1993; Young \& Morris, 1981). Trip purpose also seems to affect PUT. Commutes tend to involve more "productive" uses of time (i.e., reading or writing) (Keseru et al., 2015; Lyons et al., 2007; Lyons et al., 2013); however, the commuting experience seems to be less-positively valued than other trip purposes (Mokhtarian, Papon et al., 2015; Mokhtarian \& Salomon, 2001; Morris \& Guerra, 2015a; Ory \& Mokhtarian, 2005). Although a purpose-specific PUT might result from a respondent confounding multitasking benefits or destination enjoyment with travel enjoyment, valid reasons—such as preparation for or anticipation of the destination activity—may also be involved.

Interestingly, few demographic or socioeconomic personal characteristics are consistently associated with PUT measures. Most studies find only small differences in travel-based multitasking by gender and age. Women may be slightly more likely to spend some time talking with others (Keseru et al., 2015; Russell et al., 2011; Timmermans \& van der Waerden, 2008), and younger people may do more activities using higher-tech ICT devices like smartphones (Berliner et al., 2015; Guo et al., 2015; Russell et al., 2011). For travel experience factors, age seems significant. Older travelers tend to have a more positive affect and higher satisfaction or SWB for a particular trip (Archer et al., 2013; Jakobsson Bergstad et al., 2011; Mokhtarian, Papon et al., 2015; Olsson et al., 2013; Ory \& Mokhtarian, 2005; Rasouli \& Timmermans, 2014a, 2014b; St-Louis et al., 2014; Ye \& Titheridge, 2017). The importance of age may point to broader life stage, lifestyle, or cultural influences. Some traveler attitudes and personalities appear to be more closely linked to measures of PUT than typical sociodemographic measures. Polychronic people 
may conduct activities that are more active and consider travel to be more useful (Berliner et al., 2015). Proenvironmental attitudes have been related to nonautomobile travel liking (Ory \& Mokhtarian, 2005). These results suggest the importance of controlling for attitudes when studying the PUT concept, and the need for additional research into personal characteristics that might explain variations in PUT measures.

\subsection{How do positive utilities of travel affect travel behavior?}

An ultimate objective of research on the PUT concept is to examine its potential influences on travel behavior. Unfortunately, there is relatively little work on this to date. Few studies described above related PUT measures to travel behaviors, and most of those analyzed either travel activity factors or travel experience factors of PUT, not both.

A handful of studies have investigated travel behavior impacts of travel-based multitasking. Several have focused on train travel. In a revealed preference study in California, the installation of free WiFi was associated with a modest increase in train passengers' frequency of use (Dong, Mokhtarian, Circella, \& Allison, 2015). Two Netherlands-based stated preference studies found that advantages of train over car travel (sitting down, table space, internet access, and quiet compartments) had no impact on stated mode choice (van der Waerden, Kemperman, Timmermans, \& van Hulle, 2010), but that polychronic commuters who listened to music were less sensitive to travel time (Ettema \& Verschuren, 2007). Looking across multiple modes, a newer revealed preference study in the same region of California (Neufeld \& Mokhtarian, 2012) identified impacts of travelbased multitasking on commute mode choice. Malokin et al. (2015) found that perceptions of each mode's multitaskability were positively associated with choosing that mode, and 
that a propensity to productively multitask made shared ride and commuter rail more attractive but bus transit and driving alone less attractive. Results continue to be published using these data.

Other studies - most dealing with automobile use-looked specifically at the effects of attitudes and motivations related to travel experiences. In one study, the percentage of car commutes was associated with a person's symbolic and affective but not instrumental motivations (Steg, 2005). Another study positively associated "car pride" with for measures of car use (Zhao \& Zhao, 2015). A meta-analysis concluded that car use attitudes were moderately to strongly correlated with both driving and driving intentions (Gardner \& Abraham, 2008). In a stated preference study, people placing greater importance on travel happiness were more likely to choose to drive than to ride transit (Duarte et al., 2010). Modal perceptions of comfort and protransit and proactive travel attitudes have also been positively associated with commute mode choice (Malokin et al., 2015).

A 1998 survey of around 1,900 residents (including 1,300 commuters) near San Francisco, California, provided substantial empirical information about the PUT concept. The survey investigated desired travel amounts, excess travel indicators, mode- and purpose-specific travel liking, and travel-related attitudes (Mokhtarian, Salomon, \& Redmond, 2001)—what are classified as travel experiences or general PUT. These PUT measures were positively associated with self-reported travel amounts (distance, duration, or frequency), as expected (Mokhtarian et al., 2001; Schwanen \& Mokhtarian, 2003; Schwanen \& Mokhtarian, 2005). The study culminated in structural equation models (Ory, 2007; Ory \& Mokhtarian, 2007; Ory \& Mokhtarian, 2009), which tended to find a positive 
effect of travel liking on travel amounts for discretionary purposes but a negative effect for mandatory purposes. As Ory and Mokhtarian (2009) note, this suggests two directions of causality: Liking discretionary travel can lead to more of it, while being forced to travel for mandatory activities decreases travel liking (p. 38). These results could also indicate that the (purpose-specific, not trip-specific) travel liking questions captured a halo effect from destination enjoyment or other response bias.

Overall, it appears that PUT may indeed have a modest impact on travel behavior, however those effects are likely context dependent, varying with travel mode, purpose, and/or other trip characteristics. Multitasking and a propensity or ability to multitask may affect mode choice, but results remain limited. Attitudes related to travel experience factors appear to influence at least driving behaviors. While general measures of travel liking have been associated with trip-making or distances traveled, questions remain about directions of causality and self-selection (De Vos \& Witlox, 2016). More research on the travel behavior effects of a PUT is needed, particularly using trip-level measures and investigating mode choices.

\subsection{Implications of the positive utility of travel concept}

\subsubsection{For research}

This review makes several contributions to travel behavior research. First, it provides a stronger conceptualization of PUT, building upon and updating past work to distinguish between destination activities, travel activities, and travel experiences and their subcomponents. Future studies and surveys can use this review to structure PUT data 
collection and to avoid missing a particular component. Notably, it also highlights important connections to concepts like utility, motivation, and subjective well-being.

Second, the review of methods and evidence offers guidance for studies to more directly investigate the PUT concept. In the social science approach to causality, one must provide evidence of not just association and time precedence but also nonspuriousness (Singleton \& Strait, 2005). Thus, if the goal is to measure a PUT and its influence on travel behavior, researchers should use methods that provide evidence consistent with the PUT concept but that also eliminate alternative hypotheses by directly addressing its underlying mechanisms. This rules out many aggregate observational general PUT methods in favor of direct questioning about desired travel amounts, excess travel indicators, and the teleportation test. Even better would be to separately ask about specific PUT components: travel-based multitasking; and subjective well-being and satisfaction with travel, affective responses and noninstrumental reasons for travel, and travel liking. Often, careful wording of questions and items (Mokhtarian, Salomon, \& Singer, 2015; Russell \& Mokhtarian, 2015) can better elicit useful responses and reduce potential confounding influences.

Third, the summaries of potential determinants of a PUT and its effects on travel behavior highlight research gaps to be addressed. Few personal characteristics have been consistently associated with a PUT; future work should consider broader life stage, lifestyle, or cultural influences as well as more detailed attitudes. Overall, more work is needed to identify potential determinants, especially those that can be more easily and reliably measured than the detailed and complex direct PUT measures. Notably, few studies have examined the effects of a PUT on travel behaviors. There is a need for considerable additional research, particularly looking at both travel activity and travel 
experience components, measuring mode-specific and especially trip-specific PUT, and investigating impacts on mode choices.

Finally, this review helps to acknowledge the existence of a PUT. Armed with evidence that the PUT concept is a real phenomenon, researchers should consider examining it as part of travel behavior studies. Better measurement of the positive aspects of traveling may also help to improve understandings and estimates of other behavioral influences: values of travel time, perceptions of safety and security, residential location choice, habits, and (especially) attitudes. Other scholars are encouraged to use this literature review to design future studies that increase the field's collective knowledge of the PUT concept.

\subsubsection{For planning, policy, and the future}

This review concludes by discussing potential implications of the PUT idea for transportation planning, policymaking, and the future. Many consequences for transportation planning involve travel demand analysis. PUT measures are rarely included in travel demand models, so their omission (like that of any omitted variable) has been shown to bias parameter estimates in a simulation study (Singleton \& Clifton, 2015). This bias results from endogeneity issues (Fernández-Antolín et al., 2016), specifically correlations between observed variables and the error term due to unobserved factors related to the PUT concept. For example, consider mode choice. A PUT correlated with travel time may falsely attenuate (if positively correlated) or magnify (if negatively correlated) the magnitude of the estimated travel time coefficient. This parameter is used to calculate VTTS, an important input to cost-benefit analyses of major transportation 
projects (Mackie, Jara-Díaz, \& Fowkes, 2001). Biased values may yield incorrect estimates of mode/route shifts and (more importantly) of the user benefits of mobility-enhancing projects. PUT effects may also manifest in spuriously significant traveler characteristics or mode-specific constants. In the long run, if researchers can satisfactorily measure, forecast, and translate PUT into a predictive model (a big ask), planning tools might be better able to evaluate a wider array of transportation projects, programs, and policies: e.g., walking and bicycling demand, which may be influenced by nonutilitarian aspects (especially exercise benefits).

Policy implications of PUT depend upon the direction and magnitude of its impact on travel behavior. While there is currently insufficient research to quantify magnitude, the PUT conceptualization in this chapter points towards clear directions of influence that could be useful for designing or evaluating transportation policies. For instance, consider interventions to reduce automobile use. Instead of increasing the disutility or generalized cost of driving, policies could increase the positive utility or benefits (through productivity or enjoyment) of nonauto modes. For public transit, WiFi and tables could encourage productive use of in-vehicle travel time, while comfortable seating or crowding reduction could increase pleasure. Protected bike lanes may make bicycling feel safer and more enjoyable; more attractive sidewalks and human-scale streetscapes could do the same for walking. Other interventions could target active transportation: wider bike lanes to support side-by-side conversations (McIlvenny, 2014); countdown timers at bicycle traffic signals to allow quick phone use; "smartphone walking lanes" (Kaplan, 2015) for productive walking; or popular exploratory games like Pokémon Go. These policies may not be perfect 
or even desirable, but they follow logically from an understanding of the PUT idea and its potential behavioral effects.

While relevant today, the PUT concept will likely play a more central role in personal transportation choices in the future. Advances in internet- and GPS-based ICTs like smartphones and in-vehicle "infotainment" systems have already made traveling more enjoyable and productive. The productivity benefits afforded by the development of semiand fully-autonomous vehicles (AVs) has the potential to dramatically affect travel behaviors (Zmud, Sener, \& Wagner, 2016). Some physical and mental resources currently dedicated to the driving task could be reallocated to more active forms of travel-based multitasking. As vehicle designs continue to emphasize passenger comfort and entertainment, self-driving cars may also generate more positive affect from the travel experience. Recent studies have modeled potential impacts of AV-enabled productivity increases (Childress, Nichols, Charlton, \& Coe, 2015; Kim, Rousseau, Freedman, \& Nicholson, 2015; Levin \& Boyles, 2015; Malokin et al., 2015; Pawlak, Polak, \& Sivakumar, 2015; van den Berg \& Verhoef, 2015), presuming a reduced disutility of travel time; however, most assumptions are still speculative. Analyzing the effects of a PUT today could help begin to quantify some of the potential implications of ubiquitous vehicle automation.

This discussion raises other questions related to the PUT concept and AVs. What happens when "driving" feels much like being at work or home? Will traveling become a secondary activity, where travel is the setting for activity participation? Will AVs poach transit riders, or will they provide feeder service to enhance high-capacity transit? Who will purchase or use AVs: those who seek ultraproductive travel (Lyons \& Urry, 2005), or 
those who value leisure time? How will people with symbolic motivations for car use (freedom, exploration, power) travel when cars are not to be driven? Will independenceseekers turn to other modes (like cycling) because they can control the speed, direction, route, and other aspects of vehicle operation? The answers to these questions are unclear, but they are rooted in the PUT notion. Studying the positive utility of travel could help us better anticipate and prepare ourselves and our transportation systems for an uncertain future. 


\section{Chapter 3 Data collection}

\subsection{Abstract}

The "positive utility of travel" (PUT) concept suggests that people may find benefit from traveling beyond reaching a destination: e.g., by making productive use of travel time, or by enjoying the experience of traveling itself. These aspects have seen increased attention in the travel behavior field, although empirical evidence about them and their potential effects on behaviors like mode choice remains limited. To remedy this gap, an online questionnaire survey was developed to measure attributes about both major aspects of the PUT concept - travel activities (or travel-based multitasking) and travel experiences (or subjective well-being)—with respect to commute mode choice. The survey instrument included questions on commuters' personal characteristics, their typical commutes, the things they did and felt while on a recent commute, and the things they would have done or felt if they had used a different mode for that trip.

Following a lengthy survey development process and a small pilot study, the Commuting Survey 2016 was administered to commuters in the Portland, OR, metropolitan area during October, November, and December 2016. Participants were recruited using several techniques: at workplaces, through email invitations to major employers and organizations of employers; via direct email targeted at downtown workers; and by handing out postcards to nonmotorized commuters in the field. Around 650 complete responses were received to the main Part I of the survey; another 475 people completed an optional Part II of the survey. The survey oversampled nonautomobile commuters, by design, in order to get a large enough sample to examine modal differences 
and sensitivities. Although the sample skewed towards higher-income workers, other socio-demographic characteristics were roughly reflective of their distribution in the local population.

The questionnaire succeeded in measuring many PUT-related attributes. Commuters reported doing a number of different activities while traveling, including listening to music, thinking, and viewing scenery. Most travelers also reported feeling alert, attentive, and calm, and considered their commutes to provide a buffer between home and work. Results also suggested important modal differences in aspects related to the PUT concept. People walking and bicycling reported having more useful commutes and liking them better. Detailed results are documented in other chapters. This chapter describes the data collection effort, including study motivations, the survey development process, the contents of the questionnaire, recruitment procedures, data processing and analyses tasks, and preliminary results.

\subsection{Introduction}

Why do people travel? Within the transportation research field, the derived-demand paradigm assumes that people travel to reach destinations where they can conduct activities. It also assumes that travel is a disutility to be minimized. In short, people travel to get places; travel is a means to an end. However, these assumptions may not be universally true. Sometimes, people may be motivated to travel for other reasons, and they may receive benefits from traveling itself. For instance, some may commute by train in order to sleep or get work done. Others may choose to walk or bicycle to get places while simultaneously exercising. These travelers are using their travel time productively for 
multiple activities. The experience of traveling can also provide benefits or be a primary objective: the commute as an escape or transition between work and home; driving a sports car to feel powerful or indicate social status; or riding public transit because of an environmental consciousness. In these cases, the travel experience can provide physical, emotional, or symbolic benefits.

The idea that travel can provide benefits and may be motivated by factors beyond reaching activity destinations is known in the travel behavior field as "the positive utility of travel" (PUT). Since this concept gained broader attention in the travel behavior research arena more than 15 years ago (Salomon \& Mokhtarian, 1998; Mokhtarian \& Salomon, 2001), many studies have examined various aspects of PUT, both empirically (e.g., Ory \& Mokhtarian, 2005; Diana, 2008; Malokin et al., 2015) and more conceptually (e.g., Hess et al., 2005; Mokhtarian, Salomon, \& Singer, 2015; Singleton \& Clifton, 2015). Despite this small but growing area of interest, studies conceive of PUT in different ways, use varying language when describing PUT, and ask an assortment of questions that may or may not provide convincing evidence of a PUT instance. Few empirical studies have looked in depth at all aspects of PUT simultaneously, and research is only beginning to investigate the impacts of PUT factors on travel behaviors like mode choice (see Chapter 2).

Against this backdrop, this study was developed to fill these conceptual and empirical gaps in travel behavior literature with respect to the PUT concept. Specifically, an online questionnaire survey was developed to measure PUT attributes with respect to commute mode choice in Portland, Oregon. The study and the survey were designed to address the following research questions: 
1. What is the positive utility of travel (PUT) concept?

a. Conceptually, how is the PUT idea defined and structured?

b. Empirically, how can a positive utility of travel be measured?

2. What are potential determinants of a PUT?

a. What personal and travel characteristics predict PUT measures?

3. How do positive utilities of travel affect travel behavior?

a. How do PUT attributes impact travel mode choice?

To answer these questions, the survey was structured as a questionnaire and travel diary (methods typically used to collect data on mode choice behavior) with additional detailed questions about the PUT concept. Travel activity aspects were measured by questions about the specific activities people engaged in while commuting (travel-based multitasking), and their thoughts about the overall usefulness of the time they spent traveling and doing those activities. Travel experience aspects were measured by a series of questions related to subjective well-being (SWB) in the travel domain, including the Satisfaction with Travel Scale (an existing validated instrument); other questions about emotions, feelings, and meaning derived from the act of commuting by particular modes; and an overall assessment of travel liking.

This report documents the data collection process for and preliminary results from a survey — called the Commuting Survey 2016 — designed to investigate these PUT issues and their potential effects on mode choice behavior. It proceeds as follows: First, the survey development process is described, including how key questions about travel activity and experience attributes were developed and their examination in a small pilot study. Next, the online questionnaire is described in detail, including the two parts to the survey, their 
primary question blocks, and a summary of the questions included in each block. (The full survey instrument is attached in the Appendix.) Participant recruitment and survey administration procedures are then described, followed by a summary of some of the steps involved with data processing and analysis. Finally, preliminary results of the surveyincluding key measures of the travel activities and travel experience aspects of the PUT concept—are presented.

\subsection{Survey development}

To measure the positive utility of travel concept, its determinants, and its linkages to commute mode choice, a questionnaire survey was developed. First, several other travel diary surveys — including the 2011 Oregon Household Activity Survey (OMSC, 2011)— were examined for traveler and trip characteristics that could be relevant for a mode choice study. Next, major studies that investigated PUT-related aspects - including two efforts led by Mokhtarian, one in 1998 (Curry, 2000; Mokhtarian \& Salomon, 2001; Mokhtarian, Salomon, \& Redmond, 2001; Redmond \& Mokhtarian, 2001) and another in 2011 (Neufeld \& Mokhtarian, 2012) — were examined, and the question and item wordings for relevant measures of both travel activity and travel experience attributes were extracted and considered. Finally, a literature review of the PUT concept and a critique of methods and evidence for PUT (Chapter 2) identified the most valuable means of measuring PUT attributes and some sources for borrowing specific questions. 


\subsubsection{PUT question/item selection}

To measure trip-specific travel-based multitasking - the primary contributor to the travel activity component of the PUT concept—a list of activities was developed. First, all activities that were included on a questionnaire of travel-based multitasking were pulled from numerous previous studies on multitasking (Circella et al., 2015; Ettema et al., 2012; Ettema \& Verschuren, 2007; Guo et al., 2015; Kenyon, 2006; Keseru et al., 2015; Lyons et al., 2007; Lyons et al., 2013; Malokin et al., 2015; Ohmori \& Harata, 2008; Russell et al., 2011; Timmermans \& van der Waerden, 2008; van der Waerden et al., 2009; Yosritzal, 2014; Zhang \& Timmermans, 2010). Next, these activities were grouped into broad categories (e.g., communicating, using media). Finally, from this long list, 23 of the most commonly used or reported activities were selected to represent a broad range of potential activities conducted during travel. See the Questionnaire section below or the Appendix for a full list of questions and items related to the travel activity component of PUT.

To measure trip-specific subjective well-being (SWB), the primary contributor to the travel experience component of the PUT concept, several types of questions were used: questions about the affective component of hedonic SWB (travel affect), questions about both affective and cognitive aspects of hedonic SWB, and questions about the symbolic and purposeful component of SWB (travel eudaimonia). An existing instrument, the Satisfaction with Travel Scale (STS) (Ettema et al., 2012; Smith, 2017), was used as a measure of the second aspect, overall hedonic SWB. The development of questions and items to represent the travel affect and eudaimonia components of SWB in the travel domain are described in the following paragraphs. 
Measures of travel affect were adapted from existing psychological instruments for affective SWB. An approach similar to that used by the Positive and Negative Affect Schedule (PANAS) was deemed to be most useful, since it is simple to administer and has been designed to work with shorter times scales like a particular activity (Watson, Clark, \& Tellegen, 1988). The PANAS asks respondents to "indicate to what extent" they felt each of a series of adjectives on a five-point Likert-type scale. Due to the length of the survey, the full 20-item PANAS was not used. Instead, the first block of travel affect questions was composed of the 10 items from the international short-form version (IPANAS-SF), which has been psychometrically validated (Thompson, 2007).

A multistage process was used to round out the second block of 10 travel affect items (see Chapter 5). First, a master list of about 120 adjectives or short phrases relating to affect, emotion, or mood was developed. The words were pulled from standard psychological affect scales (including PANAS and its variants), travel behavior literature that investigated travel affect (Anable \& Gatersleben, 2005; Diana, 2008; Ellaway et al., 2003; Handy et al., 2005; Gatersleben \& Uzzell, 2007; Loo et al., 2015; Milakis et al. , 2015; Mokhtarian, Salomon, \& Singer, 2015; Morris \& Guerra, 2015a; Rhee et al., 2013; Steg, 2005; Thomas \& Walker, 2015), and a few suggestions by the author. Next, about 100 of the most promising of these adjectives were included in a PANAS-type questionnaire about feelings during transportation that was administered to a small sample of acquaintances $(N=11)$ with a variety of ages and genders. Finally, the remaining 10 items were selected from this list based on three considerations: inclusion in another psychometric instrument (like the PANAS-X), frequent association with travel (from the literature and the small sample survey), and lack of overlap with constructs already in the 
I-PANAS-SF or the STS. The full 20-item list of travel affect measures is shown in the Appendix and the Questionnaire section below.

To measure the eudaimonic aspects of travel well-being, new questions and items were created. Existing instruments for measuring eudaimonic SWB were considered, but adaptation to the travel domain was deemed infeasible (see Chapter 5). Instead, a multistage process was conducted, similar to what was done to select travel affect items. First, a master list of about 75 words or short phrases were pulled from existing psychological scales (e.g., Diener et al., 2010), travel behavior literature investigating travel eudaimonia (Anable \& Gatersleben, 2005; Diana, 2008; Ellaway et al., 2003; Handy et al., 2005; Loo et al., 2015; Mokhtarian, Salomon, \& Singer, 2015; Ory \& Mokhtarian, 2005; Rhee et al., 2013; Salomon \& Mokhtarian, 1998; Steg, 2005), and some author suggestions. Next, about 70 of these words were included in the same small-sample $(N=$ 11) questionnaire. Finally, 22 words/phrases were selected based on: frequent association with travel (from the literature and the survey); and coverage of a number of concepts identified in a literature review of the PUT concept (Chapter 2). Each of the 22 travel eudaimonia items were grouped into one of three question blocks, reflecting potential motivations for travel: to "fulfill your desire for," "express," or "improve" something. The Appendix and the Questionnaire section below contains details on the three blocks of items used to measure travel eudaimonia.

\subsubsection{Pilot}

Before deploying the survey for final data collection, a pilot deployment was undertaken. This piloting involved two steps: first, administration of the initial survey to a 
small sample $(N=6)$ of willing participants; and second, a focus group and debrief session with the pilot survey respondents. The survey instrument, an online questionnaire designed through Qualtrics, was administered to six employees of a small technology company located in the central city of Portland, Oregon, in September 2016. After all participants completed the survey, but within 24 hours, respondents met with the author for a debriefing session, in which they shared their thoughts about the survey, any confusing sections, and suggestions for improvement. Participants were compensated for their time with a free lunch.

Several changes were made to the survey as a direct result of the piloting and participants' feedback. Most significantly, a few respondents took far longer than expected to complete the survey, so several less-essential questions were moved to an optional Part II of the survey, and estimated completion times were revised. Other less-essential and confusing questions were removed entirely. The preliminary version of the survey asked for responses to travel eudaimonia questions on a five-point Likert-type scale, à la PANAS, however qualitative responses suggested this level of distinction was a difficult task that took too much time to complete. To reduce respondent burden, the response scale for travel eudaimonia was changed to a yes/no checkbox, and the text "at least a little" was added to the question to make it somewhat comparable to the first level in the PANAS scale. Finally, the wordings and layouts of several questions and items throughout the survey were revised to improve interpretability and ease of understanding in response to feedback from the pilot survey participants. 


\subsection{Questionnaire}

The primary data collection effort involved the use of an online questionnaire, composed of several blocks of questions. The questionnaire was designed and administered through Qualtrics. Although a single long survey was considered, pilot testing suggested respondent fatigue from answering online questions for an hour or more. Instead, it was decided to split the survey into two parts: a main Part I that would take a more reasonable 30 to 40 minutes to complete, and an optional Part II that would take an additional 15 to 20 minutes to complete. The components of the questionnaire are described in the following sections. The Appendix contains the survey instruments for Parts I and II, including all question and item wordings.

\subsubsection{Part I}

Part I of the Commuting Survey 2016 began with a descriptive introduction page and indication of consent. Two eligibility questions further restricted participants to those who were adults (aged 18+) and who commuted to a job outside the home at least weekly.

\subsubsection{Your personal and transportation characteristics}

The first group of questions asked for basic socio-demographic characteristics and information about a respondent's transportation availability and experience. Many of these questions were adapted from two recent Portland-area surveys: the Oregon Household Activity Survey (OHAS) (OMSC, 2011), and a survey about commute SWB conducted by Smith (2013). This section took about 5 minutes to complete. 
Personal characteristics included:

- Age, in categories;

- Racial and ethnic identity, adapted from questions being tested for the 2020 U.S. Census (Cohn, 2015);

- Gender identity, including nonbinary options, adapted from the second question in the two-step recommendations of the GenIUSS report (GenIUSS, 2014);

- Education level, adapted from a question on the OHAS;

- Student status, also adapted from an OHAS question;

- Household size and characteristics (relationship, student status, worker status, age) of other household members, adapted from questions on Smith's survey; and

- Household income, also adapted from a question on OHAS.

Transportation characteristics included:

- Disability status, mobility limitations, and mobility skills, adapted from a question on the Smith survey and questions on the American Community Survey (US Census Bureau, 2016);

- Driver license holding, adapted from an OHAS question;

- Household vehicle availability, including both automobiles and bicycles, adapted from a question in Smith;

- Transit pass holding, including whether this was through an employer;

- Membership in a particular vehicle-sharing (car-share and bike-share) service; 
- Use of means of transportation other than personally-owned automobiles, including public transit modes, mobility as a service (Uber, Lyft, taxis), and vehicle-sharing programs; and

- Use of various travel modes within the past week.

\subsubsection{Your home, your job, and your typical commute}

The second section contained questions about a respondent's home, their job, and characteristics of their typical commute. It also included questions about the PUT concept as relating to typical commutes, including satisfaction with travel time and thoughts about commuting scenarios. This section took approximately 10 minutes to complete.

Questions about a respondent's home or place of residence included:

- Housing type, adapted from questions in OHAS (OMSC, 2011) and the ACS (US Census Bureau, 2016);

- Housing tenure or homeownership status, adapted from OHAS;

- Duration of time living in current home, also adapted from OHAS; and

- Home location, specified as the nearest intersection.

For workplaces and jobs, the following questions were asked:

- Job occupation (as a free text response) and self-employment status, adapted from an OHAS question;

- Days and hours worked per week;

- Work schedule flexibility; and

- Job location, specified as the nearest intersection. 
The subsection on typical commutes had instructions to consider a commute on a normal or average day at this time of the year. Questions included:

- Travel mode, either the mode used for a typical commute or (if multiple modes were typically used) the one used for the longest duration, from among the following list:

○ Walking;

○ Bicycling;

○ Automobile, driver;

○ Automobile, passenger; and

Public transit;

- Travel mode follow-up questions about the type of automobile or transit vehicle, as necessary;

- Commute distance in miles; and

- Commute durations in minutes, for both home to work and work to home commutes.

Next were questions about various commuting scenarios. These were designed as a simple stated choice experiment that pivoted off a respondent's typical allocation of time to work and the commute. Four options were developed, where the time spent working or the time spent commuting either increases or decreases by 10 minutes, compared to normal work responsibilities and a typical commute. Pay and travel costs were held fixed. Thus, respondents were instructed to choose from among two marginal changes in work and commute time for the full enumeration of six choice scenarios (question order was fixed but item order was randomized): 
- Work time increases vs. Work time decreases;

- Commute travel time increases vs. Commute travel time decreases;

- Work time increases vs. Commute travel time increases;

- Work time decreases vs. Commute travel time decreases;

- Work time increases vs. Commute travel time decreases; and

- Work time decreases vs. Commute travel time increases.

The final subsection about the PUT concept in general contained preliminary text that instructed respondents to consider the things they might like or dislike about commuting, with examples. This introduction was adapted from recommendations by Russell and Mokhtarian (2015). Following this preparation, questions were asked about:

- Overall satisfaction with typical commutes and satisfaction with commute travel time specifically, adapted from questions about commute satisfaction (Abou-Zeid \& Ben-Akiva, 2011; Mao et al., 2015; Milakis et al., 2015; Rasouli \& Timmermans, 2014a; St-Louis et al., 2014; Susilo et al., 2017; Wachs et al., 1993);

- Satisfaction with hypothetical commute times $(0,10,20,30$, and 45 minutes, and 1 and $2+$ hours), adapted from some of the same previous studies (Milakis et al., 2015; Susilo et al., 2017; Young \& Morris, 1981);

- Ideal commute travel time, adapted from questions in other studies (Milakis et al., 2015; O'Fallon \& Wallis, 2012; Páez \& Whalen, 2010; Redmond \& Mokhtarian, 2001; Russell, 2012; Watts \& Urry, 2008); and

- The teleportation test, in which people are given the choice to teleport or to spend some (nonzero) time commuting. This question was adapted from 
a few recent studies (Diana, 2008; Handy et al., 2005; Jain \& Lyons, 2008;

Mokhtarian \& Salomon, 2001; O'Fallon \& Wallis, 2012; Russell, 2012;

Watts \& Urry, 2008), particularly the recommended wording and followup questions from a recent review on the subject (Russell \& Mokhtarian, 2015).

\subsubsection{Your most recent commute trip, including things you did and things you felt and experienced while commuting}

The heart of the survey, and the section that took the most time, included detailed questions about each respondent's most recent commute trip from home to work, including PUT-specific questions about the things they did (travel activities) and the things they felt and experienced (travel experiences) while commuting. Depending on the speed at which people answered these questions, this section took around 10 to 15 minutes to complete.

The first questions gathered basic information about the most recent trip from home to work:

- When it occurred, including how many days ago, the day of the week, and the precise time that a respondent left home and subsequently arrived at work;

- Primary commute mode, or (if multiple) the one used for the longest duration;

- Travel mode follow-up questions about the type of automobile or transit vehicle, as necessary; 
- Questions about parking facility type and location, specified to the nearest intersection, if driving an automobile;

- A follow-up question about other modes used during the commute, such as to getting to or from public transit or a parking space;

- Monetary cost, specified as direct costs paid that day (to include parking, transit, or ride-hire fares but not gas, maintenance, or monthly parking/transit costs);

- The number of other people traveling in the same party; and

- The number of intermediate stops made between home and work, including detailed information about each:

- The stop location, specified as the nearest intersection;

- The purpose(s) for the stop, from among 12 choices adapted from OHAS trip purposes (OMSC, 2011); and

- The duration of the stop, in minutes.

For the chosen commute mode, questions then asked about travel activity aspects of PUT:

- Activity participation, including the option to select multiple activities from a list of 23 activities (chosen as described in the Survey development section above), including an "other" option:

○ Talking face-to-face with people you know;

- Talking face-to-face with strangers;

- Talking on the phone;

- Texting, emailing, or other messaging; 
- Reading print (newspaper, book, etc.);

○ Reading electronically (e-book, website, etc.);

- Writing or editing paper documents;

- Writing or editing electronic documents;

- Listening to music, radio, or other audio;

- Watching movie, TV, or other video;

- Using social websites or apps (Facebook, Twitter, LinkedIn, Tumblr, Instagram, etc.);

○ Playing game (Pokémon Go, puzzle, etc.);

○ Eating food; drinking beverage;

- Smoking or vaping;

○ Personal grooming (shaving, makeup, etc.);

- Caring for children or pets (dog walking, etc.);

○ Singing; dancing;

○ Exercising or being physically active;

- Planning or navigating this trip;

- Viewing scenery; watching people;

○ Thinking or daydreaming;

○ Sleeping or snoozing; and

$\circ \quad$ Doing nothing;

- Activity duration, specified as the approximate percentage of travel time spent on the activities selected in the previous question; and 
- Travel usefulness, or the value of the time spent doing these activities on a five-point Likert-type scale (Mostly wasted; Somewhat wasted; Neither wasted nor useful; Somewhat useful; Mostly useful), as adapted from questions used in other studies about the value, worth, or usefulness of a trip (Circella et al., 2015; Frei et al., 2015; Lyons et al., 2007; Lyons et al., 2013).

The next group of questions asked about travel experience aspects of PUT:

- Travel affect, or feelings and emotions, measured by responses to items assessed on a five-point Likert-type scale (Very slightly or not at all; $A$ little; Moderately; Quite a bit; Extremely) and grouped into two blocks:

o First, 10 items comprising the I-PANAS-SF (Thompson, 2007): Upset; Hostile; Alert; Ashamed; Inspired; Nervous; Determined; Attentive; Afraid; and Active; and

- Second, 10 items selected using the process described earlier in the Survey development section: Excited; Strong; Vulnerable; Proud; Angry; Bold; Frustrated; Timid; Calm; and Stressed;

- Travel sensations, including checkboxes as to whether respondents felt the following or none of the above: Hot; Cold; Wet; Sore; Dirty; and/or Sweaty;

- The Satisfaction with Travel Scale (Ettema et al., 2011), as translated into English and modified upon others' recommendations (De Vos et al., 2015; Smith, 2017) to more closely fit the intended constructs, measured using 
responses on a seven-point semantic differential scale to nine items of paired statements:

- I was very tense ... I was very relaxed;

- I was very bored ... I was very enthusiastic;

- I was very sad ... I was very happy;

- I was very tired ... I was very energized;

- I was very distressed ... I was very content;

- My trip went poorly ... My trip went smoothly;

- My trip was displeasing ... My trip was enjoyable;

- I was worried I wouldn't arrive on time ... I was confident I would arrive on time; and

- My trip was the worst I can imagine ... My trip was the best I can imagine;

- Travel eudaimonia, measured by checkbox responses to a number of items selected using the process described earlier in the Survey development section (including a "none of the above" option) and grouped into three blocks:

- Things for which commuting fulfilled a desire: Variety; Control; Adventure; Companionship; Freedom; Privacy; Safety; Comfort; Stress relief; A routine; A challenge; A buffer between home and work; and Membership in a group or class; 
○ Things for which commuting expressed: Independence; Social status; Self-identity; Courage; Mastery of a skill; and Environmental values; and

$\bigcirc$ Things that commuting improved: Self-confidence; Mental health; and Physical health; and

- Travel liking, or the overall enjoyment of the commute, measured on a five-point Likert-type scale (Strongly disliked; Somewhat disliked; Neither liked nor disliked; Somewhat liked; Strongly liked), as adapted from travel liking or enjoyment questions used in other studies (Ory \& Mokhtarian, 2005; Turcotte, 2006; Gatersleben \& Uzzell, 2007; Mokhtarian, Papon et al., 2015).

A final subsection asked about retrospectively about commute expectations, including:

- An overall comparison between the commute experience and expectations;

- Any changes that would have been made with more knowledge; and

- Anything special, different, or unexpected that occurred.

3.4.1.4 Other modes you could have used, including things you would have done and things you would have felt and experienced while commuting

This section of the survey was intended to capture similar information about PUTrelated attributes for alternative travel modes that were considered but not chosen. It included questions about other modes and, for each mode selected, very similar PUTspecific questions about the things a respondent would have done (travel activities) and the 
things they would have felt and experienced (travel experiences) if they would have commuted via that mode. Depending on the number of alternative modes selected, this section took approximately 5 to 15 minutes to complete.

First were questions about other commuting options and modes, assuming a respondent's chosen mode was not available, including:

- Whether commuting from home would have been preferred;

- What other modes (at least one) were considered;

- Travel mode follow-up questions about the type of automobile or transit vehicle, as necessary;

- A follow-up question about other modes used during the commute, such as to getting to or from public transit or a parking space;

- Rankings for multiple alternative modes; and

- Reasons (free text) for not considering other commute modes.

For each alternative mode selected, the same sequence of questions about PUTrelated aspects were asked, with minimal changes in tense (e.g., "would have"). Travel activity questions - activity participation, activity duration, and travel usefulness - were identical. Travel experience questions - travel affect, travel sensations, the Satisfaction with Travel Scale, travel eudaimonia, and travel liking-were nearly identical, except travel affect questions that had been measured on a five-point Likert-type scale were instead measured with checkboxes to ease response burdens.

The survey concluded with a few wrap-up and survey administration questions. First, respondents were asked if they wanted to enter the drawing and, if so, for an email address at which to contact them. Next, a brief description of the optional Part II survey 
was provided, and all respondents were given the option to complete it either immediately through redirection after submitting the Part I survey, or in the future by copying or receiving an email with a link to the Part II survey. Finally, all respondents were given the opportunity to provide additional comments in a text box before finishing the survey.

\subsubsection{Part II (optional)}

Part II of the Commuting Survey 2016 also began with a descriptive introduction page and indication of consent. No eligibility questions were necessary because only those who had completed Part I (and thus indicated their eligibility) had access to Part II. Instead, respondents were instructed to provide their email address in order to link their otherwise anonymous responses in Part II to their responses in Part I.

\subsubsection{How you get around using different means of transportation}

The first group of questions asked about the use of different means or modes of transportation. This section took about 5 minutes to complete. It included questions about:

- Mode use frequency for each of the five primary modes (walking, bicycling, auto driver, auto passenger, public transit) at this time of year, as adapted from a question on Smith's (2013) survey;

- Relative desired mobility, specifically whether respondents wanted to use each mode more or less than they currently did, as adapted from questions used in other studies (Curry, 2000; Handy et al., 2005; O'Fallon \& Wallis, 2012; Redmond, 2000; Russell, 2012); and

- For each mode used at least once a week: 
- Trip purpose(s) that the mode is used to accomplish, from among the same list of 12 described earlier; and

○ Modal perceptions, measured using a five-point semantic differential scale for 11 items:

- Slow ... Fast;

- Expensive ... Affordable;

- Inconvenient ... Convenient;

- Unpredictable ... Reliable;

- Risky ... Safe (from traffic collisions and injuries);

- Vulnerable ... Secure (from crime or violence);

- Unhealthy for me ... Healthy for me;

- Harms the environment ... Helps the environment;

- A waste of time ... A good use of time;

- Uncomfortable ... Comfortable; and

- Boring ... Fun.

3.4.2.2 Your thoughts about various topics, including multitasking, satisfaction, and attitudes

This next section asked about respondents' general thoughts on a number of topics that could be relevant for transportation or understanding travel behavior, including multitasking and perceptions of time, feelings and satisfaction with life and a job, and attitudes about technology, transportation, the environment, and health. This section took approximately 10 to 15 minutes to complete. Most questions were answered on a standard 
five-point Likert-type scale (Strongly disagree; Somewhat disagree; Neither agree nor disagree; Somewhat agree; Strongly agree).

The first group of questions were about attitudes/personalities related to travel activity aspects of PUT, including:

- Polychronicity, the preference for multitasking, as measured by the 14item Multitasking Preference Inventory (MPI) (Poposki \& Oswald, 2010); and

- Time perceptions, including 13 items related to time use, on-time behavior, and leisure time;

The next group of questions were about subjective well-being (SWB) and attitudes/personalities related to travel experience aspects of PUT, including:

- Feelings about life in general (positive and negative affect within hedonic SWB), as measured by the 10 -item International Positive and Negative Affect Schedule Short-form (I-PANAS-SF) (Thompson, 2007) assessed on the same five-point scale used to measure travel affect;

- Overall life satisfaction (both the cognitive aspects of hedonic SWB and the eudaimonic aspects of SWB), as measured by the five-item Satisfaction with Life Scale (SWLS) (Diener, Emmons, Larson, \& Griffin, 1985) and the eight-item Flourishing Scale (FS) (Diener, Lucas, Schimmack, \& Helliwell, 2009); and

- Job satisfaction (affective aspects), as measured by the four-item (plus 3 distracter items) Brief Index of Affective Job Satisfaction (BIAJS) (Thompson \& Phua, 2012); 
The final group of questions elicited general attitudes towards the following:

- Technology, as measured by the 12-item attitude section of the Media and Technology Usage and Attitudes Scale (MTUAS) (Rosen, Whaling, Carrier, Cheever, \& Rokkum, 2013);

- Transportation policies, including willingness to pay for different transportation investments and willingness to adopt sustainable behaviors, as borrowed and adapted from a number of different sources (AS \& MRSS, 2013; Mokhtarian et al., 2001);

- The environment, as borrowed and adapted from questions about environmental concern on the International Social Survey Programme (ISSP), administered in 1993, 2000, and 2010 (Franzen \& Meyer, 2010; Franzen \& Vogl, 2013); and

- Health and physical activity, as adapted from the health consciousness and health beliefs sections of the HealthStyles survey (Dutta-Bergman, 2004).

\subsubsection{Your physical activity levels}

The final section asked questions about respondents' physical activity levels as part of a job, transportation, and leisure time. These questions were borrowed from the Global Physical Activity Questionnaire (GPAQ) (WHO, 2017), as asked on the Trail Modeling and Assessment Platform (T-MAP) trail user survey (RTC, 2014). This section took about five minutes to complete.

For physical activity as part of a job or home chores, the survey asked for the number of days per week and the average amount of time in minutes on each of those days 
that a respondent engaged in vigorous intensity and moderate intensity physical activity. The same questions were also asked for physical activity as part of indoor or outdoor leisure activities, including sports and recreation. Respondents were then asked to report the number of days per week and the average amount of time in minutes on each of those days spent walking and bicycling for transportation purposes. A final question asked for the average time spent sitting or reclining while awake on a typical day.

\subsection{Recruitment and administration}

The target population for the survey included commuters in the Portland, Oregon, region, specifically adults who commuted to work outside the home at least once a week. To reach this working population, it was determined that contacting potential participants at their place of work would be most productive and avoid confusion if the survey reached a nonworking population. (For this reason, a random address-based sampling strategy was not considered.) Several specific recruitment strategies were adopted, as summarized in Table 3.1 and described in the following paragraphs. 


\section{Table 3.1 Summary of recruitment methods}

\begin{tabular}{llll}
\hline Method & Description & Date $(s)$ & Location(s) \\
\hline $\begin{array}{l}\text { Email recruitment } \\
\text { via employers \& } \\
\text { other organizations }\end{array}$ & $\begin{array}{l}\text { Contact transportation management } \\
\text { associations, chambers of commerce, } \\
\text { major employers; request to forward } \\
\text { email invitation \& survey link to their } \\
\text { members and/or employees. }\end{array}$ & $\begin{array}{l}\text { October 18 } \\
\text { November } \\
14,2016\end{array}$ & $\begin{array}{l}\text { Portland, OR, metropolitan } \\
\text { area }\end{array}$ \\
$\begin{array}{l}\text { Email recruitment } \\
\text { via direct email }\end{array}$ & $\begin{array}{l}\text { Contact people on City of Portland } \\
\text { downtown SmartTrips email list with } \\
\text { survey link. }\end{array}$ & $\begin{array}{l}\text { November } \\
1,2016\end{array}$ & Portland, OR, downtown \\
$\begin{array}{l}\text { Email recruitment } \\
\text { via neighborhoods }\end{array}$ & $\begin{array}{l}\text { Contact neighborhood associations; } \\
\text { request to forward email invitation \& } \\
\text { survey link to their members. }\end{array}$ & $\begin{array}{l}\text { November } \\
14,2016\end{array}$ & $\begin{array}{l}\text { Portland, OR, downtown, } \\
\text { central city }\end{array}$ \\
$\begin{array}{l}\text { Direct recruitment } \\
\text { via postcard } \\
\text { handout }\end{array}$ & $\begin{array}{l}\text { Hand out postcards with survey link } \\
\text { to passersby on bike and on foot. }\end{array}$ & $\begin{array}{l}\text { December } \\
13-14,\end{array}$ & $\begin{array}{l}\text { SW 1st Ave. \& SW Main St.; } \\
\text { SW Moody Ave. \& Tilikum }\end{array}$ \\
\hline
\end{tabular}

The primary means of participant recruitment involved contacting organizations of businesses and companies known as transportation management associations (TMAs). These TMAs are usually membership-based nonprofit organizations that offer programs for companies to employ transportation demand management (TDM) strategies: parking management, transit pass discounts, rideshare matching, commuter benefits, trip planning, etc. (VPTI, 2017). Staff at the five Portland-area TMAs-the Westside Transportation Alliance (WTA), Swan Island Business Association (SIBA), South Waterfront Community Relations (SWCR), Explore Washington Park, and Go Lloyd — were contacted via email with information regarding the survey and with a request to forward an invitation to their member businesses. The email also invited these employers to then share the survey invitation with their employees, the ultimate desired participants. All TMAs agreed to forward the survey invitation to their members via email or by including a link in a newsletter. A sample email invitation is shown in Figure 3.1. 


\section{Commuting Survey 2016}

Patrick Singleton <patrick.singleton@pdx.edu>

To:

\section{Commuting Survey 2016}

You are invited to participate in a Portland State University research survey about your commuting experiences. The information you provide will be analyzed to better understand transportation and commuting behaviors. The survey includes questions about:

- Your personal and transportation characteristics

- Your home, your job, and your typical commute

- Your most recent commute trip, including things you did and things you felt and experienced while commuting

The survey will take about 30 minutes to complete. If you complete the survey, you will have the opportunity to enter a drawing to win one of ten $\$ 100$ Visa gift cards.

Click here to take the survey!

https://sites.google.com/a/pdx.edu/commuting-survey-2016/

Your responses will be treated confidentially and will not be shared with your employer. Please complete this survey by December 16, 2016.

This study is being conducted by Patrick Singleton and Dr. Kelly Clifton, from the Department of Civil \& Environmental Engineering at Portland State University. The research is part of a doctoral dissertation, with funding from the National Institute for Transportation and Communities, a program of the Transportation Research and Education Center for Portland State University. If you have any questions about the study, please email tstudy@pdx.edu, call 503-893-9677, or write to: Portland State University, Civil \& Environmental Engineering, PO Box 751 - CEE, Portland, OR 97207-0751.

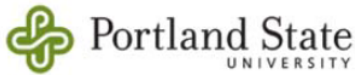

\section{Figure 3.1 Sample recruitment email for the Commuting Survey 2016}

In order to reach a wider cross-section of workers throughout the region, survey invitation emails were also sent to other established non-TMA organizations, including 13 chambers of commerce in the City of Portland and surrounding municipalities. Several of these organizations put a link to the survey in online newsletters and email communications. Some of the largest employers in the region were also contacted directly with a request to send a survey invitation to their employees.

A second and rather successful participant recruitment strategy utilized an existing email list maintained by the City of Portland; the same list was used for recruitment by 
Smith (2013). A survey invitation email was sent to approximately 3,700 email addresses of people who had participated in the City's SmartTrips programs (Dill \& Mohr, 2010) for downtown commuters between 2007 and 2009. While many people on the list likely no longer worked or worked downtown or in Portland, this effort did generate a large influx of participants during early November.

After the survey had been live for a few weeks, it became clear that more responses for walking (and a few more for bicycling) would be needed. Therefore, additional recruitment efforts were taken to target these nonmotorized commuters. First, 12 neighborhood associations located in or adjacent to Portland's central city were contacted via email with a similar request as was sent to the TMAs. Several neighborhoods sent the survey announcement to their membership lists or posted it to online sites like Facebook or Townsquared.

Second, a final push was made to gather nonmotorized participants in the final weeks of the survey. This effort involved intercepting mostly walking and some bicycling commuters at a few targeted locations in the central city of Portland as they made their way to work. Potential participants were greeted with a quick phrase (e.g., "Take a survey about your commute!") and handed a postcard containing basic information and a link to the survey. Field recruitment took place during cold weather from 7:30 to 8:30 AM, at SW 1st Avenue \& SW Main Street on Tuesday 13 December and at SW Moody Avenue \& Tilikum Crossing on Wednesday 14 December. On the first day, about 50\% of people approached accepted a postcard, and 74 postcards were handed out, two thirds to people walking and one third to people bicycling. On the second day, about $70 \%$ of people approached accepted a postcard, and 92 postcards were handed out, almost all to people walking. 
All participant recruitment methods involved the use of a URL that linked to the project website. On this website, the project goals were briefly described, and a link to the Qualtrics survey was highlighted. By directing potential participants to the project website first, this ensured that all links would still work even if the survey link itself might need to change due to edits or errors. Figure 3.2 shows a screenshot of the project website. Responses to the commuting survey were accepted between mid-October and midDecember 2016.

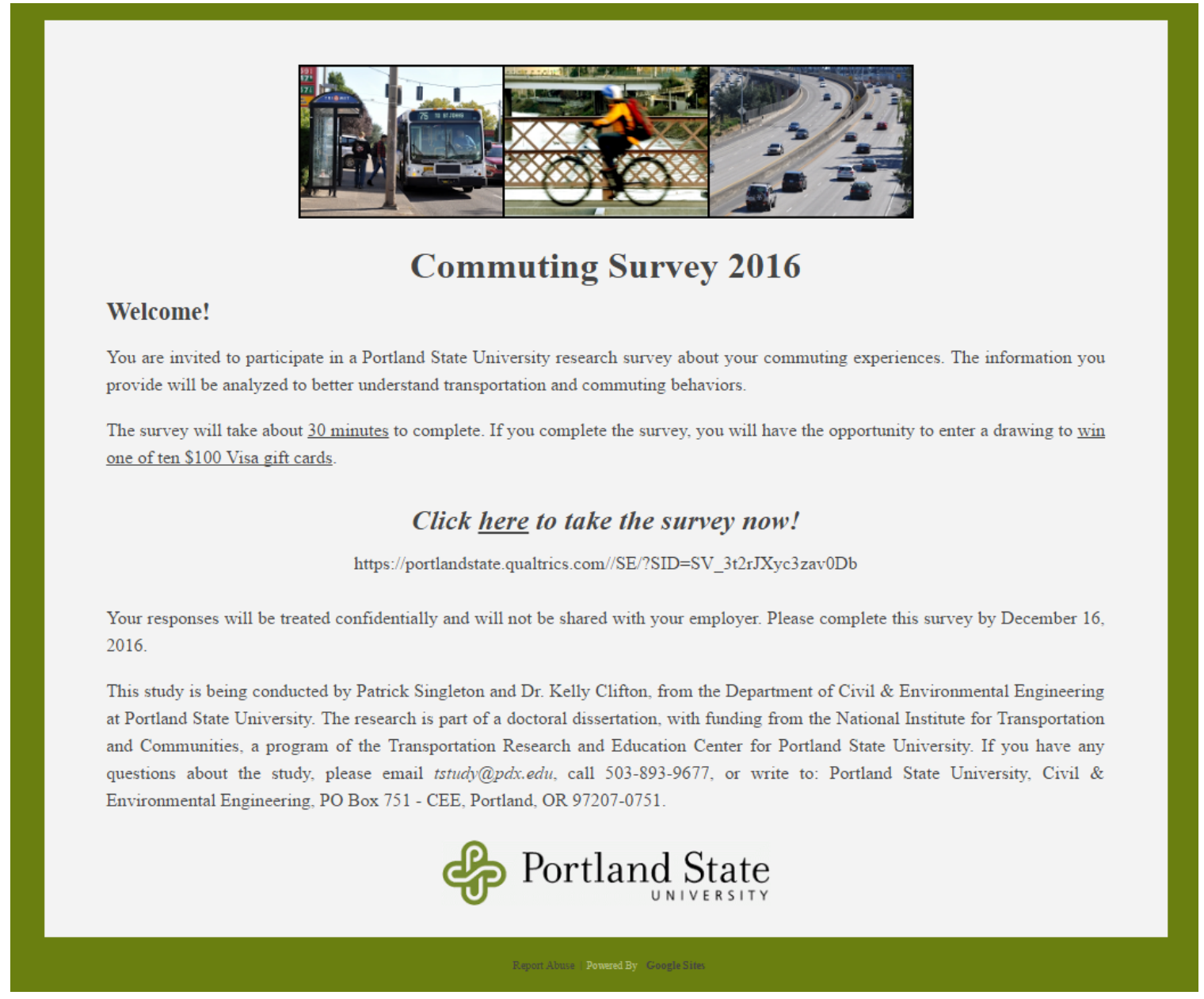

Figure 3.2 Project website for the Commuting Survey 2016 
Given the rather long and detailed questionnaire, incentives were offered to entice greater participation. Specifically, respondents who completed Part I of the survey were offered the chance to enter a drawing (by entering their email address) for one of ten $\$ 100$ Visa gift cards. To encourage completion of the optional Part II of the survey, participants were told that, by completing the second part, they would receive a second entry into the drawing. Recipients were drawn, winners were contacted, and prizes were mailed in the weeks immediately following completion of the survey.

\subsection{Data processing}

Once the data were finally collected at the end of 2016, data processing and cleaning proceeded during the first few months of 2017. These procedures involved downloading CSV datasets produced by Qualtrics and processing them in R. Nonnumeric data were attributed and converted to the necessary data types (factors). Text entries were analyzed and classified into categories when feasible. Data errors were identified and corrected (when possible) using automated scripts or manually if necessary. Unique anonymous identifiers were added to every record, and records for people who responded to both survey Parts I and II were linked through email addresses or (if missing) nearly coincident survey end and start times.

One of the most challenging aspects of data processing involved the geolocation of respondent-provided home, stop, parking, and work locations. Participants were requested to provide the nearest intersection, neighborhood, or nearby landmark for each location type. However, this information was collected in a single, free-text data entry field, and response formats differed significantly. The geolocation process involved several steps. 
First, all addresses were processed using a long series of text manipulation procedures to generate relatively consistent location text strings. Next, these strings were processed into a format that could be read by the geocoder. Two geocoders were considered: the Google Maps Geocoding API (Google, 2017a) and the Google Places API Text Search (Google, 2017b). Both produced similar results, although more successful matches were found when using the Geocoding API. Custom scripts were written in R (based on Gonzales, 2017) to query Google's API and return necessary geolocation information for each location record: a formatted full address, a coordinate (latitude, longitude), and a unique Google Place ID. Although the automated geocoding procedure produced matches for most locations, about $15 \%$ had errors or were not found. (An interactive web map was developed to check all geocoded locations for errors.) An iterative process fixed locations with errors by manually editing and then feeding them back through the geocoding algorithms until a valid match was found.

An important use for the geocoded home and work locations was the construction of level-of-service information — travel time and cost—-for both the chosen mode and all modal alternatives, for use in the mode choice analysis. This task involved querying the Google Maps Directions API (Google, 2017c) for mode-specific shortest-path travel times between home and work locations, accounting for typical time-of-day traffic conditions. Weather data were also joined to the dataset based on the weather station closest to home using data from the National Centers for Environmental Information (NOAA, 2017). See Chapter 6 for more information on these data augmentation processes. 


\subsection{Results}

Table 3.2 shows the number of responses to both Parts I and II of the Commuting Survey 2016. It also includes the number and percentage of respondents who completed each subsection of the surveys. Note that these response numbers include people who may have skipped some intermediate questions, so the number of nonresponses to a particular question was likely higher.

\section{Table 3.2 Number of respondents completing each section of each survey}

\begin{tabular}{lrr}
\hline Survey section & $\#$ & $\%$ \\
\hline Part I Survey & & \\
Started & 791 & 100 \\
Introduction & 737 & 93 \\
Personal \& transportation characteristics & 723 & 91 \\
Home, job, \& typical commute & 698 & 88 \\
Most recent commute & 679 & 86 \\
Other modes could have used & 657 & 83 \\
Submitted & 651 & 82 \\
Part II Survey & & \\
Started & 521 & 100 \\
Introduction & 513 & 98 \\
Use of different modes & 496 & 95 \\
Multitasking, satisfaction, \& attitudes & 480 & 92 \\
Physical activity levels & 475 & 91 \\
Submitted & 475 & 91 \\
\hline
\end{tabular}

For Part I, 791 people started the survey, but only 651 (82\%) made it to the end of the online questionnaire. Most of this attrition came in the introduction section, where 7\% of people were eliminated because either they were ineligible (i.e., nonworkers, lived outside of Portland) or did not consent, or because they did not move beyond the second page of the survey. (People who clicked the survey link but closed the browser window 
before selecting an answer to the consent question on the first page were not recorded.) The subsequent sections saw only a $2-3 \%$ drop-off rate between each section.

For Part II, 521 people started the survey, or about $80 \%$ of the people who completed the Part I survey. This relatively high follow-up rate suggests that the additional incentive (another entry into the drawing) was attractive and/or people were interested in this topic and wished to share more information about their commuting experiences. Of the people that started the Part II survey, 475 (91\%) went through all sections to the end. Again, each section saw about $2-3 \%$ of respondents drop out.

Figure 3.3 summarizes the times respondents took to complete both Parts I and II of the survey. The box plots show a thick horizontal line at the median, the interquartile range (25th to 75 th percentiles) within the box, and whiskers extending to 1.5 times the interquartile range; outliers are represented by dots located beyond the whiskers. (Some outliers are not shown as they lie beyond the range of the plot.) These times are for only people who completed and submitted each survey. They are also technically the difference between the time when the survey was started and the time when the survey was submitted. Therefore, they may exaggerate the actual amount of time people spent taking the survey, and average survey completion times would likely be less if one were to account for pauses, interruptions, and people who quit and later returned to finish the survey. The median respondent completed the Part I survey in 29 minutes; the middle 50\% completed it in between 22 and 41 minutes. The estimation completion time of 30 to 40 minutes provided at the start falls well within this empirical range of response times. For the Part II survey, the median respondent finished in 18 minutes; the middle 50\% completed it in 14-24 minutes. Again, the estimated time for this optional part was 15-20 minutes, perfectly 
within the observed range. Overall, participants were given realistic estimated completion times at the beginning of each survey.

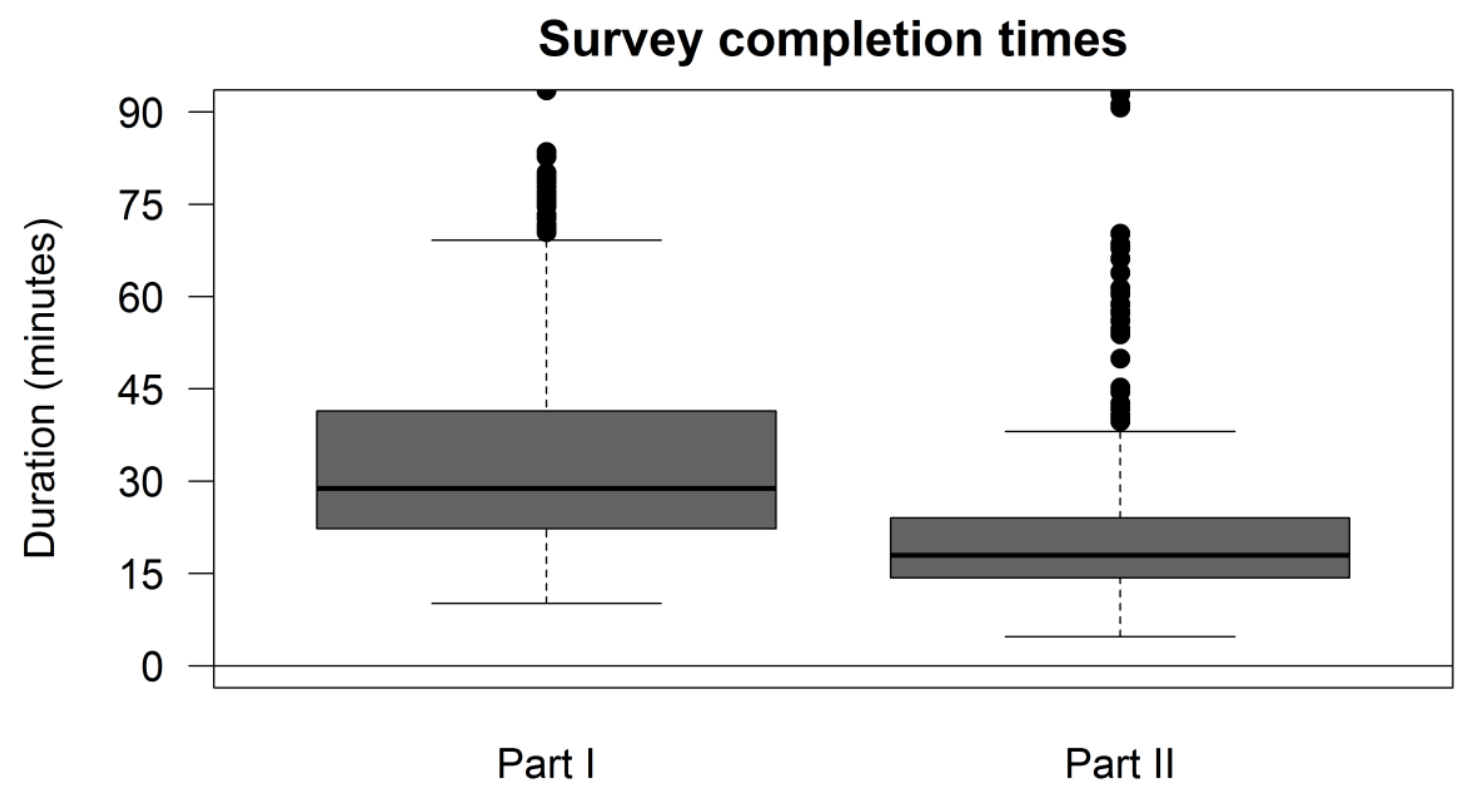

Figure 3.3 Box-and-whisker plots of survey completion times

The commuting survey was live and accepting responses from 18 October 2016 to 3 January 2017. Most responses were received before 16 December 2016, the deadline for respondents to be entered into the drawing for the gift card incentives. The survey remained open for approximately two more weeks for any straggling or incomplete responses, although very few were received. Figure 3.4 displays the number of respondents starting the Part I survey by date. Peaks were found on Mondays of nearly every week. The greatest number of responses (240) were received on Tuesday 1 November 2016, the same day that the City of Portland SmartTrips email list was contacted. The final week also saw higherthan-normal responses, corresponding with the field recruitment efforts. 


\section{Frequency of responses by date}

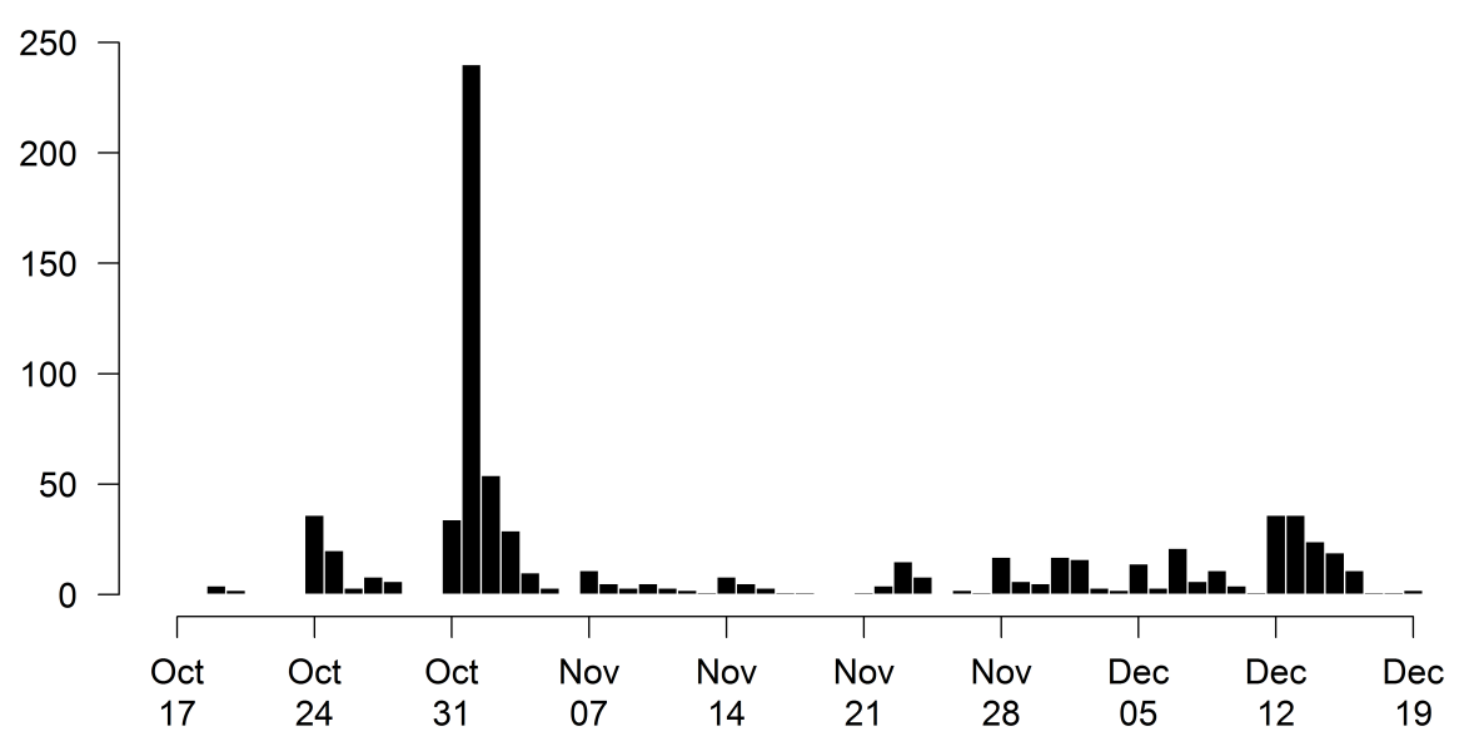

Figure 3.4 Frequency of survey responses by date

True response rates were unable to be computed due to the nature of the participant recruitment methods. The survey invitation emails reached an unknown number of employees. However, rough response rates to the SmartTrips email and the field recruitment can be calculated by making some assumptions. Presuming $80 \%$ of the responses on 1 November and the subsequent days of the same week were from the SmartTrips email list, the response rate would be approximately: $80 \% \times 333$ responses $=$ 266 responses $\div 3,700$ emails $=7.2 \%$. Similarly, assuming $60 \%$ of the responses on 13 December and subsequent days of the same week were from postcards handed out during the field recruitment, an approximate response rate would be: $60 \% \times 90$ responses $=54$ responses $\div 166$ postcards $=32.5 \%$. These approximate response rates $-5-10 \%$ for a direct email, $25-40 \%$ for postcard handout — appear reasonable and expected. 


\subsubsection{Representativeness}

To assess the representativeness of the sample, descriptive statistics for key variables in the sample were compared to similar population-level values. These comparative descriptive statistics-shown in Table 3.3-were calculated for the population using data from the 5-year 2011-2015 American Community Survey (ACS) (US Census Bureau, 2017) for the Portland, OR-WA urbanized area. All categorical variables in the sample had significantly different distributions than the same variables in the population — based on chi-square tests of independence — but some differences were more substantial than others.

With respect to trip characteristics, the sample was not necessarily representative of the greater Portland-area commuting population, although this was by design. Notably, the sample contained a smaller proportion of auto commuters than the region at large; instead, people commuting by bicycle and public transit made up a greater share of the sample than their population shares would have indicated. Indeed, the survey was not a random sample: It was designed to capture a larger share of nonauto commuters so that the mode choice model could be estimated and sensitivities between different modes could be examined. As described above, efforts were made to recruit participants from Downtown Portland and other areas where nonauto transportation mode options may have been more attractive and available. Overall, the commutes measured by the survey took about 10 minutes longer than average Portland-area commutes; this difference could be partially explained by the much larger proportion of transit trips, which tend to be longer in duration. 


\section{Table 3.3 Comparative descriptive statistics}

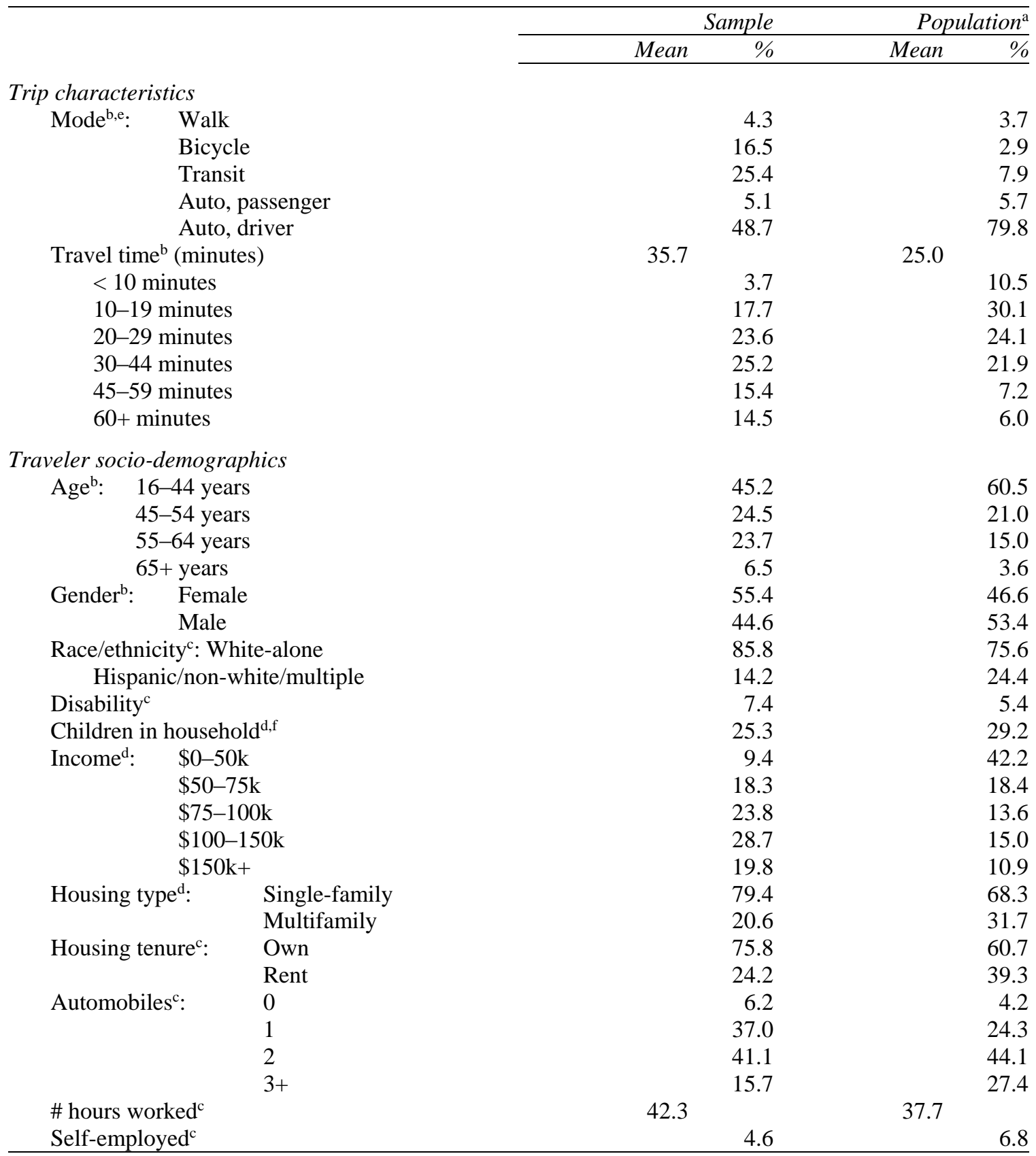

${ }^{a}$ From the 5-year 2011-2015 American Community Survey for the Portland, OR-WA urbanized area.

${ }^{\mathrm{b}}$ For population estimates, these values were among workers who did not work at home.

${ }^{\mathrm{c}}$ For population estimates, these values were among all workers.

${ }^{\mathrm{d}}$ For population estimates, these values were among all (working and non-working) households.

e For population estimates, "Auto, driver" included everyone who drove alone, half of those who carpooled, and half of those in the category "Taxicab, motorcycle, or other means." "Auto, passenger" included half of those who carpooled and half of those in the category "Taxicab, motorcycle, or other means."

${ }^{\mathrm{f}}$ For sample values, these included children aged 16 or less. For population estimates, these included children aged 17 or less. 
Considering socio-demographic characteristics, the commuters captured by the survey more closely matched expected proportions from a representative and random sample of Portland-area commuters. The sample contained a slightly greater-than-expected number of women, older workers, and people of white non-Hispanic race/ethnicity. It also was slightly under-representative of renters and people living in multifamily housing. Survey respondents worked slightly more hours, on average, and owned fewer cars than Portland-area workers. The sample roughly matched the expected number of people with a disability, people with children, and the self-employed.

The biggest sample deviation from the population was on household income: The sample contained nearly twice as many people with incomes greater than $\$ 75,000$ and only a quarter of people with the lowest incomes $(<\$ 50,000)$ than would have been expected from a random selection of households. The true deviation was actually less than this: The corresponding ACS values were for all Portland-area households, so it would be expected that some of the lowest-income bracket contains nonworking households. Nevertheless, some of this higher-income bias likely remains in the sample. (It also may have been manifested in the higher proportions of single-family and owner-occupied housing.) This higher-income skew is likely the result of sampling and response biases: The survey reached many downtown office workers, government employees, and people working in suburban high-tech manufacturing (see information on work locations in the following paragraphs), occupations and industries that may pay higher average wages. Additionally, higher-income workers may have been more inclined to complete a lengthy survey than lower-income workers, who may have been less likely to have the free time or access to a computer to take the survey. The difficulty of reaching a lower-income population is a 
common problem with travel surveys (Bradley, Bergman, Lee, Greene, \& Childress, 2015), and something that future studies of the PUT concept should try to overcome.

Besides the modal differences that yielded greater responses by nonauto commuters (by design) and the inclusion of a disproportionate share of higher-income commuters (as a result of sampling and response biases), overall, the Commuting Survey 2016 captured a relatively-representative sample of commuters in the Portland, OR, region.

The geocoded home and work locations of survey respondents are mapped in Figure 3.5. Places of residence were scattered throughout the region. Although many respondents lived in inner North, Northeast, and Southeast Portland (between the Willamette River and Interstate 205), this area is among the denser parts of the region and contains a large population. There were also concentrations of respondents from Downtown Portland. Responses were not exclusive to the City of Portland; in fact, many respondents lived in various places throughout Washington County, to the west. Relatively fewer responses were received from people living in suburban areas of east Multnomah County, Clackamas County (to the southeast), and Clark County, Washington (north of the Columbia River). Work locations were more spatially concentrated than home locations, as are jobs in the Portland region. A large number of respondents worked in the Central City of Portland, particularly Downtown and the Lloyd District. This is not surprising, considering the high concentration of jobs in this part of the region and the large number of respondents that were recruited through the SmartTrips downtown email blast and the field recruitment efforts. Other concentrations of respondents were found in other job centers throughout the region, including Swan Island, Portland International Airport, the high-tech campuses of Washington County, Downtown Hillsboro, and Tigard. 

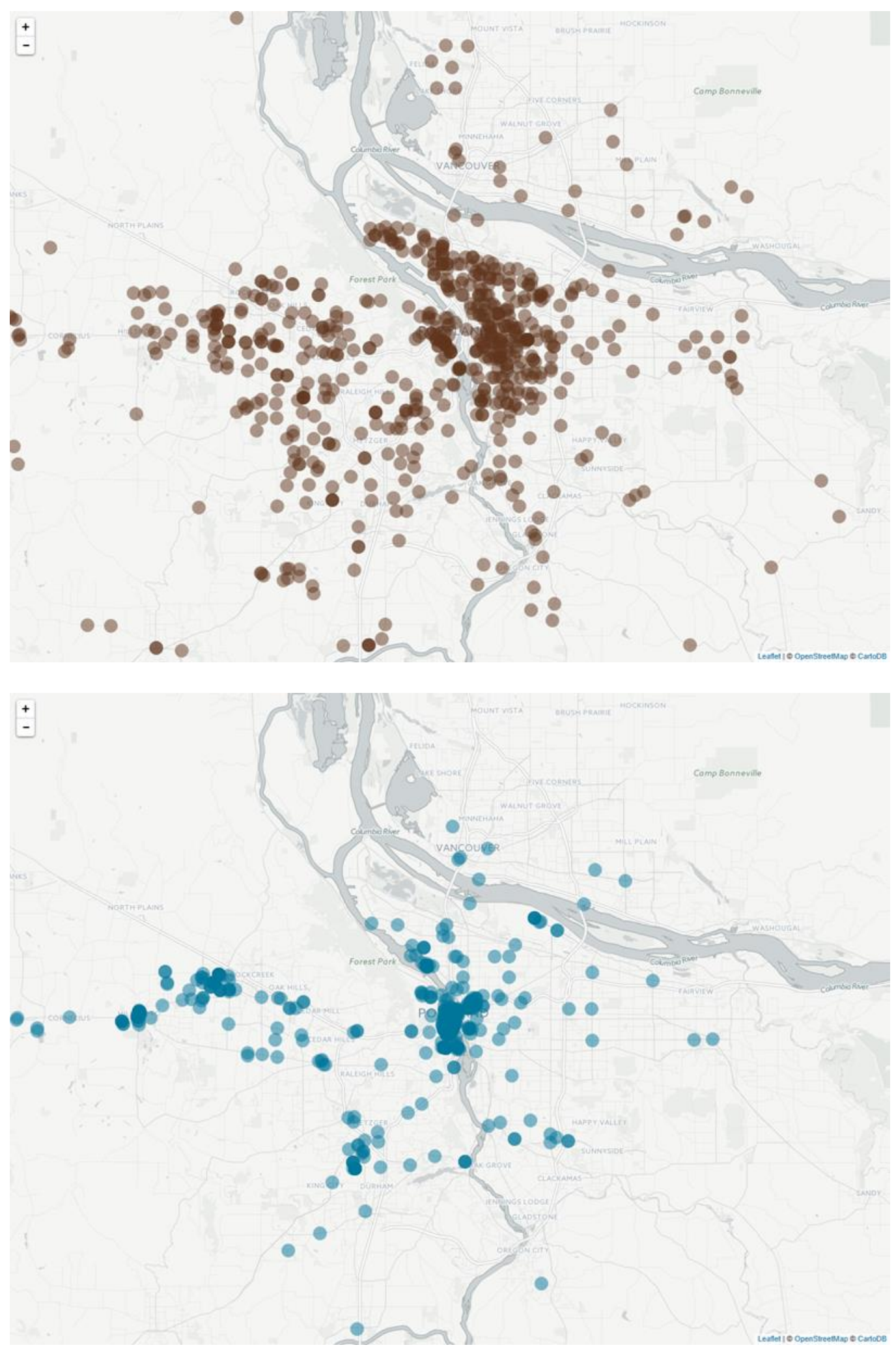

Figure 3.5 Home (upper) and work (lower) locations for survey respondents 
Overall, the responses to the Commuting Survey 2016 exhibited a reasonable amount of variety in terms of locations and commutes. The survey captured a large number of people commuting to Downtown, which was useful for the mode choice analysis because nonautomobile commutes are much more common there. It also gathered responses from many suburban residents and suburban workers. Overall, the recruitment methods yielded a sample that was relatively reflective of the variety of commuting experiences that can be found in the Portland, Oregon, metropolitan area.

\subsubsection{Travel-based multitasking}

As mentioned in the Introduction and Questionnaire sections, the travel activity aspects of the PUT concept—also known as travel-based multitasking—were measured in several ways. The activity participation question measured what sorts of activities people reported doing while commuting. For each of these activities, people then reported about activity duration, or the percentage of travel time spent doing each activity. Finally, an overall assessment of travel usefulness was gathered. The figures below summarize key survey results about travel-based multitasking. More detailed results can be found in Chapter 4 .

Figure 3.6 depicts the top 10 most frequently reported activities among all commuters in this study. Over half (53\%) of travelers said they listened to some sort of audio, including music or via the radio. About $45 \%$ of people reported doing at least one of two kinds of passive activities: thinking or daydreaming and viewing scenery or watching people. All other activities were reported by fewer than a quarter of respondents. 


\section{Activities}

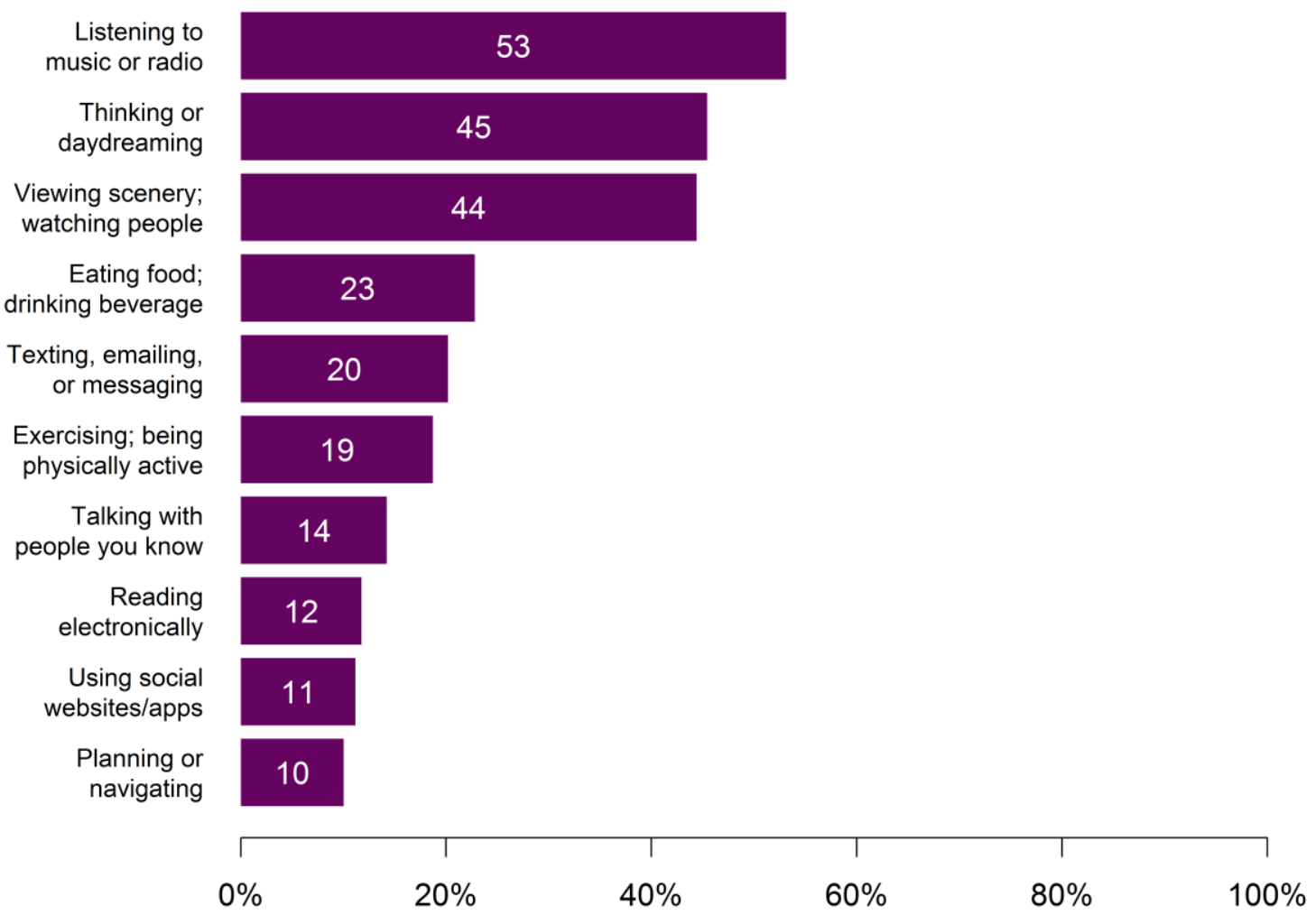

\section{Figure 3.6 Frequently reported activities overall}

Figure 3.7 displays responses to the travel usefulness question, summarized by commute mode. Overwhelmingly, most people walking (87\%) and bicycling (94\%) reported having at least somewhat useful commutes, with the most useful commutes experienced by people bicycling (68\% mostly useful). Slightly more than half of transit commuters (57\%) and auto passengers (54\%) reported useful commutes, while about half of auto drivers felt like their commuters were at least somewhat wasted time (50\%). There appear to have been significant modal differences in reported travel usefulness. 


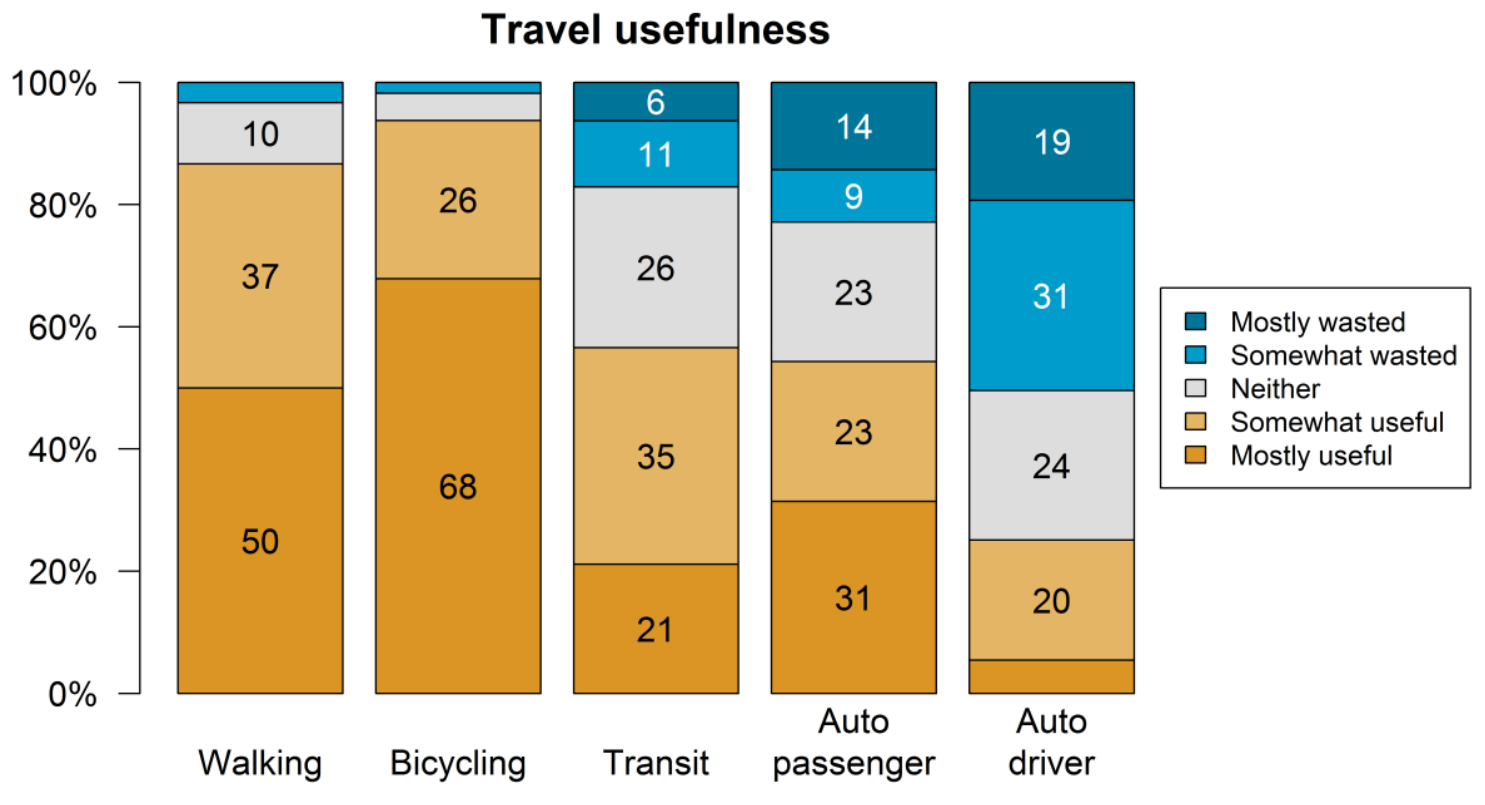

Figure 3.7 Travel usefulness by commute mode

\subsubsection{Travel subjective well-being}

The travel experience aspects of the PUT concept—also known as subjective wellbeing (SWB) in the travel domain-were also measured in several different ways. Questions about travel affect measured feelings, moods, and emotions that may have resulted from the commute. Questions about travel eudaimonia inquired about the extent to which commuting helped fulfill some desire, express a trait, or improve a skill. The Satisfaction with Travel Scale (STS) included several questions to identify overall levels of commute satisfaction and hedonic SWB. Finally, an overall assessment of travel liking was gathered. The figures below summarize key results about travel subjective well-being. More detailed results can be found in Chapter 5.

Figure 3.8 depicts the 10 travel affect items that were most frequently reported to have been felt "at least a little" among all commuters in this study; green adjectives are 
positive, and red ones are negative. Most travelers (over 80\%) said they felt both Alert and Attentive while commuting. About $60 \%$ of people reported feeling Calm for at least part of their journey. Items related to positive affect that were reported somewhat frequently included feeling Active (47\%) and Determined (35\%). Some negative emotions were also somewhat frequently reported: feeling Stressed (40\%) and Frustrated (35\%). All other feelings were reported by fewer than a third of respondents. These results suggest that positive emotions outweighed negative emotions, at least in terms of frequency.

\section{Travel affect}

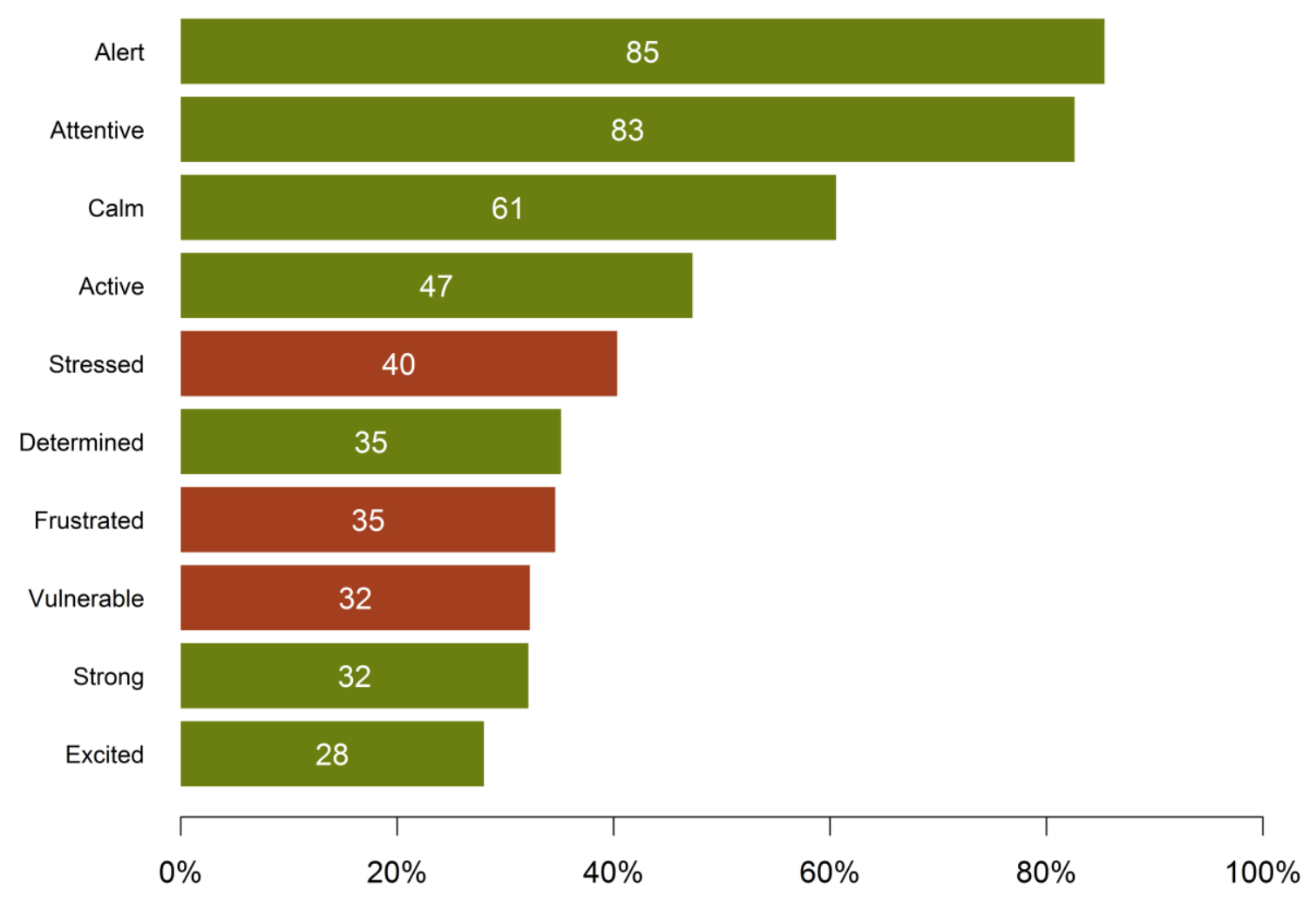

Figure 3.8 Frequently reported travel affect items overall 
Figure 3.9 shows the 10 most frequently reported travel eudaimonia items among all commuters in this study. (All of these items are assumed to have contributed positively towards SWB, so color distinctions were not used.) No items stand out strongly. Feelings about the commute being A routine or A buffer between home and work were most common and were reported by about 55\% of travelers. About 50\% of people reported that their journeys helped express or fulfill their desire for Freedom and Independence, and about $45 \%$ felt in control. Other items that were somewhat frequently reported included Comfort (40\%), Privacy (33\%), Mental health (36\%), and Environmental values (35\%). All other items were selected by fewer than a third of respondents. 


\section{Travel eudaimonia}

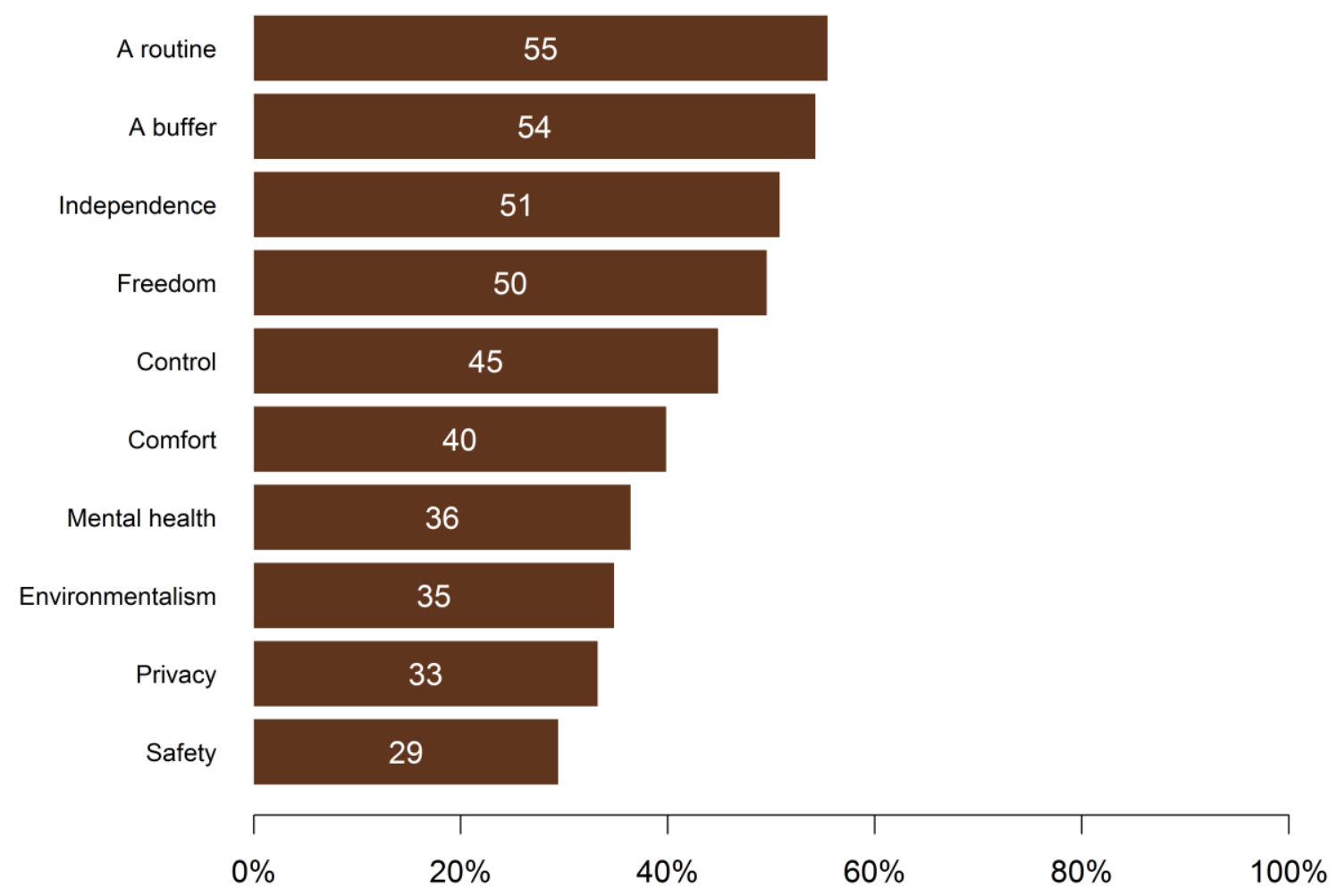

\section{Figure 3.9 Frequently reported travel eudaimonia items overall}

Figure 3.10 presents the distributions of averaged standard scores for the STS, summarized using box-and-whisker plots for each commute mode. After averaging each respondent's scores on the STS's nine items, the scores were standardized: i.e., centered on the mean and scaled by the standard deviation. The box plots for each group show a thick horizontal line at the median, the interquartile range (25th to 75 th percentiles) within the box, and whiskers extending to 1.5 times the interquartile range; outliers are represented by dots located beyond the whiskers. There appear to have been significant modal differences in commute satisfaction. Overall, bicycling and especially walking commuters 
reported higher than average travel satisfaction ratings. Transit riders and auto passengers had roughly average ratings, and auto drivers had the lowest STS scores, on average. Despite these modal trends, there was large variation in STS scores even within modes.

\section{Satisfaction with Travel Scale}

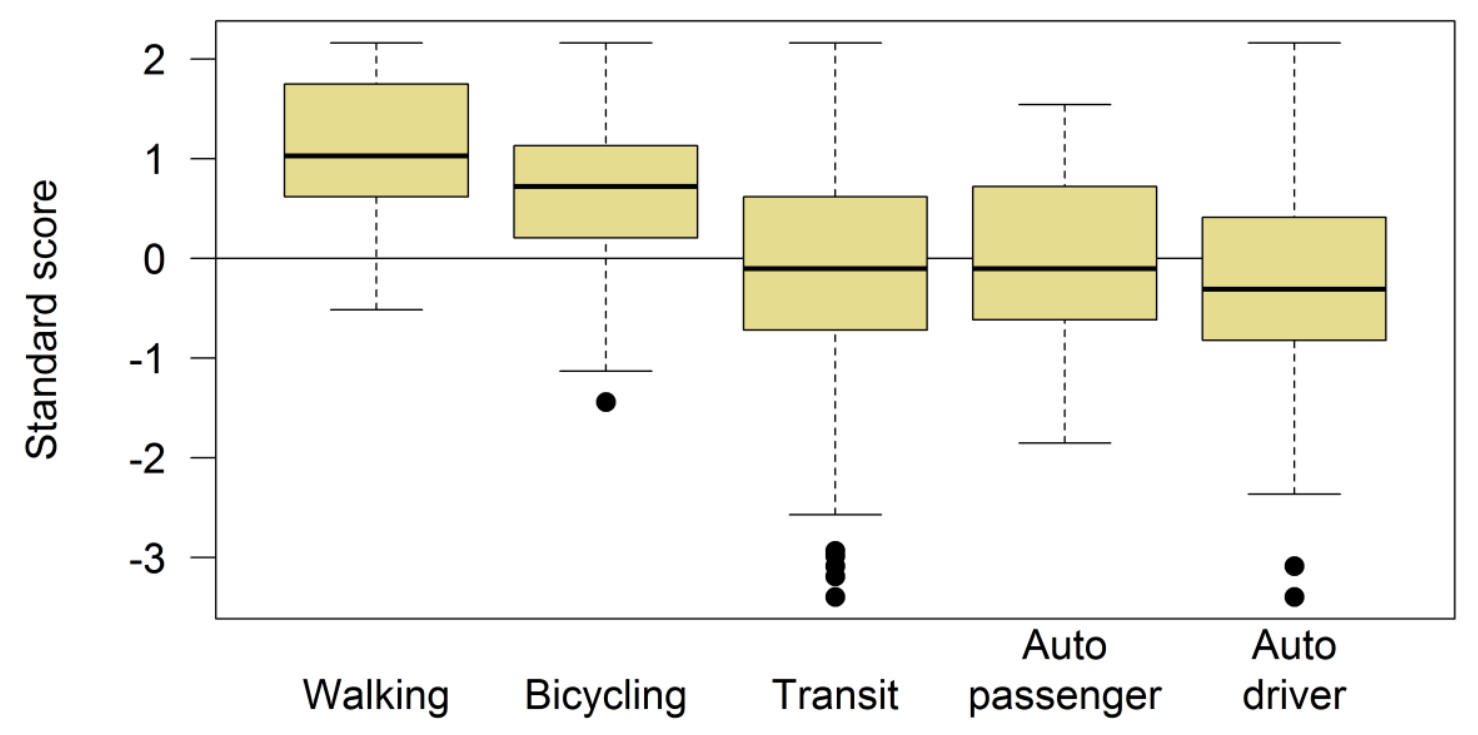

Figure 3.10 Box-and-whisker plot of averaged STS scores by commute mode

Figure 3.11 displays responses to the travel liking question, summarized by commute mode. Overwhelmingly, most people walking (93\%) and bicycling (95\%) reported liking their commutes, with most of these people strongly liking their commutes. About two thirds (66\%) of auto passengers liked their commutes. Slightly more than half of transit commuters (57\%) and slightly less than half of auto drivers (45\%) liked their commutes. While auto drivers were the least likely to like commuting, only $21 \%$ reported somewhat or strongly disliking the commute. There appear to have been significant mode differences in reported travel liking. 
Travel liking

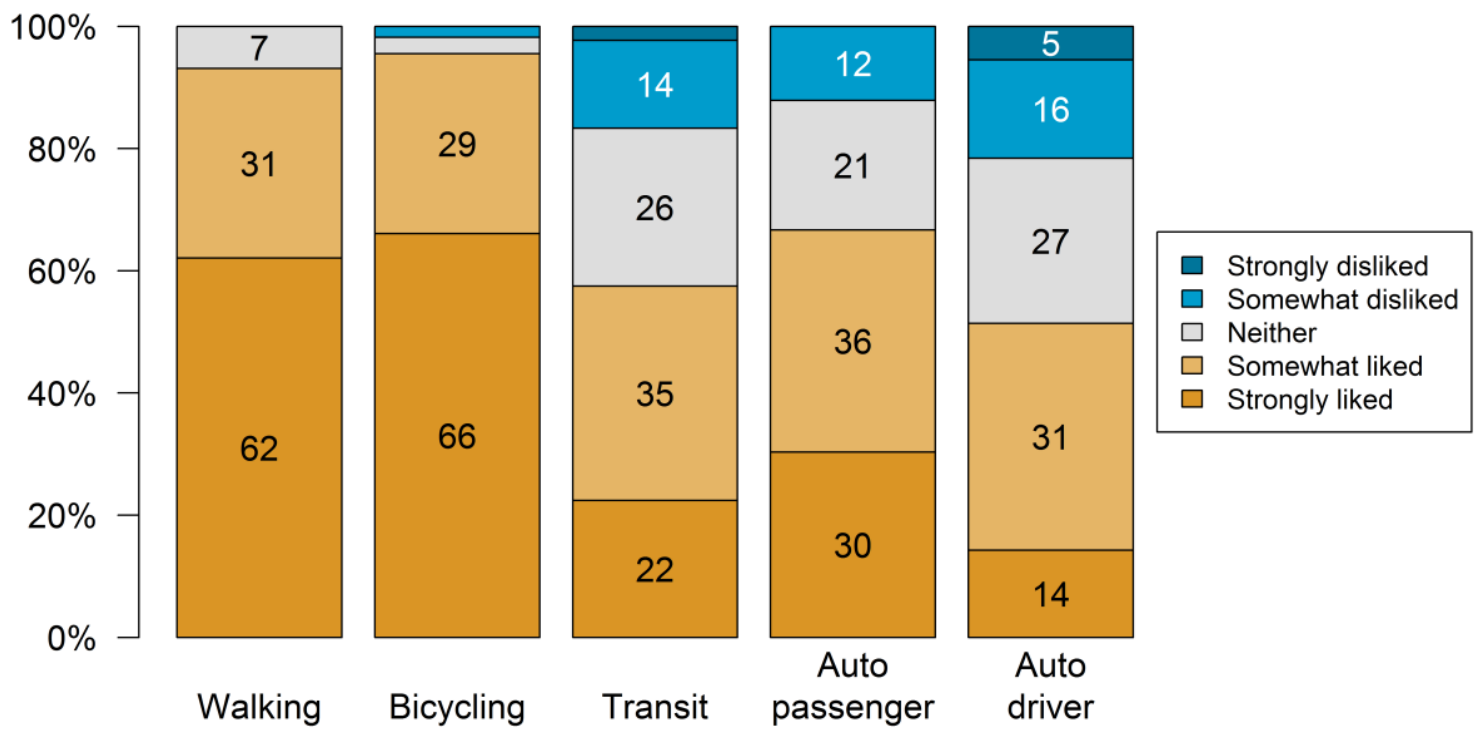

Figure 3.11 Travel liking by commute mode

\subsubsection{Summary}

In summary, the Commuting Survey 2016 successfully gathered information about the positive utility of travel (PUT) concept—including both travel activity and travel experience aspects - from approximately 650 commuters in the Portland, OR, metropolitan area. The results of the survey are further analyzed in Chapter 4, Chapter 5, and Chapter 6; see those chapters for more detailed results. 


\section{Chapter 4 Travel activities}

\section{Making use of the commute: Travel-based multitasking in Portland, Oregon}

\subsection{Abstract}

The "positive utility of travel" concept suggests that one way people benefit from travel is by engaging in activities while traveling. This study investigates the twin topics of travel-based multitasking and travel usefulness, using the results of a survey of about 650 commuters in the Portland, Oregon, region. In estimating binary logit models of participation in several different types of activities (grouped using exploratory factor analysis) and ordered logit models of subjective assessments of travel usefulness, this analysis examines differences by commute mode and various traveler characteristics. Walking and bicycling commuters found their commutes to be the most useful, apparently because they valued exercising. Auto drivers had the most wasteful commutes; most only listened to audio. Transit riders and auto passengers engaged in a greater number and variety of activities while traveling - including ICT-based activities - than users of other modes. Although age was negatively associated with ICT activities, listening to music, and travel usefulness, few other sociodemographic attributes were consistently significant. Instead, traveler perceptions appeared to play a bigger role. Study findings suggest that some people do make use of their commute travel time through travel-based multitasking, while others travelers may instead be doing things just to kill time. This research offers implications for understanding the behavior and time use of transit passengers and people walking and bicycling, and for anticipating future technological developments. 


\subsection{Introduction}

Traditional transportation analysis methods, including those that underlie travel demand forecasting models and tools for transportation project appraisal, assume that the demand for passenger transportation is derived from the demand for conducting activities in spatially distinct locations. A corollary of this axiom is that travel time is a disutility that travelers desire to minimize. As a result, the primary user benefit of large, mobilityenhancing transportation projects is the aggregate value of travelers' marginal travel time savings. Over the past two decades, scholars have questioned the universality of these assumptions, instead suggesting and providing evidence that some travel may be motivated by factors other than reaching activity destinations and that some people may benefit from the act of traveling itself. These perspectives are known as the positive utility of travel concept (Salomon \& Mokhtarian, 1998; Mokhtarian \& Salomon, 2001).

One major component of the positive utility of travel is travel-based multitasking: doing other activities while traveling (see Chapter 2). (A second aspect includes positive travel experiences, expressed by positive emotions or symbolic fulfillment from traveling.) People who do things while traveling presumably find some benefit in these activities. They may be making productive use of their travel time (Lyons \& Urry, 2005) by doing traditional work, maintenance, or leisure activities: writing or reviewing documents, eating a meal, reading a novel, etc. For some, traveling (and commuting in particular) can be a time of transition (Jain \& Lyons, 2008), providing a buffer between home and work and allowing the traveler to mentally prepare for obligations at the destination, or a time to relax and escape from such obligations. People who use their commutes to snooze or sleep, think, daydream, or stare out the window at the passing (natural or urban) landscape may 
have some of these goals in mind. Still other activities may be less about productivity or mental health and more about making travel less onerous or more enjoyable: Checking social media, playing a game, and listening to music are all activities that can reduce the disutility of traveling.

One question arising from the study of travel-based multitasking is about travel usefulness: How much do people value the activities they conduct while traveling? This question is especially relevant considering the importance of the value of travel time savings (VTTS) for transportation project appraisal (Mackie et al., 2001). This measure of the willingness to pay for a marginal reduction in travel time is usually derived from travelers' revealed or stated preferences when faced with tradeoffs between travel time and cost, and it is an important input to the cost-benefit analysis of major mobility-enhancing infrastructure investments. If travelers value multitasked activities, then current VTTS estimates may be biased, yielding incorrect predictions of travel behavior shifts and calculations of user benefits. Work is underway to more formally consider activity participation during travel within microeconomic time use and allocation theories (Pawlak et al., 2015), and there is emerging research that suggests travel-based multitasking may indeed affect VTTS (Ettema \& Verschuren, 2007, Singleton \& Clifton, 2015). Understanding the usefulness of travel activities is an important part of these efforts.

Research on travel-based multitasking has increased in recent years (Kenyon \& Lyons, 2007). One reason for the rising interest in multitasking during travel is the coincident development of more advanced information and communications technologies (ICTs) that have increased the availability and use of internet-enabled electronic devices. Items like smartphones and tablet computers have shifted the media by which some 
activities_-reading, playing games, etc.-are often done while simultaneously increasing the possible range of mobile activities. ICT devices have put the expanding universe of telework, teleshopping, social networking, gaming, and other activities in travelers' palms.

Travel-based multitasking is particularly relevant for understanding the time use patterns and motivations of bus, rail, and other transit riders. Compared to most other travelers (besides auto passengers), people riding transit have the most flexibility for doing other things while on the go because they do not have to dedicate as many physical or mental resources to the transportation (driving, operating, or walking) task. It is therefore not surprising that most studies have investigated transit passengers, finding that they engage in more frequent and a greater variety of activities, including productive, relaxing, and ICT-based activities. People riding transit may have the best opportunity to make use of their travel time.

People walking and bicycling also gain while traveling: by exercising and being physically active. The ability to be physically active while on an otherwise mandatory trip (i.e., to work) is a good example of travel-based multitasking and the usefulness of travel. Some people may even substitute an active commute for a gym membership or participation in organized sports. The physical activity benefits of walking and bicycling may be an important key to better understanding active travel behavior.

\subsubsection{Research questions}

This study investigates a number of research questions related to travel-based multitasking: How does activity participation during travel vary across modes: auto drivers and passengers, transit riders, and people walking and bicycling? What groups of activities 
are commonly done together? What trip and traveler characteristics are associated with travel-based multitasking? A second group of research questions is concerned with travel usefulness: How useful is travel-based multitasking? How much do people value the time they spend engaged in activities while traveling? Which activities are considered most useful? What trip and traveler characteristics are associated with travel usefulness?

This study answers these questions by analyzing the results of a 2016 survey of commuters in the Portland, Oregon, region. The chapter is structured as follows. First, literature on travel-based multitasking, travel usefulness, and their associated factors is reviewed. Next, the data and methods are summarized. Results of several binary logit models of activity participation as well as ordinal logit models of travel usefulness are then presented. Finally, the results and implications of this study are discussed, including opportunities for future work.

\subsection{Literature review}

\subsubsection{Travel-based multitasking}

Activity participation during travel — travel-based multitasking-is a subset of multitasking, which is located within a broad body of research on time use and human performance. Understanding multitasking behavior can be challenging, and studies have faced several limitations: defining multitasking in different ways; confounding multitasking (the behavior) with polychronicity (the preference for multitasking); mixing measures of activity participation with measures of item use; confusing shares of resources and shares of time dedicated to different activities; measuring a limited number of 
activities; trying to distinguish primary from secondary activities; and trying to classify distinct activities that may be closely related (Circella et al., 2012; Kenyon, 2010; König $\&$ Waller, 2010). There were few studies of multitasking during travel until the first decade of the 21st century (Kenyon \& Lyons, 2007).

Since then, a growing number of studies have measured or focused on travel-based multitasking. Two data collection methods predominate. The first method uses passive field observations, in which an observer travels with participants and records what travelers appear to be doing and for how long. Observations often take place surreptitiously on board public transit vehicles (Guo et al., 2015; Ohmori \& Harata, 2008; Russell et al., 2011; Timmermans \& van der Waerden, 2008; van der Waerden et al., 2009). The second method asks participants to fill out questionnaires or activity diaries and recall what they were doing on a recent trip. While most studies utilizing this latter method still focus on public transit passengers (Ettema et al., 2012; Frei et al., 2015; Guo et al., 2015; Lyons et al., 2007; Lyons et al., 2013; Lyons et al., 2016; Ohmori \& Harata, 2008; Yosritzal, 2014; Zhang \& Timmermans, 2010), an increasing number are starting to analyze travel-based multitasking across all transportation modes (Berliner et al., 2015; Circella et al., 2015; Ettema \& Verschuren 2007; Keseru et al., 2015; Malokin et al., 2015). A handful of studies use interviews or focus groups to measure travel-based multitasking (Handy et al., 2005; Jain \& Lyons, 2008).

If an activity can be done in life with only minimal external resources and objects, it can likely also be done while traveling: talking, reading, writing, listening to audio, eating, drinking, viewing scenery, sleeping, etc. New information and communications technologies (ICTs) - including internet-enabled devices like smartphones-have 
increased the range of potential multitasked travel activities and changed the items people use to conduct certain activities (e.g., reading, playing games). These points notwithstanding, several scholars have taken on the challenge of enumerating and categorizing activities (Circella et al., 2012; Circella et al., 2015; Kenyon, 2006; Kenyon \& Lyons, 2007; Keseru et al., 2015; Lyons et al., 2013; Malokin et al., 2015; Ohmori \& Harata, 2008; Timmermans \& van der Waerden, 2008). Some activities are grouped by purpose —work/study, maintenance, and leisure—or function: communicating, reading, writing, using media, doing nothing, etc. Another way to classify activities is by the degree to which they require "the deliberate use of one's physical and/or mental faculties" (Circella et al., 2012, p. 83). Activities requiring little or no input from the individual are more passive; activities demanding significant investment of physical or mental resources are more active.

Some trip and travel characteristics have frequently been associated with travelbased multitasking. In multimodal studies (Berliner et al., 2015; Circella et al., 2015; Ettema \& Verschuren 2007; Keseru et al., 2015; Malokin et al., 2015), activity participation varies across modes, with some of the biggest differences found between modes requiring more active attention or operation and those that require more passive attention (Circella et al., 2015). Car drivers are more likely to be listening to music or other audio; on the other hand, reading, writing, resting, and sleeping are more prevalent among train, bus, or car passengers. Some more passive activities (viewing scenery, watching people, thinking, and daydreaming) are common to all modes, while exercising is almost exclusively reported by people walking and bicycling. 
In general, travel-based multitasking appears to increase with travel time (Berliner et al., 2015; Lyons et al., 2007; Ohmori \& Harata, 2008; Zhang \& Timmermans, 2010), perhaps because longer activity durations can be of higher quality, or because travelers with longer trips have a bigger incentive to make productive use of their time. Trip purpose may also play a role (Frei et al., 2015; Keseru et al., 2015; Lyons et al., 2007; Lyons et al., 2013; Lyons et al., 2016), as traveling can help people prepare for or relax before performing an important activity at the destination. People traveling alone might be slightly less likely to participate in activities during the trip (Timmermans \& van der Waerden, 2008; van der Waerden et al., 2009; Zhang \& Timmermans, 2010).

Studies examining demographic and socioeconomic traveler characteristics as determinants of travel-based multitasking reveal only a few consistent findings. Younger travelers appear more likely to do activities involving smartphones and other electronic devices, while older travelers are more likely to read (paper) books or newspapers (Berliner et al., 2015; Frei et al., 2015; Guo et al., 2015; Lyons et al., 2016; Russell et al., 2011). Some evidence suggests that women are more likely to spend some time talking or communicating, while men are more likely to perform work-related activities (Berliner et al., 2015; Keseru et al., 2015; Lyons et al., 2007; Lyons et al., 2013; Lyons et al., 2016; Russell et al., 2011). The few travel-based multitasking studies that did include traveler attitudes and personalities found some significant associations, such as between technology-oriented travelers and ICT-enabled activities (Berliner et al., 2015). 


\subsubsection{Travel usefulness}

Some travel-based multitasking questionnaires also ask travelers to assess the value, worth, or usefulness of a trip (Circella et al., 2015; Lyons et al., 2007; Lyons et al., 2013; Lyons et al., 2016; Rosenfield \& Zhao, 2016; Susilo, Lyons, Jain, \& Atkins, 2012). In general, only $10-30 \%$ of travelers view traveling as mostly wasted time, while roughly $20-30 \%$ report travel time to be very worthwhile or mostly useful. Younger travelers appear more likely to consider traveling to be wasted time. People doing traditional workrelated activities (reading, writing, or emailing) are more likely to see travel time as being useful, and people doing more passive activities (window-gazing, people-watching) are more likely to report wasted time, although these results could vary by mode (Circella et al., 2015).

It is reasonable to assume that questions of travel usefulness are attempting to measure an overall assessment of the value of travel-based multitasking. However, when considering the usefulness of a trip, respondents may confound the intended benefits of multitasking (e.g., productive use of travel time, preparation for a destination activity) with enjoyment of the travel experience or the instrumental benefits of reaching a destination (see Chapter 2). This possibility should considered when analyzing travel usefulness.

\subsection{Data and methods}

The analyses presented in this chapter are part of a broader study investigating the positive utility of travel (PUT) concept and the effects of a PUT on mode choice. This PUT study included a 30-minute online questionnaire survey administered to working and commuting adults in the Portland, Oregon, region. Respondents were asked to report 
detailed information about their most recent commute trip from home to work, including responses to questions on travel-based multitasking and travel usefulness. Data were collected between mid-October and mid-December 2016, and participants were primarily recruited via email at their place of employment. Although 791 people started the survey, only 656 people completed enough questions to be used in these analyses. For more information on the data collection process, see Chapter 3. Descriptive statistics of the sample are shown in Table 4.2.

The questionnaire approach was selected to measure travel-based multitasking. This method avoids a number of measurement challenges associated with passive observational studies — see Guo et al. (2015) for a recent summary — and allows for a deeper probing of multiple activities that can be done using a common item (e.g., smartphone), although it could suffer from recall or response biases. Survey questions focused on activity engagement (e.g., listening to music) rather than item use (e.g., using a smartphone), because the former may more clearly illuminate motivations for multitasking than the latter (see Chapter 2), and because this was not an observational study where item use is more easily measured. Supplemental questions about item use or possession (Lyons et al., 2016) were unable to be included due to restrictions on the survey length. More detailed questions about the quality of travel-based multitasking (Rosenfield \& Zhao, 2016) were considered but excluded for the same reason.

Respondents were first asked to select from a list of activities the things they did while traveling on their most recent commute to work, including everything they did after leaving home until arriving at work. The instructions explicitly stated to include things done "while on board, getting to/from, and waiting for public transit," so this analysis is 
unable to distinguish between activities done while using the primary mode versus those done on access modes or while waiting. Next, respondents reported the approximate percentage of their commute travel time $(0-100 \%$ in $10 \%$ increments) they spent doing each of the selected activities.

Activity options presented to respondents were selected after compiling a master list of activities used in several prior travel-based multitasking questionnaires (Circella et al., 2015; Ettema et al., 2012; Ettema \& Verschuren, 2007; Guo et al., 2015; Kenyon, 2006; Keseru et al., 2015; Lyons et al., 2007; Lyons et al., 2013; Malokin et al., 2015; Ohmori \& Harata, 2008; Russell et al., 2011; Timmermans \& van der Waerden, 2008; van der Waerden et al., 2009; Yosritzal, 2014; Zhang \& Timmermans, 2010). From this list, 23 activities were selected based on their prevalence in the literature, frequency of reported participation in past studies, and breadth in covering a range of different kinds of activities (see Chapter 3). See Table 4.1 for the list of activities. Respondents were also offered up to three "other" options for which to give a text response.

Because of the large number (23) of activities to analyze, activities were grouped using exploratory factor analysis (EFA). One recent study on travel-based multitasking (Malokin et al., 2015) also used EFA to group similar activities; most other studies categorize activities using author judgement (Kenyon \& Lyons, 2007; Keseru et al., 2015; Timmermans \& van der Waerden, 2008; van der Waerden et al., 2009). The EFA used Horn's parallel analysis to determine the approximate number of factors, ordinary least squares minimum residual to extract factors, and oblique oblimin rotation. (Principal axis factoring was considered, but a Heywood case was detected.) The EFA was conducted using the paran (Dinno, 2012) and psych (Revelle, 2017) packages in R. 
Table 4.1 presents the results of the exploratory factor analysis. Prior to conducting the EFA, five activities were removed due to low frequencies of response $(<15)$. Six factors were extracted that together explained about $34 \%$ of the observed variance. However, only two of the factors had multiple items with moderate loadings ( $\geq 0.40)$. The "ICT" activity factor included texting/emailing/messaging, reading electronically, and using social websites/apps, which are all activities facilitated by internet-connected devices like smartphones. The "passive" activity factor included viewing scenery or people watching and thinking/daydreaming. Interestingly, these factors are similar to the "technological" and "recreational" factors identified by Malokin et al. (2015). Instead of calculating factor scores, two new activities were constructed based on participation in one-or-more of the activities that loaded on each factor. 


\section{Table 4.1 Results of exploratory factor analysis of activity participation}

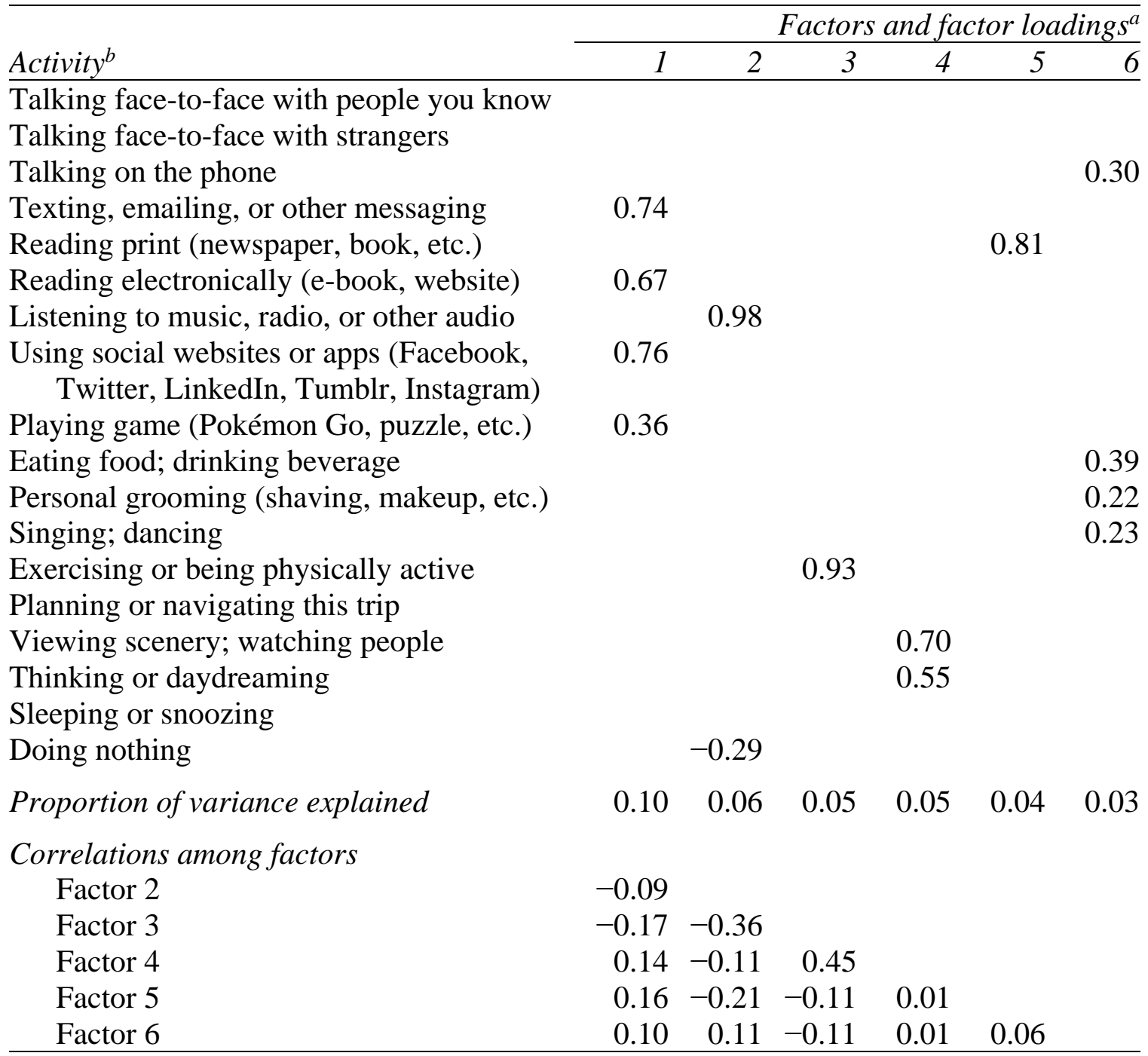

${ }^{a}$ Factor loadings $< \pm 0.20$ are not shown.

b The activities "Writing or editing paper documents," "Writing or editing electronic documents," "Watching movie, TV, or other video," "Smoking or vaping," and "Caring for children or pets (dog walking, etc.)" were not included due to low response frequencies $(<15)$.

Next, binary logit models of activity participation were estimated for each of the remaining 13 unique activities and each of the two new activity groups constructed from the EFA. Studies going beyond bivariate analyses have also predicted activity participation 
using binary logit models (Berliner et al., 2015; Guo et al., 2015; Russell et al., 2011; Timmermans \& van der Waerden, 2008; van der Waerden et al., 2009) or skewed logit (scobit) models (Zhang \& Timmermans, 2010).

After soliciting responses on activity participation and (percentage) duration, the survey asked about travel usefulness: "In terms of its value to you, overall, how useful would you rate the time you spent commuting? Ignore the value of getting to your destination, and think only about the things you did while commuting and the time you spent doing them.” This language was borrowed from Circella et al. (2015). Responses were on a five-point Likert-type scale: "Mostly wasted; Somewhat wasted; Neither wasted nor useful; Somewhat useful; Mostly useful."

Given that the travel usefulness question was measured on an ordinal scale and that responses were not normally distributed (negatively skewed and platykurtic), an ordered logit model of travel usefulness was estimated. Other studies have used multinomial logit (Susilo et al., 2012) or ordered probit (Circella et al., 2015) models to predict travel usefulness. Ordered logit and ordered probit models usually yield similar results with respect to tests of parameter significance. Model estimation was conducted using the MASS (Venables \& Ripley, 2002) and stats packages in R.

Independent variables in all of the models included trip characteristics (commute mode, travel time, number of cotravelers), weather (temperature, precipitation), traveler demographics and socioeconomics (individual, household, transportation, and job attributes), and traveler perceptions (satisfaction with typical commute travel time, selfreported ideal commute travel time, and the teleportation test). The selected variables have been used in other travel-based multitasking studies and/or were available for at least a 
subsample of the dataset. The traveler perception questions have been occasionally used in previous research investigating the positive utility of travel (Russell \& Mokhtarian, 2015). The travel usefulness models added activity participation and an ordinal measure of travel liking (Ory \& Mokhtarian, 2005). Before the independent variables entered the model, they were examined for multicollinearity issues; variables that were moderately-to-strongly correlated (>0.40) were removed. See Table 4.2 for a full list of the independent variables and their descriptive statistics.

\section{Table 4.2 Descriptive statistics}

\begin{tabular}{lrrrr}
\hline & \multicolumn{3}{c}{ Categorical } & \multicolumn{2}{c}{ Continuous } \\
\cline { 2 - 5 } Variable & $\#$ & $\%$ & Mean & $S D$ \\
\hline Trip characteristics & 30 & 4.3 & & \\
Mode: Walk & 114 & 16.5 & & \\
Bicycle & 175 & 25.4 & & \\
Transit & 35 & 5.1 & & \\
Auto, passenger & 336 & 48.7 & & \\
Auto, driver & & & 35.66 & 21.27 \\
Travel time (minutes) & & & 0.24 & 0.70 \\
\# cotravelers & 155 & 22.9 & & \\
Temperature $\left({ }^{\circ}\right.$ F) $\Delta$ from average & & & & \\
Day precipitation $\geq 0.10$ in & 142 & 19.4 & & \\
Traveler socio-demographics & 190 & 25.9 & & \\
Age: 18-34 years & 174 & 23.7 & & \\
35-44 years & 48 & 6.5 & & \\
55-64 years & 403 & 55.4 & & \\
65+ years & 24 & 3.3 & & \\
Gender: Female & 101 & 13.7 & & \\
Race/ethnicity: Missing & 54 & 7.3 & & \\
Hispanic/non-white/multiple & 54 & 7.3 & & \\
Disability & 131 & 17.9 & & \\
Student & 318 & 43.4 & & \\
Education: No college degree & & & 0.41 & 0.81 \\
$\quad$ Graduate degree & & & 0.51 & 0.71 \\
\# children (age $\leq 16)$ & & & \\
\# workers & & &
\end{tabular}




\begin{tabular}{|c|c|c|c|c|c|}
\hline \multirow{2}{*}{ Variable } & & \multicolumn{2}{|c|}{ Categorical } & \multicolumn{2}{|c|}{ Continuou: } \\
\hline & & \# & $\%$ & Mean & $S D$ \\
\hline \multicolumn{2}{|c|}{ \# seniors (age 65+) } & & & 0.06 & 0.28 \\
\hline \multirow{5}{*}{ Income: } & & 64 & 8.7 & & \\
\hline & & 125 & 17.0 & & \\
\hline & $150 \mathrm{k}$ & 196 & 26.6 & & \\
\hline & & 135 & 18.3 & & \\
\hline & & 55 & 7.5 & & \\
\hline \multicolumn{2}{|c|}{ Multifamily home } & 148 & 20.6 & & \\
\hline \multicolumn{2}{|c|}{ Lived in home: $0-5$ years } & 306 & 42.6 & & \\
\hline \multirow{2}{*}{\multicolumn{2}{|c|}{ \# cars }} & & & 1.74 & 1.03 \\
\hline \# bicycles & & & & 2.46 & 2.03 \\
\hline \multicolumn{2}{|c|}{ Car-share member } & 173 & 23.8 & & \\
\hline \multicolumn{2}{|c|}{ Bike-share member } & 70 & 9.6 & & \\
\hline \multicolumn{2}{|c|}{ Transit pass } & 307 & 42.2 & & \\
\hline \multicolumn{2}{|c|}{ \# commute days } & & & 4.62 & 0.89 \\
\hline \multicolumn{2}{|c|}{ \# hours worked } & & & 42.34 & 10.25 \\
\hline \multicolumn{2}{|c|}{ Flexible work schedule } & 451 & 62.8 & & \\
\hline \multicolumn{2}{|c|}{ Self-employed } & 33 & 4.6 & & \\
\hline \multicolumn{6}{|c|}{ Traveler perceptions } \\
\hline \multirow{2}{*}{\multicolumn{2}{|c|}{$\begin{array}{l}\text { Typical travel time: Dissatisfied } \\
\text { Ideal travel time (minutes) }\end{array}$}} & 239 & 34.2 & & \\
\hline & & & & 13.70 & 8.76 \\
\hline \multicolumn{2}{|c|}{ Teleportation: No } & 261 & 37.5 & & \\
\hline \multicolumn{2}{|c|}{ Travel usefulness: Mostly wasted } & 81 & 11.8 & & \\
\hline \multirow{6}{*}{ Travel liking: } & Somewhat wasted & 128 & 18.7 & & \\
\hline & Somewhat useful & 176 & 25.7 & & \\
\hline & Mostly useful & 157 & 22.9 & & \\
\hline & Disliked & 106 & 15.6 & & \\
\hline & Somewhat liked & 238 & 35.1 & & \\
\hline & Strongly liked & 189 & 27.8 & & \\
\hline
\end{tabular}

\subsection{Results}

\subsubsection{Travel-based multitasking}

Figure 4.1 shows the five most frequently reported activities among commuters using each primary transportation mode. The majority of people walking and bicycling reported exercising or being physically active $(60 \%, 89 \%)$ and engaging in passive 
activities: viewing scenery or watching people $(67 \%, 73 \%)$ and thinking/daydreaming $(63 \%, 60 \%)$. In fact, these two passive activities were also among the top five activities for all modes and were reported by about half of transit riders and auto passengers and about a third of auto drivers. About $80 \%$ of drivers listened to music or the radio while commuting. The next most common activity reported by auto drivers was eating or drinking (33\%). More activities were at least somewhat frequently reported by transit riders and auto passengers than by people walking, bicycling, or driving. Most transit commuters engaged in at least one ICT-based activity: texting, emailing, or messaging (56\%), reading electronically (41\%), and/or using social websites/apps (34\%). Most auto passengers were talking with people they knew (71\%) and listening to audio (66\%); some were also eating or drinking (31\%). In summary, modal differences in travel-based multitasking appear to have loomed large.

\section{Activities (Walking)}

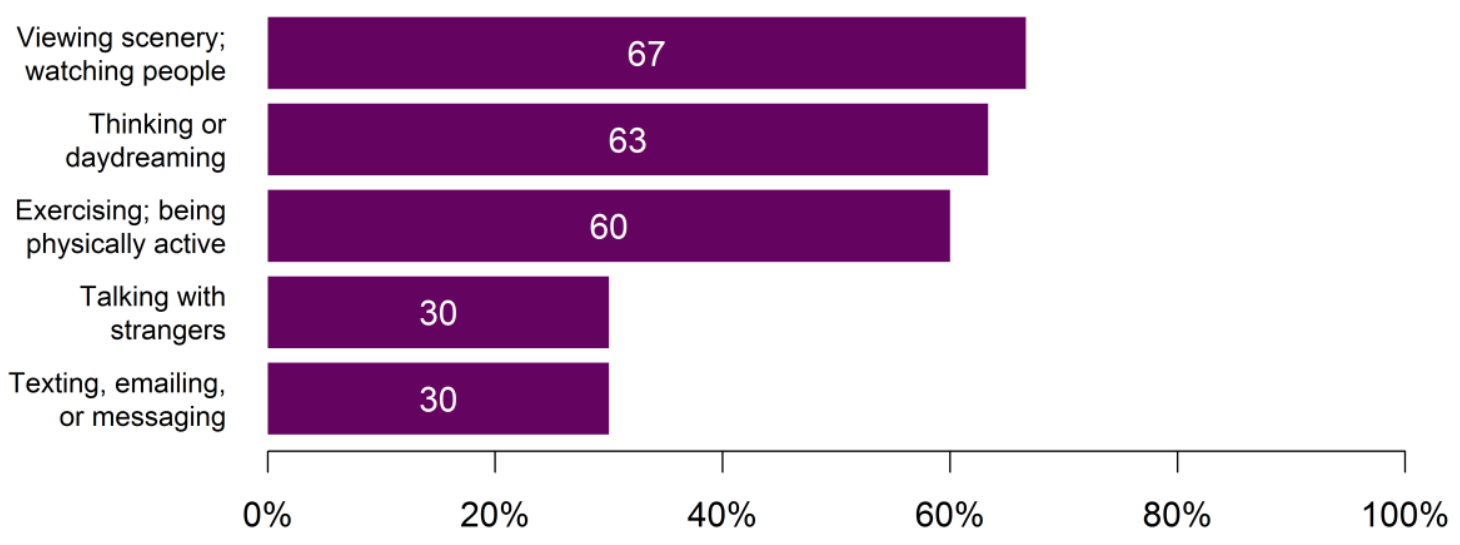




\section{Activities (Bicycling)}

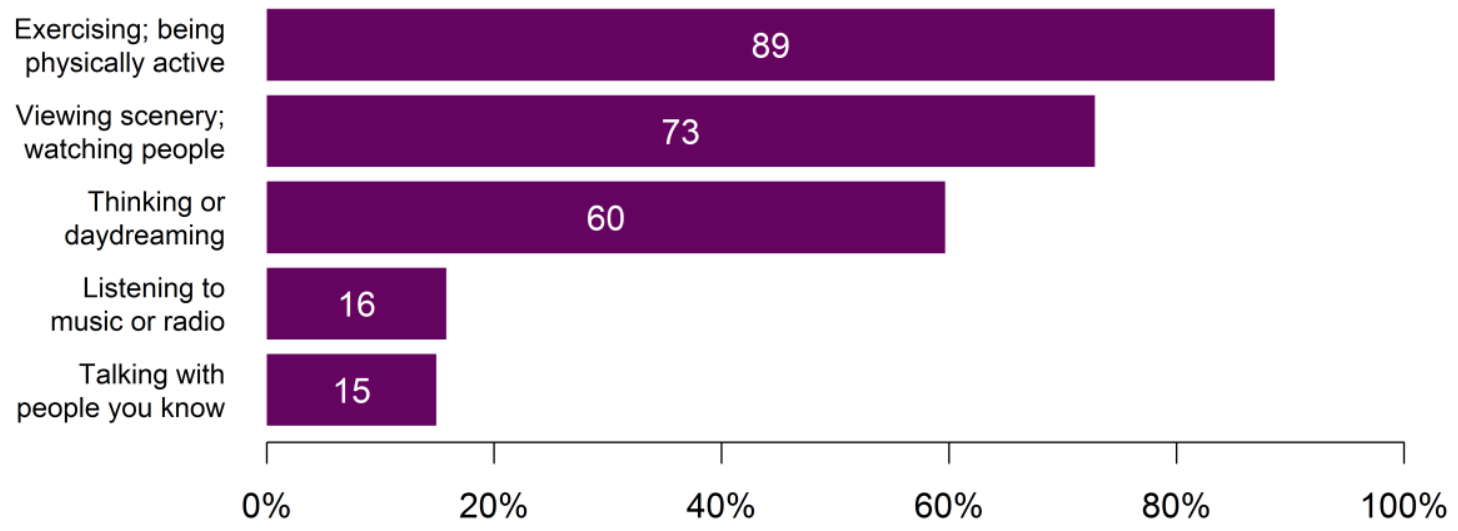

\section{Activities (Transit)}

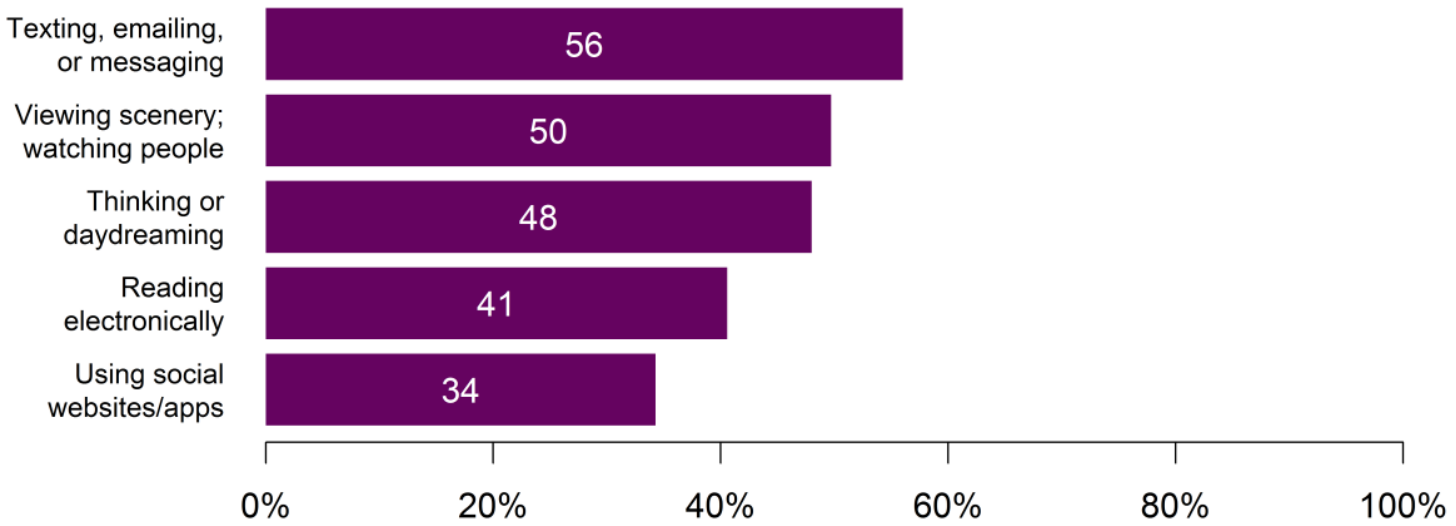

\section{Activities (Auto passenger)}

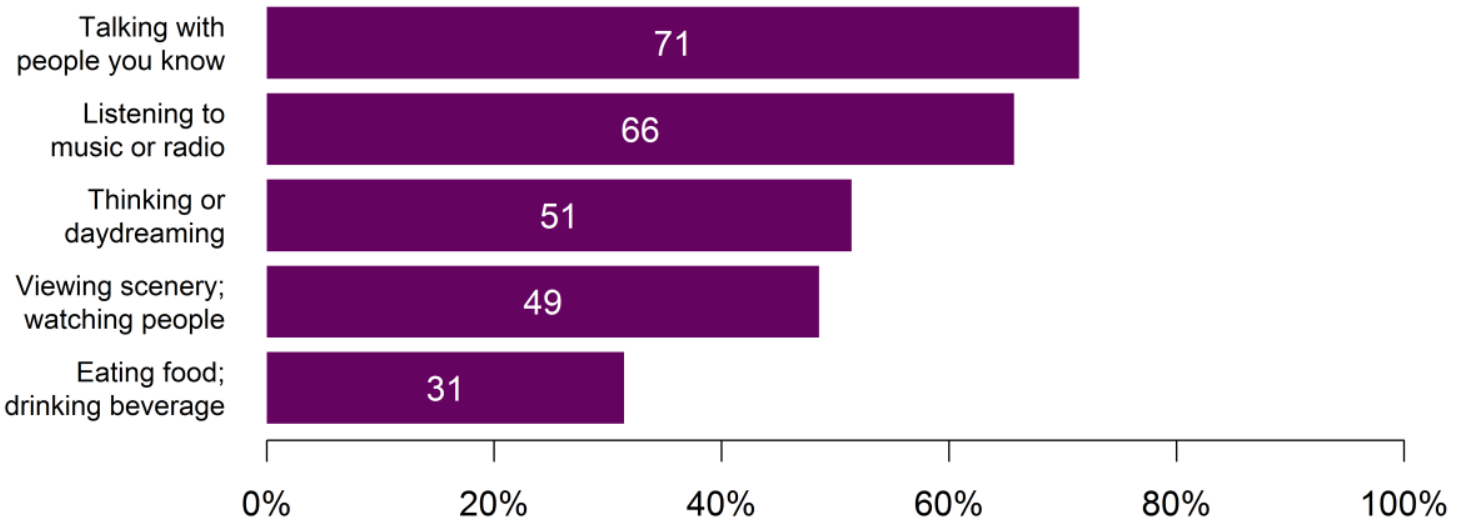




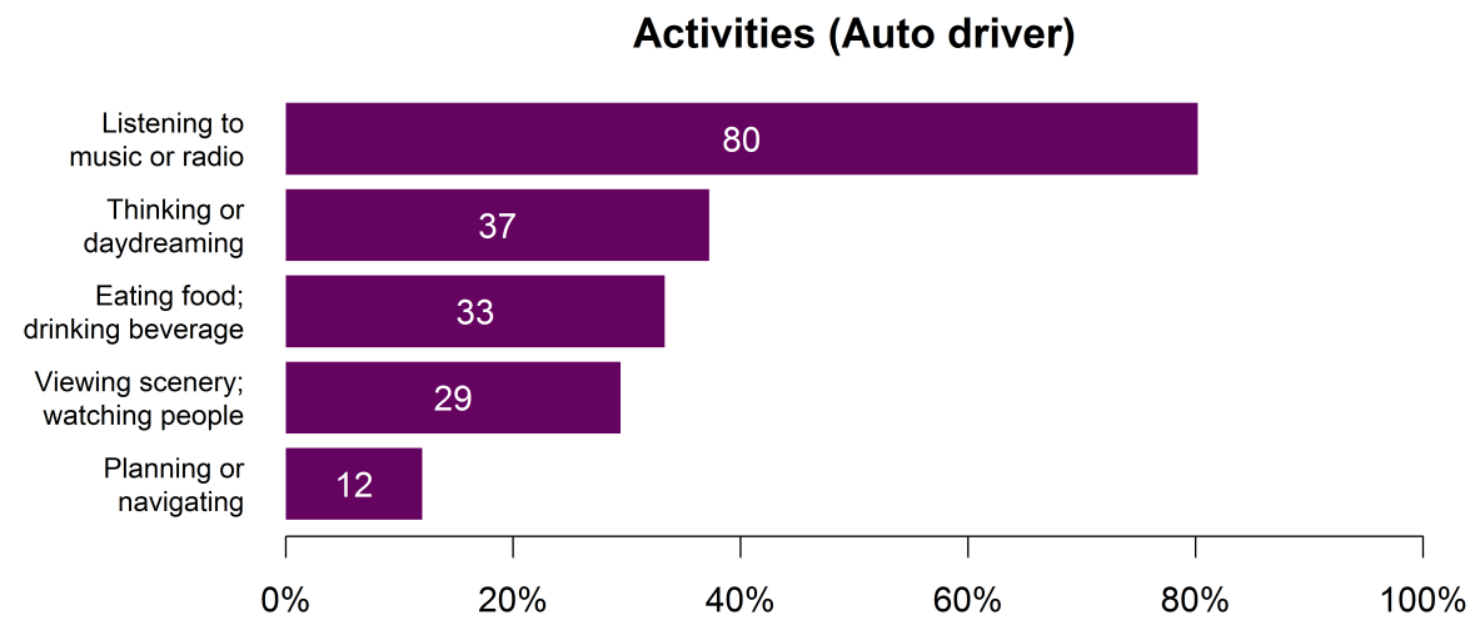

Figure 4.1 Frequently reported activities by commute mode

To understand factors associated with travel-based multitasking, binary logit models were estimated on the multiple categories of activity participation. Model estimation results are shown in Table 4.4 for nine activities and two activity groups: ICT activities (texting/emailing/messaging, reading electronically, using social websites/apps); and Passive activities (viewing scenery or people watching, thinking/daydreaming). Models for game playing, personal grooming, singing/dancing and sleeping/snoozing are not shown because those activities had relatively low response frequencies $(<40)$. Only marginally significant variables $(p \leq 0.10)$ are shown in the table, although all independent variables were included in the models. (Full model results may be obtained by contacting the author.) Table 4.3 shows the number of travelers that reported doing each activity and a goodness-of-fit measure (McFadden's pseudo- $R^{2}$ ) for each binary logit model. 
Table 4.3 Summary statistics of binary logit models of activity participation

\begin{tabular}{lrr}
\hline Model & $\begin{array}{r}\text { \% reporting } \\
\text { the activity }\end{array}$ & $\begin{array}{r}\text { McFadden's } \\
\text { pseudo- } R^{2}\end{array}$ \\
\hline ICT activities & 24.8 & 0.442 \\
Passive activities & 60.1 & 0.117 \\
Talking with people you know & 14.2 & 0.365 \\
Talking with strangers & 8.3 & 0.234 \\
Talking on the phone & 7.1 & 0.255 \\
Reading print & 8.4 & 0.519 \\
Listening to music, radio, audio & 53.1 & 0.328 \\
Eating food; drinking beverage & 22.8 & 0.181 \\
Exercising; being physically active & 18.7 & 0.765 \\
Planning or navigating this trip & 10.0 & 0.174 \\
Doing nothing & 8.7 & 0.100 \\
\hline
\end{tabular}

Goodness-of-fit statistics varied across the models shown. For exercising, the independent variables explained most of the variance in activity participation (0.77). Models for ICT activities, the three talking activities, reading print, and listening to audio had moderate fits, ranging from 0.52 for reading print to 0.23 for talking with strangers. The remaining models had relatively low fits; the independent variables were relatively poor predictors of reports of doing nothing (0.10). 


\section{Table 4.4 Results of binary logit models of activity participation}

\begin{tabular}{|c|c|c|c|c|c|c|c|c|c|}
\hline \multirow{2}{*}{\multicolumn{2}{|c|}{$N=649$}} & \multicolumn{2}{|c|}{ ICT activities } & \multicolumn{2}{|c|}{$\begin{array}{r}\text { Passive } \\
\text { activities }\end{array}$} & \multicolumn{2}{|c|}{$\begin{array}{l}\text { Talking with } \\
\text { people you know }\end{array}$} & \multicolumn{2}{|c|}{$\begin{array}{r}\text { Talking with } \\
\text { strangers }\end{array}$} \\
\hline & & $B$ & $S E$ & $B$ & $S E$ & $B$ & $S E$ & $B$ & $S E$ \\
\hline \multicolumn{10}{|c|}{ Trip characteristics \& weather } \\
\hline \multirow{4}{*}{ Mode: } & Walk & 2.057 & $0.595 *$ & 1.710 & $0.608 *$ & & & 2.996 & $0.729 *$ \\
\hline & Bicycle & $\mathrm{n} / \mathrm{a}$ & & 1.689 & $0.342 *$ & & & 1.600 & $0.654 *$ \\
\hline & Auto, passenger & 1.134 & $0.556 *$ & & & 3.044 & $0.592 *$ & & \\
\hline & Transit & 3.752 & $0.418 *$ & 0.711 & $0.280 *$ & -1.385 & $0.505 *$ & 2.329 & $0.607 *$ \\
\hline \multicolumn{2}{|c|}{ Travel time (minutes) } & & & & & 0.025 & $0.008 *$ & & \\
\hline \multirow{2}{*}{\multicolumn{2}{|c|}{$\begin{array}{l}\text { \# cotravelers } \\
\text { Temperature }\left({ }^{\circ} \mathrm{F}\right) \Delta \text { from average } \\
\text { Day precipitation } \geq 0.10 \text { in }\end{array}$}} & 0.356 & $0.191 \sim$ & & & 1.488 & $0.218 *$ & & \\
\hline & & & & & & & & 0.066 & $0.037 \sim$ \\
\hline \multicolumn{10}{|c|}{ Traveler socio-demographics } \\
\hline \multirow{2}{*}{\multicolumn{10}{|c|}{$\begin{array}{ll}\text { Age: } & 18-34 \text { years } \\
& 35-44 \text { years }\end{array}$}} \\
\hline & & & & & & & & & \\
\hline \multicolumn{2}{|r|}{$55-64$ years } & -0.953 & $0.388 *$ & & & & & & \\
\hline & $65+$ years & -1.620 & $0.652 *$ & & & & & & \\
\hline \multicolumn{10}{|c|}{ Gender: Female } \\
\hline \multicolumn{2}{|c|}{ Race/ethnicity: Missing } & & & & & & & 1.421 & $0.846 \sim$ \\
\hline \multicolumn{2}{|c|}{ Hispanic/non-white/multiple } & & & & & & & -1.404 & $0.773 \sim$ \\
\hline \multirow{2}{*}{\multicolumn{2}{|c|}{$\begin{array}{l}\text { Disability } \\
\text { Student }\end{array}$}} & & & & & & & & \\
\hline & & & & -0.564 & $0.338 \sim$ & -1.422 & $0.767 \sim$ & & \\
\hline \multicolumn{2}{|c|}{ Education: No college degree } & & & & & -0.911 & $0.528 \sim$ & & \\
\hline \multicolumn{10}{|c|}{ Graduate degree } \\
\hline \multicolumn{10}{|c|}{$\#$ children $($ age $\leq 16)$} \\
\hline \multicolumn{10}{|c|}{ \# workers } \\
\hline \multicolumn{10}{|c|}{ \# seniors (age 65+) } \\
\hline \multirow[t]{5}{*}{ Income: } & : $\$ 0-50 \mathrm{k}$ & & & & & & & & \\
\hline & $\$ 50-75 \mathrm{k}$ & & & 0.707 & $0.301 *$ & 1.163 & $0.470 *$ & & \\
\hline & $\$ 100-150 \mathrm{k}$ & & & & & & & & \\
\hline & $\$ 150 \mathrm{k}+$ & & & & & & & & \\
\hline & Missing & & & & & & & & \\
\hline Multifar & mily home & & & 0.465 & $0.275 \sim$ & & & & \\
\hline $\begin{array}{l}\text { Lived in } \\
\text { \# cars }\end{array}$ & home: $0-5$ years & 0.553 & $0.318 \sim$ & & & & & & \\
\hline \# bicycl & & & & & & & & 0.228 & $0.094 *$ \\
\hline Car-sha & re member & 0.873 & $0.322 *$ & & & & & & \\
\hline Bike-sh & are member & & & & & & & & \\
\hline Transit & pass & & & -0.395 & $0.218 \sim$ & & & & \\
\hline \# comm & lute days & & & -0.239 & $0.125 \sim$ & & & & \\
\hline \# hours & worked & & & -0.023 & $0.012 \sim$ & & & & \\
\hline Flexible & work schedule & & & & & & & & \\
\hline Self-em & iployed & & & & & & & & \\
\hline Traveler per & rceptions & & & & & & & & \\
\hline Typical & travel time: Dissatisfied & 0.733 & $0.305 *$ & & & -0.979 & $0.383 *$ & & \\
\hline Ideal tra & avel time (minutes) & & & & & -0.045 & $0.021 *$ & & \\
\hline Telepor & tation: No & & & & & & & & \\
\hline Intercept & & -3.709 & $1.190 *$ & 1.997 & $0.864 *$ & -3.925 & $1.490 *$ & -4.437 & $1.528 *$ \\
\hline
\end{tabular}

Statistical significance: $*=p \leq 0.05, \sim=p \leq 0.10$. Coefficients with $p>0.10$ not shown.

Note: $\mathrm{n} / \mathrm{a}=$ no activity participation observed for this mode. 


\begin{tabular}{|c|c|c|c|c|c|c|c|c|c|}
\hline \multirow{2}{*}{\multicolumn{2}{|c|}{$N=649$}} & \multicolumn{2}{|c|}{$\begin{array}{r}\text { Talking on the } \\
\text { phone }\end{array}$} & \multicolumn{2}{|c|}{ Reading print } & \multicolumn{2}{|c|}{$\begin{array}{l}\text { Listening to } \\
\text { music, radio }\end{array}$} & \multicolumn{2}{|c|}{$\begin{array}{l}\text { Eating food; } \\
\text { drinking }\end{array}$} \\
\hline & & $B$ & $S E$ & $B$ & $S E$ & $B$ & $S E$ & $B$ & SE \\
\hline \multicolumn{10}{|c|}{ Trip characteristics \& weather } \\
\hline \multirow{4}{*}{ Mode: } & Walk & & & $\mathrm{n} / \mathrm{a}$ & & -2.992 & $0.561 *$ & -1.777 & $0.619 *$ \\
\hline & Bicycle & -2.344 & $1.107 *$ & & & -3.305 & $0.384 *$ & -2.859 & $0.547 *$ \\
\hline & Auto, passenger & & & $\mathrm{n} / \mathrm{a}$ & & & & & \\
\hline & Transit & -1.192 & $0.626 \sim$ & & & -2.780 & $0.337 *$ & -2.215 & $0.371 *$ \\
\hline \multicolumn{2}{|c|}{ Travel time (minutes) } & 0.024 & $0.009 *$ & & & 0.013 & $0.006 *$ & 0.022 & $0.006 *$ \\
\hline \multicolumn{2}{|c|}{ \# cotravelers } & & & & & -0.393 & $0.163 *$ & & \\
\hline \multicolumn{2}{|c|}{ Temperature $\left({ }^{\circ} \mathrm{F}\right) \Delta$ from average } & \multicolumn{7}{|c|}{ Day precipitation $\geq 0.10$ in } & $0.022 \sim$ \\
\hline \multicolumn{10}{|c|}{ Traveler socio-demographics } \\
\hline \multirow[t]{4}{*}{ Age: } & $18-34$ years & & & 1.890 & $0.797 *$ & & & & \\
\hline & $35-44$ years & & & & & 0.677 & $0.314 *$ & & \\
\hline & $55-64$ years & & & & & & & & \\
\hline & $65+$ years & & & & & & & & \\
\hline \multicolumn{2}{|c|}{ Gender: Female } & & & & & & & 0.532 & $0.239 *$ \\
\hline \multicolumn{10}{|c|}{ Race/ethnicity: Missing } \\
\hline \multicolumn{10}{|c|}{ Hispanic/non-white/multiple } \\
\hline \multicolumn{2}{|c|}{ Disability } & & & & & & & & \\
\hline \multicolumn{2}{|c|}{ Student } & 1.060 & $0.559 \sim$ & -1.586 & $0.963 \sim$ & & & -0.764 & $0.457 \sim$ \\
\hline \multicolumn{2}{|c|}{ Graduate degree } & & & & & 0.525 & $0.319 \sim$ & & \\
\hline \multirow{2}{*}{\multicolumn{2}{|c|}{$\begin{array}{l}\text { \# children (age } \leq 16) \\
\text { \# workers }\end{array}$}} & & & & & & & -0.303 & $0.154 *$ \\
\hline & & & & & & & & & \\
\hline \multicolumn{2}{|c|}{ \# seniors (age $65+$ ) } & & & & & & & & \\
\hline \multirow[t]{5}{*}{ Income: } & $\$ 0-50 \mathrm{k}$ & 1.766 & $0.858 *$ & & & & & & \\
\hline & $\$ 50-75 \mathrm{k}$ & 1.648 & $0.758 *$ & & & & & & \\
\hline & $\$ 100-150 \mathrm{k}$ & 1.388 & $0.711 \sim$ & & & & & & \\
\hline & $\$ 150 \mathrm{k}+$ & 1.714 & $0.718 *$ & & & & & & \\
\hline & Missing & 1.848 & $0.910 *$ & & & & & & \\
\hline Multifa & mily home & & & & & & & & \\
\hline Lived ir & $n$ home: $0-5$ years & & & -1.007 & $0.579 \sim$ & & & & \\
\hline \# cars & & & & 0.603 & $0.298 *$ & & & & \\
\hline \# bicycl & & & & & & & & 0.107 & $0.065 \sim$ \\
\hline Car-sha & re member & & & & & -0.719 & $0.275 *$ & & \\
\hline Bike-sh & are member & & & & & & & & \\
\hline Transit & pass & & & & & & & & \\
\hline \# comm & hute days & & & & & & & & \\
\hline \# hours & worked & 0.059 & $0.020 *$ & & & & & & \\
\hline $\begin{array}{l}\text { Flexible } \\
\text { Self_em }\end{array}$ & work schedule & & & & & & & -0.435 & $0.227 \sim$ \\
\hline Self-em & iployed & & & & & & & & \\
\hline Traveler per & rceptions & & & & & & & & \\
\hline Typical & travel time: Dissatisfied & & & & & 0.620 & $0.242 *$ & & \\
\hline Ideal tre & avel time (minutes) & & & & & & & & \\
\hline Telepor & tation: No & & & & & & & & \\
\hline Intercept & & -8.055 & $1.702 *$ & & & 2.078 & $0.977 *$ & & \\
\hline
\end{tabular}

Statistical significance: $*=p \leq 0.05, \sim=p \leq 0.10$. Coefficients with $p>0.10$ not shown.

Note: $\mathrm{n} / \mathrm{a}=$ no activity participation observed for this mode. 


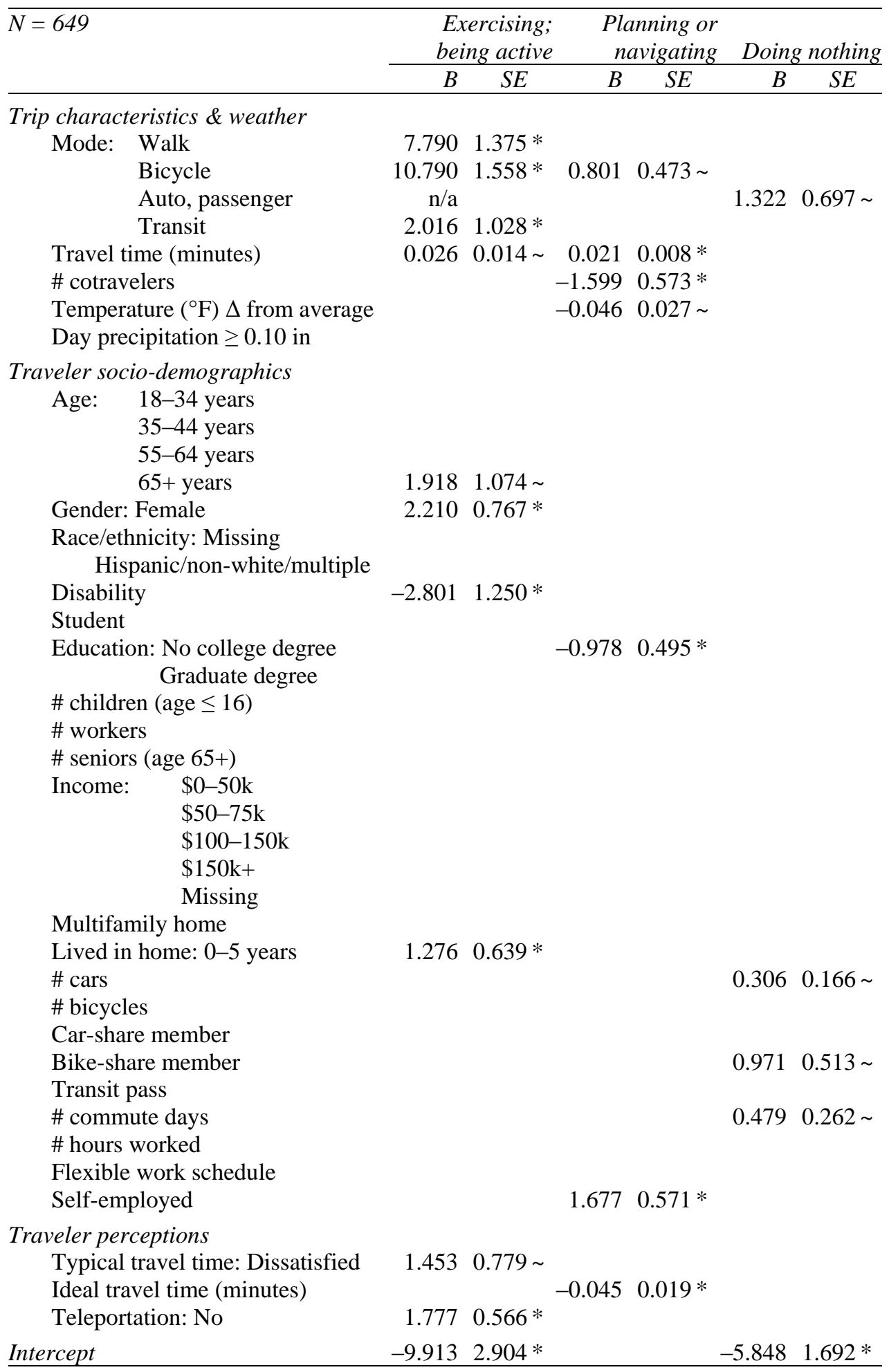

Statistical significance: $*=p \leq 0.05, \sim=p \leq 0.10$. Coefficients with $p>0.10$ not shown.

Note: $\mathrm{n} / \mathrm{a}=$ no activity participation observed for this mode. 
Participation in at least one ICT activity (texting/emailing/messaging, reading electronically, using social websites/apps) was reported by about $25 \%$ of commuters. Travel mode was a significant factor in this model: Transit riders were most likely to participate in ICT activities, followed by people walking and then by auto passengers. Older people were less likely to engage in these technology-based activities while traveling. ICT activity participation was positively associated with the number of cotravelers on the trip and having a car-share membership. Those who were dissatisfied with their typical commute travel time were also more likely to report doing these activities.

More than half of the sample (60\%) participated in passive activities (viewing scenery or people watching, thinking/daydreaming). Again, commute mode was a significant predictor in this model: Nonauto travelers were more likely to report these passive activities than auto drivers or passengers. People holding a transit pass or working more hours or commuting more days per week were less likely to do these activities. Passive activity participation was more common among commuters in middle household incomes $(\$ 50,000-\$ 75,000)$.

Results differed within the three models of verbal communication activities. Almost twice as many people reported talking with people they knew (14\%) than reported talking with strangers $(8 \%)$ or on the phone $(7 \%)$. Nonauto travelers were significantly more likely to talk to strangers than auto commuters; transit commuters were the least likely to talk face-to-face with family, friends, or acquaintances; and bicycle commuters were least likely to talk on the phone. Auto passengers were more likely to talk with people they knew, as were commuters traveling with other people. Travel time was a positive factor, but not for talking with strangers. Various other traveler demographic and 
socioeconomic characteristics were significant. For instance, lower- and higher-income travelers were more likely to talk on the phone than middle-income commuters. Regarding traveler perceptions, commuters who were satisfied with their typical travel time and who desired shorter commutes were more likely to talk with people they knew.

Listening to music, radio, or other audio was the second most commonly reported activity (53\%), and was much more likely to be reported by auto drivers or passengers. Younger people and those traveling alone were more likely to listen to audio, while deviations from average temperatures made listening less likely. This activity was more common on longer trips and for commuters who were dissatisfied with their typical travel time. Listening to audio was also negatively associated with having a college degree and being a car-share member.

As the best fitting model, exercising or being physically active (19\% of travelers) was best predicted by commute mode: It was reported by most walk and bicycle commuters, some transit commuters, and almost no auto commuters. Exercise was more likely on longer duration commutes. Women, older adults, and new residents (who moved within the last five years) were more likely to report exercising, while people with disabilities were less likely to have physically active commutes. Two traveler perceptions were significant: People who were dissatisfied with their typical travel times or who preferred not to teleport were more likely to exercise while commuting.

A quick summary of the remaining models follows. Reading printed books or newspapers was done almost exclusively by transit commuters. Surprisingly, younger travelers were more likely to read print, as were those owning more cars. Auto commuters were most likely to eat or drink on the go, as were women and those with longer travel 
times; those with a flexible work schedule and more children at home were less likely to eat and drink. For planning and navigation activities, bicycling and travel time were positive factors. People traveling alone and those with college degrees were also more likely to report spending time navigating or planning the trip. Few variables were significant predictors of the classic antiactivity, doing nothing. Auto passengers were more likely to report doing nothing, as were people with more cars, with a bike-share membership, or who commute more frequently.

\subsubsection{Travel usefulness}

Figure 3.7 displays responses to the travel usefulness questions, summarized by commute mode. As described in Chapter 3, significant modal differences in travel usefulness were reported: most people walking (87\%) and bicycling (94\%) and slightly

more than half of transit commuters $(57 \%)$ and auto passengers $(54 \%)$ had at least somewhat useful commutes; only about half of auto drivers felt like their commuters were at least somewhat wasted time $(50 \%)$.

To understand factors associated with ratings of travel usefulness, several ordered logit models were estimated using a variety of specifications. Model estimation results are shown in Table 4.5 for two different models: the primary model using activity participation (yes vs. no), and an alternate model using activity duration (minutes). The activity duration model is shown because it offers different interpretations of travel time and activity engagement during travel than the activity participation model. The dependent variable in both models is the ordered categorical travel usefulness variable, with "Mostly useful" as the most positive category. All traveler characteristics variables shown in Table 4.2 were 
included in the models, but only marginally significant variables $(p \leq 0.10)$ are shown.

(Full model results are available from the author.)

Table 4.5 Results of ordered logit models of travel usefulness

\begin{tabular}{|c|c|c|c|c|c|c|c|}
\hline \multirow{2}{*}{ Variable } & & \multicolumn{3}{|c|}{$\begin{array}{r}\text { Model with } \\
\text { activity participation }\end{array}$} & \multicolumn{3}{|c|}{$\begin{array}{r}\text { Model with } \\
\text { activity duration }\end{array}$} \\
\hline & & $B$ & $S E$ & $p$ & $B$ & $S E$ & $p$ \\
\hline \multicolumn{8}{|c|}{ Trip characteristics \& weather } \\
\hline \multirow{2}{*}{\multicolumn{2}{|c|}{$\begin{array}{ll}\text { Mode: } & \text { Walk } \\
& \text { Bicycle }\end{array}$}} & 1.024 & 0.801 & 0.201 & 1.845 & 0.518 & $0.000 *$ \\
\hline & & 2.645 & 0.679 & $0.000 *$ & 2.504 & 0.406 & $0.000 *$ \\
\hline \multicolumn{2}{|c|}{$\begin{array}{l}\text { Bicycle } \\
\text { Auto, passenger }\end{array}$} & 2.451 & 0.784 & $0.002 *$ & 1.698 & 0.448 & $0.000 *$ \\
\hline \multicolumn{2}{|c|}{ Transit } & 1.306 & 0.521 & $0.012 *$ & 1.667 & 0.331 & $0.000 *$ \\
\hline \multicolumn{2}{|c|}{ Travel time (minutes) } & & & & -0.010 & 0.007 & 0.157 \\
\hline \multirow[t]{5}{*}{ Travel time $x$} & Walk & 0.037 & 0.023 & 0.105 & & & \\
\hline & Bicycle & 0.016 & 0.017 & 0.327 & & & \\
\hline & Auto, driver & -0.008 & 0.007 & 0.252 & & & \\
\hline & Auto, passenger & -0.045 & 0.021 & $0.031 *$ & & & \\
\hline & Transit & -0.005 & 0.007 & 0.465 & & & \\
\hline \multicolumn{2}{|c|}{ Day precipitation $\geq 0.10$ in } & -0.285 & 0.191 & 0.136 & -0.352 & 0.196 & $0.073 \sim$ \\
\hline \multicolumn{8}{|c|}{ Travel activities (participation or duration) } \\
\hline \multicolumn{2}{|c|}{ ICT activities } & 0.297 & 0.242 & 0.220 & -0.003 & 0.007 & 0.713 \\
\hline \multicolumn{2}{|c|}{ Passive activities } & -0.219 & 0.175 & 0.211 & -0.007 & 0.005 & 0.118 \\
\hline \multicolumn{2}{|c|}{ Talking with people you know } & 0.346 & 0.281 & 0.219 & 0.009 & 0.012 & 0.435 \\
\hline \multicolumn{2}{|c|}{ Talking with strangers } & -0.023 & 0.314 & 0.942 & -0.053 & 0.061 & 0.383 \\
\hline \multicolumn{2}{|c|}{ Talking on the phone } & -0.216 & 0.319 & 0.499 & -0.002 & 0.021 & 0.937 \\
\hline \multicolumn{2}{|c|}{ Reading print } & 0.374 & 0.326 & 0.252 & 0.019 & 0.012 & 0.115 \\
\hline \multicolumn{2}{|c|}{ Listening to music, radio, audio } & 0.113 & 0.205 & 0.583 & 0.005 & 0.006 & 0.424 \\
\hline \multicolumn{2}{|c|}{ Playing game } & 0.048 & 0.378 & 0.899 & 0.012 & 0.013 & 0.381 \\
\hline \multicolumn{2}{|c|}{ Eating food; drinking beverage } & -0.115 & 0.202 & 0.567 & 0.003 & 0.012 & 0.826 \\
\hline \multicolumn{2}{|c|}{ Personal grooming } & 0.479 & 0.461 & 0.300 & -0.047 & 0.032 & 0.140 \\
\hline \multicolumn{2}{|c|}{ Singing; dancing } & 0.786 & 0.364 & $0.031 *$ & 0.059 & 0.022 & $0.008 *$ \\
\hline \multicolumn{2}{|c|}{ Exercising; being physically active } & -0.104 & 0.431 & 0.809 & 0.032 & 0.011 & $0.005 *$ \\
\hline \multicolumn{2}{|c|}{ Planning or navigating this trip } & -0.557 & 0.268 & $0.038 *$ & -0.007 & 0.016 & 0.674 \\
\hline \multicolumn{2}{|c|}{ Sleeping or snoozing } & -0.238 & 0.492 & 0.629 & 0.007 & 0.017 & 0.663 \\
\hline Doing nothing & & -0.859 & 0.309 & $0.006 *$ & -0.039 & 0.017 & $0.020 *$ \\
\hline Traveler socio-dem & graphics & & & & & & \\
\hline Age: $18-34$ ye & & -0.477 & 0.287 & $0.097 \sim$ & -0.496 & 0.293 & $0.091 \sim$ \\
\hline \# children (age & 16) & 0.180 & 0.109 & $0.099 \sim$ & 0.134 & 0.110 & 0.224 \\
\hline \# bicycles & & -0.081 & 0.048 & $0.091 \sim$ & -0.073 & 0.049 & 0.136 \\
\hline \# commute day & & 0.207 & 0.104 & $0.047 *$ & 0.204 & 0.106 & $0.054 \sim$ \\
\hline Traveler perceptiol & & & & & & & \\
\hline Typical travel & me: Dissatisfied & -0.395 & 0.201 & $0.050 *$ & -0.418 & 0.203 & $0.039 *$ \\
\hline Teleportation: & & 0.515 & 0.177 & $0.004 *$ & 0.595 & 0.178 & $0.001 *$ \\
\hline Travel liking: & Disliked & -0.603 & 0.276 & $0.029 *$ & -0.661 & 0.282 & $0.020 *$ \\
\hline & Somewhat liked & 0.950 & 0.218 & $0.000 *$ & 0.981 & 0.223 & $0.000 *$ \\
\hline & Strongly liked & 2.250 & 0.269 & $0.000 *$ & 2.281 & 0.274 & $0.000 *$ \\
\hline
\end{tabular}




\begin{tabular}{|c|c|c|c|c|c|c|}
\hline \multirow[b]{2}{*}{ Variable } & \multicolumn{3}{|c|}{$\begin{array}{r}\text { Model with } \\
\text { activity participation }\end{array}$} & \multicolumn{3}{|c|}{$\begin{array}{r}\text { Model with } \\
\text { activity duration }\end{array}$} \\
\hline & $B$ & $S E$ & $p$ & $B$ & $S E$ & $p$ \\
\hline \multicolumn{7}{|l|}{ Thresholds } \\
\hline Mostly vs. Somewhat wasted & -0.696 & 0.794 & 0.381 & -0.846 & 0.778 & 0.277 \\
\hline Somewhat wasted vs. Neither & 1.078 & 0.791 & 0.174 & 1.003 & 0.773 & 0.195 \\
\hline Neither vs. Somewhat useful & 2.545 & 0.799 & $0.002 *$ & 2.467 & 0.781 & $0.002 *$ \\
\hline Somewhat vs. Mostly useful & 4.775 & 0.816 & $0.000 *$ & 4.692 & 0.799 & $0.000 *$ \\
\hline \multicolumn{7}{|l|}{ Model fit statistics } \\
\hline Sample size $(N)$ & 642 & & & 619 & & \\
\hline Deviance (thresholds model), $d f$ & $2,021.7$ & 638 & & $1,944.8$ & 615 & \\
\hline Deviance (full model), $d f$ & $1,495.7$ & 575 & & $1,437.5$ & 556 & \\
\hline Likelihood ratio test $\left(\Delta G^{2}\right), \Delta d f, p$ & 526.0 & 63 & $0.000 *$ & 507.3 & 59 & $0.000 *$ \\
\hline McFadden's pseudo- $R^{2}$ & 0.260 & & & 0.261 & & \\
\hline Trip characteristics only & 0.155 & & & 0.145 & & \\
\hline Travel activities only & 0.109 & & & 0.101 & & \\
\hline Socio-demographics only & 0.042 & & & 0.042 & & \\
\hline Perceptions only & 0.162 & & & 0.162 & & \\
\hline
\end{tabular}

Statistical significance: $*=p \leq 0.05, \sim=p \leq 0.10$. Coefficients with $p>0.10$ not shown.

Nonlinear representations of travel time were examined but rejected; a quadratic term was not statistically significant, and adding the natural log of travel time only marginally improved the model fit. Interacting travel time with commute mode in the activity participation model yielded a modest but significant reduction in model deviance $\left(\Delta G^{2}=10.7, \Delta d f=4, p<0.05\right)$; the generic travel time coefficient was negative but not statistically significant. The activity duration model does not include the travel time $\times$ mode interaction because it was not statistically significant $\left(\Delta G^{2}=5.6, \Delta d f=4, p>0.10\right)$. The full models - including trip characteristics, travel activities, and traveler characteristicssignificantly reduced the deviance of their corresponding models containing only category thresholds $\left(\Delta G^{2}=526.0, \Delta d f=63, p<0.001\right.$ for the activity participation model; $\Delta G^{2}=$ 507.3, $\Delta d f=59, p<0.001$ for the activity duration model). McFadden's pseudo- $R^{2}$ goodness-of-fit values were approximately 0.26 for both models. 
Results regarding the association between travel-based multitasking and travel usefulness differed slightly between the two models. In the activity participation model, people who were doing nothing or planning/navigating the trip reported less useful commutes, while those who sung or danced had more useful commutes. No other activities were significantly associated with travel usefulness. In the activity duration model, the effects of doing nothing and singing/dancing (but not planning/navigating) were similarly significant. People spending more time doing nothing or engaged in passive activities (viewing scenery, watching people, thinking, or daydreaming; nearly marginally significant) reported more wasteful commutes. On the other hand, more time spent reading print newspapers or books (nearly marginally significant) and singing or dancing was associated with a more useful commute. Notably, travelers exercising for longer durations found their commutes to be more useful.

Differences between the two models were also found for travel time. In the activity participation model with mode-specific travel times, longer commutes were more wasteful for auto passengers but more useful (although not statistically significantly so) for people walking and bicycling. Using a different specification of the travel time $\times$ mode interaction (not shown) in which mode-specific travel time coefficients reflected differences relative to the auto driver travel time parameter, travel time when walking and bicycling was significantly more useful $(B=0.045, S E=0.024, p<0.05$ for walking; $B=0.025, S E=$ $0.018, p<0.10$ for bicycling) than time spent traveling by auto drivers. In the activity duration model, the generic travel time coefficient was negative but not significant. When this interaction was specified (model not shown), the coefficient for auto drivers was negative and marginally significant, and the positive coefficient for walking approached 
significance. More interestingly, adding this interaction made the coefficient on exercising no longer significant $(B=0.018, S E=0.015, p>0.10)$, suggesting high correlations between times spent walking or bicycling and time spent exercising or being physically active.

After controlling for activity participation, travel time (by mode), and traveler characteristics, some modal differences remained. Bicycle and transit commuters and auto passengers found their commutes to be more useful than did auto drivers. The estimated coefficient for walking was positive in both models, but significant only in the activity duration model. (This coefficient became insignificant when a travel time $\times$ mode interaction was specified.) Commuters traveling on a day with rain reported slightly lower levels of usefulness, although this was not significant in the activity participation model.

Few traveler socio-demographic characteristics were significant predictors of travel usefulness in both models. Younger travelers (aged 18-34) were more likely to report their travel time as being less useful. Those who commuted more frequently and who had access to fewer bicycles at home viewed their travel time as being more useful. Instead, traveler perceptions were more strongly associated with travel usefulness. Travelers who were more satisfied with their typical commute travel time and those who would rather not teleport to work reported more useful commutes. Notably, travel liking and travel usefulness on a recent commute trip were strongly and positively related. 


\subsection{Discussion}

\subsubsection{Travel-based multitasking}

This research contributes to the small but growing literature on multimodal travelbased multitasking. It suggests important modal differences in activity participation, roughly following an active vs. passive distinction in required levels of attention to the traveling task (Circella et al., 2015). For driving modes (bicycle, auto driver), for which the traveler must operate a vehicle, the required mental and physical resources dedicated to the driving task prohibited almost all but the most passive of activities, such as listening to music, looking out the window, or thinking. On the other hand, travelers using riding modes (transit, auto passenger) have the ability to do many more things; this characteristic was reflected by greater participation in a variety of activities, including those with higher resource intensities like ICT activities. Not surprisingly, exercising was nearly the exclusive domain of active modes: walking, bicycling, and — to a lesser extent - transit.

Other modal differences and distinctions are relevant. As in past studies, passive activities—viewing scenery or watching people and thinking/daydreaming-were frequently reported in this study by commuters of all modes, specifically by about twothirds of people walking and bicycling, half of people using riding modes, and a third of auto drivers. Auto passengers, being embedded with auto drivers by definition, shared some common activities with those travelers: listening to audio and eating/drinking. There were also interesting associations between mode and social or communication activities. People using personally exposed modes, including walking, bicycling, and transit (Appleyard \& Ferrell, in press) — in which they may be more likely to interact with people 
they do not know, including other travelers — had a higher odds of talking with strangers than auto commuters. On the other hand, auto passengers and people with more cotravelers were more likely to talk face-to-face with people they knew.

Other findings are consistent with hypotheses from the literature review. When significant, associations between travel time and activity participation were in expected directions. Like in previous studies, longer duration trips saw higher levels of participation in at least some activities. A few traveler demographics were related to activity participation as anticipated. In particular, technology use exhibited age differences: Age was negatively associated with ICT activity engagement and listening to music. As in past studies, women were more likely to talk with other people they knew, although the coefficient was not statistically significant.

Activities related to ICTs were more strongly linked to transit than to any other mode. As shown in Figure 4.1, the three activities constituting the ICT activity grouptexting/emailing/messaging, reading electronically, and using social websites/apps—were all among the five most frequent activities reported by transit commuters. In fact, using coefficients from the models of Table 4.4, the odds of ICT activity participation for transit riders was five times higher than for people walking and 13 times higher than for auto passengers. Additionally, in models (not shown) of each individual ICT activity plus playing games, transit commuters were more likely than all other mode users to report each activity. These findings are consistent with other research (Lyons et al., 2016) suggesting that technological developments in digital communication and internet-connected devices such as tablets and smartphones have transformed the user experience for transit riders, potentially facilitating more productive uses of travel time. The results of this study show 
that people walking and auto passengers are also taking advantage of these digital transformations, although to a lesser extent.

\subsubsection{Travel usefulness}

In addition, this research provides interesting insights into subjective assessments of the usefulness of time spent traveling, a topic receiving increased attention in recent years. Consistent with past studies, about $12 \%$ of commuters viewed their travel time as mostly wasted, while about twice as many (23\%) thought their commutes to be mostly useful. Modal differences in travel usefulness were similar to those found in one previous multimodal study (Circella et al., 2015). Most active mode (walk, bicycle) travelers rated their commutes as useful; around half of riding mode (transit, auto passenger) users had at least somewhat useful commutes; and more than half of auto drivers considered their commutes to be wasted time. These conclusions held even after controlling for travel time, activity participation, and traveler characteristics.

As in previous studies, few traditional traveler attributes were associated with travel usefulness: A model with only socio-demographic characteristics had very low goodnessof-fit $\left(R^{2}=0.04\right)$. As has been found in a California study (Circella et al., 2015), age was a positive factor: Younger travelers were more likely to consider their commutes to be wasted time. The lack of significance of most demographic and socioeconomic traveler characteristics foretells difficulties in predicting how useful people will consider their commutes to be without asking them directly.

Instead, traveler attitudes and perceptions seem more closely tied to subjective valuations of travel time, as has been found in the past (Circella et al., 2015). People who 
were more dissatisfied with their typical commute travel time reported more wasteful commutes, while people who did not want to teleport to work had more useful commutes. The inclusion of these variables in the model raises questions about endogeneity: Are people less satisfied with their typical travel times because they do not make productive use of them? Do people prefer not to teleport because they find some aspects, like activity participation, to be useful? A more complex analysis utilizing (for instance) structural equation modeling could help to illuminate these potential bidirectional effects but is beyond the scope of this chapter.

Despite these valid concerns, there are some reasons to believe that including such perceptions into the model of travel usefulness may be an appropriate choice. First, a large amount of the variation in travel usefulness (roughly $75 \%$ of the thresholds-only model deviance) remained unexplained by the independent variables, suggesting that the association with these perceptions is not strongly deterministic. Second, the perception questions (about travel time satisfaction and the teleportation test) asked specifically about a general condition (a traveler's typical commute), while the travel usefulness question asked about a specific case (a traveler's most recent commute trip to work). The consideration of time precedence (Singleton \& Straits, 2005) suggests that the general condition might cause the specific case but not the other way round. Of course, with crosssectional data, the endogeneity issue may empirically remain. In fact, it may be likely that for a frequently repeated travel behavior like commuting, people have the opportunity to equilibrate their prior perceptions about the usefulness of travel and their satisfaction with travel time such that their perceptions fall more in line with their day-to-day experiences. 
These issues suggest the need to conduct longitudinal studies examining changes in perceptions of travel usefulness.

The travel liking variable was included in the model of travel usefulness to specifically address a concern noted in the literature review: that self-reports of the productive time use benefits of travel-based multitasking may have been confounded with enjoyment of the travel experience, as measured by travel liking. In short, people may have reported a useful commute in part because they liked it. Indeed, the model results provide evidence that this may have been the case: Travel liking was positively and significantly related to travel usefulness. Further, the positive effect of singing or dancing - activities that have perhaps the least instrumental or traditionally productive value to travelerssuggests that travelers may have been conflating travel usefulness with travel enjoyment. Nevertheless, this issue may be somewhat exaggerated. Conflation could have happened in the opposite direction: people may have considered the value of their activity participation when considering how much they liked the trip. This bidirectional effect is perhaps likely, given that the travel usefulness question was asked prior to the travel liking question and prior to all other questions about the travel experience.

\subsubsection{General considerations}

The issues raised in the preceding paragraphs suggest the need for more research on subjective assessments of travel usefulness and travel-based multitasking. Different question wordings or orders could be tested to examine which one best measures the desired construct (the value, worth, or usefulness of activity participation during travel). The finding that most activities did not significantly predict travel usefulness may suggest 
that different kinds of questions are needed, perhaps those asking more about the quality of people's effective time use (Rosenfield \& Zhao, 2016). The questionnaire used in the current study specifically eschewed the term "productive" based on the assumption that people may not consider valid examples of travel-based multitasking or travel time use (e.g., listening to music, reading for pleasure) to be traditionally productive activities: i.e., associated with work or job responsibilities. This assumption could be revisited.

This discussion raises broader but related questions: To what extent is travel-based multitasking valued as a productive use of travel time vs. as a way to make otherwise wasted time more tolerable? How much of a positive utility of travel is travel-based multitasking? The finding that most activities (whether measured by participation or duration) were not associated with travel usefulness suggests that many instances of travelbased multitasking are not considered to be good or productive uses of time, and that the answers to the questions above depend on the activity.

Some activities appear to be useful: People who spent more time reading print books/newspapers considered their time to have been useful. Other activities appear to be more about coping with commuting or reducing the disutility of traveling and less about making productive use of travel time: ICT activities, passive activities, talking with people you know, talking on the phone, listening to music, etc. Participation in many of these activities was more likely on longer duration trips, potentially suggesting that travelers are doing these things to reduce the burden or boredom of commuting. Additionally, the positive association of commuting frequency with travel usefulness suggests that travelers who commute more often may have a greater incentive to make use of their travel time. For other activities, dissatisfaction with typical travel times had an impact: Unable to make 
more desirable changes that would increase their travel time satisfaction, these commuters may be killing time on electronic devices or by listening to music. The nearly significant effects of passive activities on travel usefulness suggests that thinking/daydreaming and looking at scenery and people are not considered useful activities. From a different perspective, time constraints may even force people to schedule certain activities during the commute. The positive associations of auto drivers/passengers and travel time with eating/drinking could be the results of long commutes, congestion, or domestic obligations not affording time in these travelers' schedules for breakfast or coffee before leaving home. On the other hand, travelers with more children were less likely to eat/drink on the commute, suggesting that other factors may be at play.

However, even if commuters are really doing most things just to kill time on the commute, these are still instances of a positive utility of travel. Activity participation presumably makes travel utility more positive, even if it does not completely outweigh the disutilities of travel time, cost, or effort. Even reducing a small amount of the commuting burden by viewing scenery or checking social media presumably means that travelers are still better off than if they had been doing nothing. From a policy perspective, although travel-based multitasking may be unlikely to generate travel, it may instead diminish commuters' incentives for reducing travel (Mokhtarian, 2014). In addition, perhaps travelers truly do benefit from the transition time or time out (Jain \& Lyons, 2008) provided by conducting antiactivities like thinking/daydreaming or sleeping/snoozing, but do not fully acknowledge the usefulness of those activities.

One travel activity that appears to be useful-exercising and being physically active - warrants further discussion. As an activity, exercising is unique because it is so 
highly correlated with active travel modes: Most reports of exercising were among people walking and bicycling, and most walk and bicycle commuters reported being physically active for most of the time. It is difficult to empirically disentangle mode from activity. Thus, the model predicting exercise is also, in some respects, predicting walking and bicycling. This study provides evidence to suggest that exercising is likely considered a useful form of travel-based multitasking. Notably, people walking and bicycling had the most useful commutes. Furthermore, people who preferred not to telecommute (presumably because they valued their commute in some way) were more likely to report exercising. According to the activity duration model of travel usefulness, people spending more time exercising or being physically active were more likely to have useful commutes. While exercising was not significant in the activity participation version, the travel time coefficient for walking was positive and there was a positive residual effect of bicycle mode. As noted in the results section, the high covariance between these factors makes representing both walk/bicycle modes and the exercising activity in a model of travel usefulness more challenging.

An additional (conceptual and empirical) issue with the exercising activity is the difficulty distinguishing the useful benefits of travel-based multitasking from the enjoyable benefits related to travel liking, and distinguishing both types of benefits from the traveling itself (walking, bicycling). In the commuting survey, most people walking and bicycling reported useful commutes but also that they liked their commutes. Chapter 2 discusses some of the arguments for and against classifying exercising as a travel activity instead of a beneficial part of the travel experience. For instance, exercising on the commute can substitute for nontravel physical activity like going to the gym. On the other hand, walking 
and bicycling can be a fun activity or a way to improve physical and mental health. More broadly, exercising and doing other activities (e.g., listening to music, viewing scenery, daydreaming) also can help to facilitate the affective enjoyment of the travel experience. Together, these activities could be contributing to the travel usefulness vs. travel liking conflation discussed earlier. One conceptual solution to the dilemma is to say that the doing (exercising) is the multitasked and useful activity, and that any positive emotions, fulfillment, or liking that may result are part of the travel experience. That said, if it is difficult for scholars to conceptually distinguish the two, how should we expect survey respondents to make such a distinction? Future research should tackle the empirical challenges of measuring and modeling the subjective usefulness of exercising and physical activity.

\subsubsection{Limitations and future work}

There were a number of limitations of this study that could be addressed in future work. The survey itself had a limited scope and could accommodate only a small number of questions about travel-based multitasking and travel usefulness. First, it investigated activity participation only while on home-to-work commute trips. While this design avoided some issues by controlling for the destination activity (work), patterns of travelbased multitasking may be different on work-to-home trips or on trips for nonwork purposes (Keseru et al., 2015). Second, the study measured activity participation for the entire trip, so these analyses could not distinguish between what was done via access or egress modes or while waiting (Mishra, Mokhtarian, \& Widaman, 2015) from what was done while on the primary commute mode. Third, survey length restrictions precluded 
questions about item use (Lyons et al., 2016), the quality of travel time use, and reasons for activity participation (Rosenfield \& Zhao, 2016), all of which would be useful for further analyzing travel-based multitasking, travel usefulness, and relationships between ICTs and travel. Fourth, transit modes were restricted to those present in the Portland region, which meant that longer distance commuters and rail-based transit modes with more productive multitasking amenities (tables, WiFi, etc.) could not be analyzed in this study.

More fundamental research on travel-based multitasking and travel usefulness is needed to address some of the issues discussed above. There are tradeoffs between methods of data collection: Passive field observations can more accurately capture activities that may be forgotten on a survey, but surveys can better measure fundamental activities (rather than item use) and subjective assessments. A mixture of methods could be used to enrich multitasking data collections and to determine the approximate amount of error introduced by having respondents recall activity participation and durations on a survey. Regarding durations, more work is needed to examine whether activity participation or duration (or some other measure of quantity) is more closely linked to the usefulness of travel. Further research on the roles of attitudes and perceptions could illuminate the degree to which travel-based multitasking behavior is self-selected: i.e., whether or not polychronic people are more likely to multitasking while traveling. Empirical challenges with representing exercise and physical activity as travel-based multitasking also remain to be solved. Conducting longitudinal studies of travel activity participation, usefulness, and perceptions could help to solve some of the issues surrounding causality and time precedence. 
At a more conceptual level, research on the fundamental motivations for activity participation during travel is needed, especially studies that put travel-based multitasking into the larger context of daily activity patterns, scheduling, and constraints. If people were prohibited from multitasking during travel, would they shift their current travel activities to other times of the day, forgo conducting those activities, or do them in a different way? Conversely, how would people spend their time traveling if vehicles more greatly facilitated travel-based multitasking? It would be interesting to examine which activities can be shifted onto trips, when this most often occurs, why, and what specific attributes of transportation modes can facilitate or hinder multitasking.

\subsubsection{Implications}

This study offers several implications for transportation design, planning, and policy, especially considering advances in transportation technologies. It demonstrates that people walking and bicycling do indeed view as useful the time they spend exercising while commuting. This finding suggests that interventions to increase the use of these nonmotorized modes could make people healthier and more productive in their time use. The model results also highlight the growing relevance of ICT-based activities for travelers, especially for people riding transit and, to a lesser extent, those walking and riding as auto passengers. Transit agency managers might try to increase ridership on some services by leveraging travelers' proclivities to multitask with on-board or station-area amenities like tray tables, charging stations, and $\mathrm{WiFi}$, or with targeted marketing. In addition, transit passengers may see the biggest benefit from future advances in ICT. 
On the other hand, longer-term technological advancements may instead help auto travelers to make better use of their travel time. Autonomous vehicles (AVs) offer the potential to make traveling much more productive and useful. By removing the need to drive, at least part of the time, autonomous vehicles potentially transform auto drivers into travelers that look and act more like auto passengers or transit riders, engaging in more frequent and varied types of travel-based multitasking: reading, eating a meal, watching a movie, or even sleeping. As a result, simulation studies (e.g., Childress et al., 2015) suggest AVs will reduce values of travel time savings, thus increasing travel demand and potentially vehicle miles traveled, all while reshaping where and how people live and get around. Understanding influences on travel-based multitasking and travel usefulness today yields insights that can be used to better quantify the potential impacts of widespread vehicular automation tomorrow. 


\section{Chapter 5 Travel experiences}

\section{Measures and determinants of subjective well-being from the commute: New evidence from Portland, Oregon}

\subsection{Abstract}

Transportation's relationship with well-being is the subject of increased attention. The "positive utility of travel" concept suggests that positive emotions and/or symbolic motivations expressed through the experience of traveling might influence or motivate travel behaviors. Policymakers attempt to improve the health and well-being of populations through interventions to improve transportation experiences and promote healthy and sustainable transport modes, while researchers seek valid and reliable measures of subjective well-being (SWB) in the travel domain in order to study these connections. Unfortunately, most existing psychological measures of SWB are difficult to adapt or have not been tested with respect to travel, specifically. Using the results of a survey of nearly 700 commuters in the Portland, Oregon, area, this study first documents improved measures and then investigates potential determinants of several aspects of SWB in the travel domain: travel affect, travel eudaimonia, and overall hedonic travel well-being.

Using exploratory and confirmatory factor analyses, new measurement models of travel affect (distress, fear, attentiveness, and enjoyment) and travel eudaimonia (protection, freedom, confidence, belonging, and health) were developed, and an existing instrument — the Satisfaction with Travel Scale — was validated. With further testing and validation, these scales could be useful tools for measuring different dimensions of travel SWB in future studies. Models predicting the latent variable constructs as a function of trip 
and traveler characteristics yielded valuable behavioral and psychological insights. Walking and bicycling rated much higher on measures of physical and mental health, confidence, positive affect, and overall hedonic well-being, suggesting significant benefits of physically active commutes. Enhancing the quality of the experience of traveling by various modes - making bicycling feel safer, riding transit more engaging, and driving less stressful—could also significantly improve commuters' well-being.

\subsection{Introduction}

The relationship between transportation and the health and well-being of a population has been the focus of increased attention in the research community. A number of recent reviews of the well-being concept, interpreted through a transportation lens, have appeared in transport journals (Delbosc, 2012; De Vos et al., 2013; Reardon \& Abdallah, 2013; Nordbakke \& Schwanen, 2014). Most reviews of the travel-SWB relationship focus on system-wide mechanisms by which transportation can affect well-being (Delbosc, 2012; Reardon \& Abdallah, 2013): through the economy, the environment, social relationships, and — especially via transportation systems and infrastructure - individuals' mobility (the ability to move) and accessibility (the ability to reach destinations).

This trend follows a broader interest in using well-being concepts and metrics for policymaking (Diener et al., 2009). In recent years, happiness, social capital, and wellbeing have been proposed as alternative goals to economic wealth for governments-e.g., "gross national happiness" over gross national product (Bates, 2009)—and as alternative metrics to utility for individual benefits (Delbosc, 2012; Reardon \& Abdallah, 2013). There is also increased awareness at a more local level of ways to incorporate well-being and 
especially health considerations into transportation planning and policymaking (Singleton $\&$ Clifton, 2017). These activities reflect an underlying push away from economic and towards more fundamental public policy objectives: improving the experiences and lives of a population.

At a more individual level, applications of psychological approaches to well-being within the travel behavior field have grown in number. Subjective well-being (SWB) is a conceptualization of well-being interpreted through the lens of an individual's perceptions and experiences. De Vos et al. (2013) proposed five ways in which travel can affect SWB: “through experiences during (destination-oriented) travel, activity participation enabled by travel, activities during (destination-oriented) travel, trips where travel is the activity, and through potential travel (or motility)" (p. 421). Mokhtarian (in progress) has reinterpreted and characterized these five influences according to their degree of influence on different dimensions of SWB, their application to short-term trip-specific versus long-term general SWB, and the directness of their influence.

While most research has considered transportation's effects on well-being, a pathway in the reverse direction is also possible and likely: Concerns or expectations regarding travel-related well-being could affect travel decisions or motivate certain travel behaviors. Perhaps people make travel decisions in order to increase their well-being (Abou-Zeid \& Ben-Akiva, 2012); this is certainly the premise of utility maximization theory. These issues and challenges related to studying the directionality of causation in the travel-well-being relationship have been noted (Mokhtarian, in progress). De Vos et al. (2013) acknowledge this bidirectional relationship, although they suggest that people 
may act more instinctively than consciously and may "settle for acceptable rather than optimal outcomes" (p. 436) with respect to maximizing travel SWB.

The well-being concept also ties directly into broader issues in the travel behavior field, including the positive utility of travel (PUT) concept (Mokhtarian \& Salomon, 2001; Salomon \& Mokhtarian, 1998). This idea suggests that there can be benefits to be gained from the act or experience of traveling itself, and that some travel may be motivated more by obtaining these benefits than by reaching a destination. The PUT concept is at odds with travel behavior axioms and assumptions of traditional transportation analysis methods, which presume that travel demand is derived from the demand for activities at destinations separated in space, and that travel time is a disutility to be minimized. A growing body of evidence suggests that PUT-related considerations may have at least a modest impact on travel behavior (see Chapter 2).

The aspect of PUT with the greatest relevance for the transportation-SWB relationship revolves around how travel can provide benefits through positive travel experiences. (A second aspect includes travel-based multitasking: making use of one's travel time for other activities.) Everything tied up in the experience of traveling could combine to generate positive emotions (affect) and/or a higher-level sense of satisfaction or fulfillment (eudaimonia). For instance, someone might go out of her/his way to travel on a more scenic route. People might feel happy to view fall leaves or spring flowers, or they may be excited to experience the first snowflake of winter. Some people may purchase and use sports cars to express social status or to feel powerful and in control. For others, riding the bus or bicycling can be, in part, an expression of their environmental values. Workers may enjoy their commutes as time to prepare for or to relax and recover from the 
stresses of work or home responsibilities. As these examples make clear, different travel modes and environments seem to directly influence travel SWB, and prospective considerations of these SWB factors could influence or motivate travel choices and behaviors.

In examining the relationship between transportation and SWB and studying the travel experience aspects of PUT, a number of research challenges emerge. Notably, most psychological instruments used to measure SWB either look at SWB only for life overall or investigate only one dimension. SWB scales have rarely been applied to the travel domain, for good reason — most operate at a different temporal scale or topical focus—and questions remain about whether or not they are well-designed for this purpose. One exception is the Satisfaction with Travel Scale (Ettema et al., 2011), although it too has been used in only a handful of situations. Creating valid and reliable measures of SWB specific to transportation would be a boon for travel behavior research. The analyses presented in this chapter furthers this work.

Research in this area can also add to the field's understanding of travel behavior and contribute to transportation policy discussions. Self-reported assessments of SWB fit into a broader conversation about the use of attitudes and perceptions in travel behavior analysis (Gärling, Gillholm, \& Gärling, 1998; Golob, 2003). While these psychosocial factors may be more closely linked to travel behaviors than objective attributes of tripmakers, they are also more difficult to measure, forecast, and use in a planning or policy framework. If socio-demographic traveler characteristics that are consistently and significantly associated with SWB or other perceptual attributes exist, these objective 
measures could substitute for more subjective measures in travel demand models or other analysis tools. This study takes up these challenges.

\subsubsection{Research questions}

Several research questions surrounding the empirical measurement of SWB during and as an immediate result of travel are addressed in this study: Is the Satisfaction with Travel Scale (STS) a valid measure of SWB in the travel domain in a U.S. context? Is a 2factor or a 3-factor structure for the STS better? Are existing psychological instruments sufficient for measuring travel well-being? Alternatively, is there a better way to measure hedonic and (especially) eudaimonic SWB from travel? Another arm of this investigation looks to identify potential determinants of travel well-being. Specifically: What traveler and trip characteristics are associated with measures of SWB in the travel domain?

The answers to these questions are examined through the analysis of a 2016 survey of commuters in Portland, Oregon. This chapter is structured as follows: First, literature on SWB, the STS, and the affective and eudaimonic aspects of travel well-being is reviewed. Next, the data and methods are summarized. The results of a multistage analysis (descriptive statistics, factor analysis, and regression) are then presented for each facet of travel well-being: the STS, travel affect, and travel eudaimonia. Finally, these results are discussed, including implications for travel SWB measurement, travel behavior knowledge, and policymaking, as well as opportunities for future work. 


\subsection{Literature review}

\subsubsection{Subjective well-being}

The concept of well-being encompasses many aspects, including satisfaction, happiness, health, and quality of life. As a broad topic, well-being's definition and measurement differ across disciplines, generating challenges when applying well-being concepts to travel analysis. Nordbakke and Schwanen (2014) have classified approaches to well-being according to three dimensions: whether it is defined subjectively or objectively; whether it includes hedonic and/or eudaimonic aspects; and whether it is universal or contextual. For example, the economics field is typically concerned with utility, which is a subjective, hedonic, and universalist notion of well-being (based on the satisfaction of stable individual preferences). Subjective perspectives of well-being may be most applicable to the travel behavior field, rooted as it is in economic paradigms and earlier developments in mathematical psychology (McFadden, 2001b).

Subjective well-being (SWB) is typically classified into hedonic and eudaimonic aspects. Hedonic SWB is related to utility, the satisfaction of one's preferences, mood, and feelings of pleasure and happiness, while eudaimonic SWB is more about finding one's purpose or meaning in life, growing as a person, and achieving self-actualization (De Vos et al., 2013). Hedonic SWB is commonly subdivided into three parts (Diener, 1984): positive affect (the short-term presence of positive emotions), negative affect (the shortterm absence of negative emotions), and cognitive evaluation (long-term life satisfaction). The distinction between hedonic and eudaimonic SWB is not always clear: Some aspects of (hedonic) cognitive life satisfaction could be related to (eudaimonic) self-actualization. 
Subjective well-being is usually measured by individuals' self-reported answers to survey questions, but this task can be accomplished in different ways. Although assessments of real-time (now) or prospective (in the future) SWB are possible, people are most often asked retrospectively about their recent SWB. (The temporal variability issues associated with these different measurement methods are beyond the scope of this study; see discussions by Abou-Zeid and Ben-Akiva (2012) or Kahneman et al. (1997) for details.) Several well-established psychometric instruments exist for measuring retrospective SWB, most using Likert-type or semantic differential scales (Ettema et al., 2010; De Vos et al., 2013; Mokhtarian, in progress). Measurement scales of the affective components of hedonic SWB include the Positive and Negative Affect Schedule (PANAS), the Swedish Core Affect Scale (SCAS), and the Scale of Positive and Negative Experience (SPANE) (Diener et al., 2010; Västfjäll et al., 2002; Watson et al., 1988). Scales of the cognitive component of hedonic SWB include the Satisfaction with Life Scale (SWLS) and the Personal Well-Being Index (PWI) (Cummins, Eckersley, Pallant, Van Vugt, \& Misajon, 2003; Diener et al., 1985). Eudaimonic SWB is less consistently constructed; scales include the Personal Well-Being Scale (PWS), the Questionnaire for Eudaimonic Well-Being (QEWB), and the Flourishing Scale (FS) (Diener et al., 2010; Ryff, 1989; Waterman et al., 2010).

The existence of easy-to-use questionnaires is one reason why SWB approacheshedonic ones in particular-have begun to be analyzed in transportation studies (Nordbakke \& Schwanen, 2014). Nevertheless, research challenges remain. While some instruments, such as PANAS, are designed to be used over different temporal scales (Watson et al., 1988), many cognitive and eudaimonic questionnaires include items about 
life in general that cannot be easily translated to a shorter time-frame or to a particular domain. Furthermore, most standard SWB metrics have not been comprehensively tested or applied specifically to the transportation area; one exception is described in the following section. More fundamental research is necessary to define reliable and validated measures of SWB that can be used to analyze travel well-being in general, for specific modes, or for individual trips. The research presented in this chapter aims to address this gap.

\subsubsection{The Satisfaction with Travel Scale (STS)}

Ettema and colleagues (Ettema et al., 2011) have developed a measure of hedonic SWB, the Satisfaction with Travel Scale (STS), specific to the travel domain. The first part of the STS derives from the Swedish Core Affect Scale (Västfjäll et al., 2002), which is in turn based on Russell's (1980) circumplex model of core affect. In this framework, emotions or moods can be represented on a two-dimensional surface: one dimension (pleasure or valence) ranges from pleasure to displeasure or positive to negative, and the other dimension (arousal or activation) ranges from activation to deactivation. For example, feeling relaxed or serene would be examples of positive deactivation, while feeling excited or enthusiastic would be positive activation. The second component of the STS measures overall cognitive evaluations about travel.

The STS is usually measured by nine pairs of adjectives or statements on a sevenpoint $(-3$ to +3$)$ semantic differential scale, although early versions used nine-point scales (Ettema et al., 2011; Olsson, Friman, Pareigis, \& Edvardsson, 2012). The STS was designed (Ettema et al., 2011) to measure three aspects of travel SWB or travel satisfaction: 
core affect as ranging from negative activation to positive deactivation (PD), core affect as ranging from negative deactivation to positive activation (PA), and cognitive evaluation (CE), each with three items. (Table 5.2, presented later, lists these items.) The same research team developed another metric, also called the Satisfaction with Travel Scale, in an earlier article (Jakobsson Bergstad et al., 2011), but it includes completely different items and measurement scales. While a few studies use this earlier version of the STS (Cao, 2013; Cao \& Ettema, 2014), most research instead utilizes the Ettema et al. (2011) version or its variants.

The number of studies employing the STS has grown in recent years. Study areas have expanded beyond Sweden to include the Netherlands (Ettema, Gärling, Olsson, Friman, \& Moerdijk, 2013), Belgium (De Vos et al., 2015), China (Ye \& Titheridge, 2017), Canada (Zhao \& Lee, 2013), and the United States (Smith, 2017; Zhao \& Lee, 2013). While most applications aim to measure travelers' SWB while on a recent commute trip to or from work or school, some have applied the STS to other situations: typical commute trips (Zhao \& Lee, 2013), recent leisure trips (De Vos et al., 2015), hypothetical travel agendas (Ettema et al., 2011), and travel in general (Friman et al., 2013). An interesting study (Suzuki et al., 2014) examined STS for trip segments (on a multistage trip) and the entire trip, suggesting that averaging segment-specific assessments with duration weights fits the data better than assuming peak-end weighting (Kahneman, 2000). Most analyses also compare STS across travel modes, but some have focused solely on car (Ettema et al., 2013) or public transit (Olsson et al., 2012; Taniguchi, Grääs, \& Friman, 2014) travelers.

Seven studies, in addition to this one, have examined the measurement structure of the STS in various contexts and with varying conclusions; see Table 5.1. Some of the 
discrepancies among these studies' findings could be the result of applying slightly different measures to varied geographic and transportation contexts or using different analysis techniques. Most researchers have used the original nine-item STS, but Ye and Titheridge (2016) used a seven-item subset, and Smith (2016) had a seven-item variant to reduce respondent burden. Most studies employ confirmatory factor analysis (CFA) - a subset of structural equation modeling (SEM) - to examine whether empirical data back up the hypothesized three-factor structure, while others use exploratory factor analysis (EFA) or put the measurement model within a larger SEM framework. One difference among the findings relates to measurement invariance: Friman et al. (2013) concluded that a three-factor STS structure did not vary across urban areas or travel modes, while De Vos et al. (2015) showed that a two-factor STS was structurally distinct for different travel modes. 
Table 5.1 Studies measuring the Satisfaction with Travel Scale or its variants

\begin{tabular}{|c|c|c|c|c|c|c|c|}
\hline Citation & $N$ & Travel behavior & Study area & Year & Method $^{a}$ & Factors $^{b}$ & Notes \\
\hline Ettema et al., 2011 & 155 & $\begin{array}{l}\text { Hypothetical day travel } \\
\text { agenda }\end{array}$ & $\begin{array}{l}\text { Karlstad } \\
\text { University, } \\
\text { Sweden }\end{array}$ & $\mathrm{n} / \mathrm{a}$ & Average & $\begin{array}{l}3(\mathrm{PD}, \mathrm{PA}, \\
\mathrm{CE}), 1 \text { higher- } \\
\text { order (TS) }\end{array}$ & 9-point scales \\
\hline $\begin{array}{l}\text { Olsson, Friman, } \\
\text { Pareigis, \& } \\
\text { Edvardsson, } 2012\end{array}$ & 189 & Travel by public transit & $\begin{array}{l}\text { Karlstad \& } \\
\text { Gothenburg, } \\
\text { Sweden }\end{array}$ & $\mathrm{n} / \mathrm{a}$ & $\begin{array}{l}\text { EFA (PCA), } \\
\text { CFA }\end{array}$ & $\begin{array}{l}3(\mathrm{PD}, \mathrm{PA}, \\
\mathrm{CE}), 1 \text { higher- } \\
\text { order (TS) }\end{array}$ & 9-point scales \\
\hline $\begin{array}{l}\text { Friman, Fujii, } \\
\text { Ettema, Gärling, \& } \\
\text { Olsson, } 2013\end{array}$ & $\begin{array}{r}951 \\
791\end{array}$ & $\begin{array}{l}\text { Travel in general; } \\
\text { Most recent commute } \\
\text { trip to, from work }\end{array}$ & $\begin{array}{l}\text { Stockholm, } \\
\text { Gothenburg, \& } \\
\text { Malmö, Sweden }\end{array}$ & $\mathrm{n} / \mathrm{a}$ & CFA & $\begin{array}{l}3(\mathrm{PD}, \mathrm{PA}, \\
\mathrm{CE}), 1 \text { higher- } \\
\text { order (TS) }\end{array}$ & $\begin{array}{l}\text { Invariance testing } \\
\text { by urban area, } \\
\text { commute mode }\end{array}$ \\
\hline Smith, 2017 & 828 & $\begin{array}{l}\text { Most recent work } \\
\text { commute trip }\end{array}$ & $\begin{array}{l}\text { Portland, Oregon, } \\
\text { United States }\end{array}$ & 2012 & CFA & $\begin{array}{l}2 \text { (Affective, } \\
\text { Cognitive) }\end{array}$ & $\begin{array}{l}7 \text { items, some } \\
\text { changes }\end{array}$ \\
\hline Zhao \& Lee, 2013 & 1,831 & $\begin{array}{l}\text { Typical, most recent } \\
\text { work/school commute }\end{array}$ & $\begin{array}{l}\text { Canada, United } \\
\text { States }\end{array}$ & 2012 & CFA & $\begin{array}{l}3(\mathrm{PD}, \mathrm{PA}, \\
\mathrm{CE})\end{array}$ & \\
\hline $\begin{array}{l}\text { De Vos, Schwanen, } \\
\text { Van Acker, \& } \\
\text { Witlox, } 2015\end{array}$ & 1,411 & Most recent leisure trip & Ghent, Belgium & 2012 & $\begin{array}{l}\text { EFA (PAF, } \\
\text { promax) }\end{array}$ & $\begin{array}{l}2 \text { (Affective, } \\
\text { Cognitive) }\end{array}$ & $\begin{array}{l}\text { Invariance testing } \\
\text { by transport } \\
\text { mode }\end{array}$ \\
\hline $\begin{array}{l}\text { Ye \& Titheridge, } \\
2016\end{array}$ & 1,215 & $\begin{array}{l}\text { Most recent work } \\
\text { commute trip }\end{array}$ & Xi'an, China & 2013 & SEM & $1(\mathrm{TS})$ & 7 items \\
\hline This study & 656 & $\begin{array}{l}\text { Most recent work } \\
\text { commute trip }\end{array}$ & $\begin{array}{l}\text { Portland, Oregon, } \\
\text { United States }\end{array}$ & 2016 & $\begin{array}{l}\text { EFA (PAF, } \\
\text { oblimin), } \\
\text { CFA }\end{array}$ & $\begin{array}{l}3(\mathrm{PD}, \mathrm{PA}, \\
\mathrm{CE}), 1 \text { higher- } \\
\text { order (TS) }\end{array}$ & $\begin{array}{l}9 \text { items, some } \\
\text { changes }\end{array}$ \\
\hline
\end{tabular}

${ }^{\mathrm{a}} \mathrm{EFA}=$ exploratory factor analysis; $\mathrm{PCA}=$ principal component analysis; $\mathrm{PAF}=$ principal axis factoring; $\mathrm{CFA}=\mathrm{confirmatory}$ factor analysis; SEM = structural equation modeling

${ }^{\mathrm{b}} \mathrm{PD}=$ positive deactivation; $\mathrm{PA}=$ positive activation; $\mathrm{CE}=$ cognitive evaluation; $\mathrm{TS}=$ travel satisfaction. 
Results also differ as to the appropriate number and arrangement of latent constructs measured by the STS. Applying CFA to two different Swedish datasets, Olsson et al. (2012) and Friman et al. (2013) confirmed that their data fit the hypothesized threefactor (PD, PA, CE) model of STS with a second-order factor (travel satisfaction); a singlefactor model did not fit the data. However, both sets of authors allowed for the covariance of errors between some items (as suggested by modification indices); without these error covariances, the three-factor models had unsatisfactory fits (Friman et al., 2013; Olsson et al., 2012). Using data collected from Canadians and Americans through Amazon's Mechanical Turk, Zhao and Lee (2013) confirmed the three-factor STS in the context of a multiple indicators, multiple causes (MIMIC) SEM framework. Ye and Titheridge (2016) examined only a single-factor version of STS, also within a MIMIC framework, and found satisfactory fit. In comparison, De Vos et al. (2015) suggested a two-factor model (affective, cognitive) of STS fit their data better than a three-factor model, as evidenced by principal axis factoring (a type of EFA) and reliability analysis. With fewer items, Smith (2016) also confirmed a two-factor model of STS fit his commuting dataset, but only after adding some error covariances. In both two-factor models, one item had a smaller standardized loading $(<0.50)$ on the affective construct. These results suggest that more research is needed to examine whether a two-factor or a three-factor STS representation of SWB in the travel domain is more appropriate, and if there should be any changes to the items included in the STS. This study attempts to address these tasks. 


\subsubsection{Travel satisfaction}

The cognitive aspects of hedonic SWB have also been investigated in less systematic ways in the travel domain: by asking about overall satisfaction (on Likert-type scales from very dissatisfied to very satisfied) with travel in general or with specific modes or recent trips. While travel satisfaction questions are likely more about cognitive evaluation than positive/negative affect (De Vos et al., 2013), they may be partially measuring some emotional aspects or even values of productive travel time use (Chapter 4). Studies typically find that at least half of the subjects are satisfied with their commutes or a recent trip. Travel satisfaction questions have been asked in the U.S. (Wachs et al., 1993; Archer et al., 2013; Milakis et al., 2015), Canada (St-Louis et al., 2014), Australia (Young \& Morris, 1981), Europe (Rasouli \& Timmermans, 2014a, 2014b; Susilo et al., 2017), China (Mao et al., 2015), and elsewhere (Abou-Zeid \& Ben-Akiva, 2011).

\subsubsection{Travel liking}

An early and relatively common approach to investigating the PUT concept involves asking about a general affinity for travel, known as travel liking (Mokhtarian \& Salomon, 2001; Ory \& Mokhtarian, 2005). These questions likely measure travel affect (discussed in the following section), yet they are common enough to warrant a separate mention. Respondents are typically asked how much they enjoyed or enjoy (on a scale from strongly dislike to strongly like) travel in general, travel by specific modes or for specific purposes, or traveling on a recent trip. Short-distance commuting to work has received the greatest attention (Ory et al., 2004; Turcotte, 2006; Gatersleben \& Uzzell, 2007); based on previous studies, only about $30-40 \%$ of people dislike commuting. Travel liking has been 
investigated in various Western countries, including the U.S. (Curry, 2000; Ory \& Mokhtarian, 2005; Ory et al., 2004), Canada (Turcotte, 2006), the United Kingdom (Gatersleben \& Uzzell, 2007), and France (Mokhtarian, Papon et al., 2015).

\subsubsection{Measuring travel affect}

Some research has measured travelers' feelings, emotions, and moods from the travel experience; i.e., the affective rather than the cognitive aspects of SWB. As with travel satisfaction and liking, travel affect is typically elicited retrospectively, using Likert-type scales and questions about pleasantness, happiness, enjoyment, relaxation, excitement, and more. Other studies use qualitative methods to examine affective motivations for travel behaviors, particularly the use of cars instead of public transit. These affective travel motives revolve around the comfort and privacy of personal space; "time out" (Jain \& Lyons, 2008) to escape obligations, be alone, relax, and do nothing; time to transition between life roles; and pleasures from feeling the wind, smelling the environment, or just moving. The variety of item wordings, the broad scope of affective motivations, and the potential for overlap with cognitive or eudaimonic aspects of SWB all suggest a research need to define more consistent and comprehensive measures of travel affect, which is a goal of this study.

Although research suggests that commuting can be a very stressful activity (Koslowsky, Kluger, \& Reich, 1995), most studies of travel affect find half or more of the subjects are happy when they travel or consider a recent (commute) trip to be pleasant. In fact, affective aspects were rated as being equally important to instrumental factors (flexibility, convenience, and cost) in one study of leisure travel (Anable \& Gatersleben, 
2005). Travel affect has been examined primarily in the United Kingdom (Anable \& Gatersleben, 2005; Gardner \& Abraham, 2007; Gatersleben \& Uzzell, 2007; Mann \& Abraham, 2006; Steg, 2005; Thomas \& Walker, 2015) and continental Europe (Duarte et al., 2010; Mokhtarian, Papon et al., 2015), but also in the U.S. (Archer et al., 2013; Morris \& Guerra, 2015a, 2015b) and Asia (Loo et al., 2015; Rhee et al., 2013). Some questions have even been included in national representative samples like the French National Travel Survey and the American Time Use Survey.

\subsubsection{Measuring travel eudaimonia}

Far fewer studies have investigated the eudaimonic aspects of travel well-being. Existing SWB instruments are not easily applied to the travel domain, and eudaimonic responses to travel or symbolic motivations for traveling may be more implicit and less easily measured in a questionnaire format. Despite these challenges, a growing body of research has investigated the psychosocial benefits of travel and noninstrumental reasons for traveling, often for the purposes of understanding driving and car use behaviors (Gatersleben, 2014; Steg, 2005) or from a sociological perspective (Watts \& Urry, 2008). This literature suggests that symbolic motives for travel (especially by automobile) fit into themes of freedom, independence, and autonomy; power and control; ontological security; variety and a spirit of adventure; self-confidence and competence in a skill; status and prestige; possession and ownership; (lack of) environmental consciousness; and identity and self-expression (Ellaway et al., 2003; Gardner \& Abraham, 2007; Gatersleben, 2014; Gim, 2015; Handy et al., 2005; Hiscock et al., 2002; Jain \& Lyons, 2008; Loo et al., 2015; Mann \& Abraham, 2006; Steg, 2005; Zhao \& Zhao, 2015). 


\subsubsection{Determinants of travel well-being}

Examinations of the transportation-SWB relationship at an individual level have summarized several pathways by which travel can affect well-being (De Vos et al., 2013; Ettema et al., 2010; Mokhtarian, in progress; Nordbakke \& Schwanen, 2014). The most relevant paths for the purposes of this study are those that influence short-term trip-specific SWB through travel experiences during destination-oriented travel, and instances where travel is the activity (e.g., outdoor recreation). Given that transportation affects SWB, it logically follows that expectations or concerns about travel-related well-being may have the potential to affect travel decisions and travel behavior. For instance, travelers may consider expected short-term SWB impacts when choosing travel modes or routes (AbouZeid \& Ben-Akiva, 2014). Scholars have acknowledged this bidirectional relationship (De Vos et al., 2013; Mokhtarian, in progress), but relatively few studies have empirically examined potential determinants of SWB in the travel domain. A summary of findings from those studies follows.

Not surprisingly, several trip and transportation characteristics appear to be associated with travel well-being. Modal effects are prominent; inherent differences among transport modes directly affect travel experiences and how each mode acts as a symbol. Travel SWB — measured in ways including the STS, travel satisfaction, and travel likingis consistently rated more positively for walking and bicycling than for automobile travel, and public transit use is often rated more negatively (Anable \& Gatersleben, 2005; De Vos et al., 2013; De Vos et al., 2015; De Vos et al., 2016; Duarte et al., 2010; Ettema et al., 2011; Friman et al., 2013; Gatersleben \& Uzzell, 2007; LaJeunesse \& Rodríguez, 2012; Mao et al., 2015; Martin, Goryakin, \& Suhrcke, 2014; Morris \& Guerra, 2015a; Olsson et 
al., 2013; Rhee et al., 2013; Smith, 2017; St-Louis et al., 2014; Susilo et al., 2017; Thomas \& Walker, 2015; Turcotte, 2006; Ye \& Titheridge, 2017; Zhao \& Lee, 2013). Some modal differences may result from availability limitations and self-selection: For instance, people who walk and bicycle often may live in places with more options, while some people who drive or use transit may have fewer alternatives. Yet, it is more likely that these differences do result from intrinsic characteristics of the modes themselves: Walking and bicycling are physically active activities that take place outdoors; traveling by transit involves sharing space in close proximity to strangers; and car commuters in major cities often experience congestion.

Travel time and trip purpose may also affect travel well-being. Satisfaction with the travel experience and travel liking tends to decrease with longer trip distances or durations (Milakis et al., 2015; Morris \& Guerra, 2015b; Olsson et al., 2013; Ory \& Mokhtarian, 2005, 2009; Rasouli \& Timmermans, 2014a; Smith, 2017; Stone \& Schneider, 2016; Susilo et al., 2017; Turcotte, 2006; Wachs et al., 1993). However, more-detailed studies indicate that travel time may be nonlinearly associated with travel SWB, increasing to a peak at around 15 minutes before decreasing with a long tail (Milakis et al., 2015; Wachs et al., 1993; Young \& Morris, 1981). Long-distance travel is liked more than short-distance travel (Mokhtarian \& Salomon, 2001), but this may be partially due to confounding travel liking with liking activities at the destination (e.g., recreational travel, tourism, visiting family and friends). Within short-distance travel, work and school commutes seem to be less positive than trips for other purposes (Mokhtarian, Papon et al., 2015; Mokhtarian \& Salomon, 2001; Morris \& Guerra, 2015a; Ory \& Mokhtarian, 2005), perhaps due to anticipation of or preparation for these types of mandatory activities. 
Notably, few objectively measured demographic or socioeconomic traveler characteristics are consistently associated with SWB in the travel domain. An exception is age: Satisfaction with travel, a positive affect about travel, and travel-related SWB (for a particular trip) appear to be higher among older travelers (Archer et al., 2013; Jakobsson Bergstad et al., 2011; Mokhtarian, Papon et al., 2015; Olsson et al., 2013; Ory \& Mokhtarian, 2005; Rasouli \& Timmermans, 2014a, 2014b; St-Louis et al., 2014; Ye \& Titheridge, 2017). This finding may suggest the importance of broader life stage, lifestyle, or cultural influences that transcend more traditional socio-demographic measures. Instead, the attitudes and personalities of travelers seem to be more directly linked to travel wellbeing (De Vos et al., 2016; Ory \& Mokhtarian, 2009; Steg, 2005; St-Louis et al., 2014). For instance, pro-environmental attitudes were associated with travel liking for nonautomobile modes (Ory \& Mokhtarian, 2005).

This review highlights the need for further research to identify additional determinants of travel SWB, particularly with respect to traveler characteristics. There is a need to examine attitudes and nontraditional socio-demographic attributes more closely. Further, most associations have been with the STS, travel liking, or travel satisfaction, leaving eudaimonic SWB aspects nearly untouched. This study attempts to rectify some of these limitations.

\subsubsection{Summary}

To summarize, although many established psychometric instruments exist for measuring hedonic (affective and cognitive aspects) and eudaimonic SWB, few have been adapted for use in studying SWB in the travel domain. One exception is the STS, which 
has been examined in several different contexts and is being used in an increasing number of studies. Most (quantitative) research in this area uses ad-hoc measures of travel satisfaction and travel liking, paying scant attention to travel eudaimonia. The travel behavior field could benefit from more reliable and comprehensive scales for measuring travel well-being, especially with respect to affective and eudaimonic aspects. In addition, the STS requires further validation in a U.S. context and examination of whether a twofactor or a three-factor structure is more appropriate. Finally, additional research is needed to identify factors associated with travel SWB, particularly the identification of associated socio-demographic and attitudinal traveler characteristics. This study attempts to address these research needs by providing stronger measures of travel well-being and further evidence of potential determinants.

\subsection{Data and methods}

The analyses presented in this chapter are part of a broader study investigating the positive utility of travel (PUT) concept and the effects of a PUT on mode choice. This PUT study included a 30-minute online questionnaire survey administered to working and commuting adults in the Portland, Oregon, region. Respondents were asked to report detailed information about their most recent commute trip from home to work, including information on travel affect, travel eudaimonia, and the satisfaction with travel scale. Single-item questions about travel satisfaction and travel liking were also asked, but they are not used for the purposes of this study. Data were collected between mid-October and mid-December 2016, and participants were primarily recruited via email at their place of employment. Although 791 people started the survey, only 682 people completed enough 
questions to be used in these analyses. For more information on the data collection process, see Chapter 3. Descriptive statistics of the sample are shown in Table 4.2.

\subsubsection{Measures of commute well-being}

\subsubsection{Satisfaction with Travel Scale}

The questions and items of the Satisfaction with Travel Scale (STS) were borrowed and adapted from previous studies employing the STS (De Vos et al., 2015; Ettema et al., 2011; Friman et al., 2013; Olsson et al., 2011; Smith, 2017). Nine paired items were measured on seven-point semantic differential scales. For each pair of statements (one to the left, one to the right), respondents were instructed to "select the choice that best corresponds to your overall experience traveling on your most recent commute to work." Following Smith (2016), the original items developed by Ettema et al. (2011) were revised slightly to better fit an American and English-language context, and to better match opposite edges of the two-dimensional core affect concept (Russell, 1980, 2003). In particular, hurried / relaxed and tired / alert had less than perfectly opposing definitions, so these were changed to tense / relaxed and tired / energized. The item fed up / engaged was dropped for the same reason, and worried / confident was clarified to refer to arrival time confidence. Two paired statements were added to Smith's STS following some of the recommendations of De Vos et al. (2015): distressed / content to replace stressed / calm, and sad / happy for a valence-only item (in addition to displeasing / enjoyable). Table 5.2 presents and compares the items included in this version of the STS with those from previous versions. 
Table 5.2 Items included in the Satisfaction with Travel Scale

\begin{tabular}{|c|c|c|c|}
\hline Citation & Positive deactivation & Positive activation & Cognitive evaluation \\
\hline Olsson et al., $2011^{1}$ & $\begin{array}{l}\text { - Very tense / relaxed } \\
\text { - Very stressed / calm } \\
\text { - Very worried / confident }\end{array}$ & $\begin{array}{l}\text { - Very unengaged / engaged } \\
\text { - Very bored / enthusiastic } \\
\text { - Very tired / excited }\end{array}$ & $\begin{array}{l}\text { - Worst / Best trip I can imagine } \\
\text { - Worked very poorly / well } \\
\text { - Very low / high standard }\end{array}$ \\
\hline Ettema et al., $2011^{1}$ & $\begin{array}{l}\text { - Time pressed / Relaxed } \\
\text { - Stressed / Calm } \\
\text { - Worried I would not / } \\
\text { Confident I would be in time }\end{array}$ & $\begin{array}{l}\text { - } \text { Fed up / Engaged } \\
\text { - } \text { Bored / Enthusiastic } \\
\text { - } \text { Tired / Alert }\end{array}$ & $\begin{array}{l}\text { - Travel was worst / best I can think of } \\
\text { - Travel worked well / poorly } \\
\text { - Travel was low / high standard }\end{array}$ \\
\hline Friman et al., $2013^{1}$ & $\begin{array}{l}\text { 1. Very hurried / relaxed } \\
\text { 2. Very stressed / calm } \\
\text { 3. Very worried / confident }\end{array}$ & $\begin{array}{l}\text { 1. Very fed up / engaged } \\
\text { 2. Very bored / enthusiastic } \\
\text { 3. Very tired / alert }\end{array}$ & $\begin{array}{l}\text { 1. Worst / Best imaginable } \\
\text { 2. Worked very poorly / well } \\
\text { 3. Very low / high standard }\end{array}$ \\
\hline This study & $\begin{array}{l}\text { 1. I was very distressed / content } \\
\text { 2. I was very tense / relaxed }\end{array}$ & $\begin{array}{l}\text { 1. I was very sad / happy } \\
\text { 2. I was very tired / energized } \\
\text { 3. I was very bored / enthusiastic }\end{array}$ & $\begin{array}{l}\text { 1. My trip was displeasing / enjoyable } \\
\text { 2. My trip went poorly / smoothly } \\
\text { 3. My trip was the worst / best I can imagine } \\
\text { 4. I was worried I wouldn't / confident I would } \\
\text { arrive on time }\end{array}$ \\
\hline Citation & Affective evaluation & & Cognitive evaluation \\
\hline De Vos et al., 2015 & $\begin{array}{l}\text { 1. Hurried / Relaxed } \\
\text { 2. Stressed / Calm } \\
\text { 3. Worried / Confident }\end{array}$ & $\begin{array}{l}\text { 4. Bored / Enthusiastic } \\
\text { 5. Fed up / Engaged } \\
\text { 6. Tired / Alert }\end{array}$ & $\begin{array}{l}\text { 1. Travel was worst / best I can think of } \\
\text { 2. Travel did not work / worked out well } \\
\text { 3. Travel was low / high standard }\end{array}$ \\
\hline Smith, 2017 & $\begin{array}{l}\text { 1. Not enjoyable / Enjoyable } \\
\text { 2. Tired / Excited } \\
\text { 3. Bored / Enthusiastic }\end{array}$ & $\begin{array}{l}\text { 4. Tense / Relaxed } \\
\text { 5. Worried / Confident that you } \\
\text { would arrive on time }\end{array}$ & $\begin{array}{l}\text { 1. My trip was the worst / best I can imagine } \\
\text { 2. My trip went poorly / smoothly }\end{array}$ \\
\hline
\end{tabular}

${ }^{1}$ The items in these studies were translated into English by the listed authors, which explains some of their differences.

Note: The numbered items are listed in order of the magnitude of their factor analysis loadings on each construct. The bulleted items come

from studies that did not examine the measurement structure of the STS in a similar way. 


\subsubsection{Travel affect}

Measures of travel affect were adapted from existing psychological instruments for affective SWB. A PANAS-type approach was deemed most useful, since it is simple to administer and has been designed to work with shorter time scales (Watson et al., 1988). Question wording followed the PANAS: Respondents were instructed to first think "about yourself and your most recent commute to work," and then "indicate to what extent you felt" each of 20 adjectives while commuting by their chosen transportation mode. Each item was rated on the common PANAS five-point Likert-type scale (see Table 5.3). Due to the length of the survey, the full 20-item PANAS was not used. Instead, the first block of travel affect questions was composed of the 10 items from the international short-form version (I-PANAS-SF), which has been psychometrically validated (Thompson, 2007).

A multistage process was used to round out the second block of 10 travel affect items (see Chapter 3). First, a master list of adjectives or short phrases relating to affect, emotion, or mood were pulled from standard psychological affect scales (PANAS, PANAS-X, I-PANAS-SF, SCAS, and SPANE) and travel behavior literature (Anable \& Gatersleben, 2005; Diana, 2008; Ellaway et al., 2003; Gatersleben \& Uzzell, 2007; Handy et al., 2005; Loo et al., 2015; Milakis et al., 2015; Mokhtarian, Salomon, \& Singer, 2015; Morris \& Guerra, 2015a; Rhee et al., 2013; Steg, 2005; Thomas \& Walker, 2015). Next, about 100 of the most promising of these adjectives were included in a PANAS-type small sample questionnaire. Finally, the remaining 10 items were selected from this list based on three considerations: inclusion in another psychometric instrument (like the PANAS-X), frequent association with travel (from the literature and the small sample survey), and lack 
of overlap with constructs already in the I-PANAS-SF or the STS. Table 5.3 shows the question wording and two blocks of items used to measure travel affect.

\section{Table 5.3 Questions/items used to measure travel affect}

\begin{tabular}{lll}
\hline Question & \multicolumn{2}{l}{ Thinking about yourself and your most } \\
& $\begin{array}{l}\text { recent commute to work, indicate to what } \\
\text { extent you felt the following while }\end{array}$ \\
\multirow{3}{*}{ Scale } & Very slightly or not at all; A little; \\
& Moderately; Quite a bit; Extremely
\end{tabular}

a Modes: walking; bicycling; driving an automobile; riding as a passenger in an automobile; riding public transit.

\subsubsection{Travel eudaimonia}

New questions and items were created to measure the eudaimonic aspects of travel well-being, as the adaptation of existing instruments (e.g., PWS, QEWB, and FS) was deemed infeasible. Instead, a similar multistage process was conducted (see Chapter 3). First, a master list of words or short phrases was pulled from existing psychological scales (FS) and travel behavior literature (Anable \& Gatersleben, 2005; Diana, 2008; Ellaway et 
al., 2003; Handy et al., 2005; Loo et al., 2015; Mokhtarian, Salomon, \& Singer, 2015; Ory \& Mokhtarian, 2005; Rhee et al., 2013; Salomon \& Mokhtarian, 1998; Steg, 2005). Next, about 70 of these words were included in the same small-sample questionnaire. Finally, 22 words/phrases were selected based on frequent associations with travel (from the literature and the survey) and coverage of a number of concepts identified in the literature review above (freedom, exploration, control, protection, skill, change, social, nature, identity, and health). Each of these items were grouped into one of three question blocks, reflecting potential motivations for travel: to "fulfill your desire for," "express," or "improve" something (see Table 5.4). A small pilot survey suggested answering these questions on a similar five-point Likert-type scale was difficult, so the response scale was changed to a yes/no checkbox. Table 5.4 shows the question wording and three blocks of items used to measure travel eudaimonia. 


\section{Table 5.4 Questions/items used to measure travel eudaimonia}

\begin{tabular}{|c|c|c|c|}
\hline Question & \multicolumn{3}{|c|}{$\begin{array}{l}\text { Thinking about your most recent commute to work, did __ a allow you, } \\
\text { at least a little, to... }\end{array}$} \\
\hline Scale & \multicolumn{3}{|l|}{ Checkbox } \\
\hline & ...fulfill your desire for: & ...express your: & ...improve your: \\
\hline \multirow[t]{2}{*}{ Items } & Block 1 & Block 2 & Block 3 \\
\hline & $\begin{array}{l}\text { - Variety } \\
\text { - Control } \\
\text { - Adventure } \\
\text { - Companionship } \\
\text { - Freedom } \\
\text { - Privacy } \\
\text { - Safety } \\
\text { - Comfort } \\
\text { - Stress relief } \\
\text { - A routine } \\
\text { - A challenge } \\
\text { - A buffer between home } \\
\text { - } \text { and work } \\
\text { - } \text { or class } \\
\text { - None of the above }\end{array}$ & $\begin{array}{l}\text { - Independence } \\
\text { - Social status } \\
\text { - Self-identity } \\
\text { - Courage } \\
\text { - Mastery of a skill } \\
\text { - Environmental } \\
\text { - } \text { values } \\
\text { - None of the above }\end{array}$ & $\begin{array}{l}\text { - Self-confidence } \\
\text { - Mental health } \\
\text { - Physical health } \\
\text { - None of the above }\end{array}$ \\
\hline
\end{tabular}

${ }^{a}$ Modes: walking; bicycling; driving an automobile; riding as a passenger in an automobile; riding public transit.

\subsubsection{Analysis methods}

The analysis approach for each concept (the STS, travel affect, and travel eudaimonia) followed a roughly similar process. First, exploratory factor analysis (EFA) was used to suggest a likely factor structure. EFA is a common first step towards developing a validated scale, and it has been used to create and validate the STS. Specifically, Horn's parallel analysis was conducted to determine an approximate number of factors, and the EFA applied principal axis factoring with oblique oblimin rotation to 
extract factors. The EFAs were conducted using the paran (Dinno, 2012) and psych (Revelle, 2016) packages in R.

Second, confirmatory factor analysis (CFA) was used to examine hypothesized factor structures. Several specifications were tested based on the EFA results and suggestions from the literature, and a final measurement model was estimated. Four measures of goodness-of-fit were considered: CFI, TLI, RMSEA, and SRMR. Based on suggestions in the literature (Hu \& Bentler, 1999; Kline, 2016; MacCallum, Browne, \& Sugawara, 1996), adequate fits were above 0.90 and good fits were above 0.95 for both CFI and TLI. For RMSEA, adequate fits were below 0.08 and good fits were below 0.05; for SRMR, these cutoffs were 0.10 (adequate) and 0.08 (good). Other CFA considerations included: having moderate standardized loadings $(\geq 0.40)$, identification (at least two indicators per latent variable), a simple structure (no cross-loaded items or item error covariances), and unique constructs (not highly correlated with one another). The CFAs were conducted using the lavaan package (Rosseel, 2012) in R.

Third, potential determinants of travel well-being were examined by estimating a basic structural equation model (SEM) in which exogenous variables predicted each CFA's latent variables, also known as a multiple indicators, multiple causes (MIMIC) model. The MIMIC models were also estimated using the lavaan package (Rosseel, 2012) in R. For the purposes of this study, the exogenous variables included trip characteristics (commute mode, travel time, number of co-travelers), weather (temperature, precipitation), traveler demographics and socioeconomics (individual, household, transportation, and job attributes), and traveler perceptions (satisfaction with typical commute travel time, selfreported ideal commute travel time, teleportation test). A self-reported measure of travel 
usefulness was also included to control for some potential overlap between measures of the values of two aspects of the positive utility of travel: travel-based multitasking (Chapter 4) and positive travel experiences (this chapter). Before the independent variables entered the model, they were examined for multicollinearity issues; variables that were moderately-tostrongly correlated (> 0.40) were removed. See Table 4.2 for a full list of the independent variables and their descriptive statistics.

\subsection{Results}

Analysis results are presented in the following sections. The first section considers the measurement of commute well-being concepts, while the second section investigates their potential determinants. Each section is subdivided by the aspect of SWB investigated: the Satisfaction with Travel Scale, travel affect, and travel eudaimonia.

\subsubsection{Measuring commute well-being}

Table 5.5 summarizes the goodness-of-fit statistics for each of the CFA models described in the following sections. 
Table 5.5 Goodness-of-fit statistics for CFAs of commute well-being

\begin{tabular}{lrrr}
\hline & \multicolumn{3}{c}{ Confirmatory factor analysis } \\
\cline { 2 - 4 } Goodness-of-fit statistics & The STS & Travel affect & Travel eudaimonia \\
\hline$N$ & 656 & 682 & 680 \\
$\chi^{2}(d f)$ & $98.41(24)$ & $369.65(129)$ & $200.77(71)$ \\
$p$-value $\left(\chi^{2}\right)$ & 0.000 & 0.000 & 0.000 \\
CFI $^{\mathrm{a}}$ & 0.967 & 0.923 & 0.971 \\
TLI $^{\mathrm{a}}$ & 0.951 & 0.909 & 0.963 \\
RMSEA $^{\mathrm{b}}$ & 0.080 & 0.063 & 0.052 \\
SRMR $^{\mathrm{c}}$ & 0.031 & 0.060 & 0.072 \\
\hline
\end{tabular}

${ }^{a}$ Good fit is $>0.95$; adequate fit is $>0.90$.

${ }^{\mathrm{b}}$ Good fit is $<0.05$; adequate fit is $<0.08$.

${ }^{\mathrm{c}}$ Good fit is < 0.08; adequate fit is $<0.10$.

\subsubsection{Satisfaction with Travel Scale}

The nine items in the STS were relatively correlated with one another, with item correlations ranging from 0.32 to 0.70 ; see Table 5.6. The three factors extracted from an EFA on STS items together explained about $61 \%$ of the observed variance; full results are shown in Table 5.7. 
Table 5.6 Correlations between items on the STS

\begin{tabular}{|c|c|c|c|c|c|c|c|c|}
\hline \multirow[b]{2}{*}{ \# Item } & \multicolumn{8}{|c|}{ Correlations } \\
\hline & 1 & 2 & 3 & 4 & 5 & 6 & 7 & 8 \\
\hline 1 Tense ... Relaxed & & & & & & & & \\
\hline 2 Bored ... Enthusiastic & 0.37 & & & & & & & \\
\hline 3 Sad ... Happy & 0.43 & 0.52 & & & & & & \\
\hline 4 Tired ... Energized & 0.43 & 0.53 & 0.61 & & & & & \\
\hline 5 Distressed ... Content & 0.61 & 0.47 & 0.67 & 0.53 & & & & \\
\hline 6 Trip went Poorly ... Smoothly & 0.54 & 0.32 & 0.45 & 0.38 & 0.62 & & & \\
\hline 7 Trip was Displeasing ... Enjoyable & 0.58 & 0.55 & 0.58 & 0.53 & 0.69 & 0.70 & & \\
\hline 8 Worried ... Confident arrive on time & 0.44 & 0.36 & 0.36 & 0.36 & 0.46 & 0.55 & 0.54 & \\
\hline 9 Trip was Worst ... Best imagined & 0.41 & 0.42 & 0.50 & 0.41 & 0.54 & 0.59 & 0.63 & 0.47 \\
\hline
\end{tabular}

$\vec{\infty}$ 


\section{Table 5.7 EFA of items on the STS}

\begin{tabular}{lrrr}
\hline & \multicolumn{4}{r}{$\begin{array}{r}\text { Factors and } \\
\text { factor loadings }\end{array}$} \\
\cline { 2 - 5 } Item & 0.38 & 0.35 & 3 \\
\hline Tense ... Relaxed & 0.24 & & 0.54 \\
Bored ... Enthusiastic & & 0.71 & 0.24 \\
Sad ... Happy & & 0.47 & 0.37 \\
Tired ... Energized & & 0.86 & \\
Distressed ... Content & 0.87 & & \\
Trip went Poorly ... Smoothly & 0.71 & & \\
Trip was Displeasing ... Enjoyable & 0.70 & & \\
Worried ... Confident arrive on time & 0.62 & & \\
Trip was Worst ... Best imagined & 0.30 & 0.22 & 0.09 \\
Proportion of variance explained & & & \\
Correlations among factors & & & \\
$\quad$ Factor 2 & 0.77 & & \\
$\quad$ Factor 3 & 0.26 & 0.44 & \\
\hline
\end{tabular}

${ }^{\mathrm{a}}$ Factor loadings $< \pm 0.20$ are not shown.

The literature review suggests that the STS represents three closely related constructs: "positive deactivation" (PD), "positive activation" (PA), and "cognitive evaluation" (CE). Accordingly, and based on the EFA results, a three-factor CFA model of the STS was estimated. The CFA used maximum likelihood estimation with robust standard errors and a Satorra-Bentler scaled test statistic (the MLM option in lavaan) (Satorra \& Bentler, 1994); 20 cases with partially missing data were excluded, for a sample size of 656. A three-factor model provided a relatively good fit to the data across several statistics $(\mathrm{CFI}=0.967, \mathrm{TLI}=0.951, \mathrm{RMSEA}=0.080, \mathrm{SRMR}=0.031)$, although the RMSEA value indicated barely adequate fit. The three factors matched the PD/PA/CE distinction well: "Positive deactivation" was represented by Distressed ... Content and Tense ... Relaxed; both positive ends are less active. "Positive activation" was represented 
by Sad ... Happy, Tired ... Energized, and Bored ... Enthusiastic; all positive ends are more active. The items that loaded on "Cognitive evaluation"-Trip was Displeasing ... Enjoyable, Trip went Poorly ... Smoothly, Trip was Worst ... Best imagined, and Worried ... Confident arrive on time - are all less about emotions (perhaps with the exception of Displeasing ... Enjoyable) and more about an overall assessment of the trip. All standardized loadings were large but not too large $(0.60<\lambda<0.90)$, and all three constructs had adequate internal reliability (Cronbach's $\alpha>0.70$ ). Results of the CFA are presented in Figure 5.1.

Correlations between the latent variable disturbances were somewhat high (0.790.89), suggesting potential overlapping constructs, therefore a second-order construct ("Commute satisfaction") was included. The model obtained large standardized loadings with a strong contribution from the PA latent variable, suggesting the STS could be a higher-order construct with three lower-level constructs. However, this model could not be statistically distinguished from a three-factor-with-covariances model because it had exactly the same fit (as was expected with identical degrees of freedom, exchanging three covariances for three loadings). 


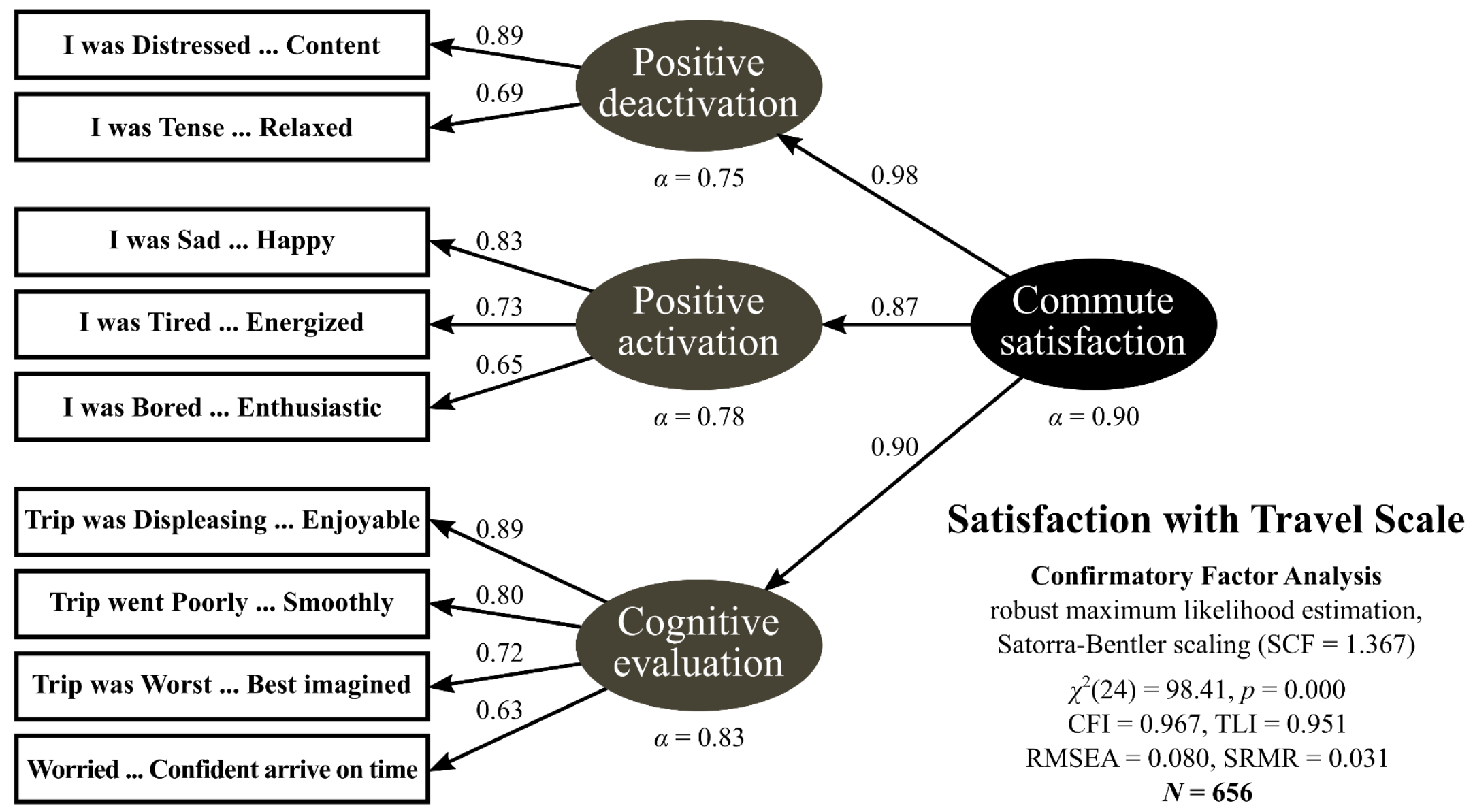

Figure 5.1 CFA of the STS

$\infty$ 
Given that some recent studies rejected the three-factor STS in favor of a two-factor structure (affective, cognitive) (De Vos et al., 2015; Smith, 2017), a two-factor CFA was also estimated (not shown), collapsing the PD/PA constructs. This model had acceptable standardized loadings on the collapsed "Affect" construct (ranging from 0.86 for Distressed ... Content to 0.61 for Bored ... Enthusiastic). However, it also had poorer goodness-of-fit statistics $(\mathrm{CFI}=0.946, \mathrm{TLI}=0.925, \mathrm{RMSEA}=0.099, \mathrm{SRMR}=0.040)$, with an inadequate RMSEA value that had a $90 \%$ confidence interval worse than adequate $(\mathrm{CI}=0.084$ to 0.115$)$.

\subsubsection{Travel affect}

The EFA on the 20 items measuring travel affect suggested four factors that cumulatively explained about $49 \%$ of the observed variance. Item correlations are shown in Table 5.8, and EFA results are presented in Table 5.9. Two of these factors represent negative items, and two represent positive items, as expected. However, two items did not load strongly onto any factor. Ashamed had a low loading on factor 2 (0.22); it also had the fewest responses greater than the first category (4\%). Calm loaded weakly (and negatively) on factor $2(-0.36)$; when reverse coded in the CFA, it had an unacceptably low loading (0.37). For these reasons, both Ashamed and Calm items were removed from further analyses of travel affect. 
Table 5.8 Correlations between items measuring travel affect

\begin{tabular}{|c|c|c|c|c|c|c|c|c|c|c|c|c|c|c|c|c|c|c|c|}
\hline \multirow[b]{2}{*}{ \# Item } & \multicolumn{19}{|c|}{ Correlations } \\
\hline & 1 & 2 & 3 & 4 & 5 & 6 & 7 & 8 & 9 & 10 & 11 & 12 & 13 & 14 & 15 & 16 & 17 & 18 & 19 \\
\hline \multicolumn{20}{|l|}{1 Upset } \\
\hline 2 Hostile & 0.57 & & & & & & & & & & & & & & & & & & \\
\hline 3 Alert & 0.18 & 0.13 & & & & & & & & & & & & & & & & & \\
\hline 4 Ashamed & 0.21 & 0.18 & 0.03 & & & & & & & & & & & & & & & & \\
\hline 5 Inspired & -0.11 & -0.09 & 0.20 & 0.00 & & & & & & & & & & & & & & & \\
\hline 6 Nervous & 0.35 & 0.22 & 0.18 & 0.21 & 0.04 & & & & & & & & & & & & & & \\
\hline 7 Determined & 0.10 & 0.10 & 0.26 & 0.06 & 0.41 & 0.09 & & & & & & & & & & & & & \\
\hline 8 Attentive & 0.08 & 0.08 & $0.65-$ & -0.05 & 0.20 & 0.10 & 0.30 & & & & & & & & & & & & \\
\hline 9 Afraid & 0.28 & 0.21 & 0.15 & 0.13 & 0.03 & 0.62 & 0.13 & 0.11 & & & & & & & & & & & \\
\hline 10 Active & -0.05 & -0.03 & $0.31-$ & -0.06 & 0.52 & 0.09 & 0.38 & 0.35 & 0.10 & & & & & & & & & & \\
\hline 11 Excited & 0.01 & -0.00 & 0.19 & 0.06 & 0.56 & 0.14 & 0.36 & 0.22 & 0.17 & 0.46 & & & & & & & & & \\
\hline 12 Strong & -0.05 & -0.03 & 0.26 & 0.01 & 0.51 & 0.09 & 0.38 & 0.30 & 0.14 & 0.58 & 0.58 & & & & & & & & \\
\hline 13 Vulnerable & 0.23 & 0.16 & 0.25 & 0.02 & 0.15 & 0.43 & 0.23 & 0.25 & 0.48 & 0.31 & 0.23 & 0.27 & & & & & & & \\
\hline 14 Proud & -0.04 & -0.03 & $0.16-$ & -0.03 & 0.50 & 0.06 & 0.32 & 0.21 & 0.10 & 0.43 & 0.56 & 0.62 & 0.19 & & & & & & \\
\hline 15 Angry & 0.57 & 0.59 & 0.16 & 0.14 & -0.01 & 0.25 & 0.16 & 0.09 & 0.25 & 0.05 & 0.09 & 0.03 & 0.22 & 0.02 & & & & & \\
\hline 16 Bold & 0.05 & 0.08 & 0.19 & 0.03 & 0.38 & 0.08 & 0.37 & 0.18 & 0.13 & 0.34 & 0.40 & 0.43 & 0.22 & 0.48 & 0.14 & & & & \\
\hline 17 Frustrated & 0.61 & 0.47 & 0.17 & 0.20 & -0.12 & 0.27 & 0.12 & 0.11 & 0.29 & $-0.10-$ & -0.05 & -0.12 & $0.31-$ & -0.09 & 0.55 & 0.03 & & & \\
\hline 18 Timid & 0.13 & 0.07 & 0.08 & 0.08 & 0.05 & 0.40 & 0.08 & 0.07 & 0.45 & 0.08 & 0.05 & 0.07 & 0.31 & 0.09 & 0.16 & 0.19 & 0.12 & & \\
\hline 19 Calm & -0.26 & -0.23 & $0.09-$ & -0.08 & 0.17 & -0.13 & 0.08 & 0.21 & -0.05 & 0.20 & 0.16 & 0.28 & -0.08 & 0.24 & -0.20 & $0.11-$ & $-0.30-$ & -0.02 & \\
\hline 20 Stressed & 0.55 & 0.40 & 0.14 & 0.18 & -0.11 & 0.44 & 0.09 & 0.07 & 0.33 & $-0.09-$ & $-0.06-$ & -0.13 & $0.27-$ & -0.13 & 0.44 & -0.02 & 0.58 & $0.18-$ & 0.35 \\
\hline
\end{tabular}


Table 5.9 EFA of items measuring travel affect

\begin{tabular}{|c|c|c|c|c|}
\hline \multirow[b]{2}{*}{ Item } & \multicolumn{4}{|c|}{ Factors and factor loadings ${ }^{a}$} \\
\hline & 1 & 2 & 3 & 4 \\
\hline Upset & & 0.78 & & \\
\hline Hostile & & 0.74 & & \\
\hline Alert & & & & 0.68 \\
\hline Ashamed & & 0.22 & & \\
\hline Inspired & 0.72 & & & \\
\hline Nervous & & & 0.75 & \\
\hline Determined & 0.47 & & & \\
\hline Attentive & & & & 0.91 \\
\hline Afraid & & & 0.80 & \\
\hline Active & 0.58 & & & 0.21 \\
\hline Excited & 0.75 & & & \\
\hline Strong & 0.76 & & & \\
\hline Vulnerable & & & 0.51 & \\
\hline Proud & 0.76 & & & \\
\hline Angry & & 0.76 & & \\
\hline Bold & 0.59 & & & \\
\hline Frustrated & & 0.72 & & \\
\hline Timid & & & 0.57 & \\
\hline Calm & 0.20 & -0.36 & & \\
\hline Stressed & & 0.55 & 0.25 & \\
\hline Proportion of variance explained & 0.17 & 0.14 & 0.10 & 0.08 \\
\hline Correlations among factors & & & & \\
\hline Factor 2 & -0.07 & & & \\
\hline Factor 3 & 0.16 & 0.43 & & \\
\hline Factor 4 & 0.37 & 0.14 & 0.19 & \\
\hline
\end{tabular}

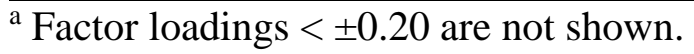

Based on the EFA results, a four-factor CFA model using the 18 remaining travel affect items was estimated. Again, robust maximum likelihood estimation with SatorraBentler scaling was applied, using a sample size of 682. Model goodness-of-fit statistics were within acceptable but not good ranges $(\mathrm{CFI}=0.923, \mathrm{TLI}=0.909, \mathrm{RMSEA}=0.063$ $(\mathrm{CI}=0.056$ to 0.071$)$, SRMR $=0.060)$. Most standardized loadings were acceptable; only a few were less than 0.60 . The CFA model confirmed a four-factor structure of positive 
and negative travel affect, with two modestly correlated (0.49) positive constructs and two modestly correlated $(0.42)$ negative constructs. All constructs exhibited adequate internal reliability (Cronbach's $\alpha>0.70$ ). One negative factor was about "Distress," including feelings of frustration, anger, and stress. Another was related to "Fear," and was measured by Afraid, Nervous, Vulnerable, and Timid. On the positive side, Alert and Attentive loaded on a two-item factor that could be called "Attentiveness." The remaining positive items loaded on an overall "Enjoyment" factor, although there may be fewer similarities among these items than among the items that loaded on other factors. Several attempts to use fewer factors or different arrangements of item loadings yielded inadequate or poorer-fitting models. Results for travel affect CFA are presented in Figure 5.2. 


\section{Travel affect}

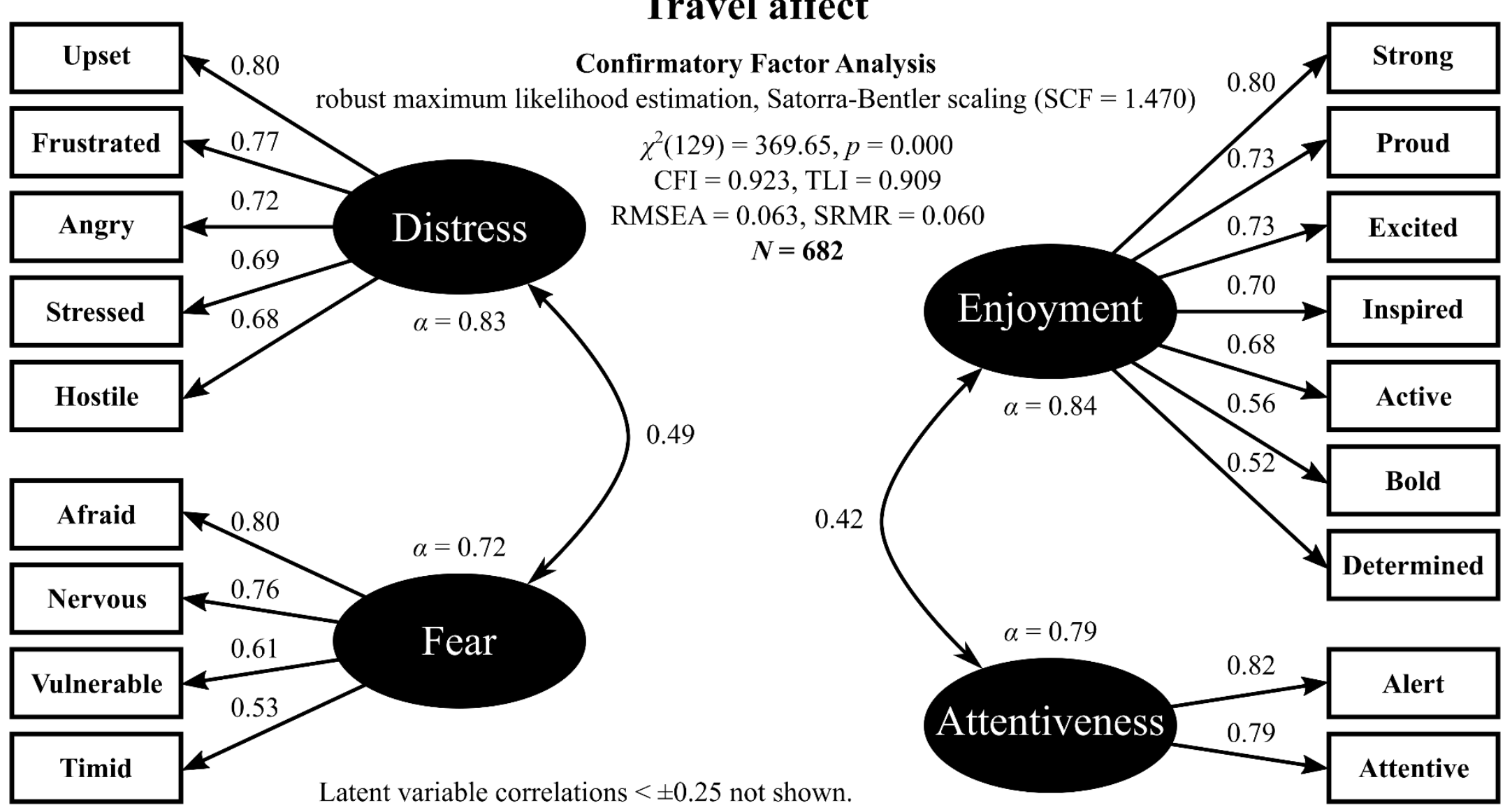

Figure 5.2 CFA of a measurement model of travel affect 


\subsubsection{Travel eudaimonia}

An EFA on 22 items measuring travel eudaimonia extracted four factors that explained about $36 \%$ of observed variance. Results of the EFA are shown in Table 5.11, and item correlations are in Table 5.10. Several items were potentially problematic, based on their EFA loadings. Two items (Companionship and Membership in a group or class) did not load on any factor $(\lambda<0.20)$ and so were removed; these two were also among the items least frequently selected by respondents (9.1\% and $2.5 \%$, respectively). Five other items-Variety, Adventure, A routine, Social status, and Self-identity-had low to moderately low loadings $(\lambda<0.40)$ on multiple factors; retaining these cross-loaded items could have resulted in discriminant validity issues. Despite loading only moderately on two factors in the EFA, the Comfort item was retained and grouped with Safety; an EFA performed after removing the earlier items (not shown) suggested these items were a separate factor. Instead, the Privacy item was removed over discriminant validity concerns despite what appeared to be a good structure; it cross-loaded in the revised EFA, and when included in the CFA it actually fit better (a higher standardized loading and better overall model fit) when grouped with Comfort and Safety than with Freedom, Independence, and Control. In the end, only 14 items measuring travel eudaimonia were retained. 
Table 5.10 Correlations between items measuring travel eudaimonia

\begin{tabular}{|c|c|c|c|c|c|c|c|c|c|c|c|c|c|c|c|c|c|c|c|c|c|}
\hline \multirow[b]{2}{*}{ \# Item } & \multicolumn{21}{|c|}{ Correlations } \\
\hline & 1 & 2 & 3 & 4 & 5 & 6 & 7 & 8 & 9 & 10 & 11 & 12 & 13 & 14 & 15 & 16 & 17 & 18 & 19 & 20 & 21 \\
\hline 1 Variety & & & & & & & & & & & & & & & & & & & & & \\
\hline 2 Control & 0.26 & & & & & & & & & & & & & & & & & & & & \\
\hline 3 Adventure & 0.42 & 0.19 & & & & & & & & & & & & & & & & & & & \\
\hline 4 Companionship & 0.11 & 0.01 & 0.07 & & & & & & & & & & & & & & & & & & \\
\hline 5 Freedom & 0.28 & 0.50 & 0.30 & 0.07 & & & & & & & & & & & & & & & & & \\
\hline 6 Privacy & 0.10 & 0.36 & -0.01 & -0.03 & 0.32 & & & & & & & & & & & & & & & & \\
\hline 7 Safety & 0.18 & 0.21 & 0.04 & 0.14 & 0.28 & 0.24 & & & & & & & & & & & & & & & \\
\hline 8 Comfort & 0.18 & 0.31 & 0.02 & 0.16 & 0.32 & 0.30 & 0.44 & & & & & & & & & & & & & & \\
\hline 9 Stress relief & 0.33 & 0.18 & 0.36 & 0.10 & 0.25 & -0.02 & 0.07 & 0.13 & & & & & & & & & & & & & \\
\hline 10 A routine & 0.11 & 0.17 & 0.15 & 0.06 & 0.25 & 0.04 & 0.19 & 0.21 & 0.17 & & & & & & & & & & & & \\
\hline 11 A challenge & 0.29 & 0.24 & 0.43 & 0.04 & 0.24 & 0.05 & 0.05 & 0.05 & 0.28 & 0.11 & & & & & & & & & & & \\
\hline 12 A buffer & 0.24 & 0.16 & 0.20 & 0.03 & 0.25 & 0.07 & 0.15 & 0.13 & 0.35 & 0.25 & 0.16 & & & & & & & & & & \\
\hline 13 Membership & 0.07 & 0.06 & 0.11 & 0.05 & $0.07-$ & -0.03 & 0.04 & 0.02 & 0.09 & 0.11 & 0.05 & 0.07 & & & & & & & & & \\
\hline 14 Independence & 0.31 & 0.43 & 0.27 & 0.06 & 0.48 & 0.24 & 0.22 & 0.29 & 0.26 & 0.23 & 0.20 & 0.20 & 0.06 & & & & & & & & \\
\hline 15 Social status & 0.11 & 0.15 & 0.11 & 0.02 & 0.17 & 0.21 & 0.14 & 0.09 & 0.15 & 0.10 & 0.06 & 0.16 & 0.14 & 0.22 & & & & & & & \\
\hline 16 Self-identity & 0.25 & 0.19 & 0.32 & 0.08 & 0.31 & 0.05 & 0.17 & 0.13 & 0.34 & 0.23 & 0.24 & 0.31 & 0.13 & 0.31 & 0.34 & & & & & & \\
\hline 17 Courage & 0.15 & 0.12 & 0.37 & 0.06 & 0.20 & 0.01 & 0.08 & 0.06 & 0.23 & 0.12 & 0.44 & 0.15 & 0.11 & 0.16 & 0.22 & 0.32 & & & & & \\
\hline 18 Mastery of a skill & 0.25 & 0.23 & 0.35 & 0.02 & 0.24 & 0.10 & 0.10 & 0.12 & 0.24 & 0.16 & 0.39 & 0.15 & 0.15 & 0.31 & 0.17 & 0.31 & 0.38 & & & & \\
\hline 19 Environmental & 0.25 & -0.00 & 0.30 & 0.06 & $0.18-$ & -0.12 & 0.11 & 0.05 & 0.41 & 0.16 & 0.22 & 0.27 & 0.06 & 0.16 & 0.15 & 0.38 & 0.28 & 0.12 & & & \\
\hline 20 Self-confidence & 0.23 & 0.13 & 0.38 & 0.08 & 0.23 & 0.05 & 0.13 & 0.09 & 0.26 & 0.17 & 0.34 & 0.16 & 0.11 & 0.32 & 0.18 & 0.37 & 0.40 & 0.40 & 0.27 & & \\
\hline 21 Mental health & 0.30 & 0.20 & 0.39 & 0.15 & 0.33 & 0.06 & 0.11 & 0.15 & 0.52 & 0.19 & 0.30 & 0.36 & 0.04 & 0.32 & 0.14 & 0.40 & 0.30 & 0.24 & 0.43 & 0.35 & \\
\hline 22 Physical health & 0.35 & 0.15 & 0.43 & 0.05 & 0.27 & -0.12 & $-0.03-$ & -0.01 & 0.51 & 0.16 & 0.35 & 0.32 & 0.11 & 0.24 & 0.07 & 0.39 & 0.30 & 0.22 & 0.53 & 0.30 & 0.53 \\
\hline
\end{tabular}


Table 5.11 EFA of items measuring travel eudaimonia

\begin{tabular}{|c|c|c|c|c|}
\hline \multirow[b]{2}{*}{ Item } & \multicolumn{4}{|c|}{ Factors and factor loadings ${ }^{a}$} \\
\hline & 1 & 2 & 3 & 4 \\
\hline Variety & 0.33 & & 0.28 & \\
\hline Control & & & 0.72 & \\
\hline Adventure & 0.29 & 0.40 & & -0.20 \\
\hline Companionship & & & & \\
\hline Freedom & & & 0.60 & \\
\hline Privacy & -0.24 & & 0.51 & \\
\hline Safety & & & 0.25 & 0.47 \\
\hline Comfort & & & 0.41 & 0.40 \\
\hline Stress relief & 0.65 & & & \\
\hline A routine & & & & 0.23 \\
\hline A challenge & & 0.52 & & -0.22 \\
\hline A buffer between home and work & 0.44 & & & \\
\hline Membership in a group or class & & & & \\
\hline Independence & & & 0.48 & \\
\hline Social status & & 0.27 & & 0.28 \\
\hline Self-identity & 0.32 & 0.33 & & 0.28 \\
\hline Courage & & 0.68 & & \\
\hline Mastery of a skill & & 0.64 & & \\
\hline Environmental values & 0.64 & & & \\
\hline Self-confidence & & 0.60 & & \\
\hline Mental health & 0.63 & & & \\
\hline Physical health & 0.79 & & & \\
\hline Proportion of variance explained & 0.13 & 0.10 & 0.09 & 0.04 \\
\hline Correlations among factors & & & & \\
\hline Factor 2 & 0.56 & & & \\
\hline Factor 3 & 0.22 & 0.33 & & \\
\hline Factor 4 & 0.05 & 0.05 & 0.24 & \\
\hline
\end{tabular}

${ }^{\mathrm{a}}$ Factor loadings $< \pm 0.20$ are not shown.

These remaining items were considered in a model measuring four constructs of travel eudaimonia. Because the response scale for these items was binary, the CFA employed diagonally weighted least squares estimation with robust standard errors and a mean-and-variance adjusted test statistic (the WLSMV option in lavaan) (Muthén, du Toit, \& Spisic, 1997). No missing-data method was necessary, and 680 full cases were used. 
Overall, the five-factor CFA exhibited good fit statistics $(\mathrm{CFI}=0.971$, TLI $=0.963$, RMSEA $=0.052$, SRMR $=0.072)$. The $90 \%$ confidence interval of the RMSEA value $(C I$ $=0.044$ to 0.060$)$ straddled the line between good and adequate fit. All standardized loadings were within a reasonable range $(0.60<\lambda<0.90)$, and all constructs had sufficient internal reliability (Cronbach's $\alpha>0.70$ ) except for the two-item factor. The four constructs identified were distinct and reasonable. Two items were associated with "Security": Comfort and Safety. This factor was related to the concept of "Autonomy," indicated by Freedom, Independence, and Control. Next, the items Courage, A challenge, Selfconfidence, and Mastery of a skill were all measures of "Confidence." The final construct was about "Health": not just Physical health but also Mental health, Stress relief, A buffer between home and work, and Environmental values. Results from the CFA are shown in Figure 5.3.

Despite the good overall fit of the four-factor measurement model, it did have a few more challenges than the other two ways of measuring commute well-being. Many items were removed because either they did not load on any factor or they loaded on too many factors. Another limitation is that the "Security" factor was measured by only two items. A further challenge is the relatively high correlated disturbances (>0.70) among several pairs of latent variables. These results highlight potential discriminant validity problems with slightly overlapping constructs of travel eudaimonia. Nevertheless, the four factors do mirror findings from the literature about eudaimonic concepts that have been associated with travel behavior. 


\section{Travel eudaimonia}

Confirmatory Factor Analysis

robust diagonally-weighted least-squares estimation, mean-variance adjustment $(\mathrm{SCF}=0.765)$, probit links

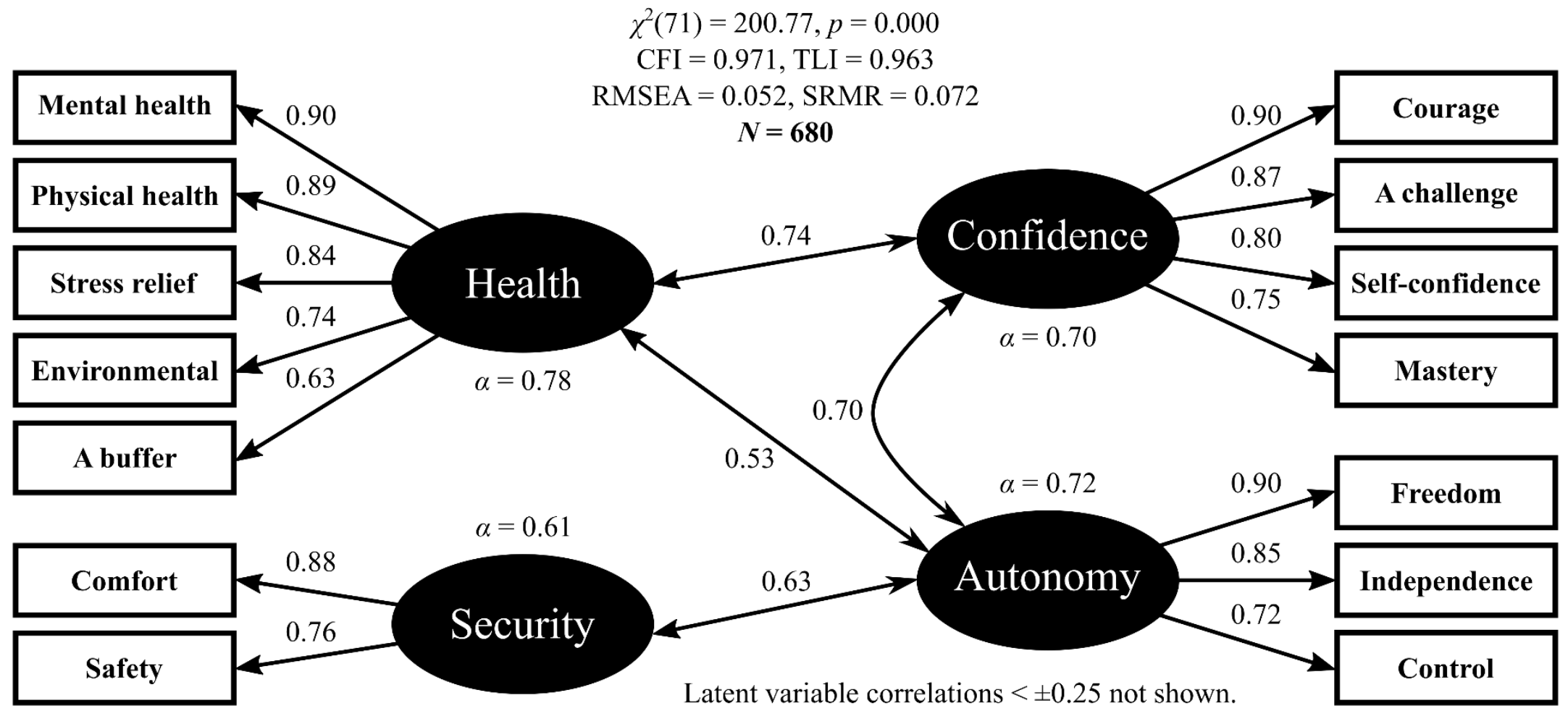

Figure 5.3 CFA of a measurement model of travel eudaimonia 


\subsubsection{Determinants of commute well-being}

\subsubsection{Satisfaction with Travel Scale}

Figure 5.4 presents the distributions of the three individual (and one overall) standardized factor scores for the STS, calculated from the CFA of Figure 5.1 and summarized using box-and-whisker plots for each commute mode. The box plots for each

group show a thick horizontal line at the median, the interquartile range (25th to 75 th percentiles) within the box, and whiskers extending to 1.5 times the interquartile range; outliers are represented by dots located beyond the whiskers. There appear to have been significant modal differences on the factor scores, but these differences were roughly stable across STS constructs, as would be expected by the highly correlated latent variables. Overall, walking and bicycling commuters reported higher-than-average travel satisfaction ratings, especially on the items making up "Cognitive evaluation." Transit riders and auto passengers had roughly average ratings; auto drivers had the lowest STS scores, on average. Despite these modal trends, there was large variation in STS even within modes, suggesting a role for many other explanatory factors beyond mode choice. 

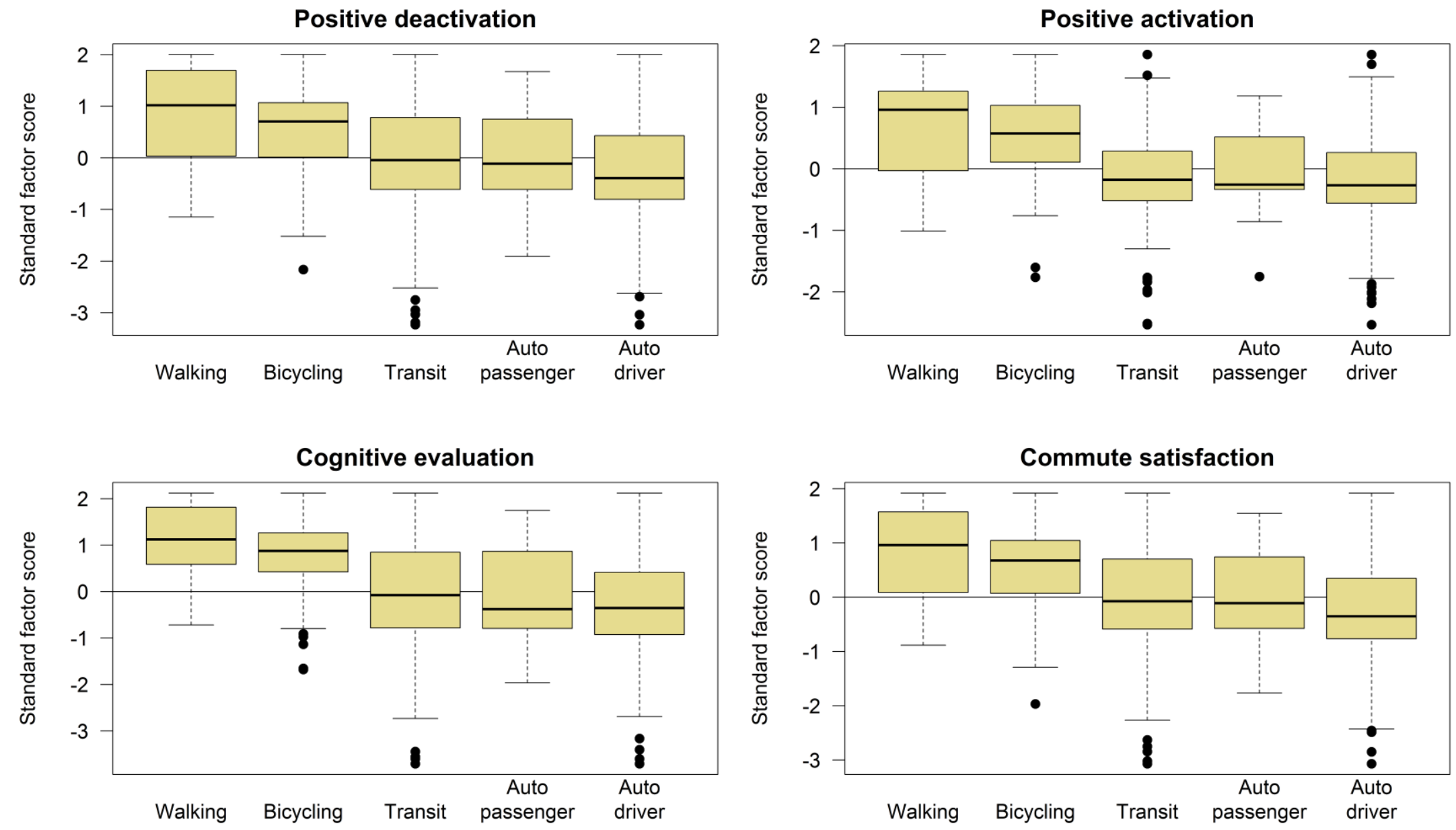

Figure 5.4 Box plots of STS factor scores by commute mode 
To examine other potential determinants of the STS, MIMIC models were estimated using the exogenous trip and traveler characteristics shown in Table 5.12 to predict the three STS latent variables found in the CFA ("Positive deactivation," "Positive activation," and "Cognitive evaluation"); a separate MIMIC model predicted just the higher-order "Commute satisfaction" concept. Model estimation results for the regression portions of the MIMIC models are presented in Table 5.12; only variables with marginally significant associations $(p \leq 0.10)$ are presented. (Full model results may be obtained by contacting the author.) The trip and traveler characteristics explained between a third and a half of the variance in the latent variables, with lower fit for the PD construct $\left(R^{2}=0.34\right)$ and higher fits for the PA $\left(R^{2}=0.46\right), \mathrm{CE}\left(R^{2}=0.49\right)$, and overall "Commute satisfaction" $\left(R^{2}=0.47\right)$ factors. 
Table 5.12 MIMIC model results for the STS

\begin{tabular}{|c|c|c|c|c|c|c|c|c|}
\hline \multirow[t]{2}{*}{$N=621$} & \multicolumn{2}{|c|}{$\begin{array}{l}\text { PD: Positive } \\
\text { deactivation }\end{array}$} & \multicolumn{2}{|c|}{$\begin{array}{r}\text { PA: Positive } \\
\text { activation }\end{array}$} & \multicolumn{2}{|c|}{$\begin{array}{r}\text { CE: Cognitive } \\
\text { evaluation }\end{array}$} & \multicolumn{2}{|c|}{$\begin{array}{r}\text { Commute } \\
\text { Satisfaction }\end{array}$} \\
\hline & $B$ & $p$ & $B$ & $p$ & $B$ & $p$ & $B$ & $p$ \\
\hline \multicolumn{9}{|l|}{ Trip characteristics } \\
\hline Mode: Walk & 0.520 & $0.068 \sim$ & 0.850 & $0.012 *$ & 0.722 & $0.008 *$ & 0.742 & $0.012 *$ \\
\hline Bicycle & 0.033 & 0.855 & 0.504 & $0.010^{*}$ & 0.250 & 0.135 & 0.254 & 0.131 \\
\hline Transit & 0.369 & $0.025 *$ & -0.141 & 0.360 & 0.187 & 0.195 & 0.189 & 0.192 \\
\hline Auto, passenger & 0.189 & 0.393 & -0.005 & 0.981 & 0.182 & 0.392 & 0.159 & 0.436 \\
\hline Travel time (minutes) & -0.010 & $0.002 *$ & -0.002 & 0.451 & -0.010 & $0.002 *$ & -0.009 & $0.004 *$ \\
\hline \multicolumn{9}{|l|}{ Traveler socio-demographics } \\
\hline Gender: Female & -0.175 & $0.079 \sim$ & -0.254 & $0.011 *$ & -0.103 & 0.281 & -0.166 & $0.080 \sim$ \\
\hline Disability & -0.372 & $0.052 \sim$ & -0.336 & $0.084 \sim$ & -0.423 & $0.043 \sim$ & -0.419 & $0.031 *$ \\
\hline Student & -0.258 & 0.116 & -0.082 & 0.640 & -0.331 & $0.034 *$ & -0.280 & $0.079 \sim$ \\
\hline Income: $150 \mathrm{k}+$ & 0.146 & 0.340 & 0.188 & 0.237 & 0.248 & $0.087 \sim$ & 0.223 & 0.126 \\
\hline \# commute days & 0.126 & $0.050 \sim$ & 0.013 & 0.827 & 0.022 & 0.705 & 0.055 & 0.349 \\
\hline Self-employed & 0.316 & 0.240 & 0.474 & $0.078 \sim$ & 0.411 & $0.100 \sim$ & 0.425 & 0.105 \\
\hline \multicolumn{9}{|l|}{ Traveler perceptions } \\
\hline \multicolumn{9}{|l|}{ Typical travel time: } \\
\hline Dissatisfied & -0.226 & $0.041 *$ & -0.221 & $0.050 *$ & -0.370 & $0.000 *$ & -0.320 & $0.002 *$ \\
\hline \multicolumn{9}{|l|}{ Travel usefulness: } \\
\hline Mostly wasted & -0.551 & $0.003 *$ & -0.644 & $0.000 *$ & -0.530 & $0.003 *$ & -0.605 & $0.001 *$ \\
\hline Somewhat wasted & -0.143 & 0.300 & -0.200 & 0.129 & -0.055 & 0.667 & -0.120 & 0.339 \\
\hline Somewhat useful & 0.094 & 0.471 & 0.109 & 0.393 & 0.357 & $0.004 *$ & 0.247 & $0.042 *$ \\
\hline Mostly useful & 0.537 & $0.004 *$ & 0.518 & $0.004 *$ & 0.692 & $0.000 *$ & 0.660 & $0.000 *$ \\
\hline \multicolumn{9}{|l|}{ Model fit statistics $\left(R^{2}\right)$} \\
\hline Trip only & 0.162 & & 0.249 & & 0.287 & & 0.258 & \\
\hline Socio-demo only & 0.124 & & 0.156 & & 0.151 & & 0.145 & \\
\hline Perceptions only & 0.220 & & 0.285 & & 0.362 & & 0.342 & \\
\hline$R^{2}$ overall & 0.342 & & 0.455 & & 0.485 & & 0.472 & \\
\hline
\end{tabular}

Statistical significance: $*=p \leq 0.05, \sim=p \leq 0.10$.

Variables having coefficients with $p>0.10$ for all factors are not shown.

After controlling for other factors, commute mode remained a significant factor. People walking had higher travel well-being and higher scores on all STS factors than auto drivers. Overall "Commute satisfaction" scores were also higher for bicycle, transit, and auto passenger commuters than for auto drivers, but these differences were not statistically significant. Transit riders had higher PD scores and bicycle commuters had higher PA scores. Travel time also appeared to be a determinant of the STS: Longer duration trips were rated more negatively overall, although not for the PA construct. Nonlinear 
(logarithmic, quadratic) representations of travel time did not significantly improve model fit. A model (not shown) in which travel time was interacted with commute mode found that its negative effect was relatively constant across modes. Interestingly, significant residual modal effects disappeared when controlling for mode-specific travel times.

Only a few socio-demographic traveler characteristics appeared to be determinants of the STS. Overall, women, students, and people with disabilities all reported significantly lower levels of well-being and satisfaction with their commutes. People who were selfemployed had higher STS scores, and more-frequent commuters reported higher levels of less-active positive affect. Traveler socio-demographics contributed a smaller portion of explained variance in the latent variables than variables of other types.

Instead, traveler perceptions dominated: Several variables were significantly associated with the STS and its three constructs. People who were dissatisfied with their typical commute travel times also reported lower levels of travel well-being. The subjective travel usefulness measure (Chapter 4) was also a significantly factor: Travelers with more useful commutes also had higher STS scores.

\subsubsection{Travel affect}

Modal differences appeared to influence commuters' responses to questions about travel affect. Figure 5.5 displays the five travel affect items that were most frequently reported to have been felt "at least a little" by travelers of different modes; green adjectives are positive, and red ones are negative. Travelers by all modes frequently reported feeling Alert and Attentive; this finding is not surprising since these indicate attention to and engagement with the traveling task. Walk and bicycle commuters overwhelmingly reported 
other positive attributes, especially feeling Active and Strong, but also Inspired for walking and Calm for bicycling. For motorized travelers, the only other positive emotion reported by at least half the respondents was Calm. (Some transit commuters also reported feeling Active, presumably because of nonmotorized access/egress trips.) Instead, negative adjectives started to appear, including feeling Stressed, Frustrated, or Nervous, although negative emotions were still less frequent than positive ones across all modes.

Travel affect (Walking)

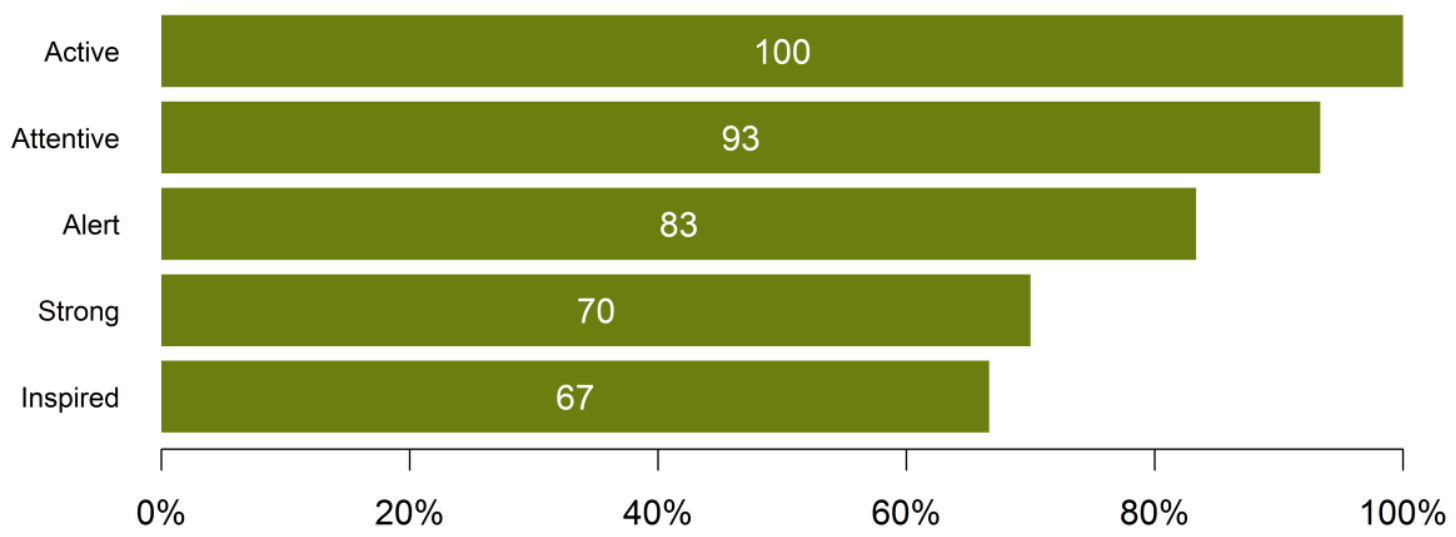

Travel affect (Bicycling)

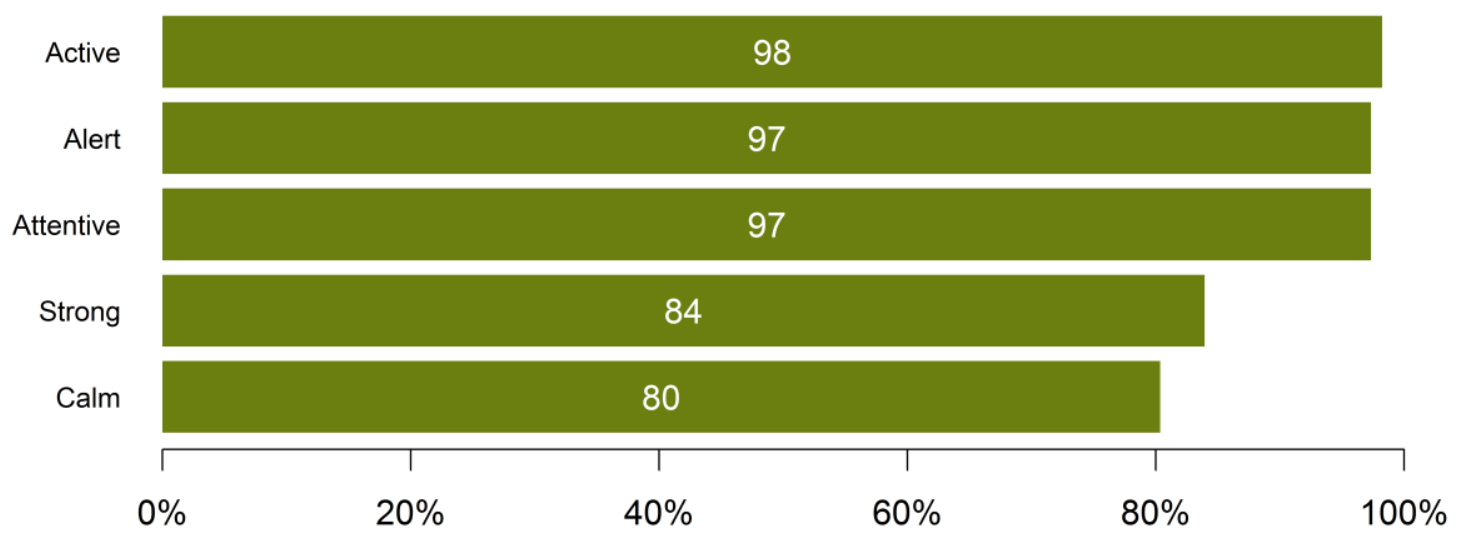




\section{Travel affect (Transit)}

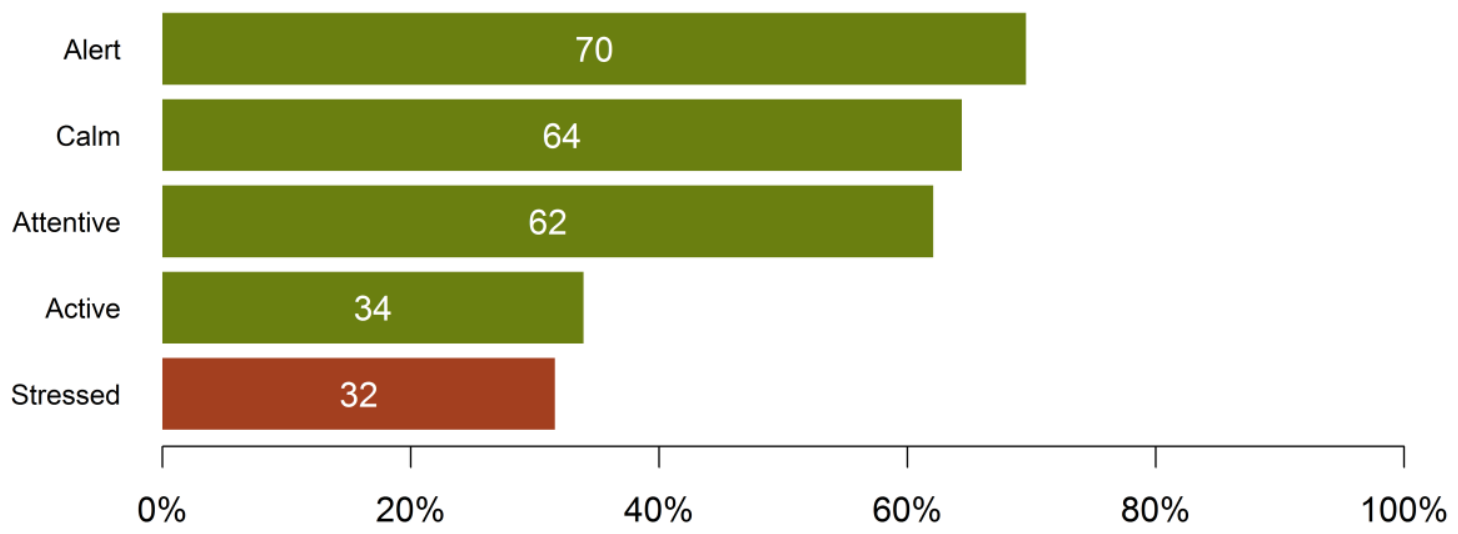

Travel affect (Auto passenger)

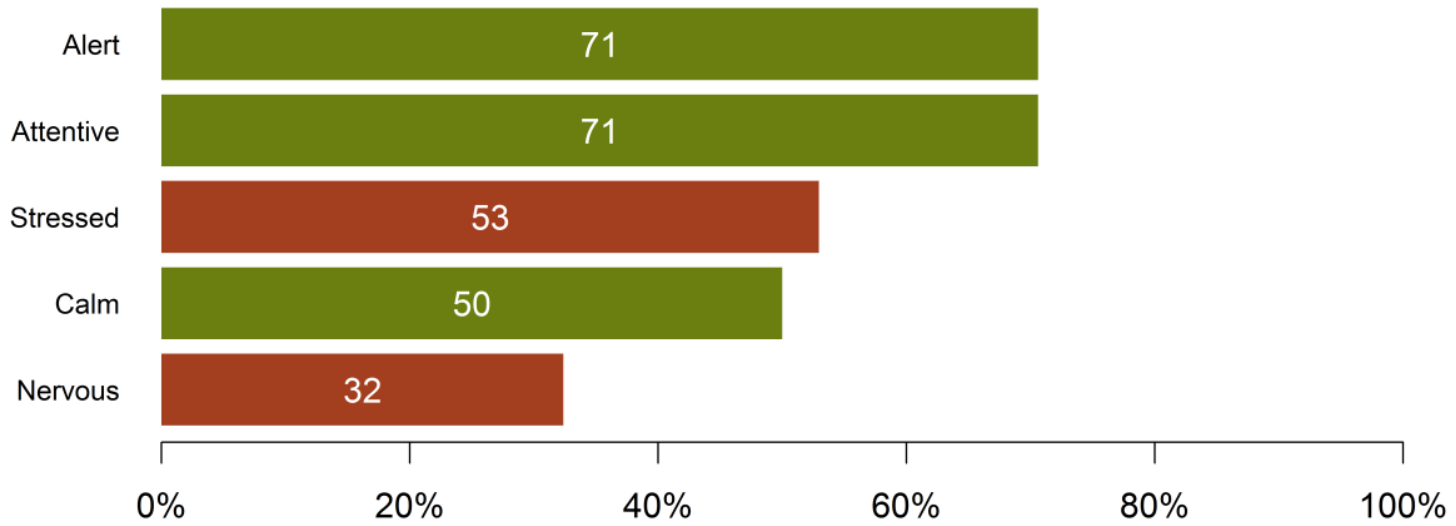

Travel affect (Auto driver)

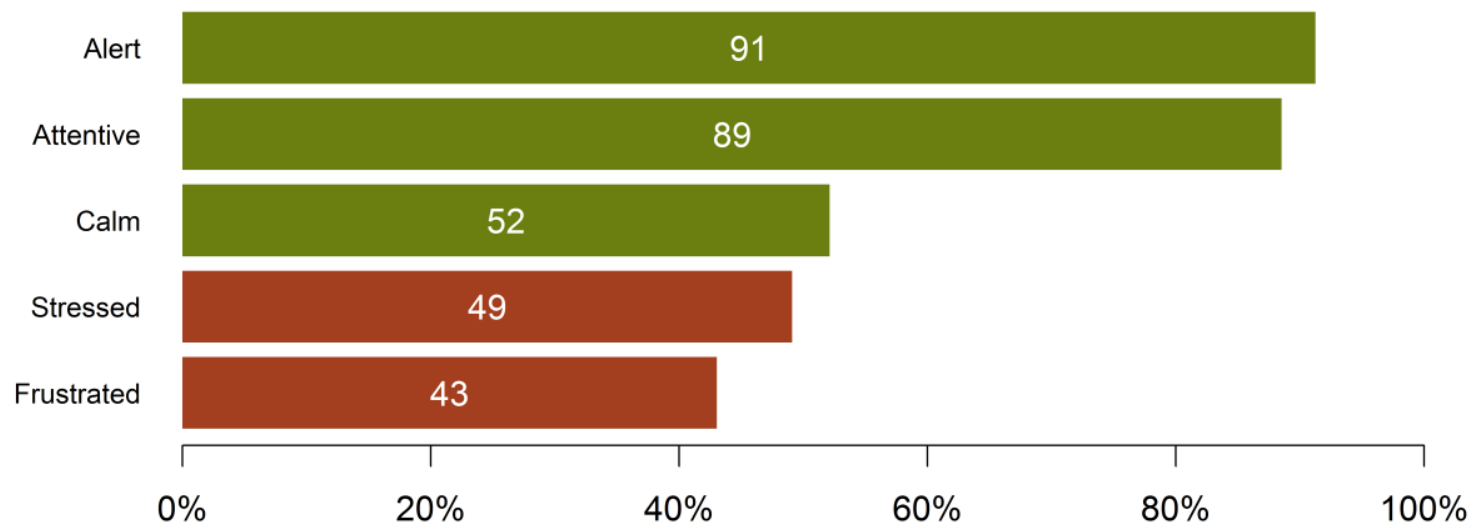

Figure 5.5 Frequently reported travel affect items by commute mode 
To examine modal differences more systematically, standardized factor scores of the four travel affect latent variables from the CFA in Figure 5.2 were analyzed; box-andwhisker plots for each factor by mode are shown in Figure 5.6. Ratings of "Distress" were low overall but slightly higher for auto drivers and bicycle riders, with some positive outliers especially for transit riders and auto drivers. Scores on the "Fear" factor were also low but positively skewed, although bicycle commuters exhibited much higher levels on average. Ratings of "Attentiveness" showed high variability within modes, but overall, bicycle commuters scored higher on this construct and transit riders and auto passengers scored lower. The "Enjoyment" factor displayed the biggest qualitative modal differences, with travelers rating commutes by nonmotorized modes to be much more positive than those by motorized modes.

Beyond these apparent significant differences among commute modes, other factors may influence travel affect. To investigate other potential determinants, a MIMIC model predicted the four travel affect factors by the same exogenous trip and traveler characteristics as were used to analyze the STS. See Table 5.13 for significant estimation results of the regressions; full results are available from the author. Model goodness-of-fit statistics varied across the factors: Higher fits were found for the positive constructs ("Enjoyment" $R^{2}=0.61$; "Attentiveness" $R^{2}=0.47$ ) than for the negative constructs ("Distress" $R^{2}=0.29$; "Fear" $R^{2}=0.26$ ). 

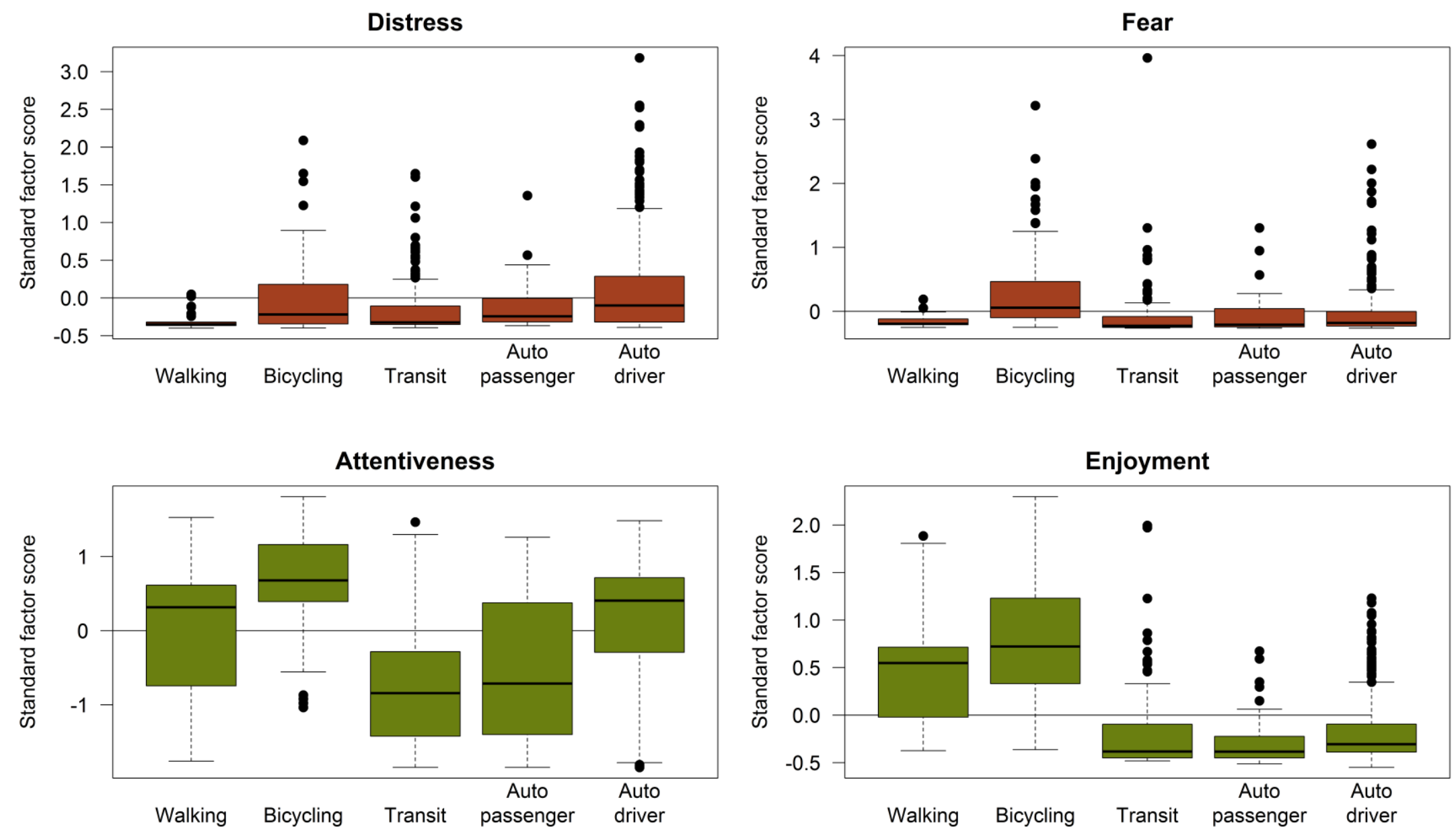

Figure 5.6 Box plots of travel affect factor scores by commute mode

용 
Table 5.13 MIMIC model results for travel affect

\begin{tabular}{|c|c|c|c|c|c|c|c|c|}
\hline \multirow[t]{2}{*}{$N=645$} & \multicolumn{2}{|c|}{ Distress } & \multicolumn{2}{|r|}{ Fear } & \multicolumn{2}{|c|}{ Attentiveness } & \multicolumn{2}{|c|}{ Enjoyment } \\
\hline & $B$ & $p$ & $B$ & $p$ & $B$ & $p$ & $B$ & $p$ \\
\hline \multicolumn{9}{|l|}{ Trip characteristics } \\
\hline Mode: Walk & -0.495 & $0.002 *$ & -0.108 & 0.460 & -0.547 & $0.028 *$ & 1.061 & $0.001 *$ \\
\hline Bicycle & 0.108 & 0.491 & 1.152 & $0.000 *$ & 0.183 & 0.281 & 1.360 & $0.000 *$ \\
\hline Transit & -0.514 & $0.000 *$ & -0.148 & 0.238 & -1.320 & $0.000 *$ & -0.115 & 0.359 \\
\hline Auto, passenger & -0.410 & $0.021 *$ & 0.132 & 0.530 & -1.129 & $0.000 *$ & -0.241 & 0.148 \\
\hline Travel time (minutes) & 0.011 & $0.000 *$ & 0.009 & $0.018 *$ & 0.003 & 0.383 & 0.003 & 0.345 \\
\hline \multicolumn{9}{|l|}{ Traveler socio-demographics } \\
\hline Gender: Female & -0.052 & 0.597 & -0.008 & 0.926 & -0.175 & $0.076 \sim$ & -0.212 & $0.028 *$ \\
\hline $\begin{array}{l}\text { Race/ethnicity: Hispanic/ } \\
\text { non-white/multiple }\end{array}$ & 0.108 & 0.414 & 0.357 & $0.042 *$ & 0.079 & 0.529 & 0.192 & 0.167 \\
\hline Education: No degree & 0.095 & 0.463 & -0.057 & 0.690 & 0.241 & $0.090 \sim$ & 0.021 & 0.871 \\
\hline \# children (age $\leq 16$ ) & -0.121 & $0.042 *$ & 0.000 & 0.996 & -0.069 & 0.240 & -0.014 & 0.809 \\
\hline \# seniors (age 65+) & -0.090 & 0.546 & -0.219 & $0.026 *$ & -0.226 & 0.188 & -0.170 & 0.214 \\
\hline Income: $\quad \$ 0-50 \mathrm{k}$ & -0.228 & 0.202 & 0.123 & 0.604 & 0.191 & 0.323 & 0.351 & $0.064 \sim$ \\
\hline Missing & -0.011 & 0.965 & 0.233 & 0.452 & 0.430 & $0.069 \sim$ & 0.077 & 0.734 \\
\hline Multifamily home & 0.220 & 0.125 & 0.326 & $0.085 \sim$ & -0.001 & 0.996 & 0.142 & 0.267 \\
\hline \# cars & -0.016 & 0.766 & 0.014 & 0.703 & 0.011 & 0.849 & 0.094 & $0.083 \sim$ \\
\hline \multicolumn{9}{|l|}{ Traveler perceptions } \\
\hline \multicolumn{9}{|l|}{ Typical travel time: } \\
\hline Dissatisfied & 0.358 & $0.000 *$ & -0.043 & 0.599 & 0.114 & 0.307 & -0.092 & 0.317 \\
\hline Ideal travel time & -0.009 & $0.093 \sim$ & -0.010 & $0.092 \sim$ & 0.006 & 0.298 & 0.006 & 0.249 \\
\hline \multicolumn{9}{|l|}{ Travel usefulness: } \\
\hline Mostly wasted & 0.700 & $0.003 *$ & 0.351 & $0.087 \sim$ & -0.136 & 0.450 & -0.240 & $0.044 *$ \\
\hline Somewhat wasted & 0.194 & 0.210 & 0.071 & 0.617 & 0.085 & 0.559 & -0.143 & 0.184 \\
\hline Somewhat useful & -0.033 & 0.760 & -0.057 & 0.691 & 0.196 & 0.169 & 0.072 & 0.483 \\
\hline Mostly useful & -0.155 & 0.197 & -0.359 & $0.030 *$ & 0.326 & $0.060 \sim$ & 0.458 & $0.003 *$ \\
\hline \multicolumn{9}{|l|}{ Model fit statistics $\left(R^{2}\right)$} \\
\hline Trip only & 0.146 & & 0.134 & & 0.385 & & 0.509 & \\
\hline Socio-demo only & 0.077 & & 0.112 & & 0.198 & & 0.204 & \\
\hline Perceptions only & 0.176 & & 0.015 & & 0.045 & & 0.319 & \\
\hline$R^{2}$ overall & 0.293 & & 0.257 & & 0.471 & & 0.612 & \\
\hline
\end{tabular}

Statistical significance: $*=p \leq 0.05, \sim=p \leq 0.10$.

Variables having coefficients with $p>0.10$ for all factors are not shown.

Commute mode remained a significant factor after controlling for other trip and traveler characteristics, especially for positive affect. Travel by nonoperating modes (walk, transit, auto passenger) was rated significantly lower on "Distress" than when commuters had to operate a vehicle (bicycle or auto). "Attentiveness" by travelers using the same nonoperating modes was also lower. Bicycle commuters' high ratings on the "Fear" 
construct, and nonmotorized travelers" high levels of "Enjoyment" remained statistically significant. Travel time was positively associated with negative emotions but was not associated with positive emotions. In a model with travel time segmented by mode (not shown), this association appeared to be strongest for transit riders (in both negative constructs) and for auto drivers (only in "Fear"). When this travel time $\times$ mode interaction was included, the other modal effects diminished for negative constructs but did not disappear completely. Nonlinear specifications for travel time were tested but found to be not significant.

Few traveler socio-demographic attributes were consistently or even significantly associated with travel affect. Travelers with more children had lower "Distress" scores, and those living with more older adults had lower "Fear" scores. People reporting nonwhite, including mixed, racial/ethnic backgrounds and those living in multifamily housing indicated having higher levels of "Fear" on their commutes. Women were less likely to report positive affect than men. On the positive side, low-income travelers and those owning more cars scored higher on the "Enjoyment" factor.

Travel perceptions were also not strongly or consistently related to travel affect factors. People dissatisfied with their typical commute travel time were more likely to report items of "Distress." On the other hand, commuters whose ideal travel times were longer had lower scores on the two negative affect constructs. Travel usefulness, while not consistently significant, did appear to be associated with travel affect: Commuters viewing their trips as being more useful scored lower on the negative affect constructs and higher on the positive affect constructs. 


\subsubsection{Travel eudaimonia}

Differences across commute modes were also apparent for more eudaimonic aspects of travel well-being. The five most commonly reported items for each mode are shown in Figure 5.7. (All of these items are assumed to have contributed positively towards SWB.) Most walking and bicycling commuters checked multiple common items, while the most frequently selected for auto drivers were reported only about half of the time. The most common items for nonmotorized commuters were feelings of physical and mental health, including stress relief or viewing the commute as a buffer. There was some overlap between bicycle and transit riders, who both commonly viewed commuting as $A$ buffer between home and work or a way to express Environmental values. Comfort and Companionship were most common for auto passengers, while Control and Independence were more common among auto drivers. Several other items showed up prominently across multiple modes, including Freedom and A routine.

\section{Travel eudaimonia (Walking)}

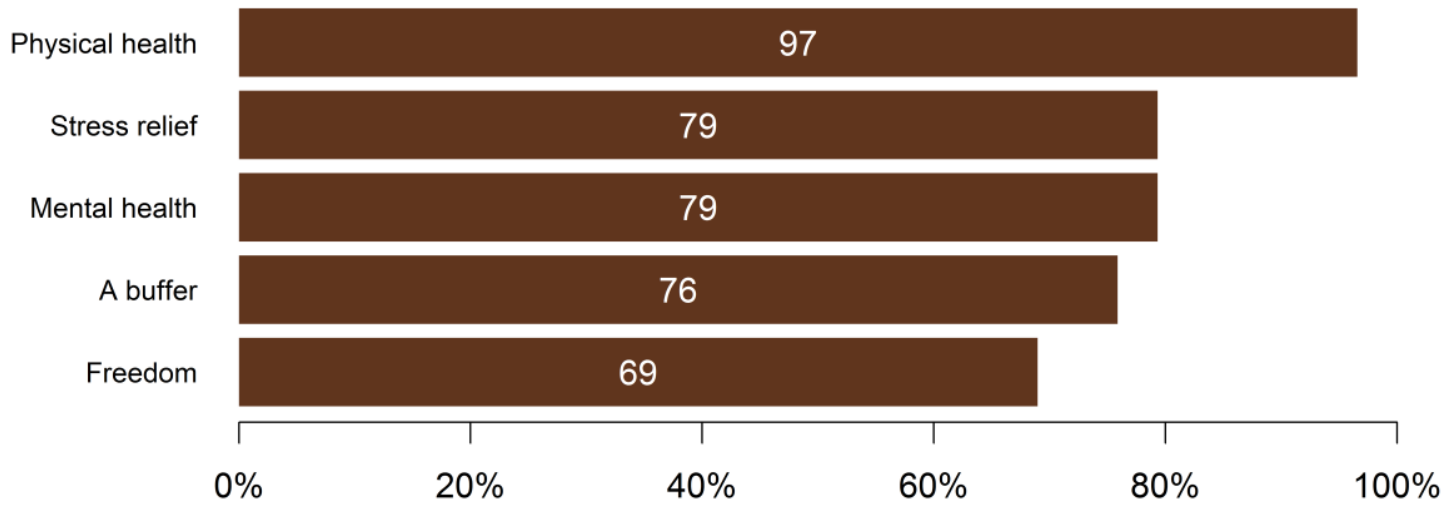


Travel eudaimonia (Bicycling)

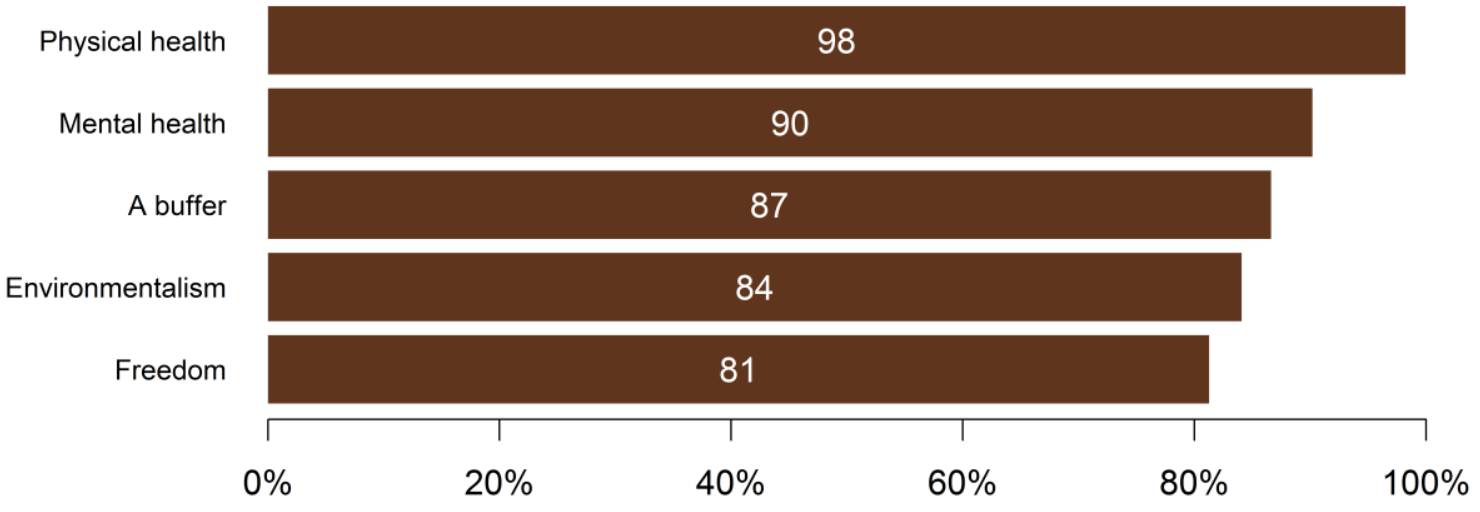

Travel eudaimonia (Transit)

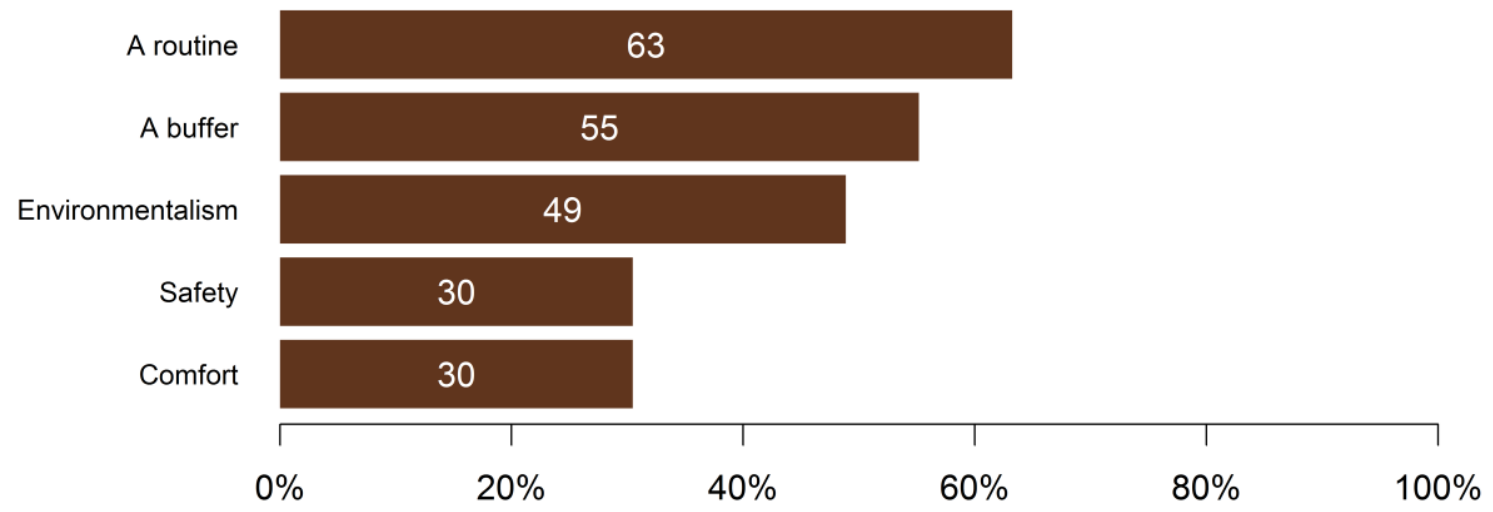

Travel eudaimonia (Auto passenger)

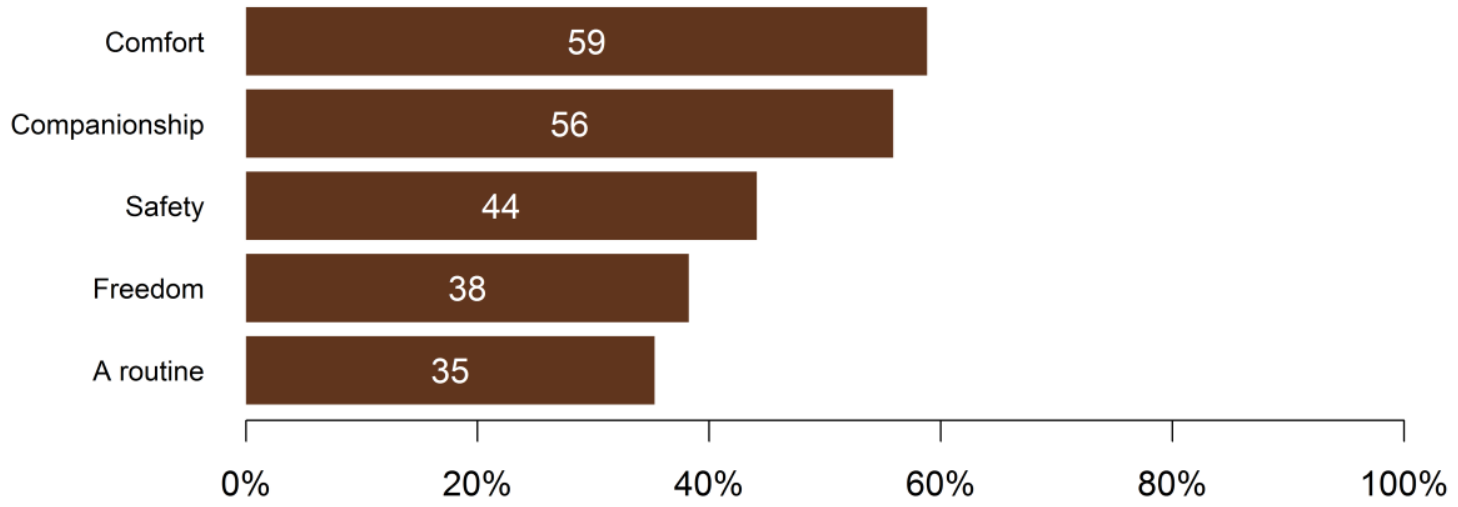




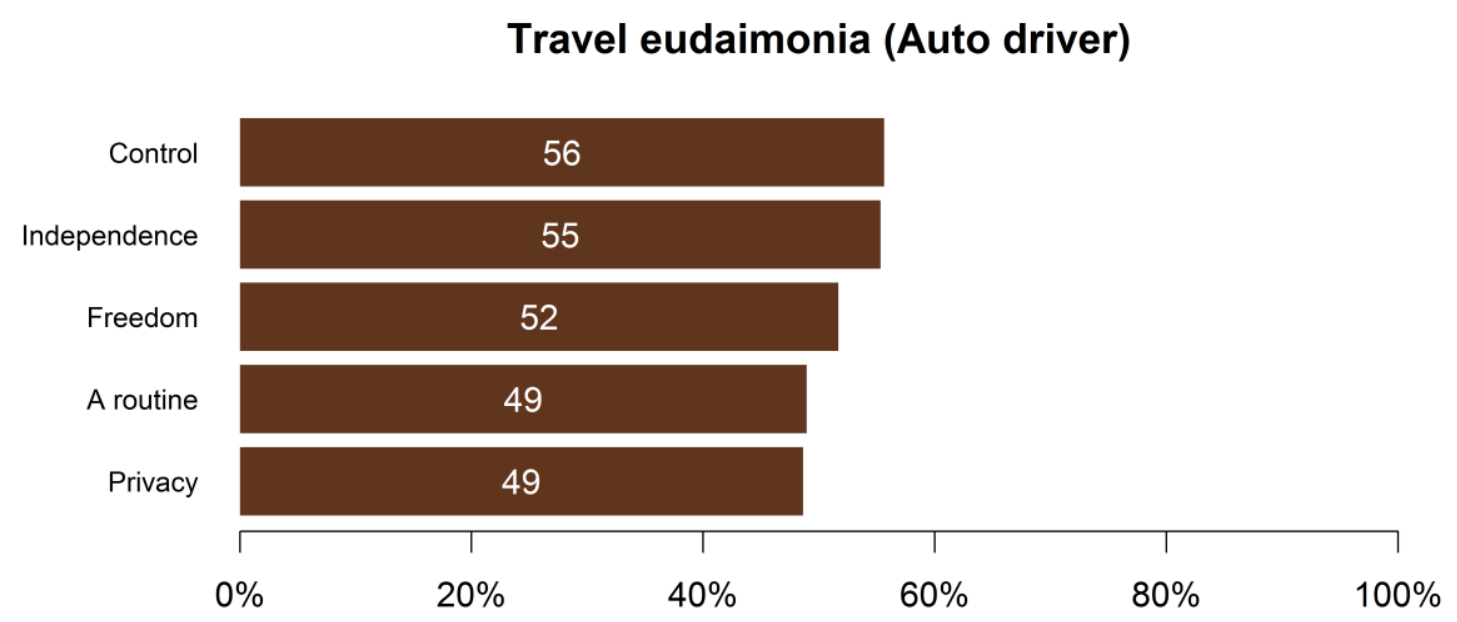

Figure 5.7 Frequently reported travel eudaimonia items by commute mode

Modal differences also appeared when considering the distribution of travel eudaimonia CFA factor scores, as shown by the box-and-whisker plots in Figure 5.8. On the "Autonomy" factor, modes in which travelers were captive to the decisions of other operators (transit riders and auto passengers) scored lower. Walking and bicycling commuters reported higher levels of "Confidence"; these modes also scored much higher on the "Health" construct. In contrast, modal differences were less pronounced for "Security": Auto travelers ranked slightly higher, and transit riders slightly lower, on average. 

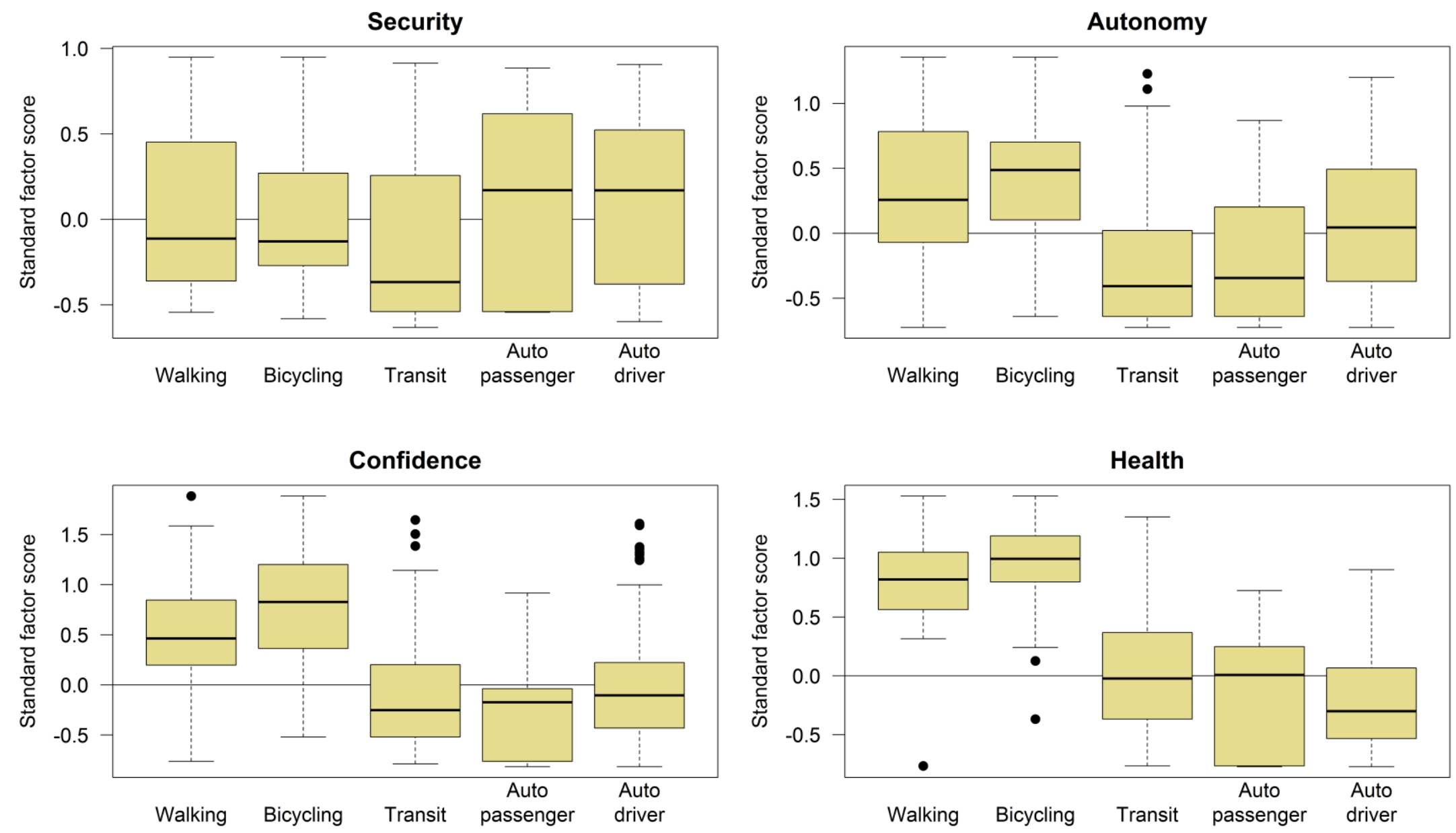

\section{Figure 5.8 Box plots of travel eudaimonia factor scores by commute mode}


A MIMIC model was estimated, using trip and traveler characteristics to predict the four latent variables representing travel eudaimonia. Table 5.14 presents abbreviated results for significant variables. Fit statistics were not quite as good as for travel affect and the STS, roughly in the one-quarter to one-third range of proportion of variance explained (“Autonomy" $R^{2}=0.35$; "Confidence" $R^{2}=0.33$; "Security" $R^{2}=0.22$ ). A significant exception was the "Health" construct: The exogenous variables actually explained most of its variance $\left(R^{2}=0.74\right)$. Note that in this MIMIC model, the modes and the travel usefulness dummy variables had to be collapsed due to empirical identification issues (zero cells). 


\section{Table 5.14 MIMIC model results for travel eudaimonia}

\begin{tabular}{|c|c|c|c|c|c|c|c|c|}
\hline \multirow[t]{2}{*}{$N=643$} & \multicolumn{2}{|c|}{ Security } & \multicolumn{2}{|c|}{ Autonomy } & \multicolumn{2}{|c|}{ Confidence } & \multicolumn{2}{|c|}{ Health } \\
\hline & $B$ & $p$ & $B$ & $p$ & $B$ & $p$ & $B$ & $p$ \\
\hline \multicolumn{9}{|l|}{ Trip characteristics } \\
\hline Mode: Walk/Bicycle & -1.057 & $0.000 *$ & 0.226 & 0.130 & 0.604 & $0.004 *$ & 1.441 & $0.000 *$ \\
\hline Transit & -0.591 & $0.001 *$ & -0.969 & $0.000 *$ & -0.080 & 0.718 & 0.391 & $0.001 *$ \\
\hline Travel time (minutes) & 0.001 & 0.825 & 0.000 & 0.892 & 0.004 & 0.291 & 0.005 & $0.027 *$ \\
\hline \# cotravelers & 0.159 & $0.032 *$ & -0.009 & 0.884 & -0.057 & 0.606 & 0.048 & 0.302 \\
\hline \multicolumn{9}{|l|}{ Traveler socio-demographics } \\
\hline \multirow{3}{*}{$\begin{array}{ll}\text { Age: } & 18-34 \text { years } \\
& 65+\text { years } \\
\text { Gender: } \text { Female }\end{array}$} & -0.246 & 0.169 & -0.278 & $0.064 \sim$ & -0.081 & 0.693 & 0.019 & 0.882 \\
\hline & 0.156 & 0.526 & 0.067 & 0.761 & 0.575 & $0.032 *$ & 0.317 & $0.060 \sim$ \\
\hline & -0.180 & 0.106 & -0.020 & 0.838 & -0.128 & 0.320 & -0.220 & $0.006 *$ \\
\hline \multicolumn{9}{|l|}{ Race/ethnicity: Hispanic/ } \\
\hline non-white/multiple & 0.112 & 0.471 & -0.084 & 0.532 & 0.469 & $0.004 *$ & 0.134 & 0.202 \\
\hline Education: Grad. deg. & -0.055 & 0.624 & -0.228 & $0.021 *$ & -0.278 & $0.045 *$ & -0.120 & 0.175 \\
\hline \# seniors (age 65+) & -0.081 & 0.707 & -0.489 & $0.003 *$ & -0.327 & 0.217 & -0.302 & $0.052 \sim$ \\
\hline \multirow{2}{*}{$\begin{array}{ll}\text { Income: } & \$ 0-50 \mathrm{k} \\
& \text { Missing }\end{array}$} & 0.062 & 0.765 & 0.143 & 0.463 & 0.553 & $0.014 *$ & 0.052 & 0.736 \\
\hline & -0.089 & 0.749 & -0.352 & $0.087 \sim$ & -0.240 & 0.409 & 0.095 & 0.640 \\
\hline \# bicycles & 0.013 & 0.687 & -0.008 & 0.756 & 0.064 & $0.036 *$ & -0.001 & 0.975 \\
\hline Car-share member & 0.151 & 0.240 & 0.188 & $0.091 \sim$ & 0.055 & 0.709 & 0.137 & 0.141 \\
\hline Transit pass & -0.006 & 0.957 & 0.006 & 0.954 & -0.111 & 0.425 & -0.176 & $0.054 \sim$ \\
\hline \multirow{2}{*}{$\begin{array}{l}\text { \# hours worked } \\
\text { Flexible work schedule }\end{array}$} & -0.022 & $0.003 *$ & -0.004 & 0.486 & -0.009 & 0.305 & -0.002 & 0.667 \\
\hline & 0.022 & 0.851 & 0.210 & $0.030 *$ & 0.038 & 0.791 & 0.026 & 0.753 \\
\hline \multicolumn{9}{|l|}{ Traveler perceptions } \\
\hline \multicolumn{9}{|l|}{ Typical travel time: } \\
\hline Dissatisfied & -0.634 & $0.004 *$ & -0.168 & 0.122 & -0.014 & 0.929 & -0.250 & $0.010 *$ \\
\hline \multirow{2}{*}{\multicolumn{9}{|c|}{$\begin{array}{l}\text { Teleportation: No } \\
\text { Travel usefulness: }\end{array}$}} \\
\hline & & & & & & & & \\
\hline Wasted & -0.177 & 0.264 & -0.155 & 0.251 & -0.076 & 0.704 & -0.236 & $0.039 *$ \\
\hline Useful & 0.261 & $0.061 \sim$ & 0.183 & 0.152 & 0.339 & $0.096 \sim$ & 0.304 & $0.004 *$ \\
\hline \multicolumn{9}{|l|}{ Model fit statistics $\left(R^{2}\right)$} \\
\hline Trip only & 0.086 & & 0.251 & & 0.187 & & 0.671 & \\
\hline Socio-demo only & 0.078 & & 0.156 & & 0.229 & & 0.312 & \\
\hline Perceptions only & 0.028 & & 0.065 & & 0.171 & & 0.453 & \\
\hline$R^{2}$ overall & 0.216 & & 0.349 & & 0.326 & & 0.739 & \\
\hline
\end{tabular}

Statistical significance: $*=p \leq 0.05, \sim=p \leq 0.10$.

Variables having coefficients with $p>0.10$ for all factors are not shown.

As with travel affect, the modal differences identified in the factor score plots of travel eudaimonia remained statistically significant in the MIMIC model. Walk, bicycle, and transit commuters felt significantly less "Secure" than auto commuters. "Autonomy" ratings were significantly lower for transit riders. Nonmotorized modes instilled higher "Confidence" and "Health" scores than did motorized modes, although transit riders 
reported more healthful commutes than auto travelers. Travel time was a significant and positive factor for only the "Health" construct. A travel time $\times$ mode interaction model (not shown) suggested this was mostly a positive association with "Health" of auto travel time; the interaction model also revealed a positive association between "Confidence" and walk/bicycle travel time. To test nonlinear effects, a quadratic travel time term was examined (model not shown); results suggested a minor but statistically significant quadratic (concave down) association between travel time and both "Autonomy" and "Confidence." Since there was no significant association detected for these factors when using a linear travel time specification, this finding should be investigated further. Finally, commuters traveling with other people reported feeling more "Secure."

Some socioeconomic and demographic characteristics of respondents were significantly associated with the various travel eudaimonia constructs. For "Security," commuters who worked longer hours had lower scores. Younger travelers, those with graduate degrees, and people living with a greater number of older adults had lower ratings for "Autonomy," while commuters who had flexible work schedules or car-share memberships had higher scores. Older commuters, those reporting nonwhite (or mixed) races or ethnicities, low-income travelers, and people owning more bicycles reported higher levels of "Confidence." Women, people with transit passes, and those living with older adults reported lower levels of "Health."

A few traveler perceptions were also related to travel eudaimonia, although these variables explained the smallest proportion of explained variance for all latent variables except "Health." Commuters dissatisfied with their typical travel times were less likely to report items related to "Security" and "Health." People who were not inclined to teleport 
to work were more likely to have high scores on "Health." The travel usefulness question appeared to have a positive effect on all constructs of travel eudaimonia, although this effect was not significant for "Autonomy."

\subsection{Discussion}

\subsubsection{Measuring commute well-being}

One of the primary objectives of this study was to develop more reliable and comprehensive measures of subjective well-being in the travel domain. Using the results of the EFA and CFA models, this goal was met, although improvements are always possible. The following sections discuss the strengths and weaknesses of the measurement models developed for the STS, travel affect, and travel eudaimonia.

\subsubsection{Satisfaction with Travel Scale}

Overall, this study confirmed the STS as a valid and reliable measure of hedonic SWB in the travel domain. The CFA model's good-to-adequate fit of the data and the emergence of a three-factor structure consistent with the PD/PA/CE distinctions found in other studies adds support to this conclusion. The new items and wordings adjusted for the U.S. context appear to have been successful, and this nine-item STS performed better-in terms of overall model goodness-of-fit, specific loadings, and reasonable item groupingsthan a seven-item version that was previously adapted to English-language and American contexts (Smith, 2017). 
As in most past applications of the STS, the results supported a three-factor STS structure: two affective constructs (positive deactivation, positive activation) and one cognitive construct. Notably, a two-factor structure (affective, cognitive) was not supported by the data considering its inadequate RMSEA value. However, the high correlation among the three latent variables, along with the goodness-of-fit of the secondorder construction of the STS, potentially suggests some discriminant validity problems in which the constructs are highly overlapping. In fact, the nine-item STS itself had a high degree of internal reliability (Cronbach's $\alpha=0.90)$.

These findings suggest that, although it may be a reliable overall measure of hedonic SWB in the travel domain, the STS may have difficulties distinguishing between the affective and cognitive aspects or at least along the activation dimension of core affect. Furthermore, with only nine items, the STS cannot illuminate many differences within the affect dimension of SWB, for example, the 11 specific affects (hostility, joviality, fatigue, etc.) measured by the PANAS-X expanded form (Watson \& Clark, 1994). More direct investigations of the affect dimension of SWB, such as are discussed in the following section, would be useful in this regard.

This study confirmed a few other limitations of the STS measurement model. Having more than two items load on the PD latent variable would have been desirable, although the internal reliability of this construct was acceptable (Cronbach's $\alpha=0.75$ ). More conceptually, some of the item-loading patterns were not a perfect match to hypotheses or intuition. For example, the Sad ... Happy item was intended to be a measure of valence, not activation, and the Trip was Displeasing ... Enjoyable item loading on CE is probably more of an affective consideration than a cognitive one. Finally, some of the 
results may have been affected by the order in which items were asked. Specifically, the four items loading on the CE factor were the last four items listed because they were longer statements. Although it makes sense that these are about a cognitive construct ("My trip was..." instead of "I was..."), these items could have grouped in part because of order effects due to item adjacency.

\subsubsection{Travel affect}

Despite only adequate goodness-of-fit statistics, the measurement model of travel affect was a success. All constructs had high internal reliability despite being measured by many items. They also were not strongly correlated with one another, suggesting discriminant validity. Additionally, the items loading on each concept were conceptually related among themselves, suggesting construct validity. In fact, there is considerable overlap among the items loading on the "Fear," "Attentiveness," and "Enjoyment" factors in this CFA and the items included in the "Fear," "Attentiveness," and "Self-Assurance" scales of PANAS-X (Watson \& Clark, 1994).

This study provides a substantial improvement to practice for measuring positive and negative affect from travel. The measurement model highlighted the distinctions between negative and positive affect, with modest correlations within and low correlations between each, resulting in a PANAS-like structure. It also suggests some of the limitations of the I-PANAS-SF instrument (Thompson, 2007) for measuring travel affect, particularly on the positive side: The two I-PANAS-SF items loading on "Enjoyment" (Inspired and Determined) were not the strongest loading items. However, this new scale's strength is that it builds upon these foundations, with question wordings and most items drawn from 
PANAS or PANAS-X. Although further research and validation are needed, this measurement of travel affect could help spur the development of a travel-specific instrument, potentially named PANTAS: the Positive and Negative Travel Affect Schedule.

That said, a few improvements to the CFA model are possible. In a travel context, "Attentiveness" is not necessarily a positive aspect; it could also reflect required attention paid to the traveling task so as to avoid collisions or injuries. Additionally, the "Enjoyment" factor was somewhat broad, with a large number of items measuring slightly different concepts. Some, like Proud, Inspired, and Bold, may be more about eudaimonia than about affect, although these are included in PANAS or PANAS-X, highlighting the overlap between these dimensions of SWB. It would be valuable to identify more unique positive affect constructs; this would require a larger sample of items and a more rigorous scale development process. Finally, the administration of this section of the survey utilized a categorical slider scale to add variety and retain respondents' attention. As a result, it was difficult to distinguish true responses of the default left end of the scale ("Very slightly or not at all") from skipped items. This analysis assumed a true response if at least one item was chosen in each block, but this decision could have introduced some biases. Future studies should be careful in designing online survey systems to avoid these measurement issues.

\subsubsection{Travel eudaimonia}

The measurement model of travel eudaimonia also illuminated useful patterns of SWB from travel. The four-factor CFA was a good fit to the data and suggested several 
nonaffective dimensions of travel well-being consistent with findings from past qualitative research on the psychosocial benefits and symbolic motivations for travel, including "Autonomy" and "Confidence." The "Health," "Confidence," and "Autonomy" constructs were particularly strong, with good internal reliability and conceptually consistent items.

Nevertheless, the measurement model for travel eudaimonia was not as clear-cut or as clean as the one for travel affect. As mentioned in the Results section, the "Security" measure was weaker, measured by only two items with slightly lower internal reliability than the other constructs. The latent variables remained highly correlated, and many items were removed due to high cross-loadings or other problems of discriminant validity. Together, this suggests that the travel eudaimonia factor structure measured somewhat overlapping constructs. Future work should investigate additional ways to better measure and distinguish between all of these concepts.

Part of the difficulty of measuring travel eudaimonia is related to the questionnaire itself. Unlike the five-to-seven point categorical scales used to measure travel affect and the STS, questions about travel eudaimonia were binary (checkbox), limiting the amount of variability in responses that could be captured. Part of this difficulty may be inherent: Travel eudaimonia could be a more challenging concept to measure than travel affect. Some of the components may be more implicit, or it may be more difficult to describe them in a way that people would consistently recognize. These are valuable points to consider in future work, although this study offers a good start. 


\subsubsection{Summary}

Table 5.15 below shows correlations between factor scores for the four concepts each of travel affect, travel eudaimonia, and the STS. Besides some strong correlations within constructs in the same dimension of SWB, a few others pairs were at least modestly correlated. There appeared to be substantial overlap (correlations above 0.60) between the affective measures of "Enjoyment" and the eudaimonic aspects of "Health" and "Confidence" (and, to a lesser extent, "Autonomy"). Indeed, as Figure 5.6 and Figure 5.8 make clear, people walking and bicycling reported higher scores on all of these constructs. Since the items used to measure these three concepts were conceptually distinct, this finding suggests that certain travel modes may tend to evoke these dimensions of SWB in conjunction with one another. Another result highlighted by Table 5.15 is that the STS components were moderately correlated $(>0.40)$ with some other measures of travel SWB: positively with "Enjoyment" and "Health" and negatively with "Distress." This finding suggests that the STS may be well suited to capture only relatively narrow types of positive and negative affect from travel. The unique measurement models of travel affect and eudaimonia offer the opportunity to use a more complex representation of the concept of SWB in the travel domain. 


\section{Table 5.15 Correlations between factor scores for travel SWB constructs}

\begin{tabular}{|c|c|c|c|c|c|c|c|c|c|c|c|}
\hline \multirow{3}{*}{ \# Construct } & & & & & & & & & & \multicolumn{2}{|c|}{ Correlations } \\
\hline & \multicolumn{4}{|c|}{ Travel affect } & \multicolumn{4}{|c|}{ Travel eudaimonia } & \multicolumn{3}{|c|}{ The STS } \\
\hline & 1 & 2 & 3 & 4 & 5 & 6 & 7 & 8 & 9 & 10 & 11 \\
\hline 1 Distress & & & & & & & & & & & \\
\hline 2 Fear & 0.56 & & & & & & & & & & \\
\hline 3 Attentiveness & 0.24 & 0.30 & & & & & & & & & \\
\hline 4 Enjoyment & -0.07 & 0.26 & 0.48 & & & & & & & & \\
\hline 5 Security & -0.08 & -0.06 & 0.19 & 0.20 & & & & & & & \\
\hline 6 Autonomy & -0.07 & 0.07 & 0.38 & 0.52 & 0.74 & & & & & & \\
\hline 7 Confidence & -0.08 & 0.15 & 0.38 & 0.66 & 0.41 & 0.83 & & & & & \\
\hline 8 Health & -0.16 & 0.13 & 0.28 & 0.65 & 0.31 & 0.64 & 0.85 & & & & \\
\hline 9 Positive deactivation & -0.48 & -0.21 & 0.08 & 0.42 & 0.25 & 0.34 & 0.37 & 0.43 & & & \\
\hline 10 Positive activation & -0.38 & -0.14 & 0.17 & 0.51 & 0.24 & 0.37 & 0.41 & 0.46 & 0.93 & & \\
\hline 11 Cognitive evaluation & -0.49 & -0.17 & 0.09 & 0.43 & 0.24 & 0.35 & 0.40 & 0.47 & 0.95 & 0.87 & \\
\hline 12 Commute satisfaction & -0.47 & -0.20 & 0.09 & 0.44 & 0.26 & 0.35 & 0.38 & 0.44 & 1.00 & 0.94 & 0.96 \\
\hline
\end{tabular}

\subsubsection{Determinants of commute well-being}

In addition to providing improved measures of commute well-being, this study documented additional evidence regarding potential determinants of SWB in the travel domain. The following sections discuss the travel behavior interpretations and implications of factors associated with the STS, travel affect, and travel eudaimonia.

\subsubsection{Satisfaction with Travel Scale}

Associations between trip characteristics and the STS were consistent with past research, including the finding that travelers reported lower well-being on longer trips. As has been documented in past research using the STS and numerous other studies, people bicycling and especially walking reported higher levels of travel SWB. This finding is consistent with a growing body of literature from both the health and psychology fields of the mental health and well-being benefits of physical activity (Biddle \& Mutrie, 2007; Penedo \& Dahn, 2005). Part of this association could be due to some sort of justification 
bias: thinking bicycling should be enjoyable and thus reporting it to be so. Alternatively, it could reflect the transportation environment in which walk/bicycle commuters live, one with usually more modal options from which to optimize one's commute well-being. This could result in some degree of self-sorting: People with options who like to walk and bike can do so, and people with options who prefer to drive can do so, but people who must drive or ride transit (because of longer distances or time constraints) but would prefer to walk or bike cannot and so report more dissatisfaction. Lower levels of well-being for auto and transit modes might reflect this discordance between travel preferences and travel options; it might also explain some of the negative association with travel time. However, given similar results for travel affect and eudaimonia, this finding suggests that the modal differences for STS do-at least in part-reflect true well-being benefits from physically active modes.

Other modal differences on STS factors were also relevant. Modes that scored higher on the PD construct involved fewer active driving and navigating tasks: walking, riding transit, and (although not significant) auto passengers. On the other hand, physically active modes (walking, bicycling) were the ones scoring highest on the PA factor. These modal groupings help explain why people walking reported significantly higher (and people driving significantly lower) levels of hedonic travel well-being: Walking belongs in both the nonoperational and physically active groups, while driving fits into neither.

The finding that women reported slightly lower levels of travel well-being is notable. This could be a reflection of remaining gendered societal differences in household roles, responsibilities, and divisions of labor (Sarmiento, 1998). For instance, if working women still take on more childcare and/or household maintenance responsibilities than 
men, they may experience greater time pressure (Bianco \& Lawson, 1998) and thus more stressful commutes. This finding could also reflect gender issues associated with residential location choice: Given growing two-worker households and the gender wage gap in the U.S. (Freedman \& Kern, 1997; MacDonald, 1999), households on average might choose a location to better optimize the male partner's commute, leaving women with less optimal commutes. Gender differences in fears over victimization and transportation security (Loukaitou-Sideris \& Fink, 2009) could be influencing women's lower reported travel well-being. There may also be unmodeled gender interactions, unobserved personality differences, or other factors that might partially explain this result.

Other findings make intuitive sense, such as the negative association with having a disability: Despite decades-long improvements in providing mobility for all, people with disabilities still may find traveling somewhat difficult. The negative association with travel time dissatisfaction is also not surprising. The finding that travel usefulness was positively related to the STS suggests that all of these measures may be picking up somewhat related or overlapping concepts. Alternatively, it could suggest a causal effect between travel activities and travel experiences, in which people feel more satisfied precisely because they are able to do things and make productive use of their travel time.

\subsubsection{Travel affect}

Modal differences on the positive side of travel affect are consistent with the literature on hedonic SWB in the travel domain. Overwhelming evidence suggests active travel is more positive; this study found that, on average, walk and bicycle commutes rated more than one standard deviation higher on the "Enjoyment" factor than auto or transit 
commutes. In fact, these modal (and other trip) differences contributed the majority of the explained variance in this and the other latent affect variables. As was discussed for the STS above, much of this difference may indeed be due to intrinsic affective benefits of physical activity, while some could be due to selection and sorting effects. The fact that nonoperator modes (walking, transit, auto passenger) saw lower ratings for "Attentiveness" is not surprising. Feeling Active or Attentive may be less about beneficial positive affect (a form of PUT) and more about required attention to the driving or bicycling task. In terms of core affect (Russell, 1980, 2003), "Attentiveness" may be more about activation than about valence.

On the negative side of travel affect, the MIMIC model highlighted interesting relationships with trip characteristics. The finding that longer trips saw more negative affect is consistent with previous research. It also suggests that the psychological stresses of commuting (potentially through congestion) or exposure to traffic can build up cumulatively. Studies on physiological commuting stress have documented positive associations between commute duration and levels of cortisol, a stress hormone (Evans \& Wener, 2006). Even after controlling for travel time, important modal differences in negative affect remained. The finding that bicycle commuters and auto drivers (but not passengers) reported higher levels of "Distress" suggests that the task of navigating roadways and interacting with other road users may be an important contributor to negative emotions during the commute. Bicycling's high score on the "Fear" factor (more than one standard deviation above other modes) likely had to do with commuters' fears about being vulnerable to injury when having to interact with high-speed and large-mass motor vehicles. Given that this high rating is for people who actually biked to work, and research 
suggests that existing bicyclists are more "fearless" (Dill \& McNeil, 2016) and that fear of traffic injuries is a strong deterrent to bicycling (Sanders, 2013; Schneider, 2013), this assessment might be even higher in the general (noncycling) population.

Interestingly, people of nonwhite (including multiple) races or ethnicities had higher scores for travel "Fear" on average, raising potential equity issues. Perhaps monetary constraints make it more difficult to move into a safer, lower-crime neighborhood; communities of color bear a disproportionate burden of traffic safety issues and unsafe roadways; or people in these circumstances have fewer options to travel by less frightening commute modes. On the other hand, perhaps these vulnerable populations may not feel as safe or secure when traveling for more social or societal reasons, such as a fear of victimization or discrimination by law enforcement or immigration authorities (Harris, 1999).

A few other results are worth discussing. The finding that women reported less positive affect echoes the discussion of similar results for the STS. Travel usefulness was associated with travel affect in similar and expected directions: Trips that were more useful were also rated as less negative and more positive. Again, making use of one's travel time may generate more positive feelings, or these questions could be imperfectly measuring slightly overlapping concepts. People with longer ideal travel times were also less likely to report negative affect. Presumably, this reflects a reverse directional relationship at a longer temporal scale: People who want longer commutes presumably like their typical commutes, so their most recent commute was likely less negative. This suggests a role for the consideration of travel affect in travel behavior choices. 


\subsubsection{Travel eudaimonia}

Analysis of potential determinants of travel eudaimonia also yielded interesting findings. The frequency analysis found far fewer items selected by auto commuters than by people walking or bicycling. This suggests more consistency in the experiences and/or evaluations of active mode users: Perhaps walk and bicycle commutes are more reliable than driving with respect to travel time and other experiential factors. Conversely, congestion or other negative experiences could generate more variability or less-positive assessments for auto drivers, or maybe the larger sample size of auto commuters naturally captured a wider range of experiences or a more heterogeneous population.

Other factors associated with each of the travel eudaimonic constructs are also interesting to discuss. The finding that nonauto-or personally exposed (Appleyard \& Ferrell, in press) — modes scored lower on the "Security" factor could suggest that private motor vehicles offer people more safety and privacy, considerations that may be related to the discomfort of interacting with strangers, fear of injury from traffic collisions, or fear of crime or victimization (Singleton \& Wang, 2014). The negative association of transit with "Autonomy" could be related to feelings of being captive to a schedule, route, or operator, with limited flexibility to adapt to changing needs or circumstances. The positive association with work-schedule flexibility supports this interpretation: Commuters with more leeway to arrange their work time likely have less schedule or time pressure and may be able to commute at more desirable times of day or have more modal options from which to choose.

People who commuted using active modes or who may be more likely to do so (as indicated by a positive association with household bicycle ownership) reported higher 
levels of "Confidence." This could indicate interesting but little-discussed benefits of bicycling: feeling adventurous or nonconformist (by using a "novel" or less common mode), being able to explore one's environment more closely, or being able to improve one's sense of self-efficacy. Surprisingly, the "Confidence" construct was positively associated with low-income and nonwhite race/ethnicity variables; this is in contrast to a positive association with "Fear." This finding remains unexplained and warrants further investigation.

Not surprisingly, physical activity seems to be highly valued (or at least frequently reported) by those who walked and bicycled, and - to a lesser extent—by transit commuters, who may get to or from transit by active modes. These "Health" benefits include not only physical health from exercise but also mental health benefits; as was discussed for the STS, this finding is consistent with a large literature on the health and well-being benefits of physical activity (Biddle \& Mutrie, 2007; Penedo \& Dahn, 2005). That women were less likely to report indicators of "Health" is consistent with similar findings from travel affect and the STS. Perhaps this (and some of those) results could reflect fewer women choosing active (and thus more healthy and positive) commutes; there remains a significant gender gap in bicycling in Portland (Singleton \& Goddard, 2016). The negative and positive associations with travel time dissatisfaction and travel usefulness echo similar findings for these variables, discussed in earlier sections.

\subsubsection{Summary}

Several findings that cut across the analyses of determinants of the STS, travel affect, and travel eudaimonia warrant a final mention. These analyses identified several 
ways to split modes that appear useful for understanding different dimensions of travel well-being. The first is to distinguish operating modes (bicycling, driving) — which require conscious attention to the task of navigating a vehicle-from nonoperating modes (walking, riding transit, and being an auto passenger). Although operating modes scored higher on "Attentiveness," they also had higher levels of "Distress" and lower PD scores. As discussed above, this suggests that having to operate a vehicle can be stressful and may degrade well-being. A second way to organize modes is into groups that are physically more active (walking and bicycling) or physically less active (transit, auto). Physically active modes scored higher on multiple constructs, including "Enjoyment," "Confidence," and "Positive activation." As discussed in previous sections, these findings could add to evidence about the mental health benefits of exercise and show that these benefits can also be obtained through physically active commutes. Together, these two modal distinctions could help to explain why some past literature has found travel well-being to be highest for walking/bicycling and lower for driving (as was found in this study for "Health," the CE construct, and overall "Commute satisfaction"): Walking is both a nonoperating and a physically active mode (a positive), while driving is both an operating and a physically inactive mode (a negative). Other modal findings - including the high ratings of "Fear" and low ratings of "Security" for bicycle commuters-likely relate to concerns over traffic safety (from fear of collision and injury) for this exposed mode (Appleyard \& Ferrell, in press).

Several results related to traveler characteristics also cut across multiple models. Women consistently reported lower levels of positive affect, eudaimonia, and overall hedonic well-being from the commute. As discussed above, this finding could reflect some 
effects of normative gender roles in society; however, much remains unexplained. It is consistent enough across the models in this study that further research should examine first whether it is purely an artifact of the sample, and (if not), why does it persist? Another repeated finding was the clear positive association between commuters' reports of travel usefulness and their travel well-being. As mentioned above, this result could suggest that making productive use of one's travel time could generate higher levels of SWB in the travel domain. However, more investigation is needed to examine this potential causal pathway.

Finally, the overall model goodness-of-fit statistics shed some light on the relative importance and relevance of various trip and traveler characteristics for explaining and predicting measures of travel well-being. (This discussion is based on interpretation of the relative contribution towards the total explained variance of each block of exogenous variables, as indicated by $R^{2}$ values.) Overall, attributes of the trip-particularly commute mode - were the most valuable variables for explaining travel SWB, especially for the more detailed measures of travel affect and eudaimonia. Traveler perceptions (about travel time satisfaction and travel usefulness) were moderately useful, depending on the construct, however they contributed the greatest amount of explained variance for components of the STS. Traveler socio-demographic characteristics performed the weakest, explaining usually less than $20 \%$ of latent variable variances, however they were relatively more useful in understanding some constructs of travel affect ("Fear," "Attentiveness") and most constructs of travel eudaimonia.

This summary confirms the significant role that the transportation experience plays in shaping subjective well-being in the travel domain, particularly due to intrinsic 
differences of each travel mode. This finding suggests that if travelers are aware of these modal differences in well-being, and have suitable options, they may make mode choices in order to (at least locally) maximize travel SWB. This study is also consistent with other research suggesting an important behavioral role for traveler attitudes and perceptions. More challenging for the study of SWB in the travel domain — and the positive utility of travel concept more generally—is the relative lack of explanatory power contributed by socio-demographic characteristics. These traveler attributes are more commonly, objectively, and (usually) easier to measure and collect data about than perceptions and especially the subjective assessments of travel well-being that were used in this survey. This result casts doubt that existing methods of data collection can be used to reliably predict travel well-being in a model. A fundamental shift in data collection efforts and perhaps modeling approaches is necessary in order to provide for a meaningful study of the connections between transportation and SWB.

\subsubsection{Limitations and future work}

There are a number of opportunities to improve and extend this work in future research. From a measurement perspective, this study demonstrated the potential value of more closely investigating different dimensions of hedonic and eudaimonic SWB from travel. Although the measurement models developed provide a solid beginning, a more rigorous scale development process (DeVellis, 2016) is warranted. Such a process should involve multiple qualitative and quantitative studies of broad and varied populations, the development and administration of much longer lists of potential items, and formal tests of reliability and validity, among other tasks. Additionally, this study focused solely on 
commute travel, but there may be different affect structures or symbolic motivations for nonwork travel, including for household maintenance or leisure purposes. Some evidence suggests these trips may actually be more positive than commuting (Mokhtarian \& Salomon, 2001). Performing invariance testing on the CFA structures of SWB in a larger multipurpose travel survey could illuminate these differences more clearly. These travel well-being constructs may also differ across modes; however, the sample sizes in this study may not have been sufficient to rigorously test for measurement invariance across different modes. This consideration should be investigated in larger future studies. A final measurement issue involves linking these findings to practice. This study measured but did not analyze travel liking, a measure of perhaps more affective SWB. (This is a task for future work.) Although not a perfect single measure, it has been somewhat widely used (e.g., Ory \& Mokhtarian, 2005) and is easily understood by respondents. If travel liking were closely related to some aspect of travel well-being, it could be used as a simple substitute or proxy measure in place of the more intensive questions about travel affect and eudaimonia in future travel surveys.

From the perspective of understanding factors associated with travel well-being, other opportunities are available. The "Distress" and "Fear" latent variables appeared to be positively skewed; using nonlinear link functions in the MIMIC model regressions could be a better approach to modeling these constructs and could improve their relatively low $R^{2}$ values. There may be other, more explanatory traveler characteristics that could be stronger determinants of travel well-being. For example, this study was not able to control for anticipatory effects, in which thinking about, preparing for, anticipating (or fearing) activities at the trip destination (work) may have affected emotions and travel motivations. 
On a larger time scale, people with higher overall SWB might also be more likely to have higher SWB in the travel domain, so it would be useful to control for travelers' satisfaction with life and in the home and work domains. Personality differences could also moderate affective responses to travel. Furthermore, there is evidence that activity participation during travel (travel-based multitasking) may positively influence both positive travel affect and cognitive evaluations of travel satisfaction (Ettema et al., 2012; Rasouli \& Timmermans, 2014a, 2014b; Rhee et al., 2013). The significant association of travel usefulness in many of the MIMIC models suggests that this may be true, but there could be better ways to control for it using more distinct concepts. The benefits of travel activities and travel experiences may be difficult to empirically disentangle.

A valuable area of research to pursue, and a logical next step to this work, is to investigate the extent to which travel SWB affects travel behavior; i.e., that expectations or assessments of travel well-being motivate or influence personal transportation decisions such as mode choice. Indeed, this work is presented in Chapter 6. Models linking travel well-being to mode choice are rare (see Chapter 2), likely because this task is very challenging both from a data collection and a modeling perspective. The measures of travel SWB used in this study were only concerned with the mode a traveler actually used for a recent commute trip. For mode choice analysis, attributes of all alternatives (including assessments of SWB for nonchosen modes) are necessary, whether collected directly or imputed using some sort of propensity modeling (Malokin et al., 2015). Important questions remain about the validity and comparability of retrospective vs. prospective and chosen vs. imagined assessments of travel (and general) well-being (Abou-Zeid \& BenAkiva, 2012; see Chapter 2). 
Even if the requisite data were collected or constructed, analysis is still challenging. Techniques for merging SEM and discrete choice models—-termed integrated choice and latent variable (ICLV) models—are becoming more common, yet challenges remain with respect to model run times, the incorporation of more than a couple of latent variables, sample size requirements, and measures of "good" fitting models (Bahamonde Birke, 2016; Vij \& Walker, 2016). Furthermore, most latent variables included are traveler-specific attitudes that do not vary across modes. The trip-relevant measures of travel SWB here are mode-specific perceptions (they vary across alternatives for each case), which may be more difficult to statistically accommodate. If these challenges could be addressed, behavioral interpretations and knowledge about the travel behavior impacts of SWB could be substantial.

Investigating the connections between travel SWB and SWB in other life domains could also provide important insights. Most applicable to this study is the way in which commuting can act as a time to relax and transition between home and work roles (Jain \& Lyons, 2008). Research in industrial-organizational psychology has demonstrated the benefits of home-work boundaries and especially psychological detachment in helping to mitigate the negative impacts of job stressors (e.g., Sonnentag, Kuttler, \& Fritz, 2010). Distancing oneself from work mentally (and physically) during nonwork time may reduce emotional exhaustion, increase cognitive hedonic SWB, and improve job performance. In fact, a moderate amount of psychological detachment— such as that provided by a 30minute commute - may be more beneficial than either higher or lower levels of detachment (Fritz, Yankelevich, Zarubin, \& Barger, 2010). Examining these relationships between to- 
work and from-work commute SWB, job performance, and satisfaction with life could prove interesting.

\subsubsection{Research implications and policy applications}

This study makes several contributions to research. By documenting reliable and conceptually meaningful ways to measure subjective well-being (including both hedonic and eudaimonic aspects) from travel, it makes an advancement in how these concepts can be investigated in travel behavior research. Future studies could adopt these instruments to measure travel affect and eudaimonia and use them-with a version of the Satisfaction with Travel Scale that has been validated for a U.S. context - to perform further testing or to analyze travel behaviors. Future research linking travel SWB to mode choice would be especially fruitful.

The relationships and potential determinants of travel SWB identified in these analyses also offer implications for transportation planning and policymaking. In particular, there may be transportation-related interventions that could improve the health and well-being of a population. A primary reason that walk and bicycle modes were so highly rated appears to be the physical and mental health benefits of exercising while on the commute. Thus, interventions aimed at increasing levels of walking and bicyclingincluding engineering efforts to make active travel safer and more feasible, such as through protected bike lanes or safer street crossings, and encouragement initiatives to make active travel more fun, friendly, and socially acceptable — might be able to substantially improve the subjective well-being of at least a subset of travelers. The high rating of "Fear" that existing bicycle commuters experienced (and that may be deterring potential cyclists) 
supports the widespread installation of protected bicycle infrastructure that eliminates many stressful roadway conflicts.

Although some of the modal differences could be due to self-selection effects, findings suggest there may be some people who are (for many reasons) captive to a particular mode or situation. Offering people more feasible modal options - by providing transit service that facilitates better access to jobs, or by providing safer streets and sidewalks upon which to walk and bike, for example — or improving the quality of service of existing modes - whether through more comfortable nonmotorized infrastructure, faster and less crowded transit vehicles, or less congested roadways — could increase travel wellbeing and decrease negative emotions experienced during the commute.

Finally, these findings have potential implications for understanding potential transportation futures. Much has been posited about the travel behavior impacts of fully autonomous vehicles (AVs), particularly how the ability to make productive use of travel time would likely decrease sensitivities to travel time and cost (reflected in reduced values of travel time savings), increase demand for automobile travel, and potentially offset any operational efficiency or roadway capacity gains obtained from vehicle-to-vehicle and vehicle-to-infrastructure communications (e.g., Childress et al., 2015). For the most part, these discussions have ignored potential impacts of AVs on travel experiences and wellbeing. The findings in this study suggest that if auto travelers no longer have to drive and operate a vehicle (thus, becoming more like auto passengers or transit riders), they may report lower levels of "Fear" and "Distress" while retaining higher ratings of "Protection." Reducing the stresses and fears of driving could increase travelers' well-being, which in turn could make auto travel marginally more attractive. On the other hand, travelers whose 
preferences for high levels of "Attentiveness" and "Freedom" are currently satisfied as drivers may find themselves experiencing more negative SWB in AVs if they no longer have the opportunity to operate a vehicle. Perhaps some of these displaced drivers may turn to bicycling to fulfill such desires. An understanding of mode-specific sensitivities to each of these well-being constructs (such as through the estimation of an ICLV mode choice model) could yield a better understanding of these potential mode shifts and well-being impacts. 


\section{Chapter 6 Mode choice \\ Exploring the Positive Utility of Travel and mode choice: Evidence from commuters in Portland, Oregon}

\subsection{Abstract}

The "positive utility of travel" (PUT) concept suggests two primary ways in which traveling can provide people with benefits besides reaching a destination: by facilitating travel-based multitasking through engagement in activities while traveling; and by improving subjective well-being (SWB) via positive emotions and symbolic expressions from the experience of traveling itself. Despite increased interest in these PUT-related aspects, little is known about their potential effects on travel behavior and, specifically, mode choice. This study is among the first to offer empirical evidence regarding the associations of travel activity and travel experience attributes of the PUT concept with travel mode choice. Notably, it uses a unique revealed preference dataset that measured these attributes not just for chosen modes but also for alternative modes. Data from a survey of about 550 commuters in the Portland, Oregon, metropolitan area were analyzed using an integrated choice and latent variable (ICLV) mode choice model. Results found significant associations between measures of travel-based multitasking and mode choice. In addition, a measure of SWB derived from the Satisfaction with Travel Scale (STS) was significantly and positively associated with commute mode choice. These findings are consistent with theory suggesting a link between the PUT concept and travel behavior. They also demonstrate that including direct measures of travel-based multitasking and SWB can help to explain a substantial portion of mode choice variations, suggesting that 
researchers should consider including these sorts of PUT measures in future travel behavior studies.

\subsection{Introduction}

Transportation studies frequently assume travelers seek to maximize the utilityor, more accurately, minimize the disutility — of traveling between locations. After all, a central tenet of mode choice modeling is that the travel time and cost coefficients should be negative and exhibit a narrowly defined relationship (Cambridge Systematics et al., 2012; Wardman, 1998). Transportation models assume people choose the mode or route that requires the minimum generalized cost (in minutes, dollars, or utils). Unfortunately, real world travel behaviors rarely adhere to these theoretical axioms. People may travel out of their way to enjoy pleasant scenery or for variety. While some people choose to commute by bicycle to get exercise or by train to get an early start on the workday, others drive fancy or powerful cars to feel in control or to express social status. Walking can be a time for reflection or preparation, improving mental health.

These instances are all examples of the positive utility of travel (PUT) concept (Mokhtarian \& Salomon, 2001; see Chapter 2). This idea suggests that travel time can be put to productive use and that physical, emotional, and symbolic benefits can result from the act of traveling. As described in the sections below, the PUT concept is closely related to other relevant issues in the travel behavior field, including travel-based multitasking and subjective well-being (SWB). It also has important potential implications for transportation planning and policymaking. These types of travel benefits could call into question estimates of economic willingness-to-pay measures that are central to the user benefits 
calculations of multimillion-dollar mobility-enhancing transportation projects (Mackie et al., 2001). A better understanding of the extent and effects of PUT measures could improve forecasts of walking, bicycling, and transit demand and increase the policy-sensitivity of travel demand modeling tools. Future increases in productivity (a central aspect of PUT) made possible by autonomous vehicles could dramatically change how people get around.

Despite these relevant implications, very little is known about the role of PUT aspects on travel behavior. Only a handful of studies have empirically examined various measures of PUT in conjunction with mode choice, and none has done so comprehensively. There is great potential for new knowledge and policy-relevant information from a more complete and theory-based investigation into the PUT concept. If attributes related to PUT play even a modest role in travel behavior choices, this is an important finding that could suggest behavioral interventions to nudge travelers at the margin towards more socially desirable transportation patterns. This research addresses these issues and gaps head-on by empirically examining the roles of travel activity and travel experience aspects of PUT in the travel mode choice decision.

\subsubsection{Research questions}

This study seeks to provide evidence towards answering the following broad research question. How does the positive utility of travel (PUT) concept affect travel behavior? More specifically: How do PUT measures impact commute mode choice? With respect to travel activity aspects: Are self-reported travel-based multitasking, activity participation, and travel usefulness metrics associated with imputed mode choice utility? Which activities, if any, are valued more highly in the commute mode choice decision 
process? With respect to travel experience aspects of PUT: Are self-reported measures of subjective well-being — specifically the Satisfaction with Travel Scale (STS)—associated with mode choice behavior? Overall, this study also seeks to answer the question: How behaviorally explanatory are these PUT factors in comparison to traditional level-ofservice attributes (travel time and cost) and traveler socio-demographic characteristics?

The research presented herein answers these questions by analyzing an integrated choice and latent variable (ICLV) model of mode choice, utilizing a 2016 survey of commuters in Portland, Oregon. This chapter is structured as follows: First, the PUT concept is described in more detail, including distinctions between travel activities and travel experiences. This literature review also summarizes findings from the few studies that have looked at PUT measures in a mode choice context. Next, the data and methods are described. Results of the ICLV model estimation process are then presented. Finally, the results and implications of this study are discussed, including opportunities for future work.

\subsection{Literature review}

\subsubsection{Positive Utility of Travel (PUT)}

The concept known as the positive utility of travel (PUT) stands in contrast to traditional theories and methods that drive travel behavior research and travel demand analysis. The prevailing transportation paradigm - particularly as derived from economic principles-presumes that people travel simply as a means to a greater end (going somewhere) and that travel time is a disutility to be minimized whenever possible. A 
corollary, and the basis for the activity-based approach to travel analysis (e.g., Kitamura, 1988; Pas, 1985), is that travel demand is derived from the demand for spatially separated activities. Theory suggests that the subjective value of a marginal reduction in travel time (also known as the value of travel time savings) can never be negative (Hess et al., 2005).

The PUT notion pokes holes in these universal assumptions, supposing instead that - for some people and in some situations - travel can provide benefits and be motivated by factors beyond simply reaching activity destinations. It unites several concepts relevant to travel behavior: utility maximization, motivation theory, satisfaction and subjective well-being, and multitasking, among others. While traditional approaches to mode choice analysis are rooted in microeconomic random utility maximization (RUM) theory (McFadden, 2001a), psychological approaches to utility are more broadly associated with aspects of pleasure, satisfaction, happiness, and well-being (Nordbakke \& Schwanen, 2014). Motivation theory, also a key thread in the field of psychology (Ryan \& Deci, 2000), suggests that intrinsic motivations can drive human behavior because of an enjoyment of the activity (in this case, travel) itself.

In the travel behavior research realm, these ideas have been raised periodically through the years in an attempt to better explain some of the observed variations and inconsistencies in personal transportation behaviors. Early work applying microeconomic time allocation theory to travel time valuation mentioned the possibility that travel time could be pleasant or productive (Becker, 1965; Evans, 1972; Johnson, 1966), generating a positive utility associated with travel time among "those who travel for travel's sake" (Oort, 1969, p. 283). Reichman (1976) wondered if transportation could "fulfill some ends in itself" (p. 148), and Hupkes (1982) discussed an "intrinsic utility" of traveling. 
Synthesizing these and other sources in a series of two influential papers, Salomon and Mokhtarian (1998; Mokhtarian \& Salomon, 2001) further articulated the concept of a positive utility of travel in their description of "an intrinsic drive for mobility" (p. 130). Their work brought increased attention to the PUT concept, leading to a special issue on the subject in the journal Transportation Research Part A: Policy and Practice (Mokhtarian, 2005). Recently, a thorough review article by Mokhtarian, Salomon, and Singer (2015) examined a variety of potential reasons for traveling in more detail, many of which are closely tied to PUT aspects.

As described in Chapter 2, a positive utility of travel (PUT) can be defined as any benefit(s) accruing to a traveler through the act of traveling. Building upon the work of Mokhtarian and Salomon (2001), who first described the "tripartite nature of the affinity for travel" (p. 701), Chapter 2 goes on to outline three components of the PUT concept:

1. Destination activities: Benefits from reaching a destination with activity potential;

2. Travel activities: Benefits from using travel time for activity participation (travelbased multitasking); and

3. Travel experiences: Benefits from the experience of traveling, including from:

a. Affective (hedonic) enjoyment of the travel experience, and

b. Symbolic (eudaimonic) expressions or fulfillment from the travel experience.

Destination activities are examples of the derived-demand paradigm and the activity-based travel analysis approach, which assumes people travel in order to conduct activities at locations separated in space. As destination activities are the traditional focus of travel behavior research, they are not of primary interest to this study. Instead, this 
analysis centers on the travel activity and travel experience components, as described in more detail in the following subsections.

\subsubsection{Travel activities}

One potential means of generating a PUT is by making use of one's travel time by conducting other activities. These travel activities presumably reduce the disutility of traveling by putting travel time to "productive" use (Lyons \& Urry, 2005), although they may be less likely than travel experience factors to generate a new trip (Mokhtarian, 2014). Examples of this travel-based multitasking behavior abound: listening to music while driving, reading or sleeping while riding public transit, and exercising while walking and bicycling. Some activities are more active, requiring "the deliberate use of one's physical and/or mental faculties" (Circella et al., 2012, p. 83), while others are more passive. This distinction is important in the context of mode choice, as travel modes involving the operation of a vehicle (automobile or bicycle) naturally restrict the types of activities that can be (at least safely) conducted while traveling. Indeed, research suggests that train, bus, and car passengers are most commonly reading, writing, resting, and sleeping, while car drivers are more likely to be listening to audio (see Chapter 4). Additionally, many people find benefit in simply escaping from other obligations or using their commutes as a transition or buffer time between home and work roles (Jain \& Lyons, 2008).

Until recently, there were few studies of travel-based multitasking (Kenyon \& Lyons, 2007). Increasing interest has coincided with the rise in internet-connected electronic devices, and many studies have focused on information and communications technology (ICT) use among public transit passengers (e.g., Lyons et al., 2016), although 
multimodal studies are increasing (Berliner et al., 2015). Common approaches to collecting data on travel activities include passive field observations-in which observers surreptitiously record what travelers appear to be doing and for how long-and selfreported activity diaries, where travelers recall what they were doing during a recent trip. Although activity diaries can suffer from recall or response biases, they do allow for the collection of more detailed activities (e.g., texting vs. reading the news on a smartphone) that may be difficult to distinguish via observation alone. For this reason, the study presented in this chapter used the activity-recall questionnaire approach. See Guo et al. (2015) for a recent summary, discussion, and examples of these different travel-based multitasking data collection approaches.

Another way of understanding the benefits of activity participation during travel is to ask travelers about the subjective value, worth, or usefulness of a trip. Overall, about $20-30 \%$ of travelers consider their travel time to be very worthwhile or mostly useful, while only 10-30\% think travel time is mostly wasted (Circella et al., 2015; Lyons et al., 2016; Rosenfield \& Zhao, 2016). See other chapters for more information on the travel activity component of the PUT concept (Chapter 2) and about the measurement of travelbased multitasking and travel usefulness (Chapter 5).

\subsubsection{Travel experiences}

The second and more likely pathway to reaching a PUT is via benefits obtained through the act or experience of traveling. Positive travel experiences could generate positive emotions and/or a greater sense of enjoyment, satisfaction, or fulfillment: traveling out of the way to experience a more scenic route, enjoying views of fall leaves or spring 
flowers, and feeling excited to experience the first snowflake of winter. Some of the motivations for automobile ownership and use are related to feelings of power and control or expressions of social status. For some people who ride the bus or bicycle, their mode choice is in part an expression of their environmental values. Clearly, inherent modal differences can have a large impact on travel experiences, and people may consider these experiences when choosing between different modes for a particular trip. Indeed, research consistently finds that walking and bicycling are rated as more positive than driving an automobile, and public transit is often more negative (see Chapter 5).

Much of the benefits of positive travel experiences can be summarized through their impact on subjective well-being (SWB). Well-being is a broad concept that is closely related to satisfaction, happiness, health, and quality of life. It is a common subject in the field of psychology, and researchers are starting to investigate its connections with transportation (Delbosc, 2012; Nordbakke \& Schwanen, 2014; Reardon \& Abdallah, 2013). SWB is typically divided into two aspects: hedonic SWB - preference satisfaction, mood, pleasure, and happiness - and eudaimonic SWB, finding meaning, purpose, and self-actualization (De Vos et al., 2013). Subsequently, hedonic SWB is often split into affective (positive and negative emotions) and cognitive aspects (Diener, 1984). SWB tends to be measured using self-reported answers to a series of survey questions, and many well-established psychometric instruments exist (Ettema et al., 2010; De Vos et al., 2013; Mokhtarian, in progress). Unfortunately, most SWB scales have not been examined in the context of travel behavior, in part because they are not easily translated to shorter time scales or to the travel domain (see Chapter 5). 
One SWB instrument that has been specifically designed and tested for travel behavior is the Satisfaction with Travel Scale (STS), developed by Ettema and colleagues (Ettema et al., 2011). Based on two-dimensional conceptions of core affect (Russell, 1980; Västfjäll et al., 2002), the STS measures both affective and cognitive components of hedonic SWB using responses to multiple paired statements about feelings and experiences on a recent trip. Multiple studies from northern Europe, North America, and China have validated the structure of the STS, mostly for commuting (see Chapter 5). This study uses a version of the STS designed to be more applicable to an English-language audience.

Another relatively common measure of travel affect involves asking about a general affinity for travel or travel liking (Mokhtarian \& Salomon, 2001; Ory \& Mokhtarian, 2005). Again, commute travel is the most frequently studied travel behavior; only about $30-40 \%$ of people report disliking their home-to-work travel. Several other review and empirical papers offer additional detail on how travel experience aspects of the PUT conceptincluding the STS and travel liking - are conceived, measured, and modeled (Chapter 2, Chapter 5).

\subsubsection{PUT and mode choice}

Despite recent attention to PUT-related aspects, a limited number of studies have examined any effects of travel activities and travel experiences on traveler behaviors (see Chapter 2). Fewer still have focused on the roles of travel-based multitasking or subjective well-being with respect to the mode choice decision.

Within mode choice studies of PUT, travel-based multitasking has been more frequently analyzed than SWB. In two stated preference studies based in the Netherlands, 
multitasking-related amenities of train travel over car travel (sitting down, table space, internet access, and quiet compartments) did not impact stated mode choice (van der Waerden et al., 2010), but commuters with an inclination to multitask and who listened to music had a lower sensitivity to travel time (Ettema \& Verschuren, 2007). A revealed preference study of commuters in Northern California (Malokin et al., 2015) found a positive association between the perceived multitaskability of a given mode and the utility of that mode. A propensity to use a laptop or tablet while commuting was also found to significantly increase the utility of using commuter rail and carpooling.

Few studies have investigated the potential role of expectations of positive emotions or increased SWB on mode choice. Studies of attitudes and noninstrumental motivations for car use suggest that positive perceptions or enjoyment of driving could make people more likely to drive (Gardner \& Abraham, 2008; Zhao \& Zhao, 2015). In fact, a stated preference study found that people who placed a greater importance on gaining happiness from travel were more likely to drive than to ride public transit (Duarte et al., 2010).

One reason why so few PUT studies analyze mode choices (and why mode choice studies almost never include measures of the PUT concept) is that gathering the requisite data can be challenging and burdensome. PUT attributes can be measured at different scales: They can be about travel in general ("I like traveling" or "Making use of my travel time is very important to me"); they can be mode-specific, referring to general assessments of travel by different modes ("I like bicycling" or "It's easy to multitask on public transit"); or they can be trip-specific, referring to experiences on a particular trip or a purpose-mode combination ("That was a fun walk" or "I usually spend most of my bus commute 
reading"). The more general measures could be considered to be attitudes or other more stable characteristics of travelers (e.g. values, personality traits) that vary between decisionmakers but not over alternatives. A standard travel survey questionnaire could collect these data once alongside socio-demographic characteristics. On the other hand, all of the trip-specific and most of the mode-specific metrics are more akin to perceptions that vary across alternatives ("I don't like driving" or "I wouldn't be able to read if I drove"). Thus, this relevant information must be available for all modes considered. BahamondeBirke and colleagues (Bahamonde-Birke, Kunert, Link, \& Ortúzar, 2017) go into more detail about how to conceptualize, distinguish, and treat attitudes and perceptions in choice models. To summarize, PUT-related measures of travel activities and travel experiences are more like perceptions than attitudes, and they must be treated as such in mode choice models by collecting or constructing them for all choice alternatives.

Previous mode choice research on PUT (described above) has almost never collected these measures for all modal alternatives. Instead, one of two approaches are often employed. First, by using stated preference methods, some studies can have complete attributes of alternatives that are assigned by the analyst by design (Duarte et al., 2010; Ettema \& Verschuren, 2007; van der Waerden et al., 2010). However, this approach can assess only a limited number of attributes that vary across alternatives (thus a limited depiction of the PUT concept), and it suffers from potential biases due to hypothetical choice scenarios (Hensher, 2010). Second, revealed preference studies have measured PUT aspects for a single chosen mode, using that information to model the same attributes for nonchosen alternatives (Malokin et al., 2015). These unobserved PUT attributes (e.g., the propensity to multitask) have been modeled as function of other trip and traveler 
characteristics in an approach somewhat like the use of instrumental variables. Unfortunately, this method requires that the propensity models explain a large portion of the observed variance - a requirement unlikely to be met in practice-and makes the restrictive assumption that nonusers with similar characteristics would have the same multitasking behavior or emotional experiences as users of a particular mode.

This study attempts to address these major gaps in knowledge by examining how both aspects of the PUT concept — travel activities and travel experiences - influence mode choice behaviors. In particular, it makes a contribution by explicitly measuring these modespecific PUT attributes for all alternatives, thus avoiding the troublesome approaches described above.

\subsection{Data and methods}

\subsubsection{Data collection}

The analyses presented in this chapter rely on a 30-minute online questionnaire survey administered to working and commuting adults in the Portland, Oregon, region. Respondents were asked to report detailed information about their most recent commute trip from home to work, including information on travel-based multitasking, travel usefulness, travel affect, travel eudaimonia, the satisfaction with travel scale, and travel liking. Data were collected between mid-October and mid-December 2016, and participants were primarily recruited via email at their place of employment. Although 791 people started the survey, only 576 people completed enough questions to be used in these analyses. For more information on the data collection process, see Chapter 3. Descriptive 
statistics of the sample and the independent variables used in the mode choice model are shown in Table 4.2.

One of the most important tasks for any discrete choice analysis is the construction of realistic consideration choice sets. In this study, unlike in many others, choice sets of alternatives were solicited and reported by respondents as part of the data collection effort. In addition to their chosen mode, respondents were instructed to "select at least one other mode, but select all that you considered using." As a result, choice sets were atypically sparse: $70 \%$ of cases had only two modal alternatives, $27 \%$ had three alternatives, and less than $3 \%$ had four or five modes from which to choose. However, these choice sets were likely more realistic than those from datasets relying on analyst-specified construction rules, yielding more behaviorally relevant parameter estimates.

In the estimation dataset, 576 mode choices with complete information were observed. Driving an automobile was available for $76 \%$ of respondents, and public transit was an available mode for $68 \%$ of cases. About $37 \%$ of people considered bicycling and riding as a passenger in an automobile. Walking was an available mode for less than $14 \%$ of commuters. Almost $62 \%$ of commuters chose driving when available. Bicycling, riding public transit, and walking were chosen $47 \%, 39 \%$, and $30 \%$ of the time, respectively. Only $14 \%$ of travelers chose to ride as an automobile passenger when presented with the option. Several types of variables were analyzed in the mode choice model. Traveler characteristics collected as part of the survey included demographic and socioeconomic attributes, as well as perceptions about travel time related to the PUT concept. Before these independent variables entered the model, they were examined for multicollinearity issues; variables that were moderately-to-strongly correlated (>0.40) were removed. To this were 
added information about weather-temperature, precipitation—on the travel day. Weather data were obtained from the Local Climatological Data product of the National Centers for Environmental Information (NOAA, 2017). Each commute trip was associated with weather information on the travel day for the fixed station (usually located at an airport) closest to home but no more than 20 miles away.

Level-of-service information — travel time and cost—were also collected for each alternative in the choice set. Although respondents provided this information for chosen modes, they did not do so for alternative modes. Furthermore, self-reported travel time is known to be susceptible to significant rounding, perception, and memory biases (Rietveld, 2002; Witlox, 2007), and reported costs were inconsistent (despite detailed instructions). Therefore, travel times and costs were calculated for all modal alternatives in a standardized way. Mode-specific travel times were gathered using Google Maps Directions API (Google, 2017c), queried through the googleway package (Cooley, 2016) in R. This procedure obtained Google-estimated shortest travel times between specific geocoded home and work locations (or nearby intersections) for average traffic conditions on the specific day of the week and at the observed departure time from home. Thus, travel times took into account typical roadway traffic congestion (for automobile modes) and transit service schedules (for public transit). Google's algorithms assumed average walking speeds of around three mph and average bicycling speeds of around $10 \mathrm{mph}$.

Some travel time adjustments were made to create more realistic and comparable door-to-door travel times. The Directions API for transit considered only walking access, so separate "park-and-ride" travel times were calculated by querying driving trips from home to the nearest transit station with at least 150 parking spaces and transit-plus-walking 
trips from that transit station to work. For travelers owning automobiles, if the park-andride transit trip was at least $20 \%$ or 10 minutes faster than the walk access transit trip, the park-and-ride transit travel time was used instead. Because Google Maps Directions travel times did not include any initial wait time (resulting from mismatches between desired and scheduled departure times), five minutes were added to all transit trips; an additional one minute of vehicle start-up and two minutes of parking time were added to every park-andride transit trip. The same one-minute vehicle start-up time was added to all bicycle and automobile trips as well. Two minutes for end-of-trip parking time were also added for automobile drivers; only one minute of drop-off time was added for vehicle passengers. Since parking in Downtown Portland can be more difficult and time consuming, and because parking lots and garages may be further from workplaces, additional parking time was added to these trips. Automobile trips ending in Portland's Central City received two additional minutes of parking time, while those ending in the heart of the Central Business District also received an additional three minutes of parking/walking time. Automobile passengers received a 50\% discount on parking/walking time, as it was assumed that they might be dropped off. Finally, all travel times less than five minutes were rounded up to this minimum value. Altogether, these manual edits generated travel times that more closely matched self-reported times for all modes than the original Google-queried travel times.

Travel costs were constructed in a manner similar to that used in a previous mode choice study in Portland (Singleton \& Wang, 2014). Costs for walking and bicycling were zero. Transit costs were provided by the Google Maps Directions API; most transit trips were $\$ 2.50$, the standard 2.5-hour adult fare for TriMet, the regional transit agency. Fares 
for travelers reporting an employer-provided transit pass were reduced to $\$ 1.00$ to reflect an average subsidy. Automobile costs were more complicated to construct. First, fuel costs were calculated using home-to-work travel distances (obtained from the Directions API), an average gas price of $\$ 2.50$ in the Portland region during fall 2016 (GasBuddy, 2017), and an average light-duty vehicle fleet fuel efficiency of $20 \mathrm{mpg}$ (BTS, 2017), yielding a $\$ 0.125$ cost per mile. For trips to the Portland Central Business District, a $\$ 5.00$ per-trip parking fee was added; monthly and daily garage rates were roughly $\$ 10-12$ per day (PBOT, 2017). For trips outside the CBD but within the Central City or within two blocks (0.10 miles) of a parking meter, a $\$ 4.00$ per-trip parking cost was added, assuming parking for four hours at $\$ 1.00$ per hour. Automobile costs for passengers were halved, assuming costs were shared equally with drivers. After all of these changes, travel times and travel costs were not highly correlated -0.53 for automobile drivers, 0.45 for automobile passengers, and 0.17 for public transit passengers-suggesting that multicollinearity problems would be unlikely.

One novel aspect of this dataset is the measurement of PUT-related attributes not just for a single chosen mode but also for the modal alternatives in a commuter's consideration choice set. Previous research integrating PUT concepts into mode choice models have not done this. As described earlier in the literature review, most approaches either use stated preference methods and assign a limited number of PUT attributes to alternatives (e.g., van der Waerden et al., 2010), or they model PUT propensities as a function of other nonvarying characteristics (e.g., Malokin et al., 2015). The modeling approach may not provide valid results, especially if the propensity models do not have a strong goodness-of-fit; $R^{2}$ values in previous research with this dataset (Chapter 4, Chapter 
5) rarely exceeded 0.50 . To overcome these obstacles, this study simply asked commuters the same questions about travel activity and travel experience aspects of the PUT concept for at least one alternative mode. Question wordings and response scales remained the same. Of course, this approach did not negate some unrealistic response issues. For instance, commuters were likely more familiar with their chosen mode than with alternative modes, so some responses for these nonchosen modes may have been based on limited experience. Additionally, confirmation bias or cognitive dissonance (Festinger, 1957; Nederhof, 1985) may also have played a role.

Travel activity aspects were primarily measured by responses to questions about travel-based multitasking. Respondents were asked first to select which of 23 distinct activities they conducted while commuting, and next to provide an approximate percentage of the travel time spent doing each activity. This information, along with the constructed mode-specific travel times, was used to calculate two measures of travel-based multitasking: activity participation (binary) and activity duration (minutes). Exploratory factor analysis grouped six activities into two categories: information and communication technology or "ICT"-related activities, and "passive" activities. After removing those with low response frequencies, 15 activities or activity groups were retained. A single-item question of travel usefulness - measured on a five-point Likert-type scale, Very wasted ... Very useful — was also included in the mode choice models. For more information on these travel activity measures of PUT, see Chapter 4.

The travel experience aspects were measured by the Satisfaction with Travel Scale (STS). The STS captures two primary aspects of hedonic SWB resulting from travel: affective or emotional aspects (positive and negative feelings), and an overall cognitive 
evaluation. For each of nine paired statements, respondents selected a choice on a sevenpoint semantic differential scale that best corresponded to their overall experience. These responses were then structured using exploratory and confirmatory factor analysis (see Figure 5.1), yielding the expected three unobserved constructs "Positive deactivation" (PD), "Positive activation" (PA), and "Cognitive evaluation" (CE), as well as the overall STS concept "Commute satisfaction." For more information on the STS and its construction, see Chapter 5. A single-item question about travel liking-measured on a five-point Likert-type scale, Strongly disliked ... Strongly liked —was also included in the mode choice models, since it has been used in previous studies (Mokhtarian \& Salomon, 2001; Ory \& Mokhtarian, 2005; Turcotte, 2006).

\subsubsection{Analysis methods}

Although the mode choice decision process is typically modeled using discrete choice analysis (DCA) methods, these techniques are not sufficient to include the unobserved or latent variables (LVs) representing travel experience PUT attributes. Notably, the STS and its LVs were constructed using confirmatory factor analysis (CFA), a subset of structural equation modeling (SEM). A technique to link SEM and DCA that is growing more popular is integrated (discrete) choice and latent variable (ICLV) modeling, sometimes called hybrid choice modeling. ICLV modeling allows for the benefits of SEM — testing structural relationships and theories, accounting for measurement error-to be utilized within a DCA framework (Vij \& Walker, 2016). The statistical methodology for specifying and estimating ICLV models was developed by a number of authors during the 1980s and 1990s (Ben-Akiva et al., 1999; Ben-Akiva et al., 2002; Morikawa, Ben- 
Akiva, \& McFadden, 2002; Walker, 2001) but did not see rapid growth until recent increases in computational power; advances in estimation continue today (Bhat \& Dubey, 2014). In short, ICLV models allow for unobserved LVs that are measured by observed indicators in an SEM to directly enter the utility equations of a DCA model.

What unobserved variables would analysts want to include in a model of mode choice? Most studies seek to account for the roles of attitudes, perceptions, values, and other psychosocial attributes of travelers or alternatives. Bahamonde-Birke et al. (2017) offer valuable distinctions between these concepts and their treatment in ICLV models. The most common type of latent variable analyzed is an attitude not related to any alternative, which is assumed to vary across individuals but be stable (at least at one point in time) across alternatives. This attribute could be an environmental attitude, a concern for safety, or a polychronic (proclivity to multitask) personality trait. Most ICLV implementations (e.g., Abou-Zeid \& Ben-Akiva, 2011) utilize this type of LV, and it can be treated as any other socio-demographic traveler characteristic: either as an adjustment to the alternative-specific constants, or by interacting it with alternative-varying variables (like travel time) to examine taste variation (Bahamonde-Birke et al., 2017).

The second group of latent variables are attitudes related to alternatives, such as a liking of bicycle travel in general. While these attributes are characteristics of an individual that do not vary across alternatives (presumably, someone still likes bicycling even when walking), they are by definition related to a specific mode and could be included for that choice alternative alone. The final type of LVs are individual-specific perceptions that also vary across alternatives. Examples include travelers' perceptions of the comfort, safety, or anticipated enjoyment of each alternative in a specific mode choice decision context. 
Because these attributes - like travel time and cost—vary across alternatives, they can be specified to have either generic or alternative-specific coefficients.

There is a stronger case to be made for using latent perceptions in mode choice models than for using latent attitudes, not only because perceptions may be more closely related to choices than observed attributes of alternatives (Singleton, 2013), but also because attitudinal models have been criticized for their lack of policy relevance given that attitudes are, by definition, fundamentally static characteristics of individuals that may not be easily influenced by interventions (Chorus \& Kroesen, 2014). (Of course, the word "attitude" has historically been used rather loosely in the travel behavior field, and there are important research needs regarding the formation and adjustment of attitudes in response to various changes in situation, environment, or behavior.) Unfortunately, very few ICLV studies actually incorporate true perceptions, for reasons made clear by Bahamonde-Birke et al. (2017): "it is necessary to gather a new set of [perceptual] indicators for every alternative...that the individual faces...[leading] to a significant increase in the information collected..." (p. 478).

As noted in the description of data above, this study specifically addressed this issue by directly capturing perceptions of the travel experience — as indicated by responses to the STS items - for every choice alternative faced by the respondent. A general ICLV model with latent alternative-specific perceptions can be specified according to the following set of equations:

$$
\begin{gathered}
\text { Discrete choice component: } \\
y_{j n}=\left\{1 \text { if } U_{j n} \geq U_{j^{\prime} n} \text { for } j^{\prime} \in\{1, \ldots, \mathrm{J}\}, 0 \text { otherwise }\right\} \\
U_{j n}=\mathbf{B} \mathbf{1}_{\mathbf{j}} \mathbf{X}_{\mathbf{j n}}+\mathbf{B} \mathbf{2}_{\mathbf{j}} \boldsymbol{\eta}_{\mathbf{j n}}+\varepsilon_{j n} \quad \text { or } \quad U_{j n}=A S C_{j}+\sum_{k} B 1_{k j} \cdot X_{k j n}+\sum_{s} B 2_{s j} \cdot \eta_{s j n}+\varepsilon_{j n}
\end{gathered}
$$


Structural component:

$$
\boldsymbol{\eta}_{\mathbf{j n}}=\boldsymbol{\Gamma}_{\mathbf{j}} \mathbf{X}_{\mathbf{j n}}+\zeta_{\mathbf{j n}} \quad \text { or } \quad \eta_{s j n}=\alpha_{s j n}+\sum_{k} \gamma_{k j n} \cdot X_{k j n}+\zeta_{s j n}
$$

Measurement component:

$$
\mathbf{i}_{\mathbf{j n}}=\Lambda_{\mathbf{j}} \boldsymbol{\eta}_{\mathbf{j n}}+\xi_{\mathbf{j n}} \quad \text { or } \quad i_{m j n}=v_{m j n}+\sum_{s} \lambda_{s m j} \cdot \eta_{s j n}+\xi_{m j n}
$$

where the indices $\mathrm{j}, \mathrm{n}, \mathrm{k}, \mathrm{s}$, and $\mathrm{m}$ refer to alternatives, individuals, exogenous variables $\mathbf{X}$, $S$ latent variables $\boldsymbol{\eta}$, and $M$ indicator variables $\mathbf{i}$, respectively. Note that the latent variables $\boldsymbol{\eta}$ predict both the measurement indicators $\mathbf{i}$ and the utilities $U_{j n}$ of choice alternatives. The stochastic elements $\varepsilon_{j n}, \zeta_{s j n}$, and $\xi_{m j n}$ are assumed to be mutually independent. Assuming the utility error terms $\varepsilon_{j n}$ are independently and identically distributed (IID) Gumbel (GEV Type I) with location zero and scale one yields the familiar multinomial logit kernel for the discrete choice component. The latent variable errors $\zeta_{\text {sjn }}$ are typically assumed to be normally distributed with zero mean and a covariance matrix $\boldsymbol{\Psi}$. Assuming continuous indicators (which is reasonable for the STS, given its 7-point scale), the measurement equation errors $\xi_{m j n}$ are also assumed to be normally distributed with zero mean and covariance matrix $\Theta$.

Following Vij and Walker (2016) and others, and based on the above distributional assumptions, parameters $\mathbf{B} \mathbf{1}_{\mathbf{j}}, \mathbf{B} \mathbf{2}_{\mathbf{j}}, \boldsymbol{\Gamma}_{\mathbf{j}}$, and $\boldsymbol{\Lambda}_{\mathbf{j}}$ can be estimated by maximizing the (log) likelihood function, represented by the following joint unconditional probability distribution function for a single observation:

Likelihood function:

$$
\begin{gathered}
f_{\mathbf{y}, \mathbf{i}}\left(y_{j n}, \mathbf{i}_{\mathbf{j} \mathbf{n}} \mid \mathbf{X}_{\mathbf{j n}} ; \mathbf{B} \mathbf{1}_{\mathbf{j}}, \mathbf{B} \mathbf{2}_{\mathbf{j}}, \boldsymbol{\Lambda}_{\mathbf{j}}, \boldsymbol{\Theta}, \boldsymbol{\Gamma}_{\mathbf{j}}, \boldsymbol{\Psi}\right)= \\
\int_{\mathfrak{\eta}} f_{\mathbf{y}}\left(y_{j n} \mid \mathbf{X}_{\mathbf{j n}}, \boldsymbol{\eta}_{\mathbf{j n}} ; \mathbf{B} \mathbf{1}_{\mathbf{j}}, \mathbf{B} \mathbf{2}_{\mathbf{j}}\right) \cdot f_{\mathbf{i}}\left(\mathbf{i}_{\mathbf{j} \mathbf{j}} \mid \mathbf{X}_{\mathbf{j n}}, \boldsymbol{\eta}_{\mathbf{j n}} ; \boldsymbol{\Lambda}_{\mathbf{j}}, \boldsymbol{\Theta}\right) \cdot f_{\mathbf{\eta}}\left(\boldsymbol{\eta}_{\mathbf{j n}} \mid \mathbf{X}_{\mathbf{j n}} ; \boldsymbol{\Gamma}_{\mathbf{j}}, \boldsymbol{\Psi}\right) \mathrm{d} \boldsymbol{\eta}_{\mathbf{j n}}
\end{gathered}
$$


Discrete choice component:

$$
\begin{aligned}
& f_{\mathbf{y}}\left(y_{j n} \mid \mathbf{X}_{\mathbf{j n}}, \boldsymbol{\eta}_{\mathbf{j n}} ; \mathbf{B} \mathbf{1}_{\mathbf{j}}, \mathbf{B} \mathbf{2}_{\mathbf{j}}\right)=\prod_{j}\left[P\left(y_{j n}=1 \mid \mathbf{X}_{\mathbf{j n}}, \boldsymbol{\eta}_{\mathbf{j n}} ; \mathbf{B} \mathbf{1}_{\mathbf{j}}, \mathbf{B} \mathbf{2}_{\mathbf{j}}\right)\right]^{\mathbf{y}} \\
& P\left(y_{j n}=1 \mid \mathbf{X}_{\mathbf{j n}}, \boldsymbol{\eta}_{\mathbf{j n}} ; \mathbf{B} \mathbf{1}_{\mathbf{j}}, \mathbf{B} \mathbf{2}_{\mathbf{j}}\right)=\exp \left(\mathbf{B} \mathbf{1}_{\mathbf{j}} \mathbf{X}_{\mathbf{j n}}+\mathbf{B} \mathbf{2}_{\mathbf{j}} \boldsymbol{\eta}_{\mathbf{j n}}\right) \div \Sigma_{j^{\prime}} \exp \left(\mathbf{B} \mathbf{1}_{\mathbf{j}} \mathbf{X}_{\mathbf{j}}{ }^{\prime} \mathbf{n}+\mathbf{B} \mathbf{2}_{\mathbf{j}} \boldsymbol{\eta}_{\mathbf{j}} \mathbf{j}_{\mathbf{n}}\right) \\
& \text { Measurement component: } \\
& f_{\mathbf{i}}\left(\mathbf{i}_{\mathbf{j} \mathbf{n}} \mid \mathbf{X}_{\mathbf{j n}}, \boldsymbol{\eta}_{\mathbf{j n}} ; \boldsymbol{\Lambda}_{\mathbf{j}}, \boldsymbol{\Theta}\right)=(2 \pi)^{-0.5 M}|\boldsymbol{\Theta}|^{-0.5} \exp \left[-0.5\left(\mathbf{i}_{\mathbf{j n}}-\boldsymbol{\Lambda}_{\mathbf{j}} \boldsymbol{\eta}_{\mathbf{j n}}\right)^{\mathrm{T}} \boldsymbol{\Theta}^{-1}\left(\mathbf{i}_{\mathbf{j n}}-\boldsymbol{\Lambda}_{\mathbf{j}} \boldsymbol{\eta}_{\mathbf{j n}}\right)\right] \\
& \text { Structural component: } \\
& f_{\mathbf{\eta}}\left(\boldsymbol{\eta}_{\mathbf{j n}} \mid \mathbf{X}_{\mathbf{j n}} ; \boldsymbol{\Gamma}_{\mathbf{j}}, \boldsymbol{\Psi}\right)=(2 \pi)^{-0.5 s}|\boldsymbol{\Psi}|^{-0.5} \exp \left[-0.5\left(\boldsymbol{\eta}_{\mathbf{j n}}-\boldsymbol{\Gamma}_{\mathbf{j}} \mathbf{X}_{\mathbf{j n}}\right)^{\mathrm{T}} \boldsymbol{\Psi}^{-1}\left(\boldsymbol{\eta}_{\mathbf{j n}}-\boldsymbol{\Gamma}_{\mathbf{j}} \mathbf{X}_{\mathbf{j n}}\right)\right]
\end{aligned}
$$

where the first term reflects the discrete choice component, the second term reflects the measurement component, and the third term reflects the structural component.

Mode choice models were specified as follows. Traveler characteristicsdemographic and socioeconomic attributes, travel time perceptions, and weather—did not vary across alternatives $\left(X a_{k j n}=X a_{k n}\right.$ for $\left.j \in\{1, \ldots, J\}\right)$ and so were specified to have alternative-specific coefficients $\left(B l a_{k j}\right)$ relative to a reference alternative (driving). Levelof-service attributes - travel time and cost-varied across alternatives $\left(X b_{k j n}\right)$ and were specified to have generic coefficients $\left(B 1 b_{k j}=B 1 b_{k}\right.$ for $\left.j \in\{1, \ldots, J\}\right)$ that were equal across modal alternatives. A mode $\times$ travel time interaction was examined (model not shown) and generated a significant improvement in model fit $(p<0.05)$, but alternative-specific travel time coefficients were not significant or had unintuitive positive signs. Travel activity characteristics - activity participation, activity duration, and travel usefulness-were specified with generic coefficients $\left(B l_{k} j=B 1 c_{k}\right.$ for $\left.j \in\{1, \ldots, \mathrm{J}\}\right)$. Model testing indicated no significant difference in travel usefulness across modes. Models interacting mode with activity participation or duration had empirical identification problems with zero cells; several activities were rarely or never reported for some modes. Travel experience 
characteristics - the STS and travel liking — were also specified with generic coefficients. Again, model testing indicated no significant difference in travel liking across modes. Models interacting mode with the STS did improve goodness-of-fit $(p<0.05)$, but modespecific STS coefficients had roughly similar magnitudes; therefore, generic coefficients $\left(B 2_{s j}=B 2_{s}\right.$ for $\left.j \in\{1, \ldots, J\}\right)$ were used for interpretability. For simplicity, no explanatory variables were included in the structural models predicting the latent variables $\left(\boldsymbol{\Gamma}_{\mathbf{j}}=\mathbf{0}\right)$.

ICLV models were estimated simultaneously using Python Biogeme Version 2.3 (Bierlaire, 2016), using maximum simulated likelihood estimation with CFSQP nonlinear optimization (Lawrence, Zhao, \& Tits, 1994) and 1,000 random draws according to a Modified Latin Hypercube Sampling strategy (Hess, Train, \& Polak, 2006). Given that there were four latent variables of the STS (PD, PA, CE, and overall commute satisfaction) for each of the five modes (walking, bicycling, auto driver, auto passenger, public transit), the models utilized a total of 20 different latent variables.

\subsection{Results}

Several ICLV models were estimated to examine different model specifications and to test the significance of different blocks of variables using nested model comparison tests. In the block of nonvarying trip and traveler characteristics (socio-demographics, perceptions, and weather), variables that were not significant $(p>0.10)$ were excluded from future models for parsimony. For each combination of variables, two ICLV models were estimated: one using activity participation variables $(N=576)$ and one using activity duration variables $(N=546)$. Approximate goodness-of-fit statistics and likelihood ratio model comparison tests for all models are shown in Table 6.1 below. (These summary 
statistics are approximate because only total log-likelihood values were produced by Biogeme, not the log-likelihood portions attributable to the DCA components. Instead, it was assumed that the log-likelihood for the SEM portion remained the same in each model run, which is a reasonable assumption given that the SEM parameter estimates remained relatively stable across different specifications of the utility equations. Thus, any differences in total log-likelihood between model runs were assumed to be attributable to changes in the DCA portion.)

A summary of the goodness-of-fit results will focus on the DCA portion of the ICLV model, as mode choice is the behavior under consideration that we wish to explain. Including only alternative-specific constants reduced almost $12 \%$ of the null model deviance. Adding the two level-of-service (LOS) variables — travel time and cost-with generic coefficients explained about another $11 \%$. Further addition of the nonvarying trip and traveler (T\&T) characteristics variables with alternative-specific coefficients yielded McFadden's $R^{2}$ values approaching 0.47 . Alone, the travel activities variables had pseudo$R^{2}$ values of between 0.21 and 0.26 ; adding travel activities variables to the LOS and T\&T variables approximately doubled the proportion of reduced deviance to around 0.55 . In the activity participation model, the travel experience variables were more explanatory of mode choice than the travel activity variables; however, in the activity duration model, the travel experience variables were actually less explanatory. The complete ICLV modelswith LOS, T\&T, and both sets of PUT variables-had relatively large goodness-of-fit statistics, ranging from a pseudo- $\mathrm{R}^{2}$ of 0.59 for the model with activity duration to 0.66 for the model with activity participation. All nested model comparison likelihood ratio tests were statistically significant. 
More complete results from three final models are presented below. First, results from a basic multinomial logit discrete choice model of mode choice including traditional LOS and T\&T variables (but not PUT measures) is shown in Table 6.2. Next, the estimation results from two ICLV models adding travel activity variables-travel usefulness, plus activity participation or activity duration, as appropriate - and travel experience variables (the STS and travel liking) are presented in Table 6.3 and Table 6.4. (The results for the SEM structural and measurement model components are not shown, although they looked roughly similar to the CFA results of Figure 5.1.) The two ICLV tables present ranges for $\log$-likelihood and $R^{2}$ values for the same reason as described above: Biogeme did not produce component-specific estimates, just an overall value. The range of values reflects different assumptions: either that the SEM log-likelihood values from the models in Table 6.3 and Table 6.4 were the same as from models estimated using the specification of Table 6.2 (like in Table 6.1); or that the DCA log-likelihood values in the full ICLV models were the same as from sequential models estimated in R using predicted STS factor scores. The true log-likelihood values for the DCA portion likely fell somewhere between these two values. 
Table 6.1 Goodness-of-fit statistics for ICLV models of mode choice

\begin{tabular}{|c|c|c|c|c|c|c|c|c|c|c|}
\hline \multirow[b]{3}{*}{ DCA model specification } & \multicolumn{6}{|c|}{ With activity participation $(N=576)$} & \multicolumn{4}{|c|}{ With activity duration $(N=546)$} \\
\hline & \multicolumn{2}{|c|}{ \# param. } & \multicolumn{3}{|c|}{ log-likelihood } & $R^{2}$ & \multicolumn{3}{|c|}{ log-likelihood } & $R^{2}$ \\
\hline & $D C A S$ & $E M$ & $D C A$ & SEM & Total & $D C A$ & $D C A$ & $S E M$ & Total & $D C A$ \\
\hline Null & 0 & 30 & -473.53 & $-17,612$ & $-18,085$ & 0.000 & -449.49 & $-16,675$ & $-17,125$ & 0.000 \\
\hline Constants-only & 4 & 30 & -417.70 & $-17,612$ & $-18,029$ & 0.118 & -396.91 & $-16,675$ & $-17,072$ & 0.117 \\
\hline Level-of-service (LOS) attributes & 6 & 30 & -365.97 & $-17,612$ & $-17,978$ & 0.227 & -349.07 & $-16,675$ & $-17,024$ & 0.223 \\
\hline Trip \& traveler $(\mathrm{T} \& \mathrm{~T})$ characteristics & 80 & 30 & -286.96 & $-17,612$ & $-17,899$ & 0.394 & -266.74 & $-16,675$ & $-16,942$ & 0.407 \\
\hline Travel activities (TA) & 22 & 30 & -373.06 & $-17,612$ & $-17,985$ & 0.212 & -333.05 & $-16,675$ & $-17,008$ & 0.259 \\
\hline Travel experiences (TE) & 8 & 30 & -335.72 & $-17,612$ & $-17,947$ & 0.291 & -355.93 & $-16,675$ & $-17,031$ & 0.208 \\
\hline $\mathrm{LOS}+\mathrm{T} \& \mathrm{~T}$ & 82 & 30 & -254.88 & $-17,612$ & $-17,867$ & 0.462 & -238.22 & $-16,675$ & $-16,913$ & 0.470 \\
\hline $\mathrm{LOS}+\mathrm{T} \& \mathrm{~T}+\mathrm{TA}$ & 100 & 30 & -199.95 & $-17,612$ & $-17,812$ & 0.578 & -215.33 & $-16,675$ & $-16,891$ & 0.521 \\
\hline $\mathrm{LOS}+\mathrm{T} \& \mathrm{~T}+\mathrm{TE}$ & 86 & 30 & -195.28 & $-17,612$ & $-17,807$ & 0.588 & -231.73 & $-16,675$ & $-16,907$ & 0.484 \\
\hline $\mathrm{LOS}+\mathrm{TA}+\mathrm{TE}$ & 26 & 30 & -300.70 & $-17,612$ & $-17,912$ & 0.365 & -304.78 & $-16,675$ & $-16,980$ & 0.322 \\
\hline $\mathrm{LOS}+\mathrm{T} \& \mathrm{~T}+\mathrm{TA}+\mathrm{TE}$ & 104 & 30 & -162.43 & $-17,612$ & $-17,774$ & 0.657 & -185.77 & $-16,675$ & $-16,861$ & 0.587 \\
\hline
\end{tabular}


Table 6.2 DCA results for model with level-of-service attributes and trip and traveler characteristics

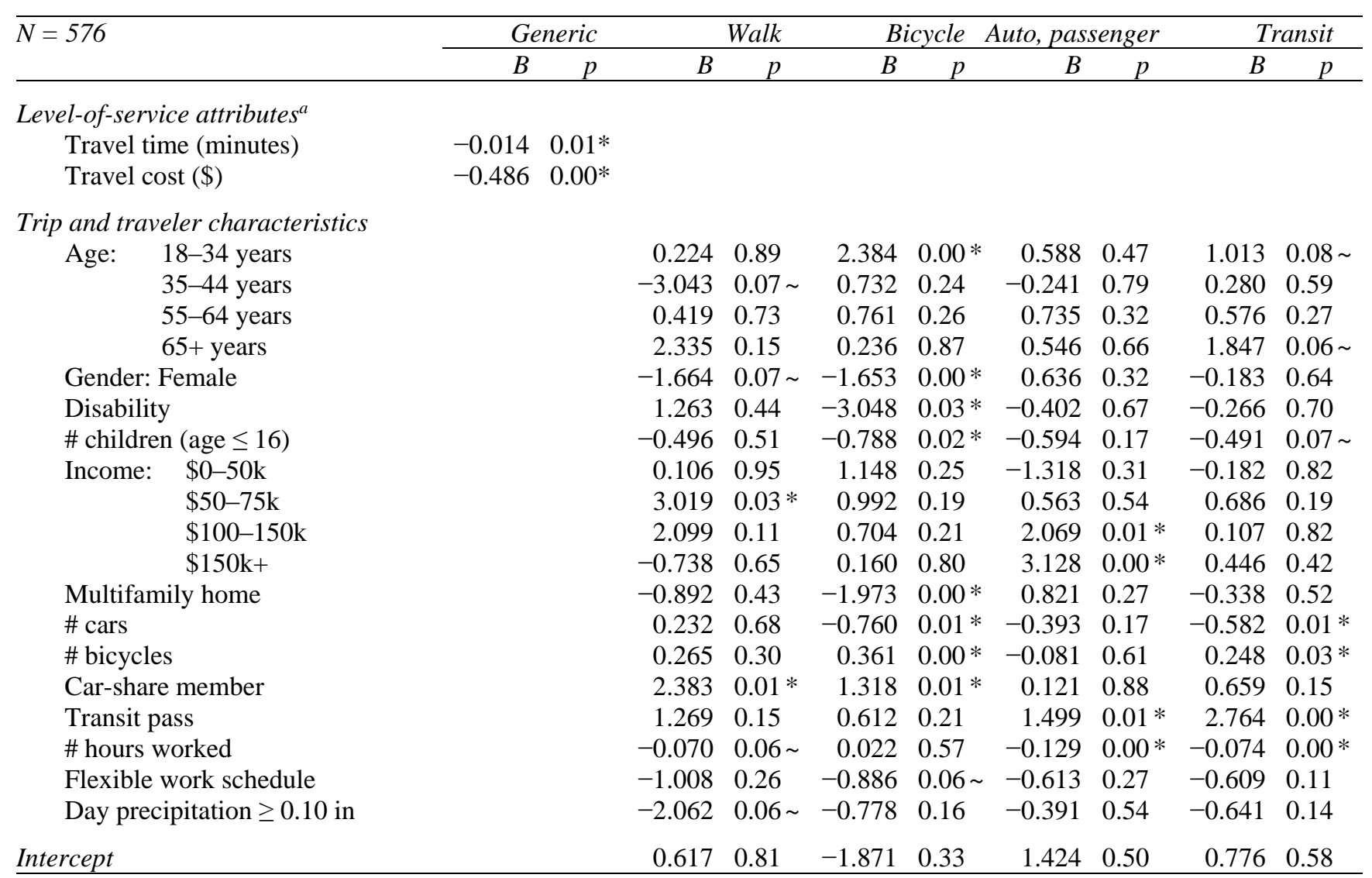

Statistical significance: $*=p \leq 0.05, \sim=p \leq 0.10$.

Baseline mode: Auto, driver.

${ }^{a}$ These variables have generic coefficients that are equal across all five alternatives.

Model fit statistics: $\log$-likelihood $=-254.88, d f=82$, McFadden $R^{2}=0.462$. 
Table 6.3 ICLV results for model with PUT measures of travel activities (participation) and travel experiences

\begin{tabular}{|c|c|c|c|c|c|c|c|c|c|c|}
\hline \multirow[t]{2}{*}{$N=576$} & \multicolumn{2}{|c|}{ Generic } & \multicolumn{2}{|c|}{ Walk } & \multicolumn{2}{|c|}{ Bicycle } & \multicolumn{2}{|c|}{ Auto, passenger } & \multicolumn{2}{|c|}{ Transit } \\
\hline & $B$ & $p$ & $B$ & $p$ & $B$ & $p$ & $B$ & $p$ & $B$ & $p$ \\
\hline
\end{tabular}

Level-of-service attributes

Travel time (minutes)

$-0.0200 .00 *$

Travel cost (\$) -0.691 $0.00 *$

Travel activities ${ }^{a}$

ICT activities

Passive activities

$\begin{array}{lll}\text { Talking with strangers } & -0.422 & 0.24\end{array}$

Talking on the phone $\quad-2.0520 .00 *$

$\begin{array}{lll}\text { Reading print } & -0.837 & 0.07 \sim\end{array}$

Listening to music, radio, audio $\quad 0.094 \quad 0.77$

Playing game $\quad-0.214 \quad 0.66$

Eating; drinking $\quad-0.479 \quad 0.27$

Personal grooming $\quad 2.727 \quad 0.00 *$

Singing; dancing $\quad-0.084 \quad 0.89$

Exercising; physically active $\quad 0.756 \quad 0.21$

Planning or navigating this trip $\quad-1.310 \quad 0.00^{*}$

$\begin{array}{lll}\text { Sleeping or snoozing } & -0.839 & 0.12\end{array}$

Doing nothing $\quad 1.213 \quad 0.08 \sim$

Travel usefulness:

$\begin{array}{lll}\text { Wasted } & 0.705 & 0.07 \sim \\ \text { Somewhat useful } & 0.367 & 0.36 \\ \text { Mostly useful } & 0.825 & 0.09 \sim\end{array}$

Travel experiences ${ }^{a}$

Commute satisfaction (STS) $\quad 1.317 \quad 0.00 *$

Travel liking:

Disliked

$\begin{array}{ll}-0.535 & 0.15\end{array}$

$\begin{array}{lll}\text { Somewhat liked } & 0.138 & 0.70 \\ \text { Strongly liked } & 0.196 & 0.66\end{array}$

N 


\begin{tabular}{|c|c|c|c|c|c|c|c|c|c|c|c|}
\hline \multirow{2}{*}{\multicolumn{2}{|c|}{$\bar{N}=576$}} & \multicolumn{2}{|c|}{ Generic } & \multicolumn{2}{|r|}{ Walk } & \multicolumn{2}{|c|}{ Bicycle } & \multicolumn{2}{|c|}{ Auto, passenger } & \multicolumn{2}{|c|}{ Transit } \\
\hline & & $B$ & $p$ & $B$ & $p$ & $B$ & $p$ & $B$ & $p$ & $B$ & $p$ \\
\hline \multicolumn{12}{|c|}{ Trip and traveler characteristics } \\
\hline \multirow[t]{4}{*}{ Age: } & $18-34$ years & & & -0.449 & 0.81 & 3.158 & $0.00 *$ & 0.352 & 0.72 & 0.839 & 0.30 \\
\hline & $35-44$ years & & & -5.406 & $0.00 *$ & 0.986 & 0.21 & -0.272 & 0.77 & 0.278 & 0.70 \\
\hline & 55-64 years & & & 0.449 & 0.72 & 0.601 & 0.47 & 0.618 & 0.46 & 0.219 & 0.77 \\
\hline & $65+$ years & & & 3.435 & $0.10 \sim$ & -0.486 & 0.80 & 0.364 & 0.78 & 3.076 & $0.04 *$ \\
\hline \multicolumn{2}{|c|}{ Gender: Female } & & & -3.833 & $0.00 *$ & -2.175 & $0.00 *$ & 0.549 & 0.49 & -0.481 & 0.37 \\
\hline \multicolumn{2}{|c|}{ Disability } & & & 1.934 & 0.23 & -2.533 & $0.09 \sim$ & 0.191 & 0.84 & 0.621 & 0.47 \\
\hline \multicolumn{2}{|c|}{$\#$ children $($ age $\leq 16)$} & & & -1.060 & 0.16 & -1.304 & $0.00 *$ & -0.785 & $0.06 \sim$ & -0.868 & $0.01 *$ \\
\hline \multirow[t]{4}{*}{ Income } & $\$ 0-50 \mathrm{k}$ & & & 0.102 & 0.96 & 0.126 & 0.90 & -2.387 & $0.07 \sim$ & -0.108 & 0.90 \\
\hline & $\$ 50-75 \mathrm{k}$ & & & 4.145 & $0.05 *$ & 0.267 & 0.79 & 0.802 & 0.54 & 1.182 & 0.17 \\
\hline & $\$ 100-150 k$ & & & 1.381 & 0.37 & 0.019 & 0.98 & 2.796 & $0.00 *$ & 0.314 & 0.62 \\
\hline & $\$ 150 \mathrm{k}+$ & & & -1.271 & 0.48 & 0.086 & 0.91 & 3.264 & $0.02 *$ & 0.793 & 0.37 \\
\hline \multicolumn{2}{|c|}{ Multifamily home } & & & -0.865 & 0.46 & -2.508 & $0.00 *$ & 1.097 & 0.17 & -0.494 & 0.51 \\
\hline \multicolumn{2}{|c|}{ \# cars } & & & -0.258 & 0.64 & -0.878 & $0.01 *$ & 0.003 & 0.99 & -0.398 & 0.14 \\
\hline \multicolumn{2}{|c|}{ \# bicycles } & & & 0.750 & $0.01 *$ & 0.496 & $0.00 *$ & -0.142 & 0.44 & 0.436 & $0.00 *$ \\
\hline \multicolumn{2}{|c|}{ Car-share member } & & & 2.373 & $0.06 \sim$ & 0.881 & 0.16 & 0.702 & 0.46 & 0.567 & 0.36 \\
\hline \multicolumn{2}{|c|}{ Transit pass } & & & 1.546 & 0.18 & 1.335 & $0.04 *$ & 1.646 & $0.03 *$ & 3.187 & $0.00 *$ \\
\hline \multicolumn{2}{|c|}{ \# hours worked } & & & -0.117 & $0.03 *$ & -0.029 & 0.52 & -0.225 & $0.00 *$ & -0.106 & $0.01 *$ \\
\hline \multicolumn{2}{|c|}{ Flexible work schedule } & & & -2.318 & $0.04 *$ & -1.882 & $0.00 *$ & -0.744 & 0.30 & -1.266 & $0.02 *$ \\
\hline \multicolumn{2}{|c|}{ Day precipitation $\geq 0.10$ in } & & & -2.022 & $0.10 \sim$ & -0.800 & 0.20 & -0.509 & 0.56 & -0.882 & 0.13 \\
\hline \multicolumn{2}{|l|}{ Intercept } & & & 1.892 & 0.48 & -1.151 & 0.60 & 3.630 & 0.17 & 2.160 & 0.30 \\
\hline
\end{tabular}

Statistical significance: $*=p \leq 0.05, \sim=p \leq 0.10$.

Baseline mode: Auto, driver.

${ }^{a}$ These variables have generic coefficients that are equal across all five alternatives.

Model fit statistics: log-likelihood(DCM) $\approx-189.77$ to -162.43, McFadden $R^{2} \approx 0.599$ to 0.657 . 
Table 6.4 ICLV results for model with PUT measures of travel activities (duration) and travel experiences

\begin{tabular}{|c|c|c|c|c|c|c|c|c|c|c|}
\hline \multirow[t]{2}{*}{$N=546$} & \multicolumn{2}{|c|}{ Generic } & \multicolumn{2}{|c|}{ Walk } & \multicolumn{4}{|c|}{ Bicycle Auto, passenger } & \multicolumn{2}{|c|}{ Transit } \\
\hline & $B$ & $p$ & $B$ & $p$ & $B$ & $p$ & $B$ & $p$ & $B$ & $p$ \\
\hline \multicolumn{11}{|l|}{ Level-of-service attributes ${ }^{a}$} \\
\hline Travel time (minutes) & -0.002 & 0.85 & & & & & & & & \\
\hline Travel cost $(\$)$ & -0.706 & $0.00 *$ & & & & & & & & \\
\hline \multicolumn{11}{|l|}{ Travel activities $^{a}$} \\
\hline ICT activities & -0.020 & 0.18 & & & & & & & & \\
\hline Passive activities & -0.029 & $0.00 *$ & & & & & & & & \\
\hline Talking with people you know & 0.029 & 0.22 & & & & & & & & \\
\hline Talking with strangers & -0.134 & $0.08 \sim$ & & & & & & & & \\
\hline Talking on the phone & -0.263 & $0.00 *$ & & & & & & & & \\
\hline Reading print & -0.017 & 0.32 & & & & & & & & \\
\hline Listening to music, radio, audio & 0.001 & 0.89 & & & & & & & & \\
\hline Playing game & 0.017 & 0.71 & & & & & & & & \\
\hline Eating; drinking & 0.002 & 0.96 & & & & & & & & \\
\hline Personal grooming & 0.322 & $0.05^{*}$ & & & & & & & & \\
\hline Singing; dancing & 0.019 & 0.71 & & & & & & & & \\
\hline Exercising; physically active & 0.003 & 0.79 & & & & & & & & \\
\hline Planning or navigating this trip & -0.097 & $0.00 *$ & & & & & & & & \\
\hline Sleeping or snoozing & -0.040 & 0.22 & & & & & & & & \\
\hline Doing nothing & -0.029 & $0.05^{*}$ & & & & & & & & \\
\hline \multicolumn{11}{|l|}{ Travel usefulness: } \\
\hline Wasted & 0.983 & $0.02 *$ & & & & & & & & \\
\hline Somewhat useful & 0.144 & 0.74 & & & & & & & & \\
\hline Mostly useful & 0.461 & 0.40 & & & & & & & & \\
\hline \multicolumn{11}{|l|}{ Travel experiences ${ }^{a}$} \\
\hline Commute satisfaction (STS) & 1.566 & $0.00 *$ & & & & & & & & \\
\hline \multicolumn{11}{|l|}{ Travel liking: } \\
\hline Disliked & -0.801 & $0.05^{*}$ & & & & & & & & \\
\hline Somewhat liked & 0.063 & 0.86 & & & & & & & & \\
\hline Strongly liked & 0.030 & 0.95 & & & & & & & & \\
\hline
\end{tabular}




\begin{tabular}{|c|c|c|c|c|c|c|c|c|c|c|c|}
\hline \multirow{2}{*}{\multicolumn{2}{|c|}{$N=546$}} & \multicolumn{2}{|c|}{ Generic } & \multicolumn{2}{|r|}{ Walk } & \multicolumn{2}{|c|}{ Bicycle } & \multicolumn{2}{|c|}{ Auto, passenger } & \multicolumn{2}{|c|}{ Transit } \\
\hline & & $B$ & $p$ & $B$ & $p$ & $B$ & $p$ & $B$ & $p$ & $B$ & $p$ \\
\hline \multicolumn{12}{|c|}{ Trip and traveler characteristics } \\
\hline \multirow[t]{4}{*}{ Age: } & $18-34$ years & & & -1.325 & 0.42 & 3.365 & $0.00 *$ & 0.000 & 1.00 & 0.769 & 0.37 \\
\hline & $35-44$ years & & & -5.307 & $0.00 *$ & 0.810 & 0.42 & 1.152 & 0.32 & -0.227 & 0.79 \\
\hline & $55-64$ years & & & 0.015 & 0.99 & 0.870 & 0.40 & 1.664 & 0.18 & 0.634 & 0.48 \\
\hline & $65+$ years & & & 2.298 & 0.17 & 0.394 & 0.82 & 0.046 & 0.97 & 2.560 & $0.06 \sim$ \\
\hline \multicolumn{2}{|c|}{ Gender: Female } & & & -2.125 & $0.03 *$ & -1.626 & $0.03 *$ & 0.373 & 0.62 & -0.336 & 0.57 \\
\hline \multicolumn{2}{|c|}{ Disability } & & & 0.433 & 0.80 & -2.697 & $0.05 *$ & -1.098 & 0.33 & 0.582 & 0.55 \\
\hline \multicolumn{2}{|c|}{$\#$ children $($ age $\leq 16)$} & & & -1.506 & $0.06 \sim$ & -0.943 & $0.06 \sim$ & -0.914 & $0.05 *$ & -0.646 & $0.10 \sim$ \\
\hline \multirow[t]{4}{*}{ Income } & $\$ 0-50 \mathrm{k}$ & & & 0.358 & 0.85 & 2.065 & 0.16 & -0.667 & 0.73 & 1.178 & 0.35 \\
\hline & $\$ 50-75 \mathrm{k}$ & & & 2.980 & $0.08 \sim$ & 0.917 & 0.36 & 2.566 & 0.12 & 1.656 & $0.08 \sim$ \\
\hline & $\$ 100-150 k$ & & & 0.363 & 0.79 & 0.127 & 0.88 & 5.180 & $0.00 *$ & 0.432 & 0.54 \\
\hline & $\$ 150 \mathrm{k}+$ & & & -4.440 & $0.03 *$ & -0.380 & 0.65 & 5.951 & $0.00 *$ & 0.667 & 0.46 \\
\hline \multicolumn{2}{|c|}{ Multifamily home } & & & -1.888 & 0.14 & -2.676 & $0.00 *$ & 2.086 & $0.01 *$ & 0.296 & 0.70 \\
\hline \multicolumn{2}{|c|}{ \# cars } & & & -0.160 & 0.76 & -0.882 & $0.03 *$ & -0.708 & $0.04 *$ & -0.330 & 0.29 \\
\hline \multicolumn{2}{|c|}{ \# bicycles } & & & 0.530 & $0.05 *$ & 0.580 & $0.00 *$ & 0.026 & 0.88 & 0.410 & $0.02 *$ \\
\hline \multicolumn{2}{|c|}{ Car-share member } & & & 1.596 & 0.17 & 0.636 & 0.36 & -0.426 & 0.66 & -0.459 & 0.47 \\
\hline \multicolumn{2}{|c|}{ Transit pass } & & & 0.351 & 0.73 & 0.872 & 0.23 & 0.880 & 0.19 & 3.126 & $0.00 *$ \\
\hline \multicolumn{2}{|c|}{ \# hours worked } & & & -0.119 & $0.02 *$ & -0.038 & 0.45 & -0.316 & $0.00 *$ & -0.125 & $0.01 *$ \\
\hline \multicolumn{2}{|c|}{ Flexible work schedule } & & & -2.241 & $0.08 \sim$ & -1.456 & $0.04 *$ & -0.696 & 0.34 & -0.981 & $0.07 \sim$ \\
\hline \multicolumn{2}{|c|}{ Day precipitation $\geq 0.10$ in } & & & -1.002 & 0.37 & -0.975 & 0.18 & 0.531 & 0.48 & -1.060 & 0.13 \\
\hline \multicolumn{2}{|l|}{ Intercept } & & & 5.847 & $0.08 \sim$ & -0.586 & 0.81 & 5.752 & $0.02 *$ & 2.135 & 0.36 \\
\hline
\end{tabular}

Statistical significance: $*=p \leq 0.05, \sim=p \leq 0.10$.

Baseline mode: Auto, driver.

${ }^{a}$ These variables have generic coefficients that are equal across all five alternatives.

Model fit statistics: log-likelihood(DCM) $\approx-185.77$ to -166.84, McFadden $R^{2} \approx 0.587$ to 0.629 . 
Associations between mode choice and nonvarying traveler and trip characteristics were relatively stable between the non-PUT model and both of the ICLV models; the latter model results are interpreted. Walking was positively associated with age: Younger adults were less likely to walk, especially those aged $35-44$. Women were much less likely to walk than were men, as were people who worked more hours each week and who had flexible work schedules. There was a slight negative association between walking and household income, although this was more apparent in the model with activity durations. Other negative factors were (marginally) significant in only one of the two ICLV models: the number of children and precipitation. Bicycle ownership was positively associated with walking; having a car-share membership seemed was a positive factor only in the model with activity durations.

For bicycling, age was a strong negative factor: Younger adults aged 18-34 had nearly 25 times the odds of bicycling over driving than did middle-aged adults. Gender was also significant and negative: Women had between 0.11 and 0.19 times the odds of bicycling as compared to men. Other factors negatively associated with bicycling included reporting a disability, having more children, living in a multifamily home, owning more automobiles, and having a flexible work schedule. People owning more bicycles and holding a transit pass were more likely to select bicycling over driving.

One of the strongest effects for automobile passengers was a positive association with income: People in households making over $\$ 100,000$ per year had at least 15 times higher odds of being a passenger than travelers with middle and lower incomes. In one model, holding a transit pass was a positive factor; in the other, living in a multifamily building was a positive factor while owning more automobiles was a negative factor. In 
both models, people with a greater number of children and who worked more hours per week were less likely to choose to commute as a car passenger.

Associations between socio-demographic characteristics and transit mode choice were consistent between the two ICLV models. In both, older adults (aged 65+) were significantly more likely to commute by transit instead of driving than younger adults. Unsurprisingly, people with transit passes were more likely to ride public transit; owning more bicycles and living in a lower-middle income household were also positive factors. Characteristics negatively associated with transit mode choice included the number of children, number of hours worked, and having a flexible work schedule.

Level-of-service variables were also influential in expected directions. Travel cost had a negative association with mode choice: Every additional dollar decreased the odds of choosing a particular alternative by about $50 \%\left(\mathrm{e}^{\mathrm{B}}=0.49\right.$ to 0.50$)$. In the model with activity participation, travel time was also negative and significant: A 10-minute increase in travel time yielded almost a $20 \%$ reduction in the odds of choosing a particular mode $\left(\mathrm{e}^{10 \mathrm{~B}}=0.82\right)$. The empirically derived value of travel time savings in this model was about $\$ 1.74 /$ hour $\left(\mathrm{VTTS}=60 \cdot \mathrm{B}_{\mathrm{TT}} / \mathrm{B}_{\mathrm{CO}}\right.$ ), which is considerably lower than is typically found in mode choice analyses. This is a slight decrease from the implied VTTS of $\$ 1.78 /$ hour from the model presented in Table 6.2 without measures of the PUT concept, and a substantial decrease from a VTTS of $\$ 2.16 /$ hour in a model with only LOS variables (not shown).

In the model with activity duration, notably, travel time was a negative but not significant factor. The insignificance of the travel time parameter could be a result of including and accounting for time spent doing other activities; in fact, in earlier models 
predicting travel usefulness (Chapter 4), travel time was a significant predictor in models with activity participation but not in models with activity durations. Indeed, assuming that $100 \%$ of travel time was spent doing nothing yields an increased implied value of travel time savings of $\$ 2.62 /$ hour $\left(\mathrm{VTTS}=60 \cdot\left(\mathrm{BTT}+\mathrm{BDN}_{\mathrm{DN}}\right) / \mathrm{BCO}\right)$. Another likely explanation for both the insignificance of travel time and for the relatively low subjective value of travel time is that this study used more realistic but smaller consideration choice sets. Perhaps travel time plays a bigger role in choice set construction (i.e., in deciding which modes are feasible (Singleton, 2013)) than in a mode choice decision among actually considered alternatives. In studies with less restrictive (and less realistic) choice sets, a stronger travel time effect would likely show up in mode choice.

For travel activities, some results were consistent while others differed widely between the model with activity participation and the one with activity duration, although overall there were more negative associations than expected. In the activity participation model, several activities were negatively related to commute mode choice: participating in ICT activities, talking on the phone, reading print materials, and planning or navigating the trip. The only activities that gave people a higher odds of choosing a particular mode were personal grooming and (unexpectedly) doing nothing. In fact, people who reported doing nothing or expected to do nothing while commuting via a particular mode actually had about 3 times the odds $\left(\mathrm{e}^{\mathrm{B}}=3.36\right)$ of choosing that mode compared to someone doing some other activity. Notably, exercising or being physically active had a positive coefficient (with roughly twice the odds of choosing that mode), but this association was not statistically significant. 
The model using duration versions of travel activity variables found some similar associations. Talking on the phone and planning or navigating the trip were negatively associated with mode choice, while personal grooming had a positive association; ICT activities was still a negative but not significant factor. In this model, several additional activities had negative associations: passive activities, talking with strangers, and (expectedly but differently) doing nothing. People reporting spending or expecting to spend more time on these activities when using a particular mode were less likely to choose that mode.

One interesting implication of using activity durations in the utility equation is that values of time for activity participation can be calculated using the ratios of the travel activity duration and cost coefficients $\left(\mathrm{VTTS}=\mathrm{B}_{\mathrm{TA}} / \mathrm{B}_{\mathrm{CO}}\right)$. The ICLV model estimation results imply that, on average and at the margin, commuters would be willing to pay $19 \phi /$ min to avoid talking with strangers, $14 \phi /$ min to avoid planning or navigating the trip, $4 \not /$ min to avoid doing passive activities, and $37 \not / \mathrm{min}$ to avoid talking on the phone. Conversely, travelers might be willing to pay $46 \phi / \mathrm{min}$ for more time spent personal grooming, or $4 \phi / \mathrm{min}$ to avoid doing nothing. Of course, these interpretations are sensitive to small changes in the estimated parameters (particularly the cost coefficient) and should not be taken to mean that people have a literal willingness-to-pay for these things.

Results for the travel usefulness variable were neither consistent nor intuitive. In the model with activity participation, both "Wasted" and "Mostly useful" responses were positively and significantly associated with mode choice. In the model with activity duration, coefficient estimates were also in the positive direction, but only "Wasted" was statistically significant. These results indicate that people thinking that commuting by a 
particular mode is a waste of time were unexpectedly more likely to choose that mode, but also that considering a mode to be a good use of time might also have encouraged the use of that mode. See the Discussion section below for an interpretation of these results.

Measures of the PUT concept related to travel experiences were also significantly associated with commute mode choice. Notably, the "Commute satisfaction" construct obtained through the CFA model of the STS was a positive and significant factor. For scaling and identification purposes, the variance of this latent variable was fixed at 1.00, so coefficients can be interpreted as standardized effects. A one standard deviation increase in "Commute satisfaction" for any particular mode was associated with a greater than $250 \%$ increase $\left(e^{\mathrm{B}}-1=2.73\right)$ in the odds of using that mode in the model with activity participation; this was a more than $350 \%$ increase $\left(e^{\mathrm{B}}-1=3.79\right)$ in the model with activity duration. For comparison purposes, one standard deviation is about the difference between the median STS scores for people who walked vs. rode transit, and between those for people who bicycled vs. drove (see Chapter 5). As in previous analyses using this dataset (Chapter 4), travel liking appeared to be a positive factor, although it was significant only in the model with activity duration. Overall, people who liked or thought they would have liked commuting by a particular mode were more likely to choose that mode.

\subsection{Discussion}

This study is among the first to demonstrate revealed preference evidence consistent with measures of the positive utility of travel (PUT) concept having a direct impact on mode choice behavior. The significant associations between both travel activity and travel experience components and commute mode choice highlight the importance of 
these factors in travelers' mode choice decision-making processes. A rough magnitude of PUT's relative impact on mode choice in comparison to more traditional variables can be calculated by examining the proportions of DCA model deviance reduced by the addition of PUT-related variables. In both models with activity participation and activity duration variables, the LOS and T\&T variables contributed about $70 \%$ of the reduction in model deviance vs. the constants-only model. Thus, we can conclude that adding detailed and mode-varying measures of travel activities and travel experiences related to the PUT concept contributed around $30 \%$ of the explanatory power of the final mode choice models. In other words, adding PUT-related variables increased the explanatory power of the models by around $40 \%$ or more. This sizeable amount highlights the importance and value of accounting for these effects in a mode choice study.

\subsubsection{Travel activities}

Although measures of travel activity attributes of the PUT concept appear to have explained commute mode choices to a moderate degree, a closer inspection of specific types of travel-based multitasking tells a more complex story. Overall, the lack of positive significance of traditional productive multitasking activities (e.g., reading, listening to music, eating/drinking) suggests that people appeared not to value the ability to make productive use of their time spent commuting. In fact, the many negative associations between specific travel activities and mode choice might suggest that activity participation during travel may be more of a burden than a benefit. This conclusion is neither consistent with theory (see Chapter 2) nor easily interpretable. 
Yet, all hope for understanding the role of travel activity aspects of PUT on travel behavior is not lost. A closer inspection of the types of negatively associated activities could tell a different story: The things people report doing (or potentially doing) may be more the result of a coping mechanism to deal with a less-than-desirable commute than motivations for choosing a particular mode. Talking with strangers may be an uncomfortable experience for some, which could explain its negative association with mode choice. Similarly, doing or spending time planning or navigating could be somewhat of a burdensome task. Doing nothing is the quintessential "antiactivity" (Mokhtarian \& Salomon, 2001), so it makes sense that people would prefer spending less time doing nothing. As discussed in previous work (Chapter 4), some activities with negative coefficients - in this case, ICT activities, passive activities, and talking on the phone - may be more about "killing time" than making use of it. Looking out the window or checking email or social media may be ways for people to pass the time during what would otherwise be a long or boring commute. Given the choice between playing on a smartphone or doing nothing, people may indeed prefer to use their phone, but given the choice between reading the internet while on the bus or not using their phone when driving to work, they may choose to drive.

As mentioned in the results section, the ICLV model results showed an unexpected, confusing, and nonmonotonic association between travel usefulness and mode choice. Theory would suggest that travelers making or expecting to make greater use of their travel time via a particular mode would be more likely to choose that mode (see Chapter 2); instead, the opposite association was found. One potential explanation is that travel usefulness was strongly correlated with at least a subset of travel activities, although this 
rationale is not convincing for a couple of reasons. First, an earlier modeling effort found that few activities (either participation or duration) significantly predicted travel usefulness (Chapter 4). Second, preliminary model testing (not shown) suggested that removing the travel usefulness variable had minimal to no effects on the statistical significance of any other travel activity variable. Instead, it is more likely that the true association between travel usefulness and mode choice was confounded by other measures of the PUT concept. During the ICLV model building process (not shown), travel usefulness retained a positive (or statistically insignificant) association with mode choice only until the addition of travel experience variables. Earlier work found travel liking to be a strong predictor of travel usefulness (Chapter 4), and, when treated as continuous variables, the two measures were somewhat strongly correlated (0.61). There appears to be a significant amount of shared variance between the travel usefulness and travel liking variables, making it empirically challenging to distinguish the two concepts. This study adds further evidence to suggest that this single-item question of travel usefulness is not very useful in understanding travel activity aspects of the PUT concept (see Chapter 4) or in explaining travel behavior.

\subsubsection{Travel experiences}

The strong, consistently positive, and statistically significant association between "Commute satisfaction" and mode choice is a major finding: For possibly the first time, a validated multiple-item metric of SWB (the STS) has been found to positively influence mode choice. It appears that commuters may indeed consider and make mode choice decisions based on expectations of improvements in well-being as a result of the commute or at least expected differences in SWB across modes. Although this cross-sectional study 
cannot shed light on the causal nature of this relationship, there is reason to believe that this association may stand up to a longitudinal or experimental research approach. The instrument used to measure SWB, the STS, has been tested in a variety of contexts and its psychometric structure has held up (Chapter 5). Furthermore, this study specifically asked commuters to imagine what their experienced "Commute satisfaction" would have been had they used a different mode. Thus, this attribute of alternatives was measured (not modeled) in a consistent way for each considered mode. Finally, this finding is consistent with a growing body of theory and empirical evidence suggesting that the travel experience aspects of the PUT concept should play a role in mode choice decisionmaking and other travel behaviors (see Chapter 2).

One challenge, however, is in determining the relative magnitude of the effect of travel experience aspects of PUT on mode choice as compared to the impact of travel activities. In terms of reduced deviance (see Table 6.1), the travel experience variables appeared to be slightly more explanatory of mode choice than the travel experience variables in the ICLV model with activity participation but slightly less relevant in the activity duration model. Furthermore, when going from the model of Table 6.2 to the activity participation model of Table 6.3, the addition of PUT measures reduced the model deviance by less than the sum of adding each type individually. However, adding both travel activity and travel experience variables to generate the activity duration model (Table 6.4) reduced the model deviance by an amount greater than the sum of their parts (see Table 6.1). More investigation is needed to reconcile these empirical discrepancies.

The partial significance and nonsignificance of the travel liking variable was not surprising. Previous investigations with the same dataset found a strong correlation 
between the travel liking and travel usefulness measures, suggesting they may be parts of overlapping concepts (Chapter 4). However, this finding does suggest that travel liking, as a standalone measure of SWB or the travel experience aspects of PUT, may be poor suited to explaining mode choice behavior. It is somewhat discouraging that travel liking was not more significantly associated with mode choice, since it is an easily administered and commonly used metric for travel affect (Mokhtarian \& Salomon, 2001; Ory \& Mokhtarian, 2005; Turcotte, 2006). On the other hand, travel liking ratings were somewhat strongly correlated with CFA-predicted scores of "Commute satisfaction" (0.64), so the STS construct may have masked some of travel liking's potential effect. At the very least, even if travel liking is significantly and positively associated with commute mode choice, as a single measure of travel affect (see Chapter 2) it has limited ability to convey a wider array of emotions and feelings of fulfillment that comprise the full spectrum of travel experiences related to the PUT concept (see Chapter 5).

\subsubsection{Traveler characteristics}

The mode choice models provided insight not only about the role of PUT-related attributes, but their results also shed light on interpretations of socio-demographic characteristics. Some mode-specific findings were not surprising. For instance, people with mobility disabilities may find it more difficult to operate a vehicle (automobile or bicycle), so the result that they were less likely to use these operating modes and more likely to walk was not unexpected. Other expected findings point more towards multidirectional causal relationships than direct effects on mode choice. The fact that people owning more bicycles were more likely to cycle to work, and that people with a transit pass were more likely to 
commute by transit, likely indicates that these people were already inclined to bicycle and use transit (respectively) due to convenience, location, or other reasons. Their obtention of these mobility tools is more likely a reflection of their long-term average mode choice behavior than it is a direct causal factor in any one particular mode choice decision.

Instead, some of the cross-modal influences are more interesting. One example is the finding (especially in the model with activity participation) that people with a transit pass had 4-5 times the odds of walking, bicycling, and riding as an automobile passenger over driving. This association could be a reflection of locational effects: People who live and/or work in transit-accessible locations may also have better or more attractive nonauto commute options. Alternatively, perhaps mobility style preferences (Vij, Carrel, \& Walker, 2013) influenced this relationship: People who are predisposed to ride public transit may also prefer walking and bicycling to driving. (Both effects may operate through residential location choice (Schwanen \& Mokhtarian, 2005).) Another interesting finding is the positive association between owning more bicycles and commuting by walking and transit. Perhaps this relates to an unobserved nonmotorized or active travel mode preference among people who own bicycles. The positive association between owning bicycles and transit commuting is consistent with other research looking at synergies between bicycle and transit use; similar explanations have been posited (Singleton \& Clifton, 2014).

Associations between several socio-demographic characteristics and bicycling are also interesting and consistent with previous research. Many studies have documented the gender gap in bicycling: As found here, women in the U.S. are significantly less likely to cycle than their male counterparts, leading men to outnumber women on bicycles by 2:1 or even 3:1 (Krizek, Johnson, \& Tilahun, 2005; Pucher, Buehler, Merom, \& Bauman, 
2011). Research suggests a number of potential explanations for the gender gap, including differences in the perceptions of the safety of bicycling, childcare and home-based responsibilities, and other socio-normative gendered cultural factors (Garrard, Handy, \& Dill, 2012; Singleton \& Goddard, 2016). In addition, bicycling seems to have been associated with reduced economic and mobility means: living in multifamily housing, owning fewer cars, being younger, and having less income (not significant). This conclusion is consistent with previous research suggesting that men with more limited means (unlike women in the same situation) are more likely to bicycle (Singleton \& Goddard, 2016), so this finding is not surprising given the gender gap found in this study.

Other results point towards the roles of scheduling constraints and home/work responsibilities on mode choices. People with more children and who worked more hours each week were consistently more likely to drive and less likely to commute by walking, bicycling, transit, or as an auto passenger. At home, time spent on childcare responsibilities (including dropping kids off at school or daycare) may impose time constraints that encourage parents to consider travel time above all else and choose the fastest mode to work (often, driving). Similarly, people working longer hours may find it more challenging to fit other daily activities around their job responsibilities and may be less able to schedule their life in a way to optimize their commute, thus turning to driving. (This finding could also reflect a greater budget freedom—working more hours generates greater income, thus allowing the choice of the more expensive mode - although this effect may be less likely.) People with a flexible work schedule were also more likely to drive and less likely to walk, bicycle, or ride transit. Perhaps the flexibility afforded these people by their jobs or their employers lets them drive to work during off-peak times, thus avoiding congestion and 
stress. People who must work 9-to-5 jobs may not have this time-shifting luxury and may seek nonauto modes instead as a congestion-coping strategy. On the other hand, older working adults were more likely to choose to walk or ride transit, both slower modes, perhaps because they have fewer childcare or other responsibilities, more leisure time, and thus diminished incentives to economize on time. These findings suggest that a more holistic view of daily activity schedules and time constraints (Timmermans \& Zhang, 2009; Rasouli \& Timmermans, 2014d) would be useful for framing some aspects of mode choice behaviors.

Some findings require more investigation before coming to a firm conclusion. For instance, why are higher-income commuters so much more likely to choose to ride as auto passengers? This could be an artifact of the small sample size (only 30 subjects chose this mode), or it could be an interesting behavioral pattern. In addition, weather was surprisingly not a significant factor in mode choice: Precipitation on the travel day did not significantly deter people from walking or bicycling. Perhaps people in Portland are acclimated to rain, or maybe weather played a role in when considering what modes were feasible (similar to travel time as hypothesized in the Results section). Another possibility is that weather caused reporting biases, in that people who may have been sensitive to inclement weather chose not to answer the survey on such days. The data in this study may be unable to examine these potential explanations.

\subsubsection{Limitations and future work}

Although offering promising evidence about a potential link between trip-specific measures of the PUT concept (both travel activity and travel experience aspects) and 
commute mode choice, this study is but an initial step. There are many directions to take this work both through additional analyses utilizing the same dataset and also by extending this work and addressing some limitations in future research.

The most straightforward extension of this work would be to examine different and more complex specifications of the mode choice utility equation. The ICLV models presented in this study assumed linear, independent (among one another), and modeindependent effects of level-of-service attributes, travel activities, travel usefulness, "Commute satisfaction," and travel liking. Although mode-specific coefficients for travel usefulness and travel liking were not significant, interacting mode with both travel activities and the STS variable could be productive. Because some travel activities were either nearly exclusive to or prohibited by certain modes (e.g., exercising while walking and bicycling, reading print while driving), using generic coefficients obscured the fact that some of these were mode-specific associations; but, using a full set of alternative-specific coefficients would likely lead to empirical identification issues. More careful and selective interactions between some activities and select modes could yield results that are more intuitive and easier to interpret. Examining an interaction between the STS measure and mode might highlight whether or not positive aspects of the travel experience are more important factors for some modes as opposed to others. Interactions between travel activity and travel experience measures may also be interesting to explore.

Ortúzar and colleagues (Bahamonde-Birke et al., 2017; Ortúzar and Willumsen 2011) recommend examining many interactions between types of variables, especially when dealing with perceptual attributes like the PUT measures in this study. Specifically, interacting nonvarying socio-demographic traveler characteristics with the LOS and PUT 
variables that did vary across alternatives would be a way to examine systematic taste variation. Do younger travelers value having a positive commuting experience or the ability to check the internet more or less than older adults when considering different commuting modes? Do women's perceptions of "Commute satisfaction" affect mode choices in a different way than men's perceptions? Of course, the number of parameters involved with such interactions quickly explodes and offers pitfalls for model estimation, but a judicious examination of such interactions would likely be informative. Questions about the roles of time and schedule constraints on mode choice could be examined by interacting the variables for numbers of children, hours worked, and flexible work schedules with travel time. Interactions between travel time and travel experience aspects would also be a promising approach to investigate. (The models with activity duration variables effectively account for an interaction between travel time and activity participation, except using a more accurate measurement of the actual time spent doing each activity.) Nonlinear travel time specifications could also be useful; some evidence suggests that travel time may be positively valued up until around 15 minutes, after which it becomes a negative factor (Milakis et al., 2015; Wachs et al., 1993; Young \& Morris, 1981).

A major limitation of the ICLV models presented herein is the relative lack of variables to represent travel experience aspects of the PUT concept. As a single consolidation of information gathered from the STS, the "Commute satisfaction" variable represents a somewhat limited perspective of hedonic SWB. This study's dataset collected much more detailed assessments of affective and eudaimonic aspects of SWB, and earlier analyses identified several other relevant latent constructs: four factors for travel affect (“Distress," "Fear," “Attentiveness," and "Enjoyment”), four aspects of travel eudaimonia 
("Security," "Autonomy," "Confidence," and "Health"), and the three components of the STS ("Positive deactivation," "Positive activation," and "Cognitive evaluation") (see Chapter 5). It is expected that including additional travel experience measures would not only improve model goodness-of-fit statistics and increase the share attributable to PUT factors, but also present a more nuanced and behaviorally-relevant explanation of their associations with commute mode choice. The challenge here lies in model estimation. Most studies employing ICLV models utilize only one or two latent variables because traditional estimation approaches-such as were used in this study (maximum simulated likelihood)—are time consuming and easily and quickly encounter convergence problems (Daziano \& Bolduc, 2013); indeed, models with the three STS components in Biogeme ran into estimation and convergence issues. Estimating an ICLV model with eight or more correlated latent variables with binary indicators would be fraught with complications and would be unlikely to yield unbiased and efficient parameter estimates if it converged at all. New and more flexible ICLV model estimation approaches—like Bhat and Dubey's (2014) combination of a probit kernel formulation and maximum approximate composite marginal likelihood (MACML) inference - have been developed that are independent of the number of latent variables and offer much faster computations; unfortunately, they are currently unavailable in software packages and require writing custom codes and scripts. Improvements to software capabilities and advances in the area of ICLV modeling may diminish these estimation barriers in the future.

There could be useful extensions of this work to simultaneously examine mode choice and other additional relationships, mirroring the way in which ICLV models simultaneously analyze discrete choice and latent variable structures. A first step would be 
to include exogenous variables-like LOS and T\&T characteristics - as explanatory factors of travel activity and/or travel experience variables. Earlier analyses have examined these potential determinants of PUT measures, finding some significant and meaningful associations (see Chapter 4 and Chapter 5). Future work could consider jointly analyzing the choice set decision construction process alongside mode choice. This might illuminate factors that had less significant or strong associations with mode choice than expectedsuch as travel time and weather variables - yet still place a crucial role in the mode choice decision process through the restriction of feasible alternatives. The potential complicating role of the travel liking variable suggests a need to examine it more closely, such as by analyzing its relationship among the other measures of SWB in the travel domain.

Beyond simply testing various model specifications or increasing model complexity through joint and simultaneous estimation, future work should consider improving the measurement of both travel activity and travel experience aspects of the PUT concept. As discussed above, the travel usefulness variable does not appear to work very well for its intended purpose (as an overall assessment of the value of travel-based multitasking). Instead, more targeted questions about the quality of travel time use for various activities and reasons for activity participation during travel (Rosenfield \& Zhao, 2016) might be more useful for this purpose. More fundamentally (but mostly applicable to travel experience aspects), temporal issues involved in the measurement of PUT attributes - particularly the mismatch between asking people to report what they did and felt on a recent commute vs. asking them to imagine what they would have done or would have felt if they had used a different mode-warrants more careful consideration during the survey design and data collection process. Issues surrounding the comparability of 
retrospective vs. prospective and chosen vs. imagined assessments (Abou-Zeid \& BenAkiva, 2012) are complex and challenging to resolve. New techniques for nearinstantaneous self-reporting of feelings and activities through internet-connected mobile devices (Jariyasunant et al., 2015; Zhao et al., 2015) offer potential in this regard.

Finally, it would be useful to examine these measures of the PUT concept and test their relationships with mode choice outside of the geographic bounds of this study. A number of factors may have affected the generalizability of these results. For one, compared to most U.S. cities, bicycling conditions and networks in the Portland area are relatively safe, comfortable, and robust. Studies in areas with less-well-developed bicycle facilities or more hostile drivers and environments might find different strengths of association between measures of travel SWB and mode choice. Further, compared to other major urban areas across the globe, Portland does not have a large and long-distance railbased transit network, so most transit commuters are traveling relatively short distances on vehicles that are not designed for multitasking. A study in a place with more long-haul rail commuters and businesses travelers (e.g., Lyons et al., 2016), where people have a greater chance to get work done during their commute, might reveal a higher value placed upon productivity and travel-based multitasking. External studies in other geographies and cultural contexts would also help illuminate whether or not these patterns and relationships are universal or culturally dependent.

\subsubsection{Contributions and policy applications}

This study makes several contributions to travel behavior research. Notably, it offers one of the first empirical analyses of the association with mode choice of trip- 
specific measures of both travel activity and travel experience aspects of the positive utility of travel (PUT) concept. Using an ICLV framework to simultaneously estimate discrete choice and structural equation models, the results offer stronger evidence than could be obtained by either a sequential estimation process or by using fewer or highly correlated explanatory PUT variables. This study achieved its goals by using a unique revealed preference dataset, in which PUT measures - travel-based multitasking, travel usefulness, hedonic and eudaimonic subjective well-being (SWB), and travel liking - were collected not just for the chosen mode but also for considered alternative modes. This intensive task is rarely, if ever, done.

In terms of results, this study also yielded important interpretations about the role of the PUT concept in commute mode choice decisions. Travel-based multitasking was associated with mode choice, although results suggested that reported activity participation during travel could be more about coping with one's commute and killing time than about making productive use of time spent traveling. A higher-order construct of hedonic SWB, as measured by the Satisfaction with Travel Scale (STS), was shown to be significantly and positively associated with mode choice, suggesting that respondents may indeed have considered expected well-being when choosing a commute mode. These findings are consistent with the hypothesis that the PUT concept influences travel behavior (see Chapter 2). Overall, these PUT factors appeared to account for somewhere in the range of a $40 \%$ reduction in commute mode choice model deviance over traditional level-of-service and socio-demographic characteristics alone, a substantial amount that warrants their consideration and inclusion in future studies of travel behavior and mode choice. 
The findings of these analyses also offer important implications for transportation policy. The significant positive association between the "Commute satisfaction" construct and mode choice suggests that efforts to improve the traveling experience could be effective in achieving mode shifts towards more socially desirable behaviors. For example, making walking more enjoyable by providing safer street crossings, wider and more pleasant sidewalks, and enhancing streetscapes could make this mode more attractive. Similarly, reducing the stresses of bicycling through dedicated infrastructure, such as protected bike lanes (Monsere et al., 2014) and complete low-stress networks (Furth, Mekuria, \& Nixon, 2016), could increase the well-being of bicycle travelers and encourage more people to try cycling for transportation purposes. Efforts to make these healthy and active modes more friendly and fun, including by enhancing social connections through transportation, offers the potential to increase SWB and help achieve some of the nonauto modal goals of various jurisdictions (City of Portland \& Multnomah County, 2015). Because many instances of travel-based multitasking appear to be more closely related to killing time than using time productively, efforts to make commuting and other personal transportation situations less burdensome might also be of benefit. Public transit in particular could see investments in productivity-enhancing attributes such as WiFi or tray tables. In the short run, focusing on improving the quality of commuting and travel in general — and thus travelers' well-being — may be more likely to affect behavior than a focus on enhancing multitaskability, although helping travelers reduce the burden of their commutes could make them happier too.

In the long run, issues related to the PUT concept will likely increase in relevance with respect to mode choice behavior. The forthcoming development of semi- and fully- 
autonomous vehicles (AVs) may usher in a new era of personal transportation. A significant amount of brainpower and some hypothetical research using assumptions and simulations suggests that major travel behavior changes will likely occur because-by reducing the need for a physical operator-AVs offer the potential to multitask while traveling in nearly any way possible: eating, reading, watching a movie, playing a game, or even sleeping. Subjective values of travel time will likely be reduced - by how much is still highly uncertain - thus increasing the demand for automobile travel, potentially increasing congestion and sprawl (e.g., Childress et al., 2015). The results of this study suggest that reductions in time valuations may actually be smaller than imagined, as many people may engage in travel activities more to pass the time than to be productive. However, vehicle-miles-traveled may still increase because of AVs, but more because of improvements to travel well-being than from increases in productivity. By reducing the stresses of the driving task, allowing people to feel happier and more relaxed, and making commuting go more smoothly, AVs could increase SWB in ways that affect mode choice. How large this effect might be is an open question for future research. 


\section{Chapter 7 Conclusion}

\subsection{Summary}

This dissertation explored the "positive utility of travel" (PUT) concept and its relationship with mode choice. The PUT idea stands in contrast to the derived-demand paradigm of travel analysis, which assumes that travel is a means to an end (reaching destinations) and travel time is a disutility to be minimized. Instead, the PUT concept suggests that travel can provide additional benefits or be intrinsically motivated, such as through travel-based multitasking and increased sensations of well-being. This research sought to illuminate the PUT notion both theoretically and empirically. First, it reviewed and critiqued literature to provide a more rigorous conceptualization of a PUT and its components, and assessed the convincingness of existing PUT measures and evidence. Next, novel and original data on PUT attributes with respect to mode choice were collected for nearly 700 commuters in the Portland, OR, area. Finally, detailed data analyses constructed measurement models of travel experience aspects, identified potential determinants of PUT measures among trip and traveler characteristics, and examined associations between travel-based multitasking and satisfaction and commute mode choice

decisions. Robust measures of the PUT concept were found to vary by mode and to be significantly associated with mode choice.

This concluding section first highlights key findings of this study by returning to the research questions posed in the Introduction section. Next, it summarizes the major contributions of this work towards a greater knowledge of the PUT concept, its conceptualization and measurement, and its association with travel behavior. The research 
and policy implications of this study are then discussed, including those relevant to understanding potential impacts of future transportation technologies. This section concludes by presenting some limitations and opportunities for further research.

\subsection{Key findings}

This dissertation and the analyses contained within produced numerous key findings about the PUT concept, its measurement, its potential determinants, and its relationship with mode choice. These findings may best be summarized by revisiting the primary research questions and providing a retrospective assessment of their answers.

\section{What is the positive utility of travel (PUT) concept?}

a. Conceptually, how is the PUT idea defined and structured?

The literature review of Chapter 2 strengthened the theoretical support for the PUT concept. It defined a PUT as "any benefit(s) accruing to a traveler through the act of traveling," which could fall into at least one of three major areas: destination activities (travel as a derived demand), travel activities (travel-based multitasking), and travel experiences (travel subjective well-being). The review clarified the distinctions between each of these categories and demonstrated their foundation in and connection to issues such as utility maximization, motivation theory, multitasking, and subjective well-being (SWB).

\section{b. Empirically, how can a positive utility of travel be measured?}

The same literature review also examined and critiqued various ways to measure the multifaceted PUT concept. Questions directed at individual respondents that are more 
closely and conceptually linked to general PUT-related issues - those about desired travel amounts, excess travel indicators, and the teleportation test—seem to be most useful and provide more convincing evidence of a PUT than many aggregate observational methods. More useful still would be trip-based measures of the travel activity and travel experience PUT components. For travel activities, these include questions about activity participation or travel-based multitasking as well as the overall usefulness of time spent traveling. For travel experiences, measures are related to SWB: travel affect (emotions), travel eudaimonia (symbolism), the Satisfaction with Travel Scale, and overall travel liking. A least a few studies have examined each of these aspects, finding evidence consistent with the PUT hypothesis.

Based on these recommendations, an online questionnaire survey about mode choice was designed and administered to commuters in the Portland, OR, metropolitan area, as described in Chapter 3. The survey included detailed questions about travel-based multitasking, travel usefulness, subjective well-being, and travel liking while on a respondent's most recent commute trip. It also asked people to answer the same questions about what they would have done and would have felt for other modes they considered using. Nearly 700 people completed the survey; valid responses for each PUT section ranged from 680 to 624 .

Chapter 4 presents results for the travel activity aspects of the PUT concept. Respondents reported whether or not they participated in any of 23 activities while commuting. An exploratory factor analysis (EFA), designed to group similar activities, found only two groupings: "ICT" activities related to information and communication technologies - texting/emailing/messaging, reading electronically, and using social 
websites/apps - and passive activities, including viewing scenery or people watching and thinking/daydreaming. The most commonly reported travel activities were listening to music or the radio and the two passive activities. This study demonstrated that self-reported activity participation could be a useful way to measure the travel activity PUT component.

In contrast, Chapter 5 presents results for the travel experience aspects of the PUT concept. Several questions related to SWB in the travel domain were asked of respondents. Borrowing from the Positive and Negative Affect Schedule (Watson et al., 1988), two blocks of questions with 20 total items on a five-point Likert-type scale asked about travel affect, or the things people felt while commuting. An EFA and subsequent confirmatory factor analysis (CFA) generated four latent constructs related to travel affect: two negative ("Distress," "Fear") and two positive ("Attentiveness," "Enjoyment") factors. Three additional question blocks asked whether commuting fulfilled a desire for, expressed, or improved each of 22 items about travel eudaimonia. An EFA and CFA yielded four related constructs: "Security," "Autonomy," “Confidence," and "Health.” An existing travel-based instrument - the Satisfaction with Travel Scale (STS), made up of nine items measured on a seven-point semantic differential scale (Ettema et al., 2011) —was also administered with minor adjustments. A CFA confirmed the STS's three-factor structure, composed of one cognitive ("Cognitive evaluation") and two affective ("Positive deactivation," "Positive activation") latent variables. Overall, this study demonstrated the effectiveness of borrowing traditional psychological SWB measurement methods and using travel-specific items to collect data on PUT attributes related to subjective experiences of traveling. 


\section{What are potential determinants of a PUT?}

What personal and travel characteristics predict PUT measures?

Potential determinants of travel activities were examined in the analyses documented in Chapter 4. Binary logit models were estimated to predict participation in various activities and activity groups as a function of trip and traveler characteristics. Additionally, ordered logit models examined associations with a single measure of travel usefulness for trip and traveler characteristics as well as activity participation. Results found large and significant differences by commute mode. People who walked and bicycled found their commutes to be the most useful, apparently because they exercised and valued the physical activity. Auto drivers had the most wasteful commutes; most people only listened to audio. However, transit riders and auto passengers engaged in a greater number and variety of activities, including ICT-based ones. Although age was negatively associated with ICT activities, listening to music, and travel usefulness, few other sociodemographic attributes were consistently significant. Instead, traveler perceptions appeared to play a bigger role. Study findings suggest that some people indeed make use of their commute travel time through travel-based multitasking, while others travelers may instead be doing things just to kill time.

Associations between personal and transportation characteristics and the travel experience measures of the PUT concept are presented in Chapter 5. Structural equation modeling (SEM) was used to examine associations between trip/traveler characteristics and latent variables from the three CFAs. Again, modal differences loomed large. Walk and bicycle commuters had the highest overall travel satisfaction ratings and scored highly on other several constructs ("Enjoyment," "Autonomy," "Confidence," and "Health"). Auto 
drivers had the lowest overall ratings of SWB, in part because although they rated "Attentiveness" as high, they scored low on the "Health" factor and high in "Distress." In general, socio-demographic characteristics were usually less predictive of travel SWB than trip attributes and traveler perceptions. However, one finding (that remains partially unexplained) was that women consistently reported lower levels of positive affect, eudaimonia, and overall hedonic well-being from the commute. These results confirm that traveling experiences can strongly affect reports of SWB during travel, as supposed by the PUT notion.

\section{How do positive utilities of travel affect travel behavior?}

\section{How do PUT attributes impact travel mode choice?}

Chapter 6 describes analyses that explored the relationship between measures of the PUT concept and mode choice. An integrated choice and latent variable (ICLV) model was estimated using around 550 commute mode choice observations that measured PUT attributes for both the chosen mode and for at least one considered alternative. Several different specifications were tested, with the final model including level-of-service attributes, trip and traveler characteristics, activity participation, travel usefulness, the STS latent variable, and travel liking. Measures of travel-based multitasking were significantly associated with mode choice; however, results suggest that activity participation during travel may have been more about "killing time" than making use of it. For travel experiences, the STS construct was a significant and positive factor, suggesting that expectations of improvements to SWB likely play a role in mode choice decisions. Overall, adding PUT-related variables increased the explanatory power of the mode choice models 
by around $40 \%$ above traditional models using only travel time, cost, and traveler sociodemographics. These results are consistent with the hypothesized link between the PUT concept and travel behavior.

\subsection{Contributions}

This work makes major contributions to the travel behavior field, centered on the PUT concept but in the broad areas of theory, data collection, measurement, and evidence of potential determinants and effects on mode choice. In the area of theory, the literature review strengthens the field's conceptualization of what is and is not a PUT and of distinctions between its various components, adding value to existing reviews (Mokhtarian \& Salomon, 2001; Mokhtarian, Salomon, \& Singer, 2015; Salomon \& Mokhtarian, 1998). In some ways, the PUT concept pulls the word "utility" away from its economic connotations and back towards its psychological foundations. The review critiques evidence of the PUT concept, suggesting that some topics where PUT may be at worksuch as travel time budgets, excess commuting, and low rates of telecommuting-likely have other, more convincing non-PUT explanations. It also offers guidance towards the development and use of more direct and conclusive PUT measures.

The data collection process itself also involved novel elements. One notable aspect was the collection of both travel activity and travel experience aspects of the PUT concept in a single study. Most studies and surveys have focused on only one of these components at a time: either travel-based multitasking (e.g., Guo et al., 2015; Malokin et al., 2015) or topics related to subjective well-being (e.g., Mokhtarian et al., 2001; Smith, 2017). The second novel element was the solicitation of PUT attributes not only for the self-reported 
observed travel behavior (mode choice) but also for alternative behaviors (other modes considered). Few, if any, studies of the PUT concept have done this, likely because it can significantly increase respondent burden. Indeed, this decision made for a more intensive data collection process, but it also had the great advantage that no propensity-type models (e.g., Berliner et al., 2015) needed to be developed to generate PUT attributes of alternatives.

While the measures of travel activities used in this study are not unique, the detail with which travel experience aspects were measured is a distinctive characteristic. Notably, the development of new measurement models of travel affect and travel eudaimonia are major contributions. Despite well-established psychological methods and instruments for measuring hedonic SWB, these have rarely been applied in the context of traveling; instead, most studies use a handful of items or ad-hoc questions. Even fewer studies have tried to measure aspects related to travel eudaimonia in a more quantitative and systematic way. The fact that the CFA models of travel affect and travel eudaimonia were both intuitive and had at least adequate goodness-of-fits is an additional benefit. The confirmation of a three-factor structure to the Satisfaction with Travel Scale in a U.S. context makes another contribution to PUT measurement.

Although not as novel a contribution, this study also adds evidence pertaining to potential determinants of travel behavior aspects associated with the PUT concept. The analyses of Chapter 4 and Chapter 5 confirmed several relatively well-established findings about associations between PUT-related attributes and trip characteristics like travel time and mode. For instance, for travel activities, passive activities were common among all modes; car drivers were more likely to listen to music; and longer trips saw greater 
participation in some kinds of activities. For travel experience factors, measures of travel SWB were consistently rated more positively for walking and bicycling; and longer duration trips saw lower levels of SWB on average. The models confirmed the relative lack of consistent associations between socio-demographic traveler characteristics and PUT measures, although gender and age distinctions were among the most relevant and interesting.

Additionally, the more detailed measurement of PUT attributes in this study allowed for more nuanced modal distinctions on multiple dimensions. The most notable distinction was between more and less physically active modes: Active modes like walking and bicycling had higher STS scores and ratings on the "Enjoyment," "Confidence," and "Health" constructs, and people reported more useful commutes via these modes. Overall, this study provides evidence to suggest that walking and bicycling commuters enjoy gaining physical activity and value their use of travel time for exercise. Another distinction was between operating modes (bicycling, driving) and riding modes (transit, auto passenger). Users of riding modes had more useful commutes than auto drivers, in part because they could and did engage in a greater number and variety of potentially higherintensity activities. On the other hand, although operating modes high higher "Attentiveness" scores, other results suggested negative impacts of this mandatory focus on the traveling task: engagement in fewer and more passive activities, lower levels of "Positive deactivation," and higher reports of "Distress." Finally, there may be some evidence related to the personal exposure (Appleyard \& Ferrell, in press) experienced by users of various modes, with respect to social aspects-people walking, bicycling, and 
riding transit were more likely to talk to strangers — as well as safety and security: people bicycling had higher ratings of "Fear" and lower ratings of "Security."

This research also makes a strong contribution to our understanding of the PUTmode choice relationship. Notably, it offers one of the first empirical analyses of both travel activity and travel experience aspects in the context of mode choice, finding significant associations. Furthermore, including direct PUT measures for both chosen and alternative modes proved to be a distinct advantage over existing approaches to calculating attributes of mode choice alternatives, which either use stated preference methods to assign a limited number of PUT attributes to alternatives (e.g., van der Waerden et al., 2010) or model PUT propensities as a function of other nonvarying characteristics (e.g., Malokin et al., 2015). The use of an ICLV model framework to simultaneously estimate discrete choice, structural equation, and measurement model components also suggests the model results are more robust that could be obtained by alternative methodologies. Together, these characteristics imply that the significant associations found between PUT attributes and mode choice are not only among the first but also among the most robust available in the current literature.

\subsection{Research implications}

This dissertation and its findings have several implications for travel behavior research. Most significantly, by demonstrating that attributes related to the PUT concept may have an influence on mode choice behavior that is potentially large, it suggests that future studies would be wise to consider including PUT measures in data collection and analysis procedures. Researchers can use the literature review to select the most appropriate 
measures of travel activity and travel experience aspects and to make sure to avoid missing a particular component. They can use guidance provided in this document to ensure that survey questions and items are worded in such a way as to better elicit useful responses and reduce potential confounding influences.

The analyses also offer researchers the opportunity to better measure the PUT concept in future research, especially with respect to travel experience aspects. Future studies could take the measurement models of travel affect and travel eudaimonia — and a version of the STS that has been validated in a U.S. context-and use them to perform further testing or to analyze PUT with respect to other travel behaviors. For travel activities, the difficulties involved in using the travel usefulness question (including its overlap with travel liking) suggests that, instead, researchers should use alternative or more detailed questions about the quality of travel time use or reasons for activity participation (Rosenfield \& Zhao, 2016). Applying the PUT measurement guidance contained in this study should advance the state of the research field with respect to these topics. Indeed, better measurement of the positive aspects of traveling may also help to improve our understanding and estimates of other behavioral influences, such as values of travel time, perceptions of safety and security, and attitudes and perceptions.

\subsection{Policy implications}

Many policy implications flow from the results of this work. For travel demand analysis, the significance of PUT measures in the mode choice model suggests that travel behavior models that do not include such measures (which counts most models in the literature) suffer from omitted variable and/or endogeneity bias in which parameter 
estimates may not be accurate (Fernández-Antolín et al., 2016; Singleton \& Clifton, 2015). For example, the magnitude of the estimated travel time coefficient may be falsely attenuated or magnified, depending on whether unobserved PUT measures are positively or negatively correlated with travel time, respectively. Because this coefficient is central to the calculation of the value of travel time savings (VTTS) (Mackie et al., 2001), biased willingness-to-pay measures may yield inaccurate estimates of mode/route shifts or user benefits of mobility-enhancing projects. For instance, if planners of a new toll road use inflated estimates of VTTS that do not consider PUT impacts, they might overestimate demand for the new facility and overstate the public (and investor) benefits of such a project. Measuring and accounting for PUT-related factors in travel behavior models can help to mitigate these biases and produce more accurate parameter estimates and behavioral sensitivities. Indeed, adding measures of the PUT concept to the mode choice model slightly reduced the implied VTTS, which is consistent with evidence elsewhere in this study (Chapter 5) that travel SWB was negatively correlated with travel time.

In the long run, if researchers can successfully measure, predict, and translate the PUT concept into a forecasting model (a major endeavor), planning tools may be able to evaluate a much wider array of transportation projects, programs, and policies. These efforts could have the greatest benefits in terms of improving understandings of walking and bicycling demand, considering the apparently strong influence of nonutilitarian aspects like the benefits of exercise. Incorporating the PUT concept into travel demand models might increase the parity of models' abilities to analyze both motorized and nonmotorized modes. 
More broadly, one does not need a model to apply the lessons learned from this research to the design and evaluation of transportation policies. For instance, the many jurisdictions seeking to promote the use of nonautomobile modes for various reasons could turn (and have been turning) to policies that improve the travel activity and travel experience aspects of the PUT concept. Many of these policies seek not to increase the disutility or generalized cost of driving; instead, they increase the benefits or positive utility of nonauto modes. For transit modes, agency managers might leverage travelers' desires to multitask by adding on-board or station-area productivity amenities like tray tables, charging stations, or $\mathrm{WiFi}$; the transit experience could also be improved with more comfortable seating or reductions in crowding. Marketing transit by borrowing strategies from automobile marketing, in which cars are made to seem fun and exciting, could also help to evoke positive emotions and remind people of some of the non-instrumental motivations for riding public transit. Engineering interventions to make walking and bicycling safer and more comfortable - things like safer street crossings, wider and more pleasant sidewalks, enhanced human-scale streetscapes, protected bike lanes and intersections, and complete low-stress bicycle networks-could improve the travel experience enough to make these nonmotorized modes more attractive. The protected bike lanes in particular seem promising, as by separating conflicts with motorized road users they could likely reduce the relatively high ratings of "Fear" and "Distress" currently experienced by people bicycling (Monsere et al., 2014). Other interventions—which may or may not be desired, but follow logically from an understanding of the PUT conceptcould increase the nonexercise productivity of walking and bicycling: wider bike lanes to support side-by-side conversations (McIlvenny, 2014); countdown timers prior to bicycle 
traffic signal green indications to enable rapid phone use; "smartphone walking lanes" (Kaplan, 2015); or exploratory games like Pokémon Go. In this vein, encouragement efforts to make active travel modes more fun, friendly, social, and socially acceptable could also increase their use by improving feelings of "Enjoyment." All of these policies rely on the hypothesis that transportation behavior change is possible by altering the multitasking potential and/or the overall experience of travel via different modes. While the crosssectional analyses presented in this research cannot speak to this causal relationship, they do offer evidence consistent with this hypothesis, suggesting that people may indeed consider these PUT-related aspects when making mode choices and could potentially shift between modes if sufficiently enticed.

Many policies may also directly affect people's lives in a positive way without necessarily changing behavior, either by increasing productivity through travel time use or by improving health and well-being. Enhancing opportunities for walking and bicycling may not only make for a healthier and happier population but could also increase people's productivity by allowing more time spent engaged in transportation-related physical activity instead of in the gym. Providing more modal options might allow people to better optimize their commutes around considerations beyond travel time. Furthermore, results that point towards captive mode users and burdens imposed by long commutes suggest that improving the quality of service of existing modes - by providing more comfortable nonmotorized infrastructure, faster and less crowded transit vehicles, or less congested roadways - could decrease negative emotions from the travel experience. The relative importance of ICT-based activities for transit riders and auto passengers, many of whom appear to be doing things simply to pass the time, suggests that these commuters may 
benefit the most from future advances in ICT. In fact, transit agencies could take a cue from another nonoperating mode in which travelers rely on set schedules and routes: air travel. Many airplanes have amenities like WiFi, tray tables, and entertainment systems designed to facilitate productive work or relaxation, thus helping to mitigate the discomfort of sitting next to strangers in a cramped and crowded place. Overall, efforts to make traveling less burdensome could generate happier travelers. Results suggest that, at least in the short run, mode shifts may be stronger in response to enhancements to the quality of travel experiences than improvements in multitaskability.

In the long run, these findings have important implications for understanding and anticipating transportation futures. In the recent past, smartphones and in-vehicle "infotainment" systems have already made traveling more enjoyable and productive. The looming introduction of advanced semi- and fully-autonomous vehicles (AVs) portends potentially massive shifts in travel patterns, in a large part because AVs offer productivity benefits that make automobile use more attractive. Mental and physical resources currently dedicated to the driving task could be reallocated towards travel-based multitasking. Vehicle designs may likely continue to emphasize passenger comfort and entertainment. Thus, future "drivers" may act and feel more like today's auto and transit passengers: engaging in more types of activities and not feeling as stressed. While relevant today, it appears that the PUT concept will likely play an even more important role in transportation behaviors in the future.

This study offers some additional initial guidance towards better quantifying the potential travel behavior and mode shift impacts of a more fully-automated personal transportation system. Most existing models and simulation studies (e.g., Childress et al., 
2015) use speculative assumptions about the magnitude of reductions in the disutility of travel time due to $\mathrm{AV}$-induced increases in productivity, although some are in the $25-50 \%$ range. The results of this study suggest that reductions in time valuations due to increased travel-based multitasking may actually be more modest, because many people appear to be doing things more just to pass the time than to be productive. Instead, the stronger behavioral effect could be about improvements to SWB: Connected vehicle technology could make trips go more smoothly or reliably, and eliminating the need to operate a vehicle could reduce the stresses of driving, making people happier and more relaxed. These results also invite interesting thought experiments about who would and would not use AVs. Who will be more likely to purchase and use AVs: those who seek ultraproductive travel (Lyons \& Urry, 2005) and AVs as extensions of the office; or those who value leisure time and $\mathrm{AVs}$ as extensions of the living room? Will drivers with preferences for "Attentiveness" and "Freedom" turn to bicycling when they can no longer operate their own vehicles? These questions remain open, yet an understanding of the PUT concept can help us work towards their answers.

\subsection{Limitations and future work}

A number of additional analyses could make use of the rich dataset collected in this study to address some of the limitations and simplifying assumptions used in this dissertation. In the measurement of PUT-related attributes, the CFA models of the STS, travel affect, and travel eudaimonia could be examined for measurement invariance (given sufficient sample sizes), testing whether their structures vary across modes. Some of the latent travel experience variables were positively skewed ("Distress" and "Fear"), so using 
nonlinear link functions in the MIMIC model regressions could better represent their relationships with trip and traveler characteristics. Additionally, these latent variable predictors were excluded from the final ICLV model to simplify the estimation; including them could reveal the impact of PUT moderation and yield estimates of indirect and total effects on mode choice.

More sophisticated mode choice models could also be estimated. The final models included only a single STS construct; if computational issues could be overcome, there would likely be important and relevant policy implications of models that include each of the four constructs comprising the travel affect and travel eudaimonia concepts. In general, more complex specifications for the mode choice utility equation could also reveal behavioral sensitivities that currently remain hidden. There are valid arguments to be made (Bahamonde-Birke et al., 2017; Ortúzar and Willumsen 2011) for further examining systematic taste variation through interactions between PUT measures and travel mode, travel time, and socio-demographic characteristics. Nonlinear specifications of travel time could also be valuable to examine (Milakis et al., 2015).

Other data collected during this research process have not yet been fully analyzed and could enrich the analyses already completed. For example, no data from the Part II survey have yet been included in the mode choice model or models looking at potential PUT determinants, primarily because doing so would further restrict the sample size. This subsample of observations with a full dataset could be used to test some of the hypotheses discussed above, such as the roles of attitudes towards multitasking (polychronicity) on travel activity participation, or the effects of general satisfaction with life on assessments of SWB in the travel domain. The travel liking variable deserves an inspection and 
examination of its relationship with the other travel experience PUT attributes, since it has been more widely used in previous research (Ory \& Mokhtarian, 2005). Looking more closely at non-trip-specific measures of the PUT concept, including satisfaction with travel time and the teleportation test, could prove valuable, as these have been the focus of previous empirical studies (De Vos et al., 2016; Russell \& Mokhtarian, 2015). There were a series of questions in the Part I survey related to the value of travel time savings and marginal tradeoffs between work time and commute time that have yet to be analyzed and may be useful in a future mode choice model. Finally, it would be informative to jointly model the choice set generation process alongside mode choice decisions to see whether factors like travel time play a larger role in one or the other.

There are other limitations of this study that could be addressed by travel behavior researchers in the future. Many of these improvements revolve around better measurement of the PUT concept, including for both travel activities and travel experiences. The relatively poor performance of the travel usefulness measure suggests the need for additional questions about item use (Lyons et al., 2016), the quality of travel time use, and reasons for activity participation (Rosenfield \& Zhao, 2016). Further improvements to the measurement of travel activities include distinguishing between activities that are done while on a primary mode like public transit versus those done on access/egress modes or while waiting (Mishra et al., 2015), as well as validating self-reported activity participation through mixed methods combining travel surveys with observations (Guo et al., 2015). For travel experience measures, a more rigorous scale development process (DeVellis, 2016) is warranted. Developing longer lists of items pertaining to travel affect and eudaimonia, paring them down using multiple studies across different populations and contexts, and 
formally testing the resulting simple structures for reliability and validity would be a stronger way to approach the development of new travel-specific SWB instruments. More fundamental research is also needed. For travel activities, the underlying motivations for travel-based multitasking should be examined more closely in the context of daily activity patterns, scheduling, and constraints, which could yield important insights into the potential for shifting of activities between travel and non-travel settings. For travel experiences, anticipation and self-selection effects should also be investigated in future work. Studying the connections between commute SWB and well-being at home and on the job could also bear fruit, particularly regarding the possibility that travel (from workto-home especially) can facilitate psychological detachment and provide a time to recover from the stresses of one's job.

More generally, this study looked only at commuting; other trip purposes might exhibit different patterns or relationships with the PUT concept (Keseru et al., 2015; Mokhtarian \& Salomon, 2001). Practically, some unique characteristics of Portland may have made the results slightly less generalizable: Portland's bicycling network is relatively robust and safe, at least compared to other U.S. cities, while its transit network does not have the same types of long-haul train lines that are more conducive to productive multitasking. Fundamentally, there are conceptual and likely empirical differences between asking questions about what people did and felt while on a recent trip versus asking them to consider what they would have done and felt if using a different mode. These distinctions between retrospective, prospective, and hypothetical assessments of PUT-related aspects like SWB have been discussed (Abou-Zeid \& Ben-Akiva, 2014), yet there are no easy solutions. Despite this study's framing of the PUT concept in the 
utilitarian paradigm and analysis of its relationship with mode choice using discrete choice methods, there may be other theoretical and empirical approaches that are more relevant. For instance, the importance of well-being in this process suggests the potential for needsbased approaches to travel behavior analysis (Abou-Zeid \& Ben-Akiva, 2012), which would also be more consistent with psychological perspectives on behavioral motivation and recent work characterizing daily activity patterns (Arentze \& Timmermans, 2009). Finally, longitudinal analyses of these relationships with the PUT concept could help to illuminate some of the issues and questions surrounding causality and time precedence. Despite these limitations, this dissertation made significant strides to advance knowledge surrounding the relationships between measures of the positive utility of travel concept and mode choice. 


\section{References}

Abou-Zeid, M., \& Ben-Akiva, M. (2011). The effect of social comparisons on commute well-being. Transportation Research Part A: Policy and Practice, 45(4), 345-361. doi:10.1016/j.tra.2011.01.011

Abou-Zeid, M., \& Ben-Akiva, M. (2012). Well-being and activity-based models. Transportation, 39(6), 1189-1207. doi:10.1007/s11116-012-9387-8

Abou-Zeid, M., \& Ben-Akiva, M. (2014). Satisfaction and travel choices. In T. Gärling, D. Ettema, \& M. Friman (Eds.), Handbook of sustainable travel (pp. 53-65). New York, NY: Springer. doi:10.1007/978-94-007-7034-8_4

Abou-Zeid, M., Ben-Akiva, M., Bierlaire, M., Choudhury, C., \& Hess, S. (2010). Attitudes and value of time heterogeneity. In E. Van de Voorde \& T. Vanelslander (Eds.), Applied transport economics: A management and policy perspective (pp. 523-545). Brussels, BG: De Boeck.

Agrawal, A. W., Schlossberg, M., \& Irvin, K. (2008). How far, by which route and why? A spatial analysis of pedestrian preference. Journal of Urban Design, 13(1), 81-98. doi:10.1080/13574800701804074

Algers, S., Bergström, P., Dahlberg, M., \& Lindqvist Dillén, J. (1998). Mixed logit estimation of the value of travel time (No. 1998: 15). Uppsala, SE: Uppsala University. Retrieved from http://hdl.handle.net/10419/82839

Alfonzo, M. A. (2005). To walk or not to walk? The hierarchy of walking needs. Environment and Behavior, 37(6), 808-836. doi:10.1177/001391650427401 
American Strategies (AS) \& Myers Research \& Strategic Services (MRSS). (2013). National Community Preference Survey. Chicago, IL: NATIONAL ASSOCIATION OF REALTORS®.

Anable, J., \& Gatersleben, B. (2005). All work and no play? The role of instrumental and affective factors in work and leisure journeys by different travel modes. Transportation Research Part A: Policy and Practice, 39(2), 163-181. doi:10.1016/j.tra.2004.09.008

Andreev, P., Salomon, I., \& Pliskin, N. (2010). Review: State of teleactivities. Transportation Research Part C: Emerging Technologies, 18(1), 3-20. doi:10.1016/j.trc.2009.04.017

Appleyard, B. \& Ferrell, C. E. (in press). The influence of crime on active and sustainable travel: New geo-statistical methods and theories for understanding crime and mode choice. Journal of Transport \& Health. doi:10.1016/j.jth.2017.04.002

Archer, M., Paleti, R., Konduri, K., Pendyala, R., \& Bhat, C. (2013). Modeling the connection between activity-travel patterns and subjective well-being. Transportation Research Record: Journal of the Transportation Research Board, 2382, 102-111. doi:10.3141/2382-12

Arentze, T. A., \& Timmermans, H. J. P. (2009). A need-based model of multi-day, multiperson activity generation. Transportation Research Part B: Methodological, 43(2), 251-265. doi:10.1016/j.trb.2008.05.007

Bahamonde Birke, F. J. (2016). Modeling with the unobserved/unobservable: An in-depth analysis of modeling issues concerning the role of attitudes and perceptions in the decision making process (Unpublished doctoral dissertation). Technische Universität Berlin, Berlin, DE. doi:10.14279/depositonce-5085 
Bahamonde-Birke, F. J., Kunert, U., Link, H., \& Ortúzar, J. d. D. (2017). About attitudes and perceptions: finding the proper way to consider latent variables in discrete choice models. Transportation, 44, 475-493. doi:10.1007/s11116-015-9663-5

Bates, J. J. (1987). Measuring travel time values with a discrete choice model: A note. The Economic Journal, 97(386), 493-498. doi:10.2307/2232894

Bates, W. (2009). Gross national happiness. Asian-Pacific Economic Literature, 23(2), 116. doi:10.1111/j.1467-8411.2009.01235.x

Becker, G. S. (1965). A theory of the allocation of time. The Economic Journal, 75(299), 493-517. doi:10.2307/2228949

Ben-Akiva, M. E., \& Lerman, S. R. (1985). Discrete choice analysis: Theory and application to travel demand. Cambridge, MA: MIT Press.

Ben-Akiva, M., McFadden, D., Gärling, T., Gopinath, D., Walker, J., Bolduc, D., ... \& Polydoropoulou, A. (1999). Extended framework for modeling choice behavior. Marketing Letters, 10(3), 187-203. doi:10.1023/A:1008046730291

Ben-Akiva, M., Walker, J., Bernardino, A. T., Gopinath, D. A., Morikawa, T., \& Polydoropoulou, A. (2002). Integration of choice and latent variable models. In H. S. Mahmassani (Ed.), In perpetual motion: Travel behavior research opportunities and application challenges (pp. 431-470). Amsterdam, NL: Pergamon.

Bentham, J. (1789/1948). An introduction to the principle of morals and legislations. Oxford, UK: Blackwell.

Berliner, R. M., Malokin, A., Circella, G., \& Mokhtarian, P. L. (2015). Travel-based multitasking: Modeling the propensity to conduct activities while commuting. 
Presented at the 94th Annual Meeting of the Transportation Research Board, Washington, DC. Available from http://trid.trb.org/view.aspx?id=1338990

Bernardin, V. L., Koppelman, F., \& Boyce, D. (2009). Enhanced destination choice models incorporating agglomeration related to trip chaining while controlling for spatial competition. Transportation Research Record: Journal of the Transportation Research Board, 2132, 143-151. doi:10.3141/2132-16

Bhat, C. R., \& Dubey, S. K. (2014). A new estimation approach to integrate latent psychological constructs in choice modeling. Transportation Research Part B: Methodological, 67, 68-85. doi:10.1016/j.trb.2014.04.011

Bianco, M., \& Lawson, C. (1998). Trip-chaining, childcare, and personal safety: Critical issues in women's travel behavior. In US DOT (Ed.), Women's travel issues: Proceedings from the second national conference, October 1996 (Report No. FHWAPL-97-024) (pp. 121-143). Washington, DC: US Department of Transportation.

Biddle, S. J., \& Mutrie, N. (2007). Psychology of physical activity: Determinants, wellbeing and interventions. New York, NY: Routledge.

Bierlaire, M. (2016). PythonBiogeme: A short introduction (Report TRANSP-OR 160706). Lausanne, CH: Ecole Polytechnique Fédérale de Lausanne. Retrieved from http://biogeme.epfl.ch/documents.html

Bovy, P. H., \& Stern, E. (1990). Route choice: Wayfinding in transport networks. Hingham, MA: Kluwer Academic Publishers. doi:10.1007/978-94-009-0633-4

Bradley, M., Bergman, A., Lee, M., Greene, E., \& Childress, S. (2015). Predicting and applying differential response rates in address-based sampling for a household travel 
survey. Transportation Research Record: Journal of the Transportation Research Board, 2526, 119-125. doi:10.3141/2526-13

Broach, J., Dill, J., \& Gliebe, J. (2012). Where do cyclists ride? A route choice model developed with revealed preference GPS data. Transportation Research Part A: Policy and Practice, 46(10), 1730-1740. doi:10.1016/j.tra.2012.07.005

Bureau of Transportation Statistics (BTS). (2017). Table 4-23: Average fuel efficiency of U.S. light duty vehicles. Washington, DC: US Department of Transportation. Retrieved from

https://www.rita.dot.gov/bts/sites/rita.dot.gov.bts/files/publications/national_transport ation_statistics/html/table_04_23.html

Cambridge Systematics, Inc., Vanasse Hangen Brustlin, Inc., Gallop Corporation, Bhat, C. R., Shapiro Transportation Consulting, LLC, \& Martin/Alexiou/Bryson, PLLC. (2012). Travel demand forecasting: Parameters and techniques (NCHRP Report 716). Washington, DC: Transportation Research Board. Retrieved from https://www.nap.edu/catalog/14665/

Cao, J. (2013). The association between light rail transit and satisfactions with travel and life: evidence from Twin Cities. Transportation, 40(5), 921-933. doi:10.1007/s11116013-9455-8

Cao, J., \& Ettema, D. (2014). Satisfaction with travel and residential self-selection: How do preferences moderate the impact of the Hiawatha Light Rail Transit line? Journal of Transport and Land Use, 7(3), 93-108. doi:10.5198/jtlu.v7i3.485

Cao, X., \& Mokhtarian, P. L. (2005a). How do individuals adapt their personal travel? Objective and subjective influences on the consideration of travel-related strategies for 
San Francisco Bay Area commuters. Transport Policy, 12(4), 291-302. doi:10.1016/j.tranpol.2005.03.003

Cao, X., \& Mokhtarian, P. L. (2005b). How do individuals adapt their personal travel? A conceptual exploration of the consideration of travel-related strategies. Transport Policy, 12(3), 199-206. doi:10.1016/j.tranpol.2005.03.002

Cao, X., Mokhtarian, P. L., \& Handy, S. L. (2009). No particular place to go: An empirical analysis of travel for the sake of travel. Environment and Behavior, 41(2), 233-257. doi: $10.1177 / 0013916507310318$

Childress, S., Nichols, B., Charlton, B., \& Coe, S. (2015). Using an activity-based model to explore the potential impacts of automated vehicles. Transportation Research Record: Journal of the Transportation Research Board, 2493, 99-106. doi:10.3141/2493-11

Choo, S., Collantes, G. O., \& Mokhtarian, P. L. (2005). Wanting to travel, more or less: exploring the determinants of the deficit and surfeit of personal travel. Transportation, 32(2), 135-164. doi:10.1007/s11116-004-2219-8

Choo, S., \& Mokhtarian, P. L. (2008). How do people respond to congestion mitigation policies? A multivariate probit model of the individual consideration of three travelrelated strategy bundles. Transportation, 35(2), 145-163. doi:10.1007/s11116-007$9142-8$

Chorus, C. G., \& Kroesen, M. (2014). On the (im-) possibility of deriving transport policy implications from hybrid choice models. Transport Policy, 36, 217-222. doi:10.1016/j.tranpol.2014.09.001 
Circella, G., Mokhtarian, P. L., \& Poff, L. K. (2012). A conceptual typology of multitasking behavior and polychronicity preferences. electronic International Journal of Time Use Research, 9(1), 59-107. doi:10.13085/eijtur.9.1.59-107

Circella, G., Salgado, J. R., Mokhtarian, P. L., \& Diana, M. (2015). The impact of activities while traveling on the subjective valuation of travel time. Presented at Northwestern $\begin{array}{llll}\text { University, } & \text { Evanston, } & \text { IL. } & \text { Retrieved }\end{array}$ http://www.transportation.northwestern.edu/docs/2015/04.23.Mokhtarian.presentation .$p d f$

Cirillo, C., \& Axhausen, K. W. (2006). Evidence on the distribution of values of travel time savings from a six-week diary. Transportation Research Part A: Policy and Practice, 40(5), 444-457. doi:10.1016/j.tra.2005.06.007

City of Portland \& Multnomah County (2015). Climate action plan. Portland, OR: City of Portland. Retrieved from https://www.portlandoregon.gov/bps/66993

Clay, M. J., \& Mokhtarian, P. L. (2004). Personal travel management: The adoption and consideration of travel-related strategies. Transportation Planning and Technology, 27(3), 181-209. doi:10.1080/0308106042000226907

Clifton, K. J., Singleton, P. A., Muhs, C. D., \& Schneider, R. J. (2016). Development of destination choice models for pedestrian travel. Transportation Research Part A: Policy and Practice, 94, 255-265. doi:10.1016/j.tra.2016.09.017

Cohn, D. (2015). Census considers new approach to asking about race - by not using the term at all. Fact tank: News in the numbers. Washington, DC: Pew Research Center. Retrieved from http://www.pewresearch.org/fact-tank/2015/06/18/census-considersnew-approach-to-asking-about-race-by-not-using-the-term-at-all/ 
Cooley, D. (2016) googleway: Retrieves data from Google Maps APIs (Version 1.0.0) [Software]. Available from https://CRAN.R-project.org/package=googleway

Cummins, R. A., Eckersley, R., Pallant, J., Van Vugt, J., \& Misajon, R. (2003). Developing a national index of subjective wellbeing: The Australian Unity Wellbeing Index. Social Indicators Research, 64(2), 159-190. doi:10.1023/A:1024704320683

Curry, R. W. (2000). Attitudes toward travel: The relationships among perceived mobility, travel liking, and relative desired mobility (Unpublished master's thesis). University of $\begin{array}{llll}\text { California, } & \text { Davis, } & \text { CA. } & \text { Retrieved }\end{array}$ http://www.its.ucdavis.edu/research/publications/publication-detail/?pub_id=413

Dal Fiore, F., Mokhtarian, P. L., Salomon, I., \& Singer, M. E. (2014). "Nomads at last”? A set of perspectives on how mobile technology may affect travel. Journal of Transport Geography, 41, 97-106. doi:10.1016/j.jtrangeo.2014.08.014

Daziano, R. A., \& Bolduc, D. (2013). Covariance, identification, and finite-sample performance of the MSL and Bayes estimators of a logit model with latent attributes. Transportation, 40(3), 647-670. doi:10.1007/s11116-012-9434-5

DeSerpa, A. C. (1971). A theory of the economics of time. The Economic Journal, 97(386), 828-846. doi:10.2307/2230320

Delbosc, A. (2012). The role of well-being in transport policy. Transport Policy, 23, 2533. doi:10.1016/j.tranpol.2012.06.005

Deutsch-Burgner, K., Ravulaparthy, S., \& Goulias, K. (2014). Place happiness: its constituents and the influence of emotions and subjective importance on activity type and destination choice. Transportation, 41(6), 1323-1340. doi:10.1007/s11116-014$9553-2$ 
DeVellis, R. F. (2016). Scale development: Theory and applications. Thousand Oaks, CA: Sage Publications.

De Vos, J., Mokhtarian, P. L., Schwanen, T., Van Acker, V., \& Witlox, F. (2016). Travel mode choice and travel satisfaction: Bridging the gap between decision utility and experienced utility. Transportation, 43, 771-796. doi:10.1007/s11116-015-9619-9

De Vos, J., Schwanen, T., Van Acker, V., \& Witlox, F. (2013). Travel and subjective wellbeing: a focus on findings, methods and future research needs. Transport Reviews, 33(4), 421-442. doi:10.1080/01441647.2013.815665

De Vos, J., Schwanen, T., Van Acker, V., \& Witlox, F. (2015). How satisfying is the Scale for Travel Satisfaction? Transportation Research Part F: Traffic Psychology and Behaviour, 29, 121-130. doi:10.1016/j.trf.2015.01.007

De Vos, J., \& Witlox, F. (2016). Do people live in urban neighbourhoods because they do not like to travel? Analysing an alternative residential self-selection hypothesis. Travel Behaviour and Society, 4, 29-39. doi:10.1016/j.tbs.2015.12.002

Diana, M. (2008). Making the "primary utility of travel" concept operational: A measurement model for the assessment of the intrinsic utility of reported trips. Transportation Research Part A: Policy and Practice, 42(3), 455-474. doi:10.1016/j.tra.2007.12.005

Diener, E. (1984). Subjective well-being. Psychological Bulletin, 95(3), 542-575. doi:10.1037/0033-2909.95.3.542

Diener, E. D., Emmons, R. A., Larsen, R. J., \& Griffin, S. (1985). The satisfaction with life scale. Journal of Personality Assessment, 49(1), 71-75. doi:10.1207/s15327752jpa4901_13 
Diener, E., Lucas, R., Schimmack, U., \& Helliwell, J. (2009). Well-being for public policy. New York, NY: Oxford University Press. doi:10.1093/acprof:oso/9780195334074.001.0001

Diener, E., Wirtz, D., Tov, W., Kim-Prieto, C., Choi, D. W., Oishi, S., \& Biswas-Diener, R. (2010). New well-being measures: Short scales to assess flourishing and positive and negative feelings. Social Indicators Research, 97(2), 143-156. doi:10.1007/s11205-009-9493-y

Dill, J., \& McNeil, N. (2016). Revisiting the four types of cyclists: Findings from a national survey. Transportation Research Record: Journal of the Transportation Research Board, 2587, 90-99. doi:10.3141/2587-11

Dill, J., \& Mohr, C. D. (2010). Long-term evaluation of individualized marketing programs for travel demand management (Report OTREC-RR-10-08). Portland, OR: Oregon Transportation Research and Education Consortium. doi:10.15760/trec.132

Dinno, A. (2012). paran: Horn's test of principal components/factors (Version 1.5.1) [Software]. Available from https://CRAN.R-project.org/package=paran

Dong, Z., Mokhtarian, P. L., Circella, G., \& Allison, J. R. (2015). The estimation of changes in rail ridership through an onboard survey: Did free Wi-Fi make a difference to Amtrak's Capitol Corridor service? Transportation, 42(1), 123-142. doi:10.1007/s11116-014-9532-7

Duarte, A., Garcia, C., Giannarakis, G., Limão, S., Polydoropoulou, A., \& Litinas, N. (2010). New approaches in transportation planning: Happiness and transport economics. NETNOMICS: Economic Research and Electronic Networking, 11(1), 532. doi:10.1007/s11066-009-9037-2 
Dutta-Bergman, M. J. (2004). Primary sources of health information: Comparisons in the domain of health attitudes, health cognitions, and health behaviors. Health Communication, 16(3), 273-288. doi:10.1207/S15327027HC1603_1

Ellaway, A., Macintyre, S., Hiscock, R., \& Kearns, A. (2003). In the driving seat: Psychosocial benefits from private motor vehicle transport compared to public transport. Transportation Research Part F: Traffic Psychology and Behaviour, 6(3), 217-231. doi:10.1016/S1369-8478(03)00027-5

Ettema, D., Friman, M., Gärling, T., Olsson, L. E., \& Fujii, S. (2012). How in-vehicle activities affect work commuters' satisfaction with public transport. Journal of Transport Geography, 24, 215-222. doi:10.1016/j.jtrangeo.2012.02.007

Ettema, D., Gärling, T., Eriksson, L., Friman, M., Olsson, L. E., \& Fujii, S. (2011). Satisfaction with travel and subjective well-being: Development and test of a measurement tool. Transportation Research Part F: Traffic Psychology and Behaviour, 14(3), 167-175. doi:10.1016/j.trf.2010.11.002

Ettema, D., Gärling, T., Olsson, L. E., \& Friman, M. (2010). Out-of-home activities, daily travel, and subjective well-being. Transportation Research Part A: Policy and Practice, 44(9), 723-732. doi:10.1016/j.tra.2010.07.005

Ettema, D., Gärling, T., Olsson, L. E., Friman, M., \& Moerdijk, S. (2013). The road to happiness: Measuring Dutch car drivers' satisfaction with travel. Transport Policy, 27, 171-178. doi:10.1016/j.tranpol.2012.12.006

Ettema, D., \& Verschuren, L. (2007). Multitasking and value of travel time savings. Transportation Research Record: Journal of the Transportation Research Board, 2010, 19-25. doi:10.3141/2010-03 
Evans, A. W. (1972). On the theory of the valuation and allocation of time. Scottish Journal of Political Economy, 19(1), 1-17. doi:10.1111/j.1467-9485.1972.tb00504.x

Evans, G. W., \& Wener, R. E. (2006). Rail commuting duration and passenger stress. Health Psychology, 25(3), 408-412. doi:10.1037/0278-6133.25.3.408

Festinger, L. (1957). A theory of cognitive dissonance. Evanston, IL: Row \& Peterson.

Fosgerau, M. (2006). Investigating the distribution of the value of travel time savings. Transportation Research Part B: Methodological, 40(8), 688-707. doi:10.1016/j.trb.2005.09.007

Franzen, A., \& Meyer, R. (2010). Environmental attitudes in cross-national perspective: A multilevel analysis of the ISSP 1993 and 2000. European Sociological Review, 26(2), 219-234. doi:10.1093/esr/jcp018

Franzen, A., \& Vogl, D. (2013). Two decades of measuring environmental attitudes: A comparative analysis of 33 countries. Global Environmental Change, 23(5), 10011008. doi:10.1016/j.gloenvcha.2013.03.009

Freedman, O., \& Kern, C. R. (1997). A model of workplace and residence choice in twoworker households. Regional Science and Urban Economics, 27(3), 241-260. doi:10.1016/S0166-0462(96)02158-8

Frei, C., Mahmassani, H. S., \& Frei, A. (2015). Making time count: Traveler activity engagement on urban transit. Transportation Research Part A: Policy and Practice, 76, 58-70. doi:10.1016/j.tra.2014.12.007

Friman, M., Fujii, S., Ettema, D., Gärling, T., \& Olsson, L. E. (2013). Psychometric analysis of the satisfaction with travel scale. Transportation Research Part A: Policy and Practice, 48, 132-145. doi:10.1016/j.tra.2012.10.012 
Fritz, C., Yankelevich, M., Zarubin, A., \& Barger, P. (2010). Happy, healthy, and productive: The role of detachment from work during nonwork time. Journal of Applied Psychology, 95(5), 977-983. doi:10.1037/a0019462

Furth, P. G., Mekuria, M. C., \& Nixon, H. (2016). Network Connectivity for Low-Stress Bicycling. Transportation Research Record: Journal of the Transportation Research Board, 2587, 41-49. doi:10.3141/2587-06

Gardner, B., \& Abraham, C. (2007). What drives car use? A grounded theory analysis of commuters' reasons for driving. Transportation Research Part F: Traffic Psychology and Behaviour, 10(3), 187-200. doi:10.1016/j.trf.2006.09.004

Gärling, T., Gillholm, R., \& Gärling, A. (1998). Reintroducing attitude theory in travel behavior research: The validity of an interactive interview procedure to predict car use. Transportation, 25(2), 129-146. doi:10.1023/A:1005004311776

Garrard, J., Handy, S., \& Dill, J. (2012). Women and cycling. In J. Pucher \& R. Buehler (Eds.), City cycling (pp. 211-234). Cambridge, MA: The MIT Press.

GasBuddy. (2017). Portland Gas Prices. Boston, MA: GasBuddy. Retrieved from http://www.portlandgasprices.com

Gatersleben, B. (2014). Psychological motives for car use. In T. Gärling, D. Ettema, \& M. Friman (Eds.), Handbook of sustainable travel (pp. 53-65). New York, NY: Springer. doi:10.1007/978-94-007-7034-8_6

Gatersleben, B., \& Uzzell, D. (2007). Affective appraisals of the daily commute comparing perceptions of drivers, cyclists, walkers, and users of public transport. Environment and Behavior, 39(3), 416-431. doi:10.1177/0013916506294032 
GenIUSS Group. (2014). Best practices for asking questions to identify transgender and other gender minority respondents on population-based surveys. Los Angeles, CA: The Williams Institute. Retrieved from http://williamsinstitute.law.ucla.edu/wpcontent/uploads/geniuss-report-sep-2014.pdf

Gim, T. H. T. (2015). The relationship between land use and automobile travel utility: A multiple indicators multiple causes approach. Transportation Research Part D: Transport and Environment, 41, 188-204. doi:10.1016/j.trd.2015.10.004

Giuliano, G., \& Small, K. A. (1995). Alternative strategies for coping with traffic congestion. In H. Giersch (Ed.), Urban agglomeration and economic growth (pp. 199225). Heidelberg, DE: Springer-Verlag. doi:10.1007/978-3-642-79397-4_9

Golob, T. F. (2003). Structural equation modeling for travel behavior research. Transportation Research Part B: Methodological, 37(1), 1-25. doi:10.1016/S01912615(01)00046-7

Gonzales, J. (2017). Using Google Maps API and R. GitHub. Retrieved from https://gist.github.com/josecarlosgonz/6417633

González, R. M. (1997). The value of time: A theoretical review. Transport Reviews, 17(3), 245-266. doi:10.1080/01441649708716984

Google. (2017a). Google Maps Geocoding API. Mountain View, CA: Google. Retrieved from https://developers.google.com/maps/documentation/geocoding

Google. (2017b). Google Places API Web Service Place Search. Mountain View, CA: Google. Retrieved from https://developers.google.com/places/web-service/search

Google. (2017c). Google Maps Directions API. Mountain View, CA: Google. Retrieved from https://developers.google.com/maps/documentation/directions 
Guo, Z., Derian, A., \& Zhao, J. (2015). Smart devices and travel time use by bus passengers in Vancouver, Canada. International Journal of Sustainable Transportation, 9(5), 335347. doi:10.1080/15568318.2013.784933

Hamilton, B. W. (1989). Wasteful commuting again. The Journal of Political Economy, 97(6), 1497-1504. doi:10.1086/261665

Hamilton, B. W., \& Röell, A. (1982). Wasteful commuting. The Journal of Political Economy, 90(5), 1035-1053. doi:10.1086/261107

Handy, S., Weston, L., \& Mokhtarian, P. L. (2005). Driving by choice or necessity? Transportation Research Part A: Policy and Practice, 39(2), 183-203. doi:10.1016/j.tra.2004.09.002

Hensher, D. A. (2010). Hypothetical bias, choice experiments and willingness to pay. Transportation Research Part B: Methodological, 44(6), 735-752. doi:10.1016/j.trb.2009.12.012

Harris, D. A. (1999). The stories, the statistics, and the law: Why driving while black matters. Minnesota Law Review, 84(2), 265-326.

He, S. Y., \& Hu, L. (2015). Telecommuting, income, and out-of-home activities. Travel Behaviour and Society, 2(3), 131-147. doi:10.1016/j.tbs.2014.12.003

He, M., Zhao, S., \& He, M. (2016). Tolerance threshold of commuting time: Evidence from Kunming, China. Journal of Transport Geography, 57, 1-7. doi:10.1016/j.jtrangeo.2016.09.007

Hess, S., Bierlaire, M., \& Polak, J. W. (2005). Estimation of value of travel-time savings using mixed logit models. Transportation Research Part A: Policy and Practice, 39(2), 221-236. doi:10.1016/j.tra.2004.09.007 
Hess, S., Train, K. E., \& Polak, J. W. (2006). On the use of a modified latin hypercube sampling (MLHS) method in the estimation of a mixed logit model for vehicle choice.

Transportation Research Part B: Methodological, 40(2), 147-163. doi:10.1016/j.trb.2004.10.005

Hiscock, R., Macintyre, S., Kearns, A., \& Ellaway, A. (2002). Means of transport and ontological security: Do cars provide psycho-social benefits to their users? Transportation Research Part D: Transport and Environment, 7(2), 119-135. doi:10.1016/S1361-9209(01)00015-3

Houseman, G. L. (1979). The right of mobility. Port Washington, NY: Kennikat Press.

Hu, L. T., \& Bentler, P. M. (1999). Cutoff criteria for fit indexes in covariance structure analysis: Conventional criteria versus new alternatives. Structural Equation Modeling: A Multidisciplinary Journal, 6(1), 1-55. doi:10.1080/10705519909540118

Hupkes, G. (1982). The law of constant travel time and trip-rates. Futures, 14(1), 38-46. doi:10.1016/0016-3287(82)90070-2

Jain, J., \& Lyons, G. (2008). The gift of travel time. Journal of Transport Geography, 16(2), 81-89. doi:10.1016/j.jtrangeo.2007.05.001

Jakobsson Bergstad, C., Gamble, A., Gärling, T., Hagman, O., Polk, M., Ettema, D., ... \& Olsson, L. E. (2011). Subjective well-being related to satisfaction with daily travel. Transportation, 38(1), 1-15. doi:10.1007/s11116-010-9283-z

Jara-Díaz, S. R. (2000). Allocation and valuation of travel time savings. In D. A. Hensher \& K. J. Button (Eds.), Handbooks of transport modeling (pp. 303-319). Oxford, UK: Pergamon. 
Jara-Díaz, S. R., \& Guevara, C. A. (2003). Behind the subjective value of travel time savings. Journal of Transport Economics and Policy, 37(1), 29-46. Retrieved from http://www.jstor.org/stable/20053921

Jariyasunant, J., Abou-Zeid, M., Carrel, A., Ekambaram, V., Gaker, D., Sengupta, R., \& Walker, J. L. (2015). Quantified traveler: Travel feedback meets the cloud to change behavior. Journal of Intelligent Transportation Systems, 19(2), 109-124. doi:10.1080/15472450.2013.856714

Johnson, M. B. (1966). Travel time and the price of leisure. Economic Inquiry, 4(2), 135145. doi:10.1111/j.1465-7295.1966.tb00941.x

Jones, P. M. (1978). Destination choice and travel attributes. In D. Hensher \& Q. Dalvi (Eds.), Determinants of travel choice (pp. 266-311). Westmead, UK: Saxon House.

Kahneman, D. (2000). Evaluation by moments: Past and future. In D. Kahneman \& A. Tversky (Eds.), Choices, values, and frames (pp. 693-708). New York, NY: Cambridge University Press.

Kahneman, D., Wakker, P. P., \& Sarin, R. (1997). Back to Bentham? Explorations of experienced utility. The Quarterly Journal of Economics, 112(2), 375-405. doi:10.1162/003355397555235

Kanaroglou, P. S., Higgins, C. D., \& Chowdhury, T. A. (2015). Excess commuting: A critical review and comparative analysis of concepts, indices, and policy implications. Journal of Transport Geography, 44, 13-23. doi:10.1016/j.jtrangeo.2015.02.009

Kaplan, S. (2015, June 17). Texting while walking? There's a lane for that. Washington Post. Retrieved from https://www.washingtonpost.com/news/morningmix/wp/2015/06/17/texting-while-walking-theres-a-lane-for-that/ 
Kenyon, S. (2006). The 'accessibility diary': Discussing a new methodological approach to understand the impact of Internet use upon personal travel and activity participation. Journal of Transport Geography, 14(2), 123-134. doi:10.1016/j.jtrangeo.2005.10.005

Kenyon, S. (2008). Internet use and time use: The importance of multitasking. Time \& Society, 17(2-3), 283-318. doi:10.1177/0961463X08093426

Kenyon, S. (2010). What do we mean by multitasking? Exploring the need for methodological clarification in time use research. electronic International Journal of Time Use Research, 7(1), 42-60. doi:10.13085/eIJTUR.7.1.42-60

Kenyon, S., \& Lyons, G. (2007). Introducing multitasking to the study of travel and ICT: Examining its extent and assessing its potential importance. Transportation Research Part A: Policy and Practice, 41(2), 161-175. doi:10.1016/j.tra.2006.02.004

Keseru, I., Bulckaen, J., Macharis, C., Minnen, J., Gloreiux, I., \& van Tienoven, T. P. (2015). Is travel time wasted? Evidence from a time use survey in Flanders, Belgium. Presented at the 14th International Conference on Travel Behaviour Research, Old Windsor, UK.

Kim, S. N., Choo, S., \& Mokhtarian, P. L. (2015). Home-based telecommuting and intrahousehold interactions in work and non-work travel: A seemingly unrelated censored regression approach. Transportation Research Part A: Policy and Practice, 80, 197214. doi:10.1016/j.tra.2015.07.018

Kim, K., Rousseau, G., Freedman, J., \& Nicholson, J. (2015). The travel impact of autonomous vehicles in metro Atlanta through activity-based modeling. Presented at the 15th TRB National Planning Applications Conference, Atlantic City, NJ. 
Kitamura, R. (1988). An evaluation of activity-based travel analysis. Transportation, 15(1), 9-34. doi:10.1007/BF00167973

Kline, R. B. (2016). Principles and practice of structural equation modeling (4th ed.). New York, NY: The Guilford Press.

König, C. J., \& Waller, M. J. (2010). Time for reflection: A critical examination of polychronicity. Human Performance, 23(2), 173-190. doi:10.1080/08959281003621703

Koppelman, F. S., \& Hauser, J. R. (1978). Destination choice behavior for non-groceryshopping trips. Transportation Research Record, 673, 157-165. Retrieved from http://www.jstor.org/stable/3150810

Koslowsky, M., Kluger, A. N., \& Reich, M. (1995). Commuting stress: Causes, effects, and methods of coping. New York, NY: Plenum Press. doi:10.1007/978-1-4757-97657

Krizek, K. J., Johnson, P. J., \& Tilahun, N. (2005). Gender differences in bicycling behavior and facility preferences. Research on Women's Issues in Transportation: Report of a Conference, 2, 31-40. Retrieved from http://onlinepubs.trb.org/onlinepubs/conf/cp35v2.pdf\#page $=41$

LaJeunesse, S., \& Rodríguez, D. A. (2012). Mindfulness, time affluence, and journeybased affect: Exploring relationships. Transportation Research Part F: Traffic Psychology and Behaviour, 15(2), 196-205. doi:10.1016/j.trf.2011.12.010

Lawrence, C. T., Zhou, J. L., \& Tits, A. L. (1994). User's guide for CFSQP Version 2.0: AC code for solving (large scale) constrained nonlinear (minimax) optimization problems, generating iterates satisfying all inequality constraints (Report TR-94-16). 
College Park, MD: University of Maryland. Retrieved from http://hdl.handle.net/1903/5496

Levin, M. W., \& Boyles, S. D. (2015). Effects of autonomous vehicle ownership on trip, mode, and route choice. Transportation Research Record: Journal of the Transportation Research Board, 2493, 29-38. doi:10.3141/2493-04

Loo, L. Y. L., Corcoran, J., Mateo-Babiano, D., \& Zahnow, R. (2015). Transport mode choice in South East Asia: Investigating the relationship between transport users' perception and travel behaviour in Johor Bahru, Malaysia. Journal of Transport Geography, 46, 99-111. doi:10.1016/j.jtrangeo.2015.06.011

Loukaitou-Sideris, A., \& Fink, C. (2009). Addressing women's fear of victimization in transportation settings: A survey of U.S. transit agencies. Urban Affairs Reviews, 44(4), 554-587. doi:10.1177/1078087408322874

Lyons, G., Jain, J., \& Holley, D. (2007). The use of travel time by rail passengers in Great Britain. Transportation Research Part A: Policy and Practice, 41(1), 107-120. doi:10.1016/j.tra.2006.05.012

Lyons, G., Jain, J., Susilo, Y., \& Atkins, S. (2013). Comparing rail passengers' travel time use in Great Britain between 2004 and 2010. Mobilities, 8(4), 560-579. doi:10.1080/17450101.2012.743221

Lyons, G., Jain, J., \& Weir, I. (2016). Changing times-A decade of empirical insight into the experience of rail passengers in Great Britain. Journal of Transport Geography, 57, 94-104. doi:10.1016/j.jtrangeo.2016.10.003

Lyons, G., \& Urry, J. (2005). Travel time use in the information age. Transportation Research Part A: Policy and Practice, 39(2), 257-276. doi:10.1016/j.tra.2004.09.004 
Ma, K. R., \& Banister, D. (2006). Excess commuting: A critical review. Transport Reviews, 26(6), 749-767. doi:10.1080/01441640600782609

MacCallum, R. C., Browne, M. W., \& Sugawara, H. M. (1996). Power analysis and determination of sample size for covariance structure modeling. Psychological Methods, 1(2), 130-149. doi:10.1037/1082-989X.1.2.130

MacDonald, H. I. (1999). Women's employment and commuting: Explaining the links. Journal of Planning Literature, 13(3), 267-283. doi:10.1177/08854129922092397

Mackie, P. J., Jara-Díaz, S., \& Fowkes, A. S. (2001). The value of travel time savings in evaluation. Transportation Research Part E: Logistics and Transportation Review, 37(2), 91-106. doi:10.1016/S1366-5545(00)00013-2

Malokin, A., Circella, G., \& Mokhtarian, P. L. (2015). How do activities conducted while commuting influence mode choice? Testing public transportation advantage and autonomous vehicle scenarios. Presented at the 94th Annual Meeting of the Transportation Research Board, Washington, DC. Available from http://trid.trb.org/view.aspx $? \mathrm{id}=1336974$

Mann, E., \& Abraham, C. (2006). The role of affect in UK commuters' travel mode choices: An interpretative phenomenological analysis. British Journal of Psychology, 97(2), 155-176. doi:10.1348/000712605X61723

Mao, Z., Ettema, D., \& Dijst, M. (2015). Commuting trip satisfaction in Beijing: Exploring the influence of multimodal behaviour and modal flexibility. Presented at the 14th International Conference on Travel Behaviour Research, Old Windsor, UK.

Marchetti, C. (1994). Anthropological invariants in travel behavior. Technological Forecasting and Social Change, 47(1), 75-88. doi:10.1016/0040-1625(94)90041-8 
Martin, A., Goryakin, Y., \& Suhrcke, M. (2014). Does active commuting improve psychological wellbeing? Longitudinal evidence from eighteen waves of the British Household Panel Survey. Preventive Medicine, 69, 296-303. doi:10.1016/j.ypmed.2014.08.023

Maslow, A. H. (1943). A theory of human motivation. Psychological Review, 50(4), 370396. doi:10.1037/h0054346

Maslow, A. H. (1954). Motivation and personality. New York, NY: Harper \& Brothers.

McFadden, D. (2001a). Disaggregate behavioral travel demand's RUM side: A 30-year retrospective. In D. Hensher (Ed.), Travel behavior research: The leading edge. Oxford, UK: Pergamon Press.

McFadden, D. (2001b). Economic choices. The American Economic Review, 91(3), 351378. doi:10.1257/aer.91.3.351

McIlvenny, P. (2014). Vélomobile formations-in-action: Biking and talking together. Space and Culture, 17(2), 137-156. doi:10.1177/1206331213508494

Milakis, D., Cervero, R., van Wee, B., \& Maat, K. (2015). Do people consider an acceptable travel time? Evidence from Berkeley, CA. Journal of Transport Geography, 44, 76-86. doi:10.1016/j.jtrangeo.2015.03.008

Mishra, G. S., Mokhtarian, P. L., \& Widaman, K. F. (2015). An empirical investigation of attitudes toward waiting on the part of Northern California commuters. Travel Behaviour and Society, 2(2), 78-87. doi:10.1016/j.tbs.2014.09.002

Mokhtarian, P. L. (1990). A typology of relationships between telecommunications and transportation. Transportation Research Part A: General, 24(3), 231-242. doi:10.1016/0191-2607(90)90060-J 
Mokhtarian, P. L. (2002). Telecommunications and travel: The case for complementarity. Journal of Industrial Ecology, 6(2), 43-57. doi:10.1162/108819802763471771

Mokhtarian, P. L. (2005). Travel as a desired end, not just a means. Transportation Research Part A: Policy and Practice, 39(2-3), 93-96. doi:10.1016/j.tra.2004.09.005

Mokhtarian, P. (2009). If telecommunication is such a good substitute for travel, why does congestion continue to get worse? Transportation Letters, 1(1), 1-17. doi:10.3328/TL.2009.01.01.1-17

Mokhtarian, P. L. (2014). What good is it? Reflections on the utility of travel in a resourceconstrained era. Presented at the 8th Annual Martin Wachs Lecture, UCLA Luskin School of Public Affairs, Los Angeles, CA. Retrieved from https://www.youtube.com/watch?v=RSVWPIyf7C0

Mokhtarian, P. L. (in progress). Subjective well-being and travel: Retrospect and prospect. Available from the author.

Mokhtarian, P. L., \& Chen, C. (2004). TTB or not TTB, that is the question: A review and analysis of the empirical literature on travel time (and money) budgets. Transportation Research Part A: Policy and Practice, 38(9), 643-675. doi:10.1016/j.tra.2003.12.004

Mokhtarian, P. L., Papon, F., Goulard, M., \& Diana, M. (2015). What makes travel pleasant and/or tiring? An investigation based on the French National Travel Survey. Transportation, 42(6), 1103-1128. doi:10.1007/s11116-014-9557-y

Mokhtarian, P. L., \& Salomon, I. (1994). Modeling the choice of telecommuting: Setting the context. Environment and Planning A, 26, 749-766. doi:10.1068/a260749 
Mokhtarian, P. L., \& Salomon, I. (1997). Modeling the desire to telecommute: The importance of attitudinal factors in behavioral models. Transportation Research Part A: Policy and Practice, 31(1), 35-50. doi:10.1016/S0965-8564(96)00010-9

Mokhtarian, P. L., \& Salomon, I. (1999). Travel for the fun of it. ACCESS Magazine, 15, 26-31. Retrieved from http://escholarship.org/uc/item/8344g0q2

Mokhtarian, P. L., \& Salomon, I. (2001). How derived is the demand for travel? Some conceptual and measurement considerations. Transportation Research Part A: Policy and Practice, 35(8), 695-719. doi:10.1016/S0965-8564(00)00013-6

Mokhtarian, P. L., \& Salomon, I. (2002). Emerging travel patterns: Do telecommunications make a difference? In H. S. Mahmassani (Ed.), In perpetual motion: Travel behavior research opportunities and application challenges (pp. 143-182). Oxford, UK: Elsevier.

Mokhtarian, P. L., Salomon, I., \& Redmond, L. S. (2001). Understanding the demand for travel: It's not purely 'derived'. Innovation: The European Journal of Social Science Research, 14(4), 355-380. doi:10.1080/13511610120106147

Mokhtarian, P. L., Salomon, I., \& Singer, M. E. (2015). What moves us? An interdisciplinary exploration of reasons for traveling. Transport Reviews, 35(3), 250274. doi:10.1080/01441647.2015.1013076

Monsere, C., Dill, J., McNeil, N., Clifton, K., Foster, N., Goddard, T., ... \& Parks, J. (2014). Lessons from the green lanes: Evaluating protected bike lanes in the U.S. (Report NITC-RR-583). Portland, OR: National Institute for Transportation and Communities. Retrieved from http://trec.pdx.edu/research/project/583/ 
Morikawa, T., Ben-Akiva, M., \& McFadden, D. (2002). Discrete choice models incorporating revealed preferences and psychometric data. Econometric Models in Marketing, 16, 29-55. doi:10.1016/s0731-9053(02)16003-8

Morris, E. A., \& Guerra, E. (2015a). Mood and mode: Does how we travel affect how we feel? Transportation, 42(1), 25-43. doi:10.1007/s11116-014-9521-x

Morris, E. A., \& Guerra, E. (2015b). Are we there yet? Trip duration and mood during travel. Transportation Research Part F: Traffic Psychology and Behaviour, 33, 38-47. doi:10.1016/j.trf.2015.06.003

Mumford, L. (1961). The city in history: Its origins, its transformations, and its prospects. New York, NY: Harcourt, Brace \& World, Inc.

Muthén, B. O., du Toit, S. H. C., \& Spisic, D. (1997). Robust inference using weighted least squares and quadratic estimating equations in latent variable modeling with categorical and continuous outcomes. Los Angeles, CA: University of California at Los Angeles. Retrieved from https://www.statmodel.com/download/Article_075.pdf

National Oceanic and Atmospheric Administration (NOAA). (2017). Climate data online. Washington, DC: National Centers for Environmental Information. Retrieved from https://www.ncdc.noaa.gov/cdo-web/

Nederhof, A. J. (1985). Methods of coping with social desirability bias: A review. European Journal of Social Psychology, 15(3), 263-280. doi:10.1002/ejsp.2420150303

Neufeld, A. J., \& Mokhtarian, P. L. (2012). A survey of multitasking by Northern California commuters: Description of the data collection process (Report UCD-ITS-RR-12-32). Davis, CA: Institute of Transportation Studies. Retrieved from 
https://itspubs.ucdavis.edu/index.php/research/publications/publicationdetail/?pub_id=1802

Newman, P., \& Jennings, I. (2008). Cities as sustainable ecosystems: Principles and practices. Washington, DC: Island Press.

Niles, J. S. (1994). Beyond telecommuting: A new paradigm for the effect of telecommunications on travel (No. DOE/ER--0626). Washington, DC: USDOE Office of Energy Research, Washington, DC. doi:10.2172/10188598

Nilles, J. M. (1988). Traffic reduction by telecommuting: A status review and selected bibliography. Transportation Research Part A: General, 22(4), 301-317. doi:10.1016/0191-2607(88)90008-8

Nordbakke, S., \& Schwanen, T. (2014). Well-being and mobility: A theoretical framework and literature review focusing on older people. Mobilities, 9(1), 104-129. doi:10.1080/17450101.2013.784542

Norton, P. (2015). Of love affairs and other stories. In S. Zavestoski \& J. Agyeman (Eds.), Incomplete streets: Processes, practices, and possibilities (pp. 17-35). Routledge, New York, NY. doi:10.4324/9781315856537

O'Fallen, C., \& Wallis, I. (2012). A wider look at how travellers value the quality and quantity of travel time (Research Report 469). Wellington, NZ: New Zealand Transport $\begin{array}{lll}\text { Agency. } & \text { Retrieved }\end{array}$ http://www.nzta.govt.nz/assets/resources/research/reports/469/docs/469.pdf

Ohmori, N., \& Harata, N. (2008). How different are activities while commuting by train? A case in Tokyo. Tijdschrift voor economische en sociale geografie, 99(5), 547-561. doi:10.1111/j.1467-9663.2008.00491.x 
Olsson, L. E., Friman, M., Pareigis, J., \& Edvardsson, B. (2012). Measuring service experience: Applying the satisfaction with travel scale in public transport. Journal of Retailing and Consumer Services, 19(4), 413-418. doi:10.1016/j.jretconser.2012.04.002

Olsson, L. E., Gärling, T., Ettema, D., Friman, M., \& Fujii, S. (2013). Happiness and satisfaction with work commute. Social Indicators Research, 111(1), 255-263. doi:10.1007/s11205-012-0003-2

Olsson, L. E., Gärling, T., Fujii, S., Ettema, D., Lekedal, H., \& Friman, M. (2011). Relationship between satisfaction with daily travel and subjective well-being in three urban areas in Sweden. Karlstad, SE: Karlstad University Studies. Retrieved from http://www.diva-portal.org/smash/record.jsf?pid=diva2\%3A409714\&dswid=-3771

Oort, C. J. (1969). The evaluation of travelling time. Journal of Transport Economics and Policy, 3(3), 279-286. Retrieved from http://www.jstor.org/stable/20052155

Oregon Modeling Steering Committee (OMSC). (2011). Oregon Travel and Activity Survey. Salem, OR: Oregon Department of Transportation. Retrieved from http://www.oregon.gov/ODOT/TD/TP/pages/travelsurvey.aspx

Ortúzar, J. d. D., \& Willumsen, L. G. (2011). Modelling transport. Chichester, UK: John Wiley \& Sons, Ltd.

Ory, D. T. (2007). Structural equation modeling of relative desired travel amounts (Unpublished doctoral dissertation). University of California, Davis, CA. Retrieved from https://escholarship.org/uc/item/7rb3 $\times 52 \mathrm{~m}$ 
Ory, D. T., \& Mokhtarian, P. L. (2005). When is getting there half the fun? Modeling the liking for travel. Transportation Research Part A: Policy and Practice, 39(2), 97-123. doi:10.1016/j.tra.2004.09.006

Ory, T. D., \& Mokhtarian, P. L. (2007). Structural equation modeling of desired commute amounts. Presented at the 11th World Congress on Transport Research, Berkeley, CA.

Ory, D. T., \& Mokhtarian, P. L. (2009). Modeling the structural relationships among shortdistance travel amounts, perceptions, affections, and desires. Transportation Research Part A: Policy and Practice, 43(1), 26-43. doi:10.1016/j.tra.2008.06.004

Ory, D. T., Mokhtarian, P. L., Redmond, L. S., Salomon, I., Collantes, G. O., \& Choo, S. (2004). When is commuting desirable to the individual? Growth and Change, 35(3), 334-359. doi:10.1111/j.1468-2257.2004.00252.x

Páez, A., \& Whalen, K. (2010). Enjoyment of commute: A comparison of different transportation modes. Transportation Research Part A: Policy and Practice, 44(7), 537-549. doi:10.1016/j.tra.2010.04.003

Pas, E. I. (1985). State of the art and research opportunities in travel demand: Another perspective. Transportation Research Part A: General, 19(5), 460-464. doi:10.1016/0191-2607(85)90048-2

Pawlak, J., Polak, J. W., \& Sivakumar, A. (2015). Towards a microeconomic framework for modelling the joint choice of activity-travel behaviour and ICT use. Transportation Research Part A: Policy and Practice, 76, 92-112. doi:10.1016/j.tra.2014.10.013

Penedo, F. J., \& Dahn, J. R. (2005). Exercise and well-being: a review of mental and physical health benefits associated with physical activity. Current Opinion in Psychiatry, 18(2), 189-193. doi:10.1097/00001504-200503000-00013 
Pinjari, A., \& Bhat, C. (2006). Nonlinearity of response to level-of-service variables in travel mode choice models. Transportation Research Record: Journal of the Transportation Research Board, 1977, 67-74. doi:10.3141/1977-11

Portland Bureau of Transportation (PBOT). (2017). Parking. Portland, OR: PBOT. Retrieved from https://www.portlandoregon.gov/transportation/34782

Poposki, E. M., \& Oswald, F. L. (2010). The multitasking preference inventory: Toward an improved measure of individual differences in polychronicity. Human Performance, 23(3), 247-264. doi:10.1080/08959285.2010.487843

Pucher, J., Buehler, R., Merom, D., \& Bauman, A. (2011). Walking and cycling in the United States, 2001-2009: Evidence from the National Household Travel Surveys. American Journal of Public Health, 101(S1), S310-S317. doi:10.2105/AJPH.2010.300067

Rails-to-Trails Conservancy (RTC). (2014). Trail Modeling and Assessment Platform (TMAP). Washington, DC: RTC. Retrieved from https://www.railstotrails.org/ourwork/research-and-information/trail-modeling-and-assessment-platform/

Rasouli, S., \& Timmermans, H. (2014a). Judgments of travel experiences, activity envelopes, trip features and multi-tasking: A panel effects regression model specification. Transportation Research Part A: Policy and Practice, 63, 67-75. doi:10.1016/j.tra.2014.02.012

Rasouli, S., \& Timmermans, H. (2014b). Accounting for heterogeneity in travel episode satisfaction using a random parameters panel effects regression model. Procedia Environmental Sciences, 22, 35-42. doi:10.1016/j.proenv.2014.11.004 
Rasouli, S., \& Timmermans, H. J. P. (2014c). Benefits of travel: Needs versus constraints in uncertain environments. In D. F. Ettema, T. Garling, \& M. Friman (Eds.), Handbook of sustainable travel (pp. 33-52). New York, NY: Springer. doi:10.1007/978-94-0077034-8_3

Rasouli, S., \& Timmermans, H. (2014d). Activity-based models of travel demand: promises, progress and prospects. International Journal of Urban Sciences, 18(1), 3160. doi:10.1080/12265934.2013.835118

Reardon, L., \& Abdallah, S. (2013). Well-being and transport: Taking stock and looking forward. Transport Reviews, 33(6), 634-657. doi:10.1080/01441647.2013.837117

Redmond, L. S. (2000). Identifying and analyzing travel-related attitudinal, personality, and lifestyle clusters in the San Francisco Bay Area (Unpublished master's thesis). University of California, Davis, CA. Retrieved from https://itspubs.ucdavis.edu/index.php/research/publications/publicationdetail/?pub_id=415

Redmond, L. S., \& Mokhtarian, P. L. (2001). The positive utility of the commute: Modeling ideal commute time and relative desired commute amount. Transportation, 28(2), 179-205. doi:10.1023/A:1010366321778

Reichman, S. (1976). Travel adjustments and life styles: A behavioral approach. In P. R. Stopher \& A. H. Meyburg (Eds.), Behavioral travel-demand models (pp. 143-152). Lexington, MA: D.C. Heath and Company.

Revelle, W. (2017). psych: Procedures for personality and psychological research (Version 1.7.5) [Software]. Evanston, IL: Northwestern University. Available from https://CRAN.R-project.org/package=psych 
Rhee, K. A., Kim, J. K., Lee, B. J., Kim, S., \& Lee, Y. I. (2013). Analysis of effects of activities while traveling on travelers' sentiment. Transportation Research Record: Journal of the Transportation Research Board, 2383, 27-34. doi:10.3141/2383-04

Richardson, A. (2003). Some evidence of travelers with zero value of time. Transportation Research Record: Journal of the Transportation Research Board, 1854, 107-113. doi:10.3141/1854-12

Rietveld, P., 2002. Rounding of arrival and departure times in travel surveys: An interpretation in terms of scheduled activities. Journal of Transportation and Statistics $5(1), 71-82$.

Rosen, L. D., Whaling, K., Carrier, L. M., Cheever, N. A., \& Rokkum, J. (2013). The media and technology usage and attitudes scale: An empirical investigation. Computers in Human Behavior, 29(6), 2501-2511. doi:10.1016/j.chb.2013.06.006

Rosenfield, A., \& Zhao, J. (2016). Making the commute count: Quality of productive travel time use. Presented at the 56th Annual Conference of the Association of Collegiate Schools of Planning, Portland, OR.

Rosseel, Y. (2012). lavaan: An R Package for Structural Equation Modeling. Journal of Statistical Software, 48(2), 1-36. doi:10.18637/jss.v048.i02

Russell, J. A. (1980). A circumplex model of affect. Journal of Personality and Social Psychology, 39, 1161-1178. doi:10.1037/h0077714

Russell, J. A. (2003). Core affect and the psychological construction of emotion. Psychological Review, 110(1), 145-172. doi:10.1037/0033-295X.110.1.145 
Russell, M. L. (2012). Travel time use on public transport: What passengers do and how it affects their wellbeing (Unpublished doctoral dissertation). University of Otago, Dunedin, NZ. Retrieved from http://hdl.handle.net/10523/2367

Russell, M., \& Mokhtarian, P. (2015). How real is a reported desire to travel for its own sake? Exploring the 'teleportation' concept in travel behaviour research. Transportation, 42(2), 333-345. doi:10.1007/s11116-014-9546-1

Russell, M., Price, R., Signal, L., Stanley, J., Gerring, Z., \& Cumming, J. (2011). What do passengers do during travel time? Structured observations on buses and trains. Journal of Public Transportation, 14(3), 123-146. doi:10.5038/2375-0901.14.3.7

Ryan, R. M., \& Deci, E. L. (2000). Intrinsic and extrinsic motivations: Classic definitions and new directions. Contemporary Educational Psychology, 25(1), 54-67. doi:10.1006/ceps.1999.1020

Ryan, R. M., \& Deci, E. L. (2001). On happiness and human potentials: A review of research on hedonic and eudaimonic well-being. Annual Review of Psychology, 52(1), 141-166. doi:10.1146/annurev.psych.52.1.141

Ryff, C. D. (1989). Happiness is everything, or is it? Explorations on the meaning of psychological well-being. Journal of Personality and Social Psychology, 57(6), 10691081. doi:10.1037/0022-3514.57.6.1069

Sachs, W. (1992). For love of the automobile: Looking back into the history of our desires. Berkeley, CA: University of California Press.

Salomon, I. (1985). Telecommunications and travel: substitution or modified mobility? Journal of Transport Economics and Policy, 19(3), 219-235. Retrieved from http://www.jstor.org/stable/20052753 
Salomon, I. (1986). Telecommunications and travel relationships: A review. Transportation Research Part A: General, 20(3), 223-238. doi:10.1016/01912607(86)90096-8

Salomon, I., \& Mokhtarian, P. L. (1997). Coping with congestion: Understanding the gap between policy assumptions and behavior. Transportation Research Part D: Transport and Environment, 2(2), 107-123. doi:10.1016/S1361-9209(97)00003-5

Salomon, I., \& Mokhtarian, P. L. (1998). What happens when mobility-inclined market segments face accessibility-enhancing policies? Transportation Research Part D: Transport and Environment, 3(3), 129-140. doi:10.1016/S1361-9209(97)00038-2

Sanders, R. L. (2013). Dissecting perceived traffic risk as a barrier to adult bicycling. Presented at the 92nd Annual Meeting of the Transportation Research Board, Washington, DC. Available from https://trid.trb.org/view.aspx?id=1243060

Sarmiento, S. (1998). Household, gender, and travel. In US DOT (Ed.), Women's travel issues: Proceedings from the second national conference, October 1996 (Report No. FHWA-PL-97-024) (pp. 36-52). Washington, DC: US Department of Transportation.

Satorra, A., \& Bentler, P. M. (1994). Corrections to test statistics and standard errors in covariance structure analysis. In A. von Eye \& C. C. Clogg (Eds.), Latent variables analysis: Applications for developmental research (pp. 399-419). Thousand Oaks, CA: Sage Publications.

Schwanen, T., \& Mokhtarian, P. L. (2003). Does dissonance between desired and current residential neighbourhood type affect individual travel behaviour? An empirical assessment from the San Francisco Bay Area. Presented at the European Transport 
$\begin{array}{llll}\text { Conference, } & \text { Strasbourg, } & \text { FR. } & \text { Retrieved }\end{array}$ https://escholarship.org/uc/item/26k8w6xf

Schwanen, T., \& Mokhtarian, P. L. (2005). What if you live in the wrong neighborhood? The impact of residential neighborhood type dissonance on distance traveled. Transportation Research Part D: Transport and Environment, 10(2), 127-151. doi:10.1016/j.trd.2004.11.002

Schneider, R. J. (2013). Theory of routine mode choice decisions: An operational framework to increase sustainable transportation. Transport Policy, 25, 128-137. doi:10.1016/j.tranpol.2012.10.007

Shaya, G. (2004). The flâneur, the badaud, and the making of a mass public in France, circa 1860-1910. The American Historical Review, 109(1), 41-77. doi:10.1086/530151

Sillano, M., \& Ortúzar, J. d. D. (2005). Willingness-to-pay estimation with mixed logit models: Some new evidence. Environment and Planning A, 37(3), 525-550. doi:10.1068/a36137

Singleton, P. A. (2013). A theory of travel decision-making with applications for modeling active travel demand (Unpublished master's thesis). Portland State University, Portland, OR. doi:10.15760/etd.1493

Singleton, P., \& Clifton, K. (2014). Exploring synergy in bicycle and transit use: Empirical evidence at two scales. Transportation Research Record: Journal of the Transportation Research Board, 2417, 92-102. doi:10.3141/2417-10

Singleton, P. A., \& Clifton, K. J. (2015). Exploring the positive utility of travel through theory and simulation. Presented at the 14th International Conference on Travel Behavior Research, Windsor, UK. 
Singleton, P. A., \& Clifton, K. J. (2017). Considering health in US metropolitan long-range transportation plans: A review of guidance statements and performance measures. Transport Policy, 57, 79-89. doi:10.1016/j.tranpol.2017.02.003

Singleton, P. A., \& Goddard, T. (2016). Cycling by choice or necessity? Exploring the gender gap in bicycling in Oregon. Transportation Research Record: Journal of the Transportation Research Board, 2598, 110-118. doi:10.3141/2598-13

Singleton, P., \& Wang, L. (2014). Safety and security in discretionary travel decision making: Focus on active travel mode and destination choice. Transportation Research Record: Journal of the Transportation Research Board, 2430, 47-58. doi:10.3141/2430-06

Singleton, Jr., R. A., \& Straits, B. C. (2005). Approaches to social research (4th ed.). New York, NY: Oxford University Press.

Small, K. A., \& Song, S. (1992). "Wasteful” commuting: A resolution. Journal of Political Economy, 100(4), 888-898. doi:10.1086/261844

Smith, O. B. (2013). Peak of the day or the daily grind: Commuting and subjective wellbeing (Unpublished doctoral dissertation). Portland State University, Portland, OR. doi:10.15760/etd.1026

Smith, O. (2017). Commute well-being differences by mode: Evidence from Portland, Oregon, USA. Journal of Transport \& Health, 4, 246-254. doi:10.1016/j.jth.2016.08.005

Solnit, R. (2001). Wanderlust: A history of walking. New York, NY: Penguin Books. 
Sonnentag, S., Kuttler, I., \& Fritz, C. (2010). Job stressors, emotional exhaustion, and need for recovery: A multi-source study on the benefits of psychological detachment. Journal of Vocational Behavior, 76, 355-365. doi:10.1016/j.jvb.2009.06.005

Steg, L. (2005). Car use: lust and must. Instrumental, symbolic and affective motives for car use. Transportation Research Part A: Policy and Practice, 39(2), 147-162. doi:10.1016/j.tra.2004.07.001

St-Louis, E., Manaugh, K., van Lierop, D., \& El-Geneidy, A. (2014). The happy commuter: A comparison of commuter satisfaction across modes. Transportation Research Part F: Traffic Psychology and Behaviour, 26, 160-170. doi:10.1016/j.trf.2014.07.004

Stone, A. A., \& Schneider, S. (2016). Commuting episodes in the United States: Their correlates with experiential wellbeing from the American Time Use Survey. Transportation Research Part F: Traffic Psychology and Behaviour, 42, 117-124. doi:10.1016/j.trf.2016.07.004

Susilo, Y. O., Abenza, R., Woodcock, A., Liotopoulos, F., Duarte, A., Osmond, J., ..., \& Diana, M. (2017). Findings from measuring door-to-door travellers' travel satisfaction with traditional and smartphone app survey methods in eight European cities. European Journal of Transport and Infrastructure Research, 17(3), 384-410.

Susilo, Y., Lyons, G., Jain, J., \& Atkins, S. (2012). Rail passengers' time use and utility assessment: 2010 findings from Great Britain with multivariate analysis. Transportation Research Record: Journal of the Transportation Research Board, 2323, 99-109. doi:10.3141/2323-12 
Suzuki, H., Fujii, S., Gärling, T., Ettema, D., Olsson, L. E., \& Friman, M. (2014). Rules for aggregated satisfaction with work commutes. Transportation, 41(3), 495-506. doi:10.1007/s11116-013-9484-3

Taniguchi, A., Grääs, C., \& Friman, M. (2014). Satisfaction with travel, goal achievement, and voluntary behavioral change. Transportation Research Part F: Traffic Psychology and Behaviour, 26, 10-17. doi:10.1016/j.trf.2014.06.004

Thomas, G. O., \& Walker, I. (2015). Users of different travel modes differ in journey satisfaction and habit strength but not environmental worldviews: A large-scale survey of drivers, walkers, bicyclists and bus users commuting to a UK university. Transportation Research Part F: Traffic Psychology and Behaviour, 34, 86-93. doi:10.1016/j.trf.2015.07.016

Thompson, E. R. (2007). Development and validation of an internationally reliable shortform of the positive and negative affect schedule (PANAS). Journal of Cross-Cultural Psychology, 38(2), 227-242. doi:10.1177/0022022106297301

Thompson, E. R., \& Phua, F. T. (2012). A brief index of affective job satisfaction. Group \& Organization Management, 37(3), 275-307. doi:10.1177/1059601111434201

Timmermans, H., \& van der Waerden, P. (2008). Synchronicity of activity engagement and travel in time and space: Descriptors and correlates of field observations. Transportation Research Record: Journal of the Transportation Research Board, 2054, 1-9. doi:10.3141/2054-01

Timmermans, H. J., \& Zhang, J. (2009). Modeling household activity travel behavior: Examples of state of the art modeling approaches and research agenda. Transportation Research Part B: Methodological, 43(2), 187-190. doi:10.1016/j.trb.2008.06.004 
Turcotte, M. (2006). Like commuting? Workers' perceptions of their daily commute. Canadian Social Trends, 82, 35-40. Retrieved from http://www.statcan.gc.ca/pub/11008-x/2006004/9516-eng.htm

United Nations General Assembly. (1948). The universal declaration of human rights. New York, NY: United Nations General Assembly. Retrieved from http://www.un.org/Overview/rights.html

US Census Bureau. (2014). How do we know? Working at home is on the rise. Washington, DC: $\quad$ U.S. Census $\quad$ Bureau. Retrieved from http://www.census.gov/library/infographics/home_based_workers.html

US Census Bureau. (2017). American Community Survey (ACS). Washington, DC: US Department of Commerce. Retrieved from https://www.census.gov/programssurveys/acs/

van den Berg, V. A. C., \& Verhoef, E. T. (2015). Robot cars and dynamic bottleneck congestion: The effects on capacity, value of time and preference heterogeneity (Report TI 2015-062/VIII). Amsterdam, NL: Tinbergen Institute. Retrieved from http://papers.tinbergen.nl/15062.pdf

van der Waerden, P. J., Kemperman, A., Timmermans, H., \& van Hulle, R. (2010). The influence of facilities for multitasking on individual's travel decisions in the context of work trips. Presented at the 89th Annual Meeting of the Transportation Research Board, Washington, DC. Available from http://trid.trb.org/view.aspx?id=910535 van der Waerden, P., Timmermans, H., \& van Neerven, R. (2009). Extent, nature, and covariates of multitasking of rail passengers in an urban corridor: A Dutch case study. 
Transportation Research Record: Journal of the Transportation Research Board, 2110, 106-111. doi:10.3141/2110-13

Västfjäll, D., Friman, M., Gärling, T., \& Kleiner, M. (2002). The measurement of core affect: A Swedish self-report measure derived from the affect circumplex. Scandinavian Journal of Psychology, 43(1), 19-31. doi:10.1111/1467-9450.00265

Venables, W. N., \& Ripley, B. D. (2002). Modern applied statistics with S (4th ed.). New York, NY: Springer.

Victoria Transport Policy Institute (VPTI). (2017). Transportation management associations. Victoria, BC: VPTI. Retrieved from https://www.vtpi.org/tdm/tdm44.htm

Vij, A., Carrel, A., \& Walker, J. L. (2013). Incorporating the influence of latent modal preferences on travel mode choice behavior. Transportation Research Part A: Policy and Practice, 54, 164-178. doi:10.1016/j.tra.2013.07.008

Vij, A., \& Walker, J. L. (2016). How, when and why integrated choice and latent variable models are latently useful. Transportation Research Part B: Methodological, 90, 192217. doi:10.1016/j.trb.2016.04.021

Wachs, M., Taylor, B. D., Levine, N., \& Ong, P. (1993). The changing commute: A casestudy of the jobs-housing relationship over time. Urban Studies, 30(10), 1711-1729 doi:10.1080/00420989320081681

Walker, J. L. (2001). Extended discrete choice models: Integrated framework, flexible error structures, and latent variables (Unpublished doctoral dissertation). Massachusetts Institute of Technology, Cambridge, MA. Retrieved from http://hdl.handle.net/1721.1/32704 
Wardman, M. (1998). The value of travel time: A review of British evidence. Journal of Transport Economics and Policy, 32(3), 285-316. Retrieved from http://www.jstor.org/stable/20053775

Waterman, A. S. (1993). Two conceptions of happiness: Contrasts of personal expressiveness (eudaimonia) and hedonic enjoyment. Journal of Personality and Social Psychology, 64(4), 678-691. doi:10.1037/0022-3514.64.4.678

Waterman, A. S., Schwartz, S. J., Zamboanga, B. L., Ravert, R. D., Williams, M. K., Bede Agocha, V., ... \& Brent Donnellan, M. (2010). The Questionnaire for Eudaimonic WellBeing: Psychometric properties, demographic comparisons, and evidence of validity. The Journal of Positive Psychology, 5(1), 41-61. doi:10.1080/17439760903435208

Watson, D., \& Clark, L. A. (1994). The PANAS-X: Manual for the Positive and Negative Affect Schedule - Expanded Form. Iowa City, IA: University of Iowa. Retrieved from http://ir.uiowa.edu/cgi/viewcontent.cgi?article=1011\&context=psychology_pubs

Watson, D., Clark, L. A., \& Tellegen, A. (1988). Development and validation of brief measures of positive and negative affect: The PANAS scales. Journal of Personality and Social Psychology, 54(6), 1063-1070. doi:10.1037/0022-3514.54.6.1063

Watts, L., \& Urry, J. (2008). Moving methods, travelling times. Environment and Planning D: Society and Space, 26(5), 860-874. doi:10.1068/d6707

White, M. J. (1988). Urban commuting journeys are not" wasteful". The Journal of Political Economy, 96(5), 1097-1110. doi:10.1086/261579

Witlox, F. (2007). Evaluating the reliability of reported distance data in urban travel behaviour analysis. Journal of Transport Geography, 15(3), 172-183. doi:10.1016/j.jtrangeo.2006.02.012 
World Health Organization (WHO). (2017). Global physical activity surveillance. Geneva, CH: WHO. Retrieved from http://www.who.int/chp/steps/GPAQ/en/

Ye, R., \& Titheridge, H. (2017). Satisfaction with the commute: The role of travel mode choice, built environment and attitudes. Transportation Research Part D: Transport and Environment, 53(B), 535-547. doi:10.1016/j.trd.2016.06.011

Yosritzal. (2014). An investigation into the role of technology in influencing the perception and value of travel time by rail (Unpublished doctoral dissertation). Newcastle University, Newcastle, UK. Retrieved from http://hdl.handle.net/10443/2493

Young, W., \& Morris, J. (1981). Evaluation by individuals of their travel time to work. Transportation Research Record, 794, 51-59. Retrieved from http://trid.trb.org/view.aspx?id=172650

Zhang, J., \& Timmermans, H. (2010). Scobit-based panel analysis of multitasking behavior of public transport users. Transportation Research Record: Journal of the Transportation Research Board, 2157, 46-53. doi:10.3141/2157-06

Zhao, F., Pereira, F. C., Ball, R., Kim, Y., Han, Y., Zegras, C., \& Ben-Akiva, M. (2015). Exploratory analysis of a smartphone-based travel survey in Singapore. Transportation Research Record: Journal of the Transportation Research Board, 2494, 45-56. doi:10.3141/2494-06

Zhao, J., \& Lee, J. (2013). Experience or memory: Happiness of commuting. Presented at the 92nd Annual Meeting of the Transportation Research Board, Washington, DC.

Zhao, Z., \& Zhao, J. (2015). Car pride: Psychological structure and behavioral implications. Presented at the 94th Annual Meeting of the Transportation Research Board, Washington, DC. 
Zhu, P. (2012). Are telecommuting and personal travel complements or substitutes? The Annals of Regional Science, 48(2), 619-639. doi:10.1007/s00168-011-0460-6

Zmud, J., Sener, I. N., \& Wagner, J. (2016). Revolutionizing our roadways: Consumer acceptance and travel behavior impacts of automated vehicles. College Station: TX: Texas A\&M Transportation Institute. Retrieved from tti.tamu.edu/documents/TTI2016-8.pdf 
Appendix Survey

Questionnaire survey instrument (Parts I and II)

Commuting Survey 2016

QSTART

You are being invited to participate in a Portland State University research study about your commuting experiences. The information you provide will be analyzed to better understand transportation and commuting behaviors.

This study is being conducted by Patrick Singleton and Dr. Kelly Clifton, from the Department of Civil \& Environmental Engineering at Portland State University. The research is part of a doctoral dissertation, with funding from the National Institute for Transportation and Communities, a program of the Transportation Research and Education Center for Portland State University.

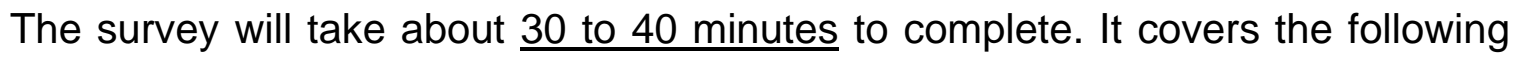
topics:

- Your personal and transportation characteristics

- Your home, your job, and your typical commute

- Your most recent commute trip, including things you did and things you felt and experienced while commuting

There is an optional Part II survey that will take about 15 to 20 minutes to complete. It covers the following topics:

- How you get around using different means of transportation

- Your thoughts about various topics, including multitasking, satisfaction, and attitudes

- Your physical activity levels

There are no known risks if you decide to participate in this research study. There are no costs to you for participating in the study. There are no direct benefits to you for participating in the study. However, the information learned in this study may provide more general benefits to society, such as increased insight into how and why people commute in different ways.

If you complete the survey, you will have the opportunity to enter a drawing to win one of ten $\$ 100$ Visa gift cards. If you also complete the optional Part II survey, you will receive a second entry into the drawing. If you wish to enter the drawing, you will be asked to provide an email address, which will be deleted after prizes are awarded. 
If you have any questions about the study, please email tstudy@pdx.edu, call 503893-9677, or write to: Patrick Singleton \& Dr. Kelly Clifton, Portland State University, Civil \& Environmental Engineering, PO Box 751 - CEE, Portland, OR 97207-0751. The Portland State University Institutional Review Board has reviewed this project. If you have any concerns about your rights in this study, please contact the PSU Office of Research Integrity at 503-725-2227 or email hsrrc@pdx.edu.

This survey is anonymous, although you may be asked to provide some indirectlyidentifying information (demographics, home and job location). This personal information will be treated confidentially. No one will attempt to identify you or your answers, and no one will know whether or not you participated in the study. Your employer will not have access to your responses. Individuals from the Institutional Review Board may inspect these records. Should the data be published, no individual information will be disclosed.

Your participation in this study is voluntary. You may decline to answer a particular question by selecting "Prefer not to answer" or skipping the question. You may exit the survey at any time by closing the survey window or tab. By starting this survey, you are voluntarily agreeing to participate.

By clicking "Accept" at the end of this page, you are consenting to participate in this survey. If you do not consent, please click "Decline" to navigate away from the survey.

\section{CONSENT}

Please indicate your consent to participate in this survey.

O Accept

O Decline 


\title{
ELIG1
}

Welcome to this survey! Please answer the following questions to confirm your eligibility.

\author{
Are you 18 years of age or older? \\ O Yes \\ O No
}

ELIG2

Do you have a job outside the home to which you commute at least once a week? This includes any volunteer work you do on a regular basis.

O Yes

O No

\section{QINTRO}

Thank you! Before we begin, please note the following.

Your responses are optional. If you prefer not to answer a particular question, you may skip it.

If you want to change your answer to a previous question, you may go back by clicking the Back button on the bottom left of each page. Do not use your browser's back button.

The survey is split into several sections, each with an estimated completion time. 
QA

To start, we would like to ask you some basic information about your:

- Personal characteristics

- Transportation characteristics

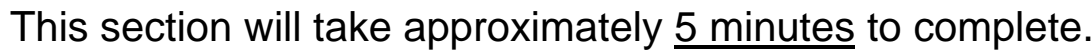

AGE

First, the following demographic questions ask about you and your household. This information will be used to classify your responses and to make sure this survey reaches a broad population.

What is your age?

O 18-24

O 25-34

O $35-44$

O $45-54$

O 55-64

O $65-74$

O 75-84

O $85+$

RACE

Which of the following describe you? (Check all that apply.)

White

- Hispanic, Latino, or Spanish origin

Black or African American

Asian

- American Indian or Alaska Native

- Middle Eastern or North African

- Native Hawaiian or Other Pacific Islander

$\square$ Other please specify) 
GEND

How do you describe yourself?

O Female

O Male

O Transgender

O Do not identify as female, male, or transgender

EDUC

What is the highest degree or level of school you have completed?

O Not a high school graduate, grade 12 or less

O High school graduate (diploma or GED)

O Some college credit but no degree

O Associate or technical school degree

O Bachelor's or undergraduate degree

O Graduate or professional degree

O Other (please specify)

STUD

Are you currently enrolled in any type of school?

O Yes, full-time

O Yes, part-time

O No

HHSIZE

Including yourself, how many people live in your home?

O 1 (just me)

O 2

O 3

○ 4

O 5

O 6

O 7

O 8+ 
HHMEM

In this question, you are Person 1. For each other person who lives in your home, indicate if they are: (Check all that apply.)

\begin{tabular}{|l|c|c|c|c|c|c|c|}
\hline & Person 2 & 3 & 4 & 5 & 6 & 7 & 8 \\
\hline Related to you & $\square$ & $\square$ & $\square$ & $\square$ & $\square$ & $\square$ & $\square$ \\
Your spouse or partner & $\square$ & $\square$ & $\square$ & $\square$ & $\square$ & $\square$ & $\square$ \\
Employed & $\square$ & $\square$ & $\square$ & $\square$ & $\square$ & $\square$ & $\square$ \\
A student & $\square$ & $\square$ & $\square$ & $\square$ & $\square$ & $\square$ & $\square$ \\
16 years old or younger & $\square$ & $\square$ & $\square$ & $\square$ & $\square$ & $\square$ & $\square$ \\
65 years old or older & $\square$ & $\square$ & $\square$ & $\square$ & $\square$ & $\square$ & $\square$ \\
\hline
\end{tabular}

HHINC

Including yourself and the people you share finances with, what is your approximate total annual income (before taxes)?

O $\$ 0-\$ 14,999$

O $\$ 15,000-\$ 24,999$

O $\$ 25,000-\$ 34,999$

O $\$ 35,000-\$ 49,999$

O $\$ 50,000-\$ 74,999$

O $\$ 75,000-\$ 99,999$

O $\$ 100,000-\$ 149,999$

O $\$ 150,000+$

○ Don't know 
DISAB

Next are questions about you and your household's transportation-related characteristics.

Do you have a physical condition that seriously limits or prevents you from doing any of the following? (Check all that apply, or None.)

$\square$ Seeing

Hearing

S Sitting

Standing

Climbing stairs

W Walking

Riding a bicycle

D Driving an automobile

- Riding in an automobile

Using public transit

Other (please specify)

口 None

SKILL

Do you know how to:

\begin{tabular}{|l|c|c|c|}
\hline & Yes & Not well & No \\
\hline Ride a bicycle & 0 & 0 & 0 \\
Drive an automobile & 0 & 0 & 0 \\
Use public transit & 0 & 0 & 0 \\
\hline
\end{tabular}

DLIC

Do you have a driver's license?

O Yes

O No 
HHVEH

How many of the following are available to you at your home? Only count those in working condition that are privately owned or leased by you or people you live with.

\begin{tabular}{|l|c|c|c|c|c|c|c|c|c|}
\hline $\begin{array}{l}\text { Bicycles } \\
\begin{array}{l}\text { Automobiles (cars, trucks, } \\
\text { vans, and SUVs) }\end{array}\end{array}$ & 0 & 1 & 2 & 3 & 4 & 5 & 6 & 7 & $8+$ \\
$\begin{array}{l}\text { Other vehicles (please } \\
\text { specify) }\end{array}$ & 0 & 0 & 0 & 0 & 0 & 0 & 0 & 0 & 0 \\
\hline
\end{tabular}

\section{TPASS}

Do you have any of the following public transit passes? (Check all that apply.)

The pass could be through your employer.

TriMet annual, monthly, or 30-day pass

TriMet 14-day or 7-day pass

- Portland Streetcar annual or monthly pass

C-TRAN annual or monthly pass

C-TRAN 10-ride punch card

Other (please specify)

TPASSEMP

Did you get this transit pass through your employer?
$\mathrm{O}$ Yes
O No
O Don't know

VSHMEM

Do you belong to any of the following car- and bike-sharing services? (Check all that apply.)

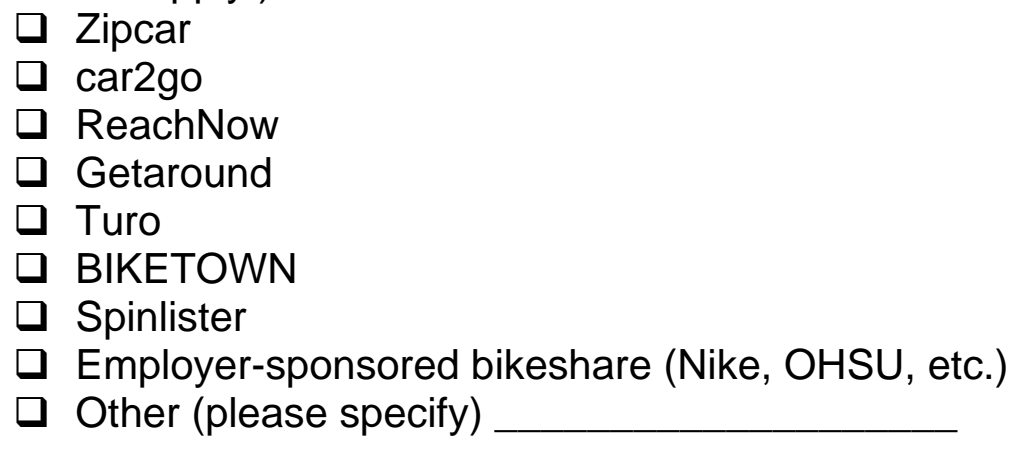




\section{TSERV}

Within the past year, have you used any of the following in the Portland area for any reason? (Check all that apply.)

TriMet bus

C-TRAN bus

Portland Streetcar

MAX light rail

WES commuter rail

P Portland Aerial Tram

BIKETOWN

$\square$ Uber

Lyft

T Taxi

Zipcar

car2go

$\square$ Rental car

\section{MFREQ}

Within the past week, have you used any of the following means or modes of transportation to get around the Portland area? (Check all that apply.) Think about all the times you left your home for any reason, whether to visit a neighbor, go for a walk, or shop across town. Consider even short trips, like going around the block or getting to/from public transit.

Walking

Bicycling

- Automobile, driver (personal car/truck/van/SUV, shared vehicle, Zipcar, car2go, etc.)

- Automobile, passenger (personal vehicle, carpool, taxi, Uber, Lyft, etc.)

Public transit (bus, streetcar, light rail, etc.)

Other (please specify) 
QB

Now, we would like to ask you some questions about commuting, including:

- Your home

- Your job

- How you typically commute between the two Your thoughts about your commute and about commuting scenarios

This section will take approximately 10 minutes to complete.

HTYPE

First are questions about your home or place of residence.

Which best describes your home?

O Mobile home or trailer

S Single-family house, detached from any other house

O Single-family house, attached to other houses (row house)

O Duplex / building with 2 apartments/condos

O Building with 3-19 apartments/condos

O Building with 20+ apartments/condos

O Other (please specify)

HTEN

Do you own or rent your home?

O Owned or mortgaged

O Rented

HDUR

How long have you lived in your home?

O $0-1$ year

O 1-2 years

O 2-5 years

O $5-10$ years

O $10+$ years

HLOC

Where is your home located? Please enter the nearest major intersection and the ZIP code. For example: SW Main St. \& SW 1st Ave., 97204. Alternatively, you may enter a neighborhood or nearby landmark, such as a park or school. 
WOCC

Next, the following questions ask about your job, work, or place of employment. If you have more than one job, think about the one you do most often or for the most hours.

What is your occupation?

WSELF

Are you self-employed?

O Yes

O No

O Don't know

\section{WDAYS}

In an average week, how many days do you: Commute to your job Work from home

WHRS

In an average week, how many hours do you work, total? Hours per week

WFLEX

How flexible is your work schedule?

$O$ Very inflexible

O Somewhat inflexible

O Neither flexible nor inflexible

O Somewhat flexible

O Very flexible

WLOC

Where is your job located? If you work in more than one location, consider the place you go to most often or for the most hours. Please enter the nearest major intersection and (if you know it) the ZIP code. For example: SW Main St. \& SW 1st Ave., 97204. Alternatively, you may enter a neighborhood or nearby landmark, such as a park or school. 


\section{TYPMODE}

Next, the following questions ask about how you typically travel between home and work at this time of year. Base your answers about a "typical commute" on your experiences during a normal or average day. If you do not have a typical commute, think about the way you commute most often.

What transportation mode do you use for your typical commute? If you use more than one mode to get to/from work on a normal day, select the one used for the longest duration.

O Walking

O Bicycling

O Automobile, driver (personal car/truck/van/SUV, shared vehicle, Zipcar, car2go, etc.)

O Automobile, passenger (personal vehicle, carpool, taxi, Uber, Lyft, etc.)

Public transit (bus, streetcar, light rail, etc.)

Other (please specify)

TYPMODEAUTO

What kind of automobile is that?

O Personal vehicle

O Commercial vehicle

O Carpool or vanpool vehicle

O Uber

O Lyft

O Taxi

O Zipcar

O car2go

O Rental car

Other (please specify)

\section{TYPMODETRAN}

What kind of public transit vehicle is that? (Check all that apply.)

$\square$ TriMet bus

C-TRAN bus

- Portland Streetcar

MAX light rail

WES commuter rail

- Portland Aerial Tram

$\square$ Other (please specify) 


\section{TYPDIST}

In miles, about how far is your typical one-way commute? Please be as precise as possible. For example: 3.9.

\section{Distance (miles)}

Between home and work

\section{TYPDUR}

In minutes, about how long is your typical one-way commute in each direction? Please be as precise as possible. For example: 32 .

\begin{tabular}{|l|l|}
\hline From home to work & Duration (minutes) \\
\hline From work to home & \\
\hline
\end{tabular}

\section{QB4T0}

Next, the following are six hypothetical questions about transportation and commuting.

Consider your normal work responsibilities and your typical commute. Each of the following questions present you with two options in which the time you must spend working (work time) and/or the time you must spend commuting (commute travel time) either increases (+) or decreases (-) by 10 minutes/day, compared to your normal work responsibilities and your typical commute. Your pay and your travel costs do not change.

Please select the option you would prefer, even if only slightly. Some choices may be obvious. 
VTTS1

Which would you prefer?

O Work time increases (+) by 10 minutes/day with no additional pay

O Work time decreases ( $(-)$ by 10 minutes/day with no loss in pay

VTTS2

Which would you prefer?

O Commute travel time increases (+) by 10 minutes/day with no additional cost

O Commute travel time decreases (-) by 10 minutes/day with no reduction in cost

VTTS3

Which would you prefer?

O Work time increases (+) by 10 minutes/day with no additional pay

O Commute travel time increases (+) by 10 minutes/day with no additional cost

VTTS4

Which would you prefer?

O Work time decreases (-) by 10 minutes/day with no loss in pay

O Commute travel time decreases (-) by 10 minutes/day with no reduction in cost

VTTS5

Which would you prefer?

O Work time increases (+) by 10 minutes/day with no additional pay

O Commute travel time decreases (-) by 10 minutes/day with no reduction in cost

VTTS6

Which would you prefer?

O Work time decreases (-) by 10 minutes/day with no loss in pay

O Commute travel time increases (+) by 10 minutes/day with no additional cost 


\section{QB4T1}

In responding to the next few questions, think carefully about the things you like and dislike about commuting.

For instance, people may like certain aspects of commuting:

- The opportunity for transitioning between roles (between work and home, etc.)

- Time for yourself (for thinking, relaxing, etc.)

- Time to do certain things (listen to music, communicate with others, etc.)

- Enjoyment of the environment

- Exercise

On the other hand, some people dislike other aspects of commuting:

- The time it takes away from other things

- Congestion

- Stress

- Exposure to weather

- Being crowded by strangers

With these pros and cons in mind, please answer the following questions.

\section{TYPSAT}

Taking all things together, in general, how satisfied are you with your typical commute?

V Very dissatisfied

O Somewhat dissatisfied

O Neither satisfied nor dissatisfied

O Somewhat satisfied

O Very satisfied

\section{TYPSATTT}

How satisfied are you with your typical one-way commute travel times?

O Very dissatisfied

O Somewhat dissatisfied

O Neither satisfied nor dissatisfied

O Somewhat satisfied

O Very satisfied 


\section{TTSAT}

How satisfied would you be with the following one-way commute travel times?

Very dissatisfied (1), Somewhat dissatisfied (2), Neither satisfied nor dissatisfied (3), Somewhat satisfied (4), Very satisfied (5)

\begin{tabular}{|l|l|l|l|l|l|}
\hline 0 minutes & $(1)$ & $(2)$ & $(3)$ & $(4)$ & $(5)$ \\
10 minutes & 0 & 0 & 0 & 0 & 0 \\
20 minutes & 0 & 0 & 0 & 0 & 0 \\
30 minutes & 0 & 0 & 0 & 0 & 0 \\
45 minutes & 0 & 0 & 0 & 0 & 0 \\
1 hour & 0 & 0 & 0 & 0 & 0 \\
$2+$ hours & 0 & 0 & 0 & 0 & 0 \\
\hline
\end{tabular}

\section{TTIDEAL}

Suppose that you could live as close to work (or work as close to home) as you want to, and use any transportation mode. For example: You could live where you work and have a 0 minute commute.

For you, what would be your ideal one-way commute travel time?

\section{Duration (minutes)}

Ideal travel time 


\title{
TPORT
}

Now, suppose you could snap your fingers or blink your eyes and be instantly transported or "teleported" between home and work. Further, suppose that the cost of teleporting is not an issue, and that teleporting is $100 \%$ safe.

Would you teleport, or would you prefer to spend some time commuting?

O I would rather teleport

O I would rather spend some time commuting

\section{Answer If "I would rather teleport" Is Selected}

\section{TPORTY}

Why? (Check all that apply.)

My commute is a waste of time.

I dislike most aspects of my commute.

I am always in a rush.

I I have other things to do.

I like trying new technology.

$\square$ Other (please specify)

\author{
Answer If "I would rather spend some time commuting" Is Selected \\ TPORTN \\ Why? (Check all that apply.) \\ 口 I like to use my commute time productively. \\ I enjoy certain aspects of my commute. \\ I I would be uneasy or afraid to teleport. \\ - Teleportation is and always will be impossible. \\ Other (please specify)
}


QC

Thank you for your responses! You're about $45-50 \%$ done.

Now, we would like to ask you some detailed questions about your most recent commute trip from home to work, including:

Basic trip information

Things you did while commuting

Things you felt and experienced while commuting

Your expectations

This section will take approximately 10 to 15 minutes to complete.

CDAY1

First, the following questions ask for basic information about your most recent commute trip from home to work.

When did this commute trip take place?

O Today

Y Yesterday

O 2 days ago

O 3 days ago

4+ days ago

CDAY2

Which day of the week was that?

O Monday

O Tuesday

O Wednesday

O Thursday

O Friday

O Saturday

O Sunday

\section{CTIME}

When did you leave home, and when did you arrive at work? Please be as precise as possible. For example: 8:32 AM. Leave home Arrive at work 
CMODE

What transportation mode did you use for this commute trip? If you used more than one mode, please select the one used for the longest duration.

O Walking

O Bicycling

O Automobile, driver (personal car/truck/van/SUV, shared vehicle, Zipcar, car2go, etc.)

O Automobile, passenger (personal vehicle, carpool, taxi, Uber, Lyft, etc.)

O Public transit (bus, streetcar, light rail, etc.)

Other (please specify)

\section{Answer If CMODE different from TYPMODE} CMODETYPWHY

This is different from the typical commute mode you selected. Why?

\section{CMODEAUTO}

What kind of automobile was that? (Check all that apply.)

a Personal vehicle

- Commercial vehicle

Carpool or vanpool vehicle

U Uber

$\square$ Lyft

Taxi

Zipcar

car2go

Rental car

Other (please specify)

\section{CPARK1}

Where did you park?

O On-street parking

O Parking lot

O Parking garage

Other (please specify)

\section{CPARK2}

Where was this parking located? Please enter the nearest major intersection or name of the parking facility. 


\section{CMODETRAN}

What kind of public transit vehicle was that? (Check all that apply.)

TriMet bus

C-TRAN bus

Portland Streetcar

MAX light rail

$\square$ WES commuter rail

口 Portland Aerial Tram

O Other (please specify)

\section{CMODEOTH}

Did you use any other transportation modes on this commute trip? (Check all that apply, or No other modes.) Include modes used to get to and from public transit, or to get from a parking space to your workplace.

$\square$ Walking (1)

Bicycling (2)

- Automobile, driver (personal car/truck/van/SUV, shared vehicle, Zipcar, car2go, etc.) (3)

- Automobile, passenger (personal vehicle, carpool, taxi, Uber, Lyft, etc.) (4)

Public transit (bus, streetcar, light rail, etc.) (5)

Other (please specify) (90)

No other modes (99)

\section{CCOST}

Approximately how much did it cost to make this commute trip? Please be as precise as possible. For example: 4.75 .

Include the following (as appropriate):

- Parking costs

- Public transit fares

- Taxi, Uber, or Lyft fares

- Any other direct costs you paid on that day

Do not include indirect costs for things like gas, maintenance, or depreciation. If you pay monthly or annually for parking or public transit, do not include those costs. If you did not pay anything, put 0 .

Cost $(\$)$ 
CPEOP

How many other people were you traveling with? Only count people you know.

For example: don't count other passengers on the bus.

O 0 (just me)

O 1

○ 2

O 3

O 4

O $5+$

\section{CNUMSTOP}

Did you stop anywhere on your way to work? If so, how many places did you visit? Don't count places where you only changed modes, such as a bus stop or parking space.

O 0 (none)

O 1

O 2

O $3+$

C1LOC

Where was your first stop? Please enter the nearest major intersection.

C2LOC

Where was your second stop?

C3LOC

Where was your third stop? 
CSTOPPURP

What did you do there? (Check all that apply.)

\begin{tabular}{|l|c|c|c|} 
& First & Second & Third \\
Work & stop & stop & stop \\
School & $\square$ & $\square$ & $\square$ \\
Grocery shopping & $\square$ & $\square$ & $\square$ \\
Other shopping & $\square$ & $\square$ & $\square$ \\
Household errands, personal business, and health & $\square$ & $\square$ & $\square$ \\
care (bank, cleaners, doctor, dentist, etc.) & $\square$ & $\square$ & $\square$ \\
Civic and religious activities & $\square$ & $\square$ & $\square$ \\
Eating or drinking outside of home (restaurant, bar, & $\square$ & $\square$ & $\square$ \\
etc.) & $\square$ & $\square$ & $\square$ \\
Taking other people places (to school, etc.) & $\square$ \\
Indoor entertainment and recreation (movie, museum, & $\square$ & $\square$ & $\square$ \\
gym, indoor concert, etc.) & & & \\
Outdoor entertainment and recreation, in one location & $\square$ & $\square$ & $\square$ \\
(sports, other athletics, outdoor festival, etc.) & & & \\
Exercise as transportation, returning to your starting & $\square$ & $\square$ & $\square$ \\
location (going for a walk, jogging, recreational & $\square$ & & \\
bicycling, etc.) & $\square$ & $\square$ & $\square$ \\
Visiting friends and family & $\square$ & $\square$ & $\square$ \\
Other (please specify) & $\square$ & $\square$ & $\square$ \\
Other (please specify) & $\square$ & $\square$ & $\square$ \\
Other (please specify) & & $\square$
\end{tabular}

CSTOPDUR

How long did you stay there? Please be as precise as possible. For example: 32. 
Loop based off question CMODE (Selected Choice)

\{Loop \#, Field 1, Field 2, Field 3, Field 4, Field 5\}

Loop 1: Walking, walking, walked, commuted by walking, Walking

Loop 2: Bicycling, bicycling, rode a bicycle, commuting by bicycle, Bicycling

Loop 3: Automobile, driver (personal car/truck/van/SUV, shared vehicle, Zipcar, car2go, etc.), driving an automobile, drove an automobile, commuted by automobile as a driver, Driving an automobile

Loop 4: Automobile, passenger (personal vehicle, carpool, taxi, Uber, Lyft, etc.), riding as a passenger in an automobile, rode as a passenger in an automobile, commuting by automobile as a passenger, Riding as a passenger in an automobile

Loop 5: Public transit (bus, streetcar, light rail, etc.), riding public transit, rode public transit, commuting by public transit, Riding public transit

Loop 6: Other, using some other mode, used some other mode, commuting by some other mode, Using some other mode

QC2TWALK, QC2TBIKE, QC2TAUTO, QC2TTRAN, QC2TOTH

Now, we would like to ask you some detailed questions about the things you did and the things you felt and experienced while $\$\{I m: / / F i e l d / 2\}$ on your most recent commute to work. 


\section{CTAPART}

The following questions ask about the things you did while $\$\{/ m: / / F i e l d / 2\}$ on your most recent commute to work. Think about everything you did after leaving your home until arriving at work. For instance, consider what you did while on board, getting to/from, and waiting for public transit.

While $\$\{$ Im://Field/2\}, did you do any of the following things? (Check all that apply, or Doing nothing.)

- Talking face-to-face with people you know

Talking face-to-face with strangers

Talking on the phone

Texting, emailing, or other messaging

Reading print (newspaper, book, etc.)

Reading electronically (e-book, website, etc.)

Writing or editing paper documents

Writing or editing electronic documents

Listening to music, radio, or other audio

Watching movie, TV, or other video

U Using social websites or apps (Facebook, Twitter, Linkedln, Tumblr, Instagram, etc.)

Playing game (Pokémon Go, puzzle, etc.)

Eating food; drinking beverage

Smoking or vaping

- Personal grooming (shaving, makeup, etc.)

Caring for children or pets (dog walking, etc.)

Singing; dancing

Exercising or being physically active

- Planning or navigating this trip

- Viewing scenery; watching people

Thinking or daydreaming

$\square$ Sleeping or snoozing

Doing nothing

Other (please specify)

For each selected in CTAPART

CTADUR

While $\$\{\mathrm{Im}: / /$ Field/2\}, approximately what percentage of your commute travel time did you spend doing these things? Your totals may add up to more than $100 \%$ if you did two or more things at once.

Percentage (\%): 0, 10, 20, 30, 40, 50, 60, 70, 80, 90, 100, Don't know 


\section{CTAUSE}

In terms of its value to you, overall, how useful would you rate the time you spent $\$\{\mathbf{I m}: / /$ Field/2\}? Ignore the value of getting to your destination, and think only about the things you did while $\$\{\mathrm{Im}: / /$ Field $/ 2\}$ and the time you spent doing them.

O Mostly wasted

O Somewhat wasted

O Neither wasted nor useful

O Somewhat useful

O Mostly useful

\section{CTEPNA}

Next, the following questions ask about your feelings and experiences while $\$\{I m: / / F i e l d / 2\}$ on your most recent commute to work. Think about everything you felt or experienced after leaving your home until arriving at work. For instance, consider your experience while on board, getting to/from, and waiting for public transit.

Thinking about yourself and your most recent commute to work, indicate to what extent you felt the following while $\$\{\mathbf{I m : / / F i e l d / 2 \}}$.

Very slightly or not at all (1), A little (2), Moderately (3), Quite a bit (4), Extremely (5)

\begin{tabular}{|l|c|c|c|c|c|}
\hline Upset & $(1)$ & $(2)$ & $(3)$ & $(4)$ & $(5)$ \\
Hostile & 0 & 0 & 0 & 0 & 0 \\
Alert & 0 & 0 & 0 & 0 & 0 \\
Ashamed & 0 & 0 & 0 & 0 & 0 \\
Inspired & 0 & 0 & 0 & 0 & 0 \\
Nervous & 0 & 0 & 0 & 0 & 0 \\
Determined & 0 & 0 & 0 & 0 & 0 \\
Attentive & 0 & 0 & 0 & 0 & 0 \\
Afraid & 0 & 0 & 0 & 0 & 0 \\
Active & 0 & 0 & 0 & 0 & 0 \\
\hline
\end{tabular}


CTEAFF

Indicate to what extent you felt the following while $\$\{\operatorname{Im}: / /$ Field $/ 2\}$.

\begin{tabular}{|l|c|c|c|c|c|}
\hline Excited & $(1)$ & $(2)$ & $(3)$ & $(4)$ & $(5)$ \\
Strong & 0 & 0 & 0 & 0 & 0 \\
Vulnerable & 0 & 0 & 0 & 0 & 0 \\
Proud & 0 & 0 & 0 & 0 & 0 \\
Angry & 0 & 0 & 0 & 0 & 0 \\
Bold & 0 & 0 & 0 & 0 & 0 \\
Frustrated & 0 & 0 & 0 & 0 & 0 \\
Timid & 0 & 0 & 0 & 0 & 0 \\
Calm & 0 & 0 & 0 & 0 & 0 \\
Stressed & 0 & 0 & 0 & 0 & 0 \\
\hline
\end{tabular}

CTESEN

Did you feel any of the following, at least a little, while $\$\{I \mathrm{Im}: / /$ Field $/ 2\}$ ? (Check all that apply, or None of the above.)

․ Hot

Cold

Wet

$\square$ Sore

Dirty

Sweaty

None of the above 


\section{CTESTS}

For each of the following pairs, select the choice that best corresponds to your overall experience $\$\{\mathbf{I m}: / /$ Field $/ \mathbf{2}\}$ on your most recent commute to work. For example: If you were very tense, select the leftmost choice. If you were very relaxed, select the rightmost choice. If you were neither tense nor relaxed, select the middle or neutral choice.

\begin{tabular}{|c|c|c|c|c|c|c|c|c|}
\hline & $\leftarrow$ & & & neutral & & & $\rightarrow$ & \\
\hline I was very tense. & 0 & 0 & 0 & 0 & 0 & 0 & O & I was very relaxed. \\
\hline I was very bored. & O & 0 & 0 & 0 & 0 & 0 & $\mathrm{O}$ & $\begin{array}{l}\text { I was very } \\
\text { enthusiastic. }\end{array}$ \\
\hline I was very sad. & O & O & $\mathrm{O}$ & $\mathrm{O}$ & O & 0 & $\mathrm{O}$ & I was very happy. \\
\hline I was very tired. & 0 & 0 & 0 & O & 0 & 0 & $\mathrm{O}$ & $\begin{array}{l}\text { I was very } \\
\text { energized. }\end{array}$ \\
\hline $\begin{array}{l}\text { I was very } \\
\text { distressed. }\end{array}$ & 0 & 0 & 0 & 0 & 0 & 0 & 0 & $\begin{array}{l}\text { I was very } \\
\text { content. }\end{array}$ \\
\hline $\begin{array}{l}\text { My trip went } \\
\text { poorly. }\end{array}$ & 0 & 0 & 0 & 0 & 0 & 0 & 0 & $\begin{array}{l}\text { My trip went } \\
\text { smoothly. }\end{array}$ \\
\hline $\begin{array}{l}\text { My trip was } \\
\text { displeasing. }\end{array}$ & O & O & O & O & O & O & O & $\begin{array}{l}\text { My trip was } \\
\text { enjoyable. }\end{array}$ \\
\hline $\begin{array}{l}\text { I was worried I } \\
\text { wouldn't arrive on } \\
\text { time. }\end{array}$ & 0 & 0 & O & O & 0 & 0 & $\mathrm{O}$ & $\begin{array}{l}\text { I was confident I } \\
\text { would arrive on } \\
\text { time. }\end{array}$ \\
\hline $\begin{array}{l}\text { My trip was the } \\
\text { worst I can } \\
\text { imagine. }\end{array}$ & O & $\mathrm{O}$ & O & $\mathrm{O}$ & 0 & O & $\mathrm{O}$ & $\begin{array}{l}\text { My trip was the } \\
\text { best I can imagine. }\end{array}$ \\
\hline
\end{tabular}




\section{CTEDES}

For the following questions: check all that apply, or select "None of the above".

Thinking about your most recent commute to work, did $\$\{I m: / / F i e l d / 2\}$ allow you, at least a little, to fulfill your desire for:

$\square$ Variety

Control

adventure

Companionship

- Freedom

P Privacy

$\square$ Safety

a Comfort

Stress relief

A routine

A challenge

A buffer between home and work

Membership in a group or class

None of the above

\section{CTEEXP}

Did $\$\{\operatorname{lm}: / /$ Field/2\} allow you, at least a little, to express your:

$\square$ Independence

Social status

$\square$ Self-identity

Courage

M Mastery of a skill

Environmental values

None of the above

CTEIMP

Did $\$\{1 \mathrm{~m}: / /$ Field/2\} allow you, at least a little, to improve your:

$\square$ Self-confidence

Mental health

Physical health

․ None of the above 


\section{CTELIKE}

Overall, how much did you like $\$\{\mid \mathrm{Im}: / /$ Field/2\} on your most recent commute to work?

O Strongly disliked

O Somewhat disliked

O Neither liked nor disliked

O Somewhat liked

O Strongly liked

CEXP

Now, think back to when you decided to make this commute trip, and your expectations of it.

Overall, how well did this commute trip match your expectations?

O Much worse than expected

O Somewhat worse than expected

O About the same as expected

O Somewhat better than expected

Ouch better than expected

\section{CCHNG}

Knowing what you know now, would you change any of the following decisions related to this commute trip? (Check all that apply, or Nothing.)

C Choose a different transportation mode

T Take a different route

Leave at a different time

Other (please specify)

Nothing; I would make the same decisions

CEXPTEXT

Was there anything special or different about this commute trip? Did anything unexpected happen? 
QD

Thanks for your responses! You're about $70-75 \%$ done.

Now, we would like to ask you some further questions about your most recent commute trip from home to work, including:

- Other modes of transportation that you could have used

- Things you would have done while commuting using other modes

- Things you would have felt and experienced while commuting using other modes

This final section will take approximately $\underline{5 \text { to } 15 \text { minutes }}$ to complete.

\section{COHOME}

The mode you used for your most recent commute trip to work was: \$\{q://QID170/ChoiceGroup/SelectedChoicesTextEntry\}. If this mode was not available, what would you have done?

O I would have commuted using a different mode.

O Instead of commuting, I would have worked from home.

Other (please specify)

\section{COMODE}

Which other modes did you consider using for your most recent commute to work? Please select at least one other mode, but select all that you considered using.

If you would have worked from home, select the mode you would have used if you had to commute to work for some reason.

Walking (1)

Bicycling (2)

- Automobile, driver (personal car/truck/van/SUV, shared vehicle, Zipcar, car2go, etc.) (3)

Automobile, passenger (personal vehicle, carpool, taxi, Uber, Lyft, etc.) (4)

Public transit (bus, streetcar, light rail, etc.) (5)

$\square$ Other (please specify) (6) 
For each selected in COMODE COMODERANK

How would you rank them? Put the most likely mode 1st, the next most likely mode 2nd, etc., and the least likely mode last. Tip: Click and drag on the mode name.

CONOWALK

Why did you not consider walking for your most recent commute to work?

CONOBIKE

Why did you not consider bicycling for your most recent commute to work?

CONOAUTO

Why did you not consider driving an automobile for your most recent commute to work?

CONOPASS

Why did you not consider riding as a passenger in an automobile for your most recent commute to work?

CONOTRAN

Why did you not consider riding public transit for your most recent commute to work? 
Loop based off question COMODERANK (Displayed Choices, ordered) \{Loop \#, Field 1, Field 2, Field 3, Field 4, Field 5\}

Loop x1: Walking, walking, walked, commuting by walking, Commuting by walking

Loop x2: Bicycling, bicycling, ridden a bicycle, commuting by bicycle, Commuting by bicycling

Loop x3: Automobile, driver (personal car/truck/van/SUV, shared vehicle, Zipcar, car2go, etc.), driving an automobile, driven an automobile, commuting by automobile as a driver, Commuting by bicycle as a driver Loop x4: Automobile, passenger (personal vehicle, carpool, taxi, Uber, Lyft, etc.), riding as a passenger in an automobile, ridden as a passenger in an automobile, commuting by automobile as a passenger, Commuting by automobile as a passenger

Loop x5: Public transit (bus, streetcar, light rail, etc.), riding public transit, ridden public transit, commuting by public transit, Commuting by public transit Loop x6: Other, using some other mode, used some other mode, commuting by some other mode, Commuting by some other mode

QD2TWALK, QD2TBIKE, QD2TAUTO, QD2TTRAN, QD2TOTH Now, imagine that you had $\$\{\mid \mathrm{m}: / /$ Field $/ 3\}$ for your most recent commute to work.

ATAYES

Do you want to answer questions about $\$\{\operatorname{lm://Field} / 4\}$ ?

O Yes

O No

If No Is Selected, Then Skip To End of Block 
COMODEAUTO

What kind of automobile would that have been? (Check all that apply.)

口 Personal vehicle

- Commercial vehicle

- Carpool or vanpool vehicle

Uber

Lyft

- Taxi

Zipcar

car2go

Rental car

Other (please specify)

COMODETRAN

What kind of public transit vehicle would that have been? (Check all that apply.)

TriMet bus

C C-TRAN bus

Portland Streetcar

MAX light rail

WES commuter rail

P Portland Aerial Tram

$\square$ Other (please specify)

COMODEOTH

Would you have used any other transportation modes on this commute trip? (Check all that apply, or No other modes.) Include modes used to get to and from public transit, or to get from a parking space to your workplace.

$\square$ Walking (1)

Bicycling (2)

- Automobile, driver (personal car/truck/van/SUV, shared vehicle, Zipcar, car2go, etc.) (3)

Automobile, passenger (personal vehicle, carpool, taxi, Uber, Lyft, etc.) (4)

Public transit (bus, streetcar, light rail, etc.) (5)

Other (please specify) (90)

No other modes (99) 


\section{ATAPART}

The following questions ask about the things you would have done if you had $\$\{/ \mathrm{m}: / /$ Field/3\} on your most recent commute to work. Think about everything you would have done after leaving your home until arriving at work. For instance, consider what you would have done while on board, getting to/from, and waiting for public transit.

While $\$\{\mid \mathrm{m}: / /$ Field $/ 2\}$, would you have done any of the following things? (Check all that apply, or Doing nothing.)

T Talking face-to-face with people you know

Talking face-to-face with strangers

$\square$ Talking on the phone

Texting, emailing, or other messaging

Reading print (newspaper, book, etc.)

Reading electronically (e-book, website, etc.)

Writing or editing paper documents

Writing or editing electronic documents

Listening to music, radio, or other audio

Watching movie, TV, or other video

Using social websites or apps (Facebook, Twitter, Linkedln, Tumblr, Instagram, etc.)

- Playing game (Pokémon Go, puzzle, etc.)

E Eating food; drinking beverage

Smoking or vaping

Personal grooming (shaving, makeup, etc.)

Caring for children or pets (dog walking, etc.)

S Singing; dancing

Exercising or being physically active

- Planning or navigating this trip

V Viewing scenery; watching people

Thinking or daydreaming

- Sleeping or snoozing

Doing nothing

Other (please specify)

For each selected in ATAPART

ATADUR

While $\$\{\mathrm{Im}: / /$ Field $/ 2\}$, approximately what percentage of your commute travel time would you have spent doing the following things? Your totals may add up to more than $100 \%$ if you would have done two or more things at once.

Percentage (\%): 0, 10, 20, 30, 40, 50, 60, 70, 80, 90, 100, Don't know 
ATAUSE

In terms of its value to you, overall, how useful would you rate the time you would have spent $\$\{\mathbf{I m}: / /$ Field/2\}? Ignore the value of getting to your destination, and think only about the things you would have done while $\$\{l \mathrm{~m}: / /$ Field $/ 2\}$ and the time you would have spent doing them.

O Mostly wasted

Somewhat wasted

O Neither wasted nor useful

O Somewhat useful

O Mostly useful

\section{ATEPNA}

The following questions ask about your feelings and experiences as if you had $\$\{\mid m: / / F i e l d / 3\}$ on your most recent commute to work. Think about everything you would have felt and experienced after leaving your home until arriving at work. For instance, consider what you would have experienced while on board, getting to/from, and waiting for public transit.

Thinking about yourself and your most recent commute to work, would you have felt any of the following, at least a little, while $\$\{1 \mathrm{~m}: / /$ Field/2\}? (Check all that apply, or None of the above.)

Upset
$\square$ Hostile
Alert
Ashamed
$\square$ Inspired
Nervous
$\square$ Determined
Attentive
Afraid
$\square$ Active
$\square$ None of the above




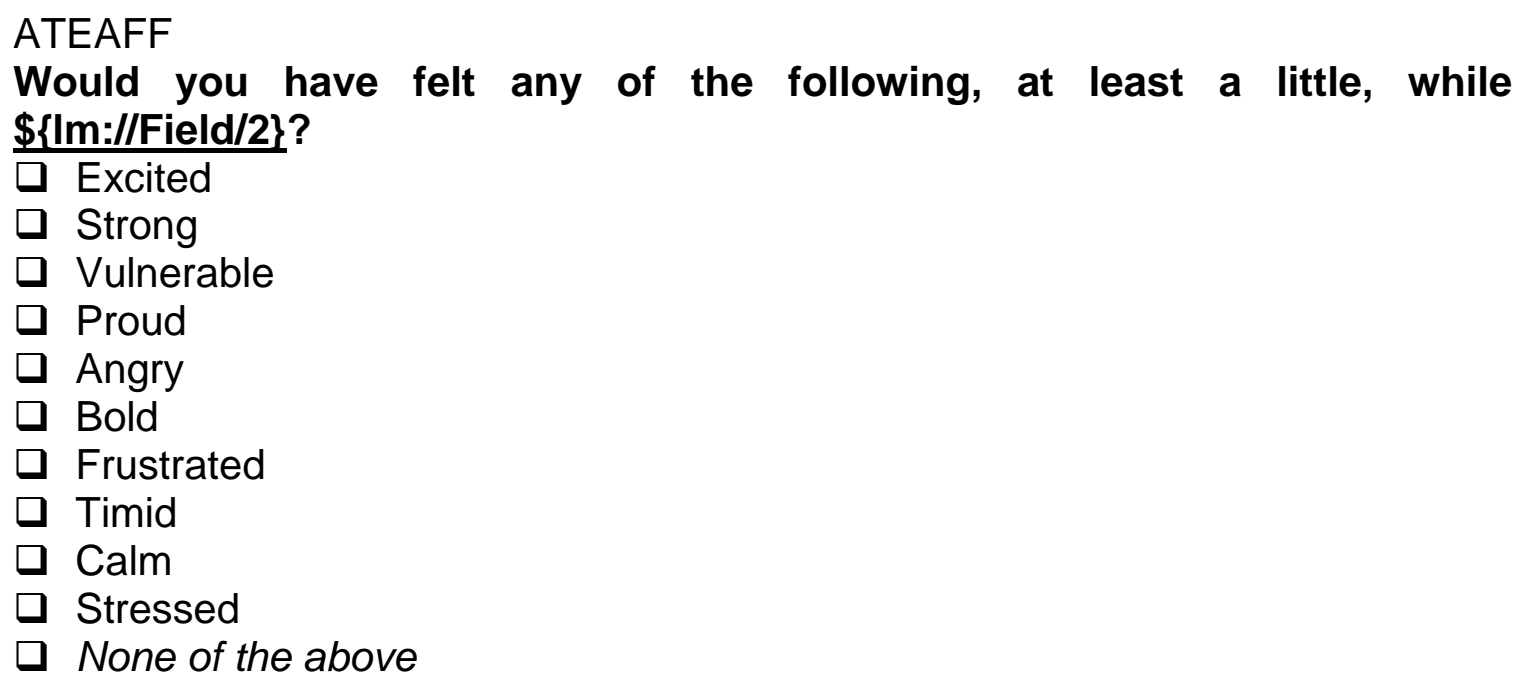

ATESEN

Would you have felt any of the following, at least a little, while $\$\{\mid \mathrm{m}: / /$ Field $/ 2\}$ ? a Hot

Cold

Wet

Sore

Dirty

Sweaty

None of the above 


\section{ATESTS}

For each of the following pairs, select the choice that best corresponds to what your overall experience would have been $\$\{\mid \mathrm{m}: / /$ Field/2\} on your most recent commute to work. For example: If you would have been very tense, select the leftmost choice. If you would have been neither tense nor relaxed, select the middle or neutral choice.

\begin{tabular}{|c|c|c|c|c|c|c|c|c|}
\hline & $\leftarrow$ & & & neutral & & & $\rightarrow$ & \\
\hline $\begin{array}{l}\text { I would have been } \\
\text { very tense. }\end{array}$ & 0 & 0 & 0 & 0 & 0 & 0 & 0 & $\begin{array}{l}\text { I would have been } \\
\text { very relaxed. }\end{array}$ \\
\hline & 0 & 0 & 0 & 0 & 0 & 0 & 0 & $\begin{array}{l}\text { I would have been } \\
\text { very enthusiastic. }\end{array}$ \\
\hline $\begin{array}{l}\text { I would have been } \\
\text { very sad. }\end{array}$ & 0 & 0 & O & 0 & 0 & 0 & 0 & $\begin{array}{l}\text { I would have been } \\
\text { very happy. }\end{array}$ \\
\hline & 0 & 0 & 0 & O & 0 & 0 & 0 & $\begin{array}{l}\text { I would have been } \\
\text { very energized. }\end{array}$ \\
\hline $\begin{array}{l}\text { I would have been } \\
\text { very distressed. }\end{array}$ & 0 & 0 & 0 & 0 & 0 & 0 & 0 & $\begin{array}{l}\text { I would have been } \\
\text { very content. }\end{array}$ \\
\hline $\begin{array}{l}\text { My trip would have } \\
\text { gone poorly. }\end{array}$ & 0 & 0 & O & 0 & 0 & 0 & O & $\begin{array}{l}\text { My trip would have } \\
\text { gone smoothly. }\end{array}$ \\
\hline $\begin{array}{l}\text { My trip would have } \\
\text { been displeasing. }\end{array}$ & 0 & 0 & O & 0 & 0 & 0 & 0 & $\begin{array}{l}\text { My trip would have } \\
\text { been enjoyable. }\end{array}$ \\
\hline $\begin{array}{l}\text { I would have been } \\
\text { worried I wouldn't } \\
\text { arrive on time. }\end{array}$ & 0 & 0 & O & O & O & 0 & O & $\begin{array}{l}\text { I would have been } \\
\text { confident I would } \\
\text { arrive on time. }\end{array}$ \\
\hline $\begin{array}{l}\text { My trip would have } \\
\text { been the worst I } \\
\text { can imagine. }\end{array}$ & 0 & 0 & 0 & 0 & 0 & 0 & 0 & $\begin{array}{l}\text { My trip would have } \\
\text { been the best I } \\
\text { can imagine. }\end{array}$ \\
\hline
\end{tabular}


ATEDES

For the following questions: check all that apply, or select "None of the above".

Would $\$\{\mid \mathrm{m}: / /$ Field/2\} have allowed you, at least a little, to fulfill your desire for:

$\square$ Variety

Control

a Adventure

Companionship

- Freedom

Privacy

$\square$ Safety

C Comfort

Stress relief

A routine

A challenge

A buffer between home and work

Membership in a group or class

None of the above

ATEEXP

Would $\$\{\mathrm{Im}: / /$ Field $/ 2\}$ have allowed you, at least a little, to express your:

a Independence

Social status

Self-identity

Courage

Mastery of a skill

Environmental values

None of the above

ATEIMP

Would $\$\{\mathrm{Im}: / /$ Field $/ 2\}$ have allowed you, at least a little, to improve your:

$\square$ Self-confidence

Mental health

Physical health

None of the above 


\section{ATELIKE}

Overall, how much would you have liked $\$\{\mid \mathrm{m}: / /$ Field/2\} on your most recent commute to work?

O Strongly dislike

O Somewhat dislike

O Neither like nor dislike

O Somewhat like

O Strongly like

DRAWING

Congratulations, you made it to the end!

As a reward, we are offering you the chance to win a prize. Would you like to enter the drawing to win one of ten $\$ 100$ Visa gift cards?

O Yes

O No

\section{CONTACT}

Please provide an email address to enter the drawing.

The information you provide is private and will be treated confidentially. It is being used ONLY for the purposes of this drawing, and it will be deleted immediately after prizes are awarded.

FOLLOWUP

Would you be willing to be contacted by us with follow-up questions related to this survey?

O Yes

O No 
QOPT

We have developed an optional Part II survey that includes additional questions about:

- How you get around using different means of transportation

- Your thoughts on various topic, including multitasking, satisfaction, and attitudes

- Your physical activity levels

The Part II survey will take approximately 15 to 20 minutes to complete. If you complete the Part II survey, you will receive a second entry into the drawing to win one of ten $\$ 100$ Visa gift cards.

OPT1

Are you willing to answer these additional questions?

O Yes

O No

Answer If "Yes" Is Selected

OPT1T

Thank you for your help! You will be redirected to the Part II survey after you submit this survey.

Answer If "No" Is Selected

OPT2

Would you be willing to answer these additional questions at a later time?

O Yes

O No

Answer If "Yes" Is Selected

OPT3

May we email you with a link to these additional questions?

$\mathrm{O}$ Yes

O No

Answer If "Yes" Is Selected

OPT3T1

Thank you for your help! You will receive an email with a link shortly after you submit this survey. 
Answer If "No" Is Selected

OPT3T2

Thank you for your help! Click this link to proceed to the survey, or copy the following URL and save it for another time.

[URL]

ENDTEXT

This is the final question. Is there anything else you would like to tell us?

QSUB

Thank you very much for completing this survey! Please click the Submit button to finish. 


\section{Commuting Survey 2016 - Part II}

\section{QSTART2}

You are being invited to continue your participation in a Portland State University research study about your commuting experiences. The information you provide will be analyzed to better understand transportation and commuting behaviors.

This study is being conducted by Patrick Singleton and Dr. Kelly Clifton, from the Department of Civil \& Environmental Engineering at Portland State University. The research is part of a doctoral dissertation, with funding from the National Institute for Transportation and Communities, a program of the Transportation Research and Education Center for Portland State University.

This Part II survey will take about 15 to 20 minutes to complete. It covers the following topics:

- How you get around using different means of transportation

- Your thoughts about various topics, including multitasking, satisfaction, and attitudes

- Your physical activity levels

There are no known risks if you decide to participate in this research study. There are no costs to you for participating in the study. There are no direct benefits to you for participating in the study. However, the information learned in this study may provide more general benefits to society, such as increased insight into how and why people commute in different ways.

If you complete the Part II survey, you will receive a second entry into the drawing to win one of ten $\$ 100$ Visa gift cards. You will be asked to provide an email address, which will be deleted after prizes are awarded.

If you have any questions about the study, please email tstudy@pdx.edu, call 503893-9677, or write to: Patrick Singleton \& Dr. Kelly Clifton, Portland State University, Civil \& Environmental Engineering, PO Box 751 - CEE, Portland, OR 97207-0751. The Portland State University Institutional Review Board has reviewed this project. If you have any concerns about your rights in this study, please contact the PSU Office of Research Integrity at 503-725-2227 or email hsrrc@pdx.edu. 
This survey is anonymous, although you may be asked to provide some indirectlyidentifying information (demographics, home and job location). This personal information will be treated confidentially. No one will attempt to identify you or your answers, and no one will know whether or not you participated in the study. Your employer will not have access to your responses. Individuals from the Institutional Review Board may inspect these records. Should the data be published, no individual information will be disclosed.

Your participation in this study is voluntary. You may decline to answer a particular question by selecting "Prefer not to answer" or skipping the question. You may exit the survey at any time by closing the survey window or tab. By starting this survey, you are voluntarily agreeing to participate.

By clicking "Accept" at the end of this page, you are consenting to participate in this survey. If you do not consent, please click "Decline" to navigate away from the survey.

CONSENT2

Please indicate your consent to participate in this survey.

O Accept

O Decline

\section{CONTACT2}

Please provide your email address.

The information you provide is private and will be treated confidentially. It is being used ONLY to link your responses in this survey to your previous responses, and for the purposes of the drawing. It will be deleted immediately after prizes are awarded. 
QE

First, we would like to ask you some questions about how you get around in your daily life, specifically your use of different means or modes of transportation. This section will take approximately $\underline{5 \text { minutes }}$ to complete.

For the following questions, consider all forms and instances of personal transportation within the region in which you live (up to about 60 miles). Think about all the times you leave your home for any reason, whether to visit a neighbor, go for a walk, or shop across town. Do not consider times when you go on vacation or travel overnight for work.

\section{MFREQ1}

At this time of year, which of the following transportation modes do you use at least once a week, on average? (Check all that apply.) Consider even short trips, like going around the block or getting to/from public transit.

W Walking

Bicycling

- Automobile, driver (personal car/truck/van/SUV, shared vehicle, Zipcar, car2go, etc.)

Automobile, passenger (personal vehicle, carpool, taxi, Uber, Lyft, etc.)

Public transit (bus, streetcar, light rail, etc.)

Other (please specify)

\section{MFREQ2}

At this time of year, how often do you use the following transportation modes, on average?

Never (1), Less than once a month (2), About once a month (3), 2-3 times / month (4), Don't know (95)

\begin{tabular}{|l|l|l|l|l|l|}
\hline Walking & $(1)$ & $(2)$ & $(3)$ & $(4)$ & $(95)$ \\
Bicycling & 0 & 0 & 0 & 0 & 0 \\
Automobile, driver & 0 & 0 & 0 & 0 & 0 \\
Automobile, passenger & 0 & 0 & 0 & 0 & 0 \\
Public transit & 0 & 0 & 0 & 0 & 0 \\
\hline
\end{tabular}




\section{MFREQ3}

At this time of year, how often do you use the following transportation modes, on average?

About once a week (5), 2-3 days / week (6), 4-5 days / week (7), Almost every day (8), Don't know (95)

\begin{tabular}{|l|l|l|l|l|l|}
\hline Walking & $(5)$ & $(6)$ & $(7)$ & $(8)$ & $(95)$ \\
Bicycling & 0 & 0 & 0 & 0 & 0 \\
Automobile, driver & 0 & 0 & 0 & 0 & 0 \\
Automobile, passenger & 0 & 0 & 0 & 0 & 0 \\
Public transit & 0 & 0 & 0 & 0 & 0 \\
Other & 0 & 0 & 0 & 0 & 0 \\
\hline
\end{tabular}

\section{MFREQ4}

Would you like to use the following transportation modes less, the same, or more than you currently do?

Much less (1), Somewhat less (2), About the same (3), Somewhat more (4), Much more (5)

\begin{tabular}{|l|l|l|l|l|l|}
\hline Walking & $(1)$ & $(2)$ & $(3)$ & $(4)$ & $(5)$ \\
Bicycling & 0 & 0 & 0 & 0 & 0 \\
Automobile, driver & 0 & 0 & 0 & 0 & 0 \\
Automobile, passenger & 0 & 0 & 0 & 0 & 0 \\
Public transit & 0 & 0 & 0 & 0 & 0 \\
Other & 0 & 0 & 0 & 0 & 0 \\
\hline
\end{tabular}


Loop based off question MFREQ1 (Selected Choices)

\{Loop \#, Field 1, Field 2, Field 3\}

Loop 1: Walking, walking, walk

Loop 2: Bicycling, bicycling, ride a bicycle

Loop 3: Automobile, driver, driving an automobile

Loop 4: Automobile, passenger, riding as a passenger in an automobile

Loop 5: Public transit, riding public transit

Loop 6: Other, using some other mode, use some other mode

QETWALK, QETBIKE, QETAUTO, QETTRAN, QETOTH

You reported $\$\{\mid \mathrm{m}: / /$ Field $/ 2\}$ at least once a week. In answering the following questions, think about all the times you $\$\{\mathrm{Im}: / /$ Field $/ 3\}$ to get around within your region, even for a short distance.

MPURP

In your daily life, do you ever $\$\{\mathrm{Im}: / /$ Field $/ 3\}$ to go do the following things or for the following reasons? (Check all that apply.)

W Work

School

Grocery shopping

O Other shopping

- Household errands, personal business, and health care (bank, cleaners, doctor, dentist, etc.)

Civic and religious activities

Eating or drinking outside of home (restaurant, bar, etc.)

Taking other people places (to school, etc.)

- Indoor entertainment and recreation (movie, museum, gym, indoor concert, etc.)

Outdoor entertainment and recreation, in one location (sports, other athletics, outdoor festival, etc.)

- Exercise as transportation, returning to your starting location (going for a walk, jogging, recreational bicycling, etc.)

V Visiting friends and family

Other (please specify) 


\section{MPERC}

For each of the following pairs, select the choice that best corresponds to your overall impression of $\$\{\mathbf{I m}: / /$ Field $/ \mathbf{2}\}$. For example: If you think $\$\{\mathrm{Im}: / /$ Field $/ 2\}$ is very slow, select the leftmost choice. If you think it is very fast, select the rightmost choice. If think it is neither slow nor fast, select the middle or neutral choice.

\begin{tabular}{|c|c|c|c|c|c|c|}
\hline & $\leftarrow$ & & neutral & & $\rightarrow$ & \\
\hline Slow & $\mathrm{O}$ & 0 & $\mathrm{O}$ & 0 & O & Fast \\
\hline Expensive & $\mathrm{O}$ & O & O & O & $\mathrm{O}$ & Affordable \\
\hline Inconvenient & $\mathrm{O}$ & O & $\mathrm{O}$ & O & O & Convenient \\
\hline Unpredictable & $\mathrm{O}$ & O & $\mathrm{O}$ & O & $\mathrm{O}$ & Reliable \\
\hline Risky & O & O & $\mathrm{O}$ & O & $\mathrm{O}$ & $\begin{array}{l}\text { Safe (from traffic } \\
\text { collisions and injuries) }\end{array}$ \\
\hline Vulnerable & O & O & $\mathrm{O}$ & O & $\mathrm{O}$ & $\begin{array}{l}\text { Secure (from crime or } \\
\text { violence) }\end{array}$ \\
\hline Unhealthy for me & $\mathrm{O}$ & 0 & $\mathrm{O}$ & 0 & $\mathrm{O}$ & Healthy for me \\
\hline Harms the environment & $\mathrm{O}$ & O & $\mathrm{O}$ & O & O & Helps the environment \\
\hline A waste of time & $\mathrm{O}$ & 0 & 0 & O & $\mathrm{O}$ & A good use of time \\
\hline Uncomfortable & $\mathrm{O}$ & O & $\mathrm{O}$ & O & O & Comfortable \\
\hline Boring & $\mathrm{O}$ & O & $\mathrm{O}$ & O & O & Fun \\
\hline
\end{tabular}

QF

Thank you for your responses!

Now, we would like to ask you about your thoughts on a number of topics:

- Multitasking and perceptions of time

- Feelings and satisfaction with your life and your job

- Attitudes about technology, transportation, the environment, and health This section will take approximately 10 to 15 minutes to complete. 
MPI

First, consider multitasking: doing more than one thing at the same time. Think about what you do in all aspects of your life, including at home, at work, or while relaxing. Tasks can be anything from an assignment or project to cooking dinner or watching television.

Please state how much you agree with the following statements.

Strongly disagree (1), Somewhat disagree (2), Neither agree nor disagree (3), Somewhat agree (4), Strongly agree (5)

\begin{tabular}{|c|c|c|c|c|c|}
\hline & (1) & (2) & (3) & (4) & (5) \\
\hline $\begin{array}{l}\text { I prefer to work on several tasks in a day, } \\
\text { rather than completing one task and then } \\
\text { switching to another. }\end{array}$ & 0 & O & 0 & 0 & 0 \\
\hline $\begin{array}{l}\text { I would like to work in a job where I was } \\
\text { constantly shifting from one task to another, } \\
\text { like a receptionist or an air traffic controller. }\end{array}$ & 0 & O & 0 & 0 & 0 \\
\hline $\begin{array}{l}\text { I lose interest in what I am doing if I have to } \\
\text { focus on the same task for long periods of } \\
\text { time, without thinking about or doing } \\
\text { something else. }\end{array}$ & $\mathrm{O}$ & O & 0 & 0 & 0 \\
\hline $\begin{array}{l}\text { When doing a number of assignments, I like } \\
\text { to switch back and forth between them rather } \\
\text { than do one at a time. }\end{array}$ & 0 & O & 0 & 0 & 0 \\
\hline $\begin{array}{l}\text { I like to finish one task completely before } \\
\text { focusing on anything else. }\end{array}$ & 0 & O & 0 & 0 & 0 \\
\hline $\begin{array}{l}\text { It makes me uncomfortable when I am not } \\
\text { able to finish one task completely before } \\
\text { focusing on another task. }\end{array}$ & 0 & 0 & 0 & 0 & 0 \\
\hline $\begin{array}{l}\text { I am much more engaged in what I am doing } \\
\text { if I am able to switch between several } \\
\text { different tasks. }\end{array}$ & 0 & O & 0 & 0 & 0 \\
\hline $\begin{array}{l}\text { I do not like having to shift my attention } \\
\text { between multiple tasks. }\end{array}$ & 0 & O & 0 & 0 & 0 \\
\hline $\begin{array}{l}\text { I would rather switch back and forth between } \\
\text { several tasks than concentrate my efforts on } \\
\text { just one. }\end{array}$ & 0 & O & 0 & 0 & 0 \\
\hline $\begin{array}{l}\text { I would prefer to work in an environment } \\
\text { where I can finish one task before starting } \\
\text { the next. }\end{array}$ & 0 & O & 0 & 0 & 0 \\
\hline $\begin{array}{l}\text { I don't like when I have to stop in the middle } \\
\text { of a task to work on something else. }\end{array}$ & O & O & 0 & $\mathrm{O}$ & 0 \\
\hline
\end{tabular}


When I have a task to complete, I like to break it up by switching to other tasks intermittently.

I have a "one-track" mind.

I prefer not to be interrupted when working on a task.

\begin{tabular}{l|l|l|l|l|}
0 & 0 & 0 & 0 & 0 \\
0 & 0 & 0 & 0 & 0 \\
0 & 0 & 0 & 0 & 0 \\
\hline
\end{tabular}

\section{TUSE}

Next, consider your perceptions of time. Think about how you spend your time engaged in daily activities, whether at home, at work, or elsewhere. Please state how much you agree with the following statements.

Strongly disagree (1), Somewhat disagree (2), Neither agree nor disagree (3), Somewhat agree (4), Strongly agree (5)

\begin{tabular}{|c|c|c|c|c|c|}
\hline & (1) & (2) & (3) & (4) & (5) \\
\hline $\begin{array}{l}\text { I usually have plenty of time in my day to } \\
\text { accomplish what I want to accomplish. }\end{array}$ & $\mathrm{O}$ & $\mathrm{O}$ & O & O & $\mathrm{O}$ \\
\hline $\begin{array}{l}\text { I'm often bored and have trouble figuring out } \\
\text { what to do during the day. }\end{array}$ & O & $\mathrm{O}$ & O & O & $\mathrm{O}$ \\
\hline I usually feel rushed. & O & $\mathrm{O}$ & O & O & O \\
\hline $\begin{array}{l}\text { If something l'm doing runs late, it's no big } \\
\text { deal. }\end{array}$ & O & O & O & O & $\mathrm{O}$ \\
\hline I'm often late to my appointments. & 0 & 0 & 0 & 0 & 0 \\
\hline $\begin{array}{l}\text { I usually show up early or on-time to my } \\
\text { appointments. }\end{array}$ & O & $\mathrm{O}$ & O & $\mathrm{O}$ & $\mathrm{O}$ \\
\hline I usually have plenty of free time in my day. & $\mathrm{O}$ & $\mathrm{O}$ & O & O & $\mathrm{O}$ \\
\hline I have almost no time to do with as I wish. & O & $\mathrm{O}$ & O & $\mathrm{O}$ & $\mathrm{O}$ \\
\hline $\begin{array}{l}\text { If I had another hour every day, l'd spend it } \\
\text { working or doing chores. }\end{array}$ & O & O & O & $\mathrm{O}$ & O \\
\hline $\begin{array}{l}\text { If I had another hour every day, l'd spend it } \\
\text { doing something fun. }\end{array}$ & $\mathrm{O}$ & $\mathrm{O}$ & O & $\mathrm{O}$ & 0 \\
\hline $\begin{array}{l}\text { If I had another hour every day, l'd spend it } \\
\text { doing something helpful or meaningful. }\end{array}$ & O & 0 & 0 & 0 & 0 \\
\hline $\begin{array}{l}\text { I'd rather not have another hour every day; } \\
\text { the day is too long already! }\end{array}$ & 0 & 0 & 0 & 0 & 0 \\
\hline
\end{tabular}




\section{PANAS}

Now, consider the things you commonly feel throughout your life. Thinking about yourself and how you normally feel on average, to what extent do you generally feel:

Never (1), Sometimes (2), About half the time (3), Most of the time (4), Always (5)

\begin{tabular}{l|l|l|l|l|l|}
\hline Upset & $(1)$ & $(2)$ & $(3)$ & $(4)$ & $(5)$ \\
Hostile & 0 & 0 & 0 & 0 & 0 \\
Alert & 0 & 0 & 0 & 0 & 0 \\
Ashamed & 0 & 0 & 0 & 0 & 0 \\
Inspired & 0 & 0 & 0 & 0 & 0 \\
Nervous & 0 & 0 & 0 & 0 & 0 \\
Determined & 0 & 0 & 0 & 0 & 0 \\
Attentive & 0 & 0 & 0 & 0 & 0 \\
Afraid & 0 & 0 & 0 & 0 & 0 \\
Active & 0 & 0 & 0 & 0 & 0 \\
\hline
\end{tabular}




\section{SWLFS}

Now, consider your overall satisfaction with your life. Indicate your agreement with the following statements with which you may agree or disagree. Please be open and honest in your responding.

Strongly disagree (1), Somewhat disagree (2), Neither agree nor disagree (3), Somewhat agree (4), Strongly agree (5)

\begin{tabular}{|c|c|c|c|c|c|}
\hline & (1) & (2) & (3) & (4) & (5) \\
\hline In most ways my life is close to ideal. & O & O & O & O & O \\
\hline The conditions of my life are excellent. & $\mathrm{O}$ & 0 & 0 & $\mathrm{O}$ & O \\
\hline I am satisfied with my life. & O & 0 & 0 & $\mathrm{O}$ & O \\
\hline $\begin{array}{l}\text { So far I have gotten the important things I } \\
\text { want out of life. }\end{array}$ & O & $\mathrm{O}$ & O & $\mathrm{O}$ & O \\
\hline $\begin{array}{l}\text { If I could live my life over, I would change } \\
\text { almost nothing. }\end{array}$ & O & O & O & $\mathrm{O}$ & O \\
\hline I lead a purposeful and meaningful life. & O & 0 & 0 & 0 & O \\
\hline $\begin{array}{l}\text { My social relationships are supportive and } \\
\text { rewarding. }\end{array}$ & $\mathrm{O}$ & O & O & $\mathrm{O}$ & 0 \\
\hline $\begin{array}{l}\text { I am engaged and interested in my daily } \\
\text { activities. }\end{array}$ & O & O & O & O & O \\
\hline $\begin{array}{l}\text { I actively contribute to the happiness and } \\
\text { well-being of others. }\end{array}$ & O & 0 & $\mathrm{O}$ & 0 & $\mathrm{O}$ \\
\hline $\begin{array}{l}\text { I am competent and capable in the activities } \\
\text { that are important to me. }\end{array}$ & O & 0 & 0 & 0 & 0 \\
\hline I am a good person and live a good life. & O & O & O & $\mathrm{O}$ & $\mathrm{O}$ \\
\hline I am optimistic about my future. & $\mathrm{O}$ & O & O & O & $\mathrm{O}$ \\
\hline People respect me. & O & O & O & O & 0 \\
\hline
\end{tabular}


BIAJS

Thinking specifically about your current job, do you agree with the following?

Strongly disagree (1), Somewhat disagree (2), Neither agree nor disagree (3), Somewhat agree (4), Strongly agree (5)

\begin{tabular}{|l|l|l|l|l|l|}
\hline I find real enjoyment in my job. & $(1)$ & $(2)$ & $(3)$ & $(4)$ & $(5)$ \\
My job is unusual. & 0 & 0 & 0 & 0 & 0 \\
I like my job better than the average person. & 0 & 0 & 0 & 0 & 0 \\
My job needs me to be fit. & 0 & 0 & 0 & 0 & 0 \\
Most days I am enthusiastic about my job. & 0 & 0 & 0 & 0 & 0 \\
My job is time consuming. & 0 & 0 & 0 & 0 & 0 \\
I feel fairly well satisfied with my job. & 0 & 0 & 0 & 0 & 0 \\
\hline
\end{tabular}




\section{MTUAS}

The following questions ask about your attitudes on various topics.

First, consider your attitudes about technology. Please state how much you agree with the following statements.

Strongly disagree (1), Somewhat disagree (2), Neither agree nor disagree (3), Somewhat agree (4), Strongly agree (5)

\begin{tabular}{|c|c|c|c|c|c|}
\hline & (1) & (2) & (3) & (4) & (5) \\
\hline $\begin{array}{l}\text { I feel it is important to be able to find any } \\
\text { information whenever I want online. }\end{array}$ & $\mathrm{O}$ & $\mathrm{O}$ & O & $\mathrm{O}$ & $\mathrm{O}$ \\
\hline $\begin{array}{l}\text { I feel it is important to be able to access the } \\
\text { Internet any time I want. }\end{array}$ & $\mathrm{O}$ & $\mathrm{O}$ & $\mathrm{O}$ & $\mathrm{O}$ & $\mathrm{O}$ \\
\hline $\begin{array}{l}\text { I think it is important to keep up with the } \\
\text { latest trends in technology. }\end{array}$ & 0 & $\mathrm{O}$ & $\mathrm{O}$ & $\mathrm{O}$ & $\mathrm{O}$ \\
\hline I get anxious when I don't have my phone. & $\mathrm{O}$ & $\mathrm{O}$ & O & $\mathrm{O}$ & O \\
\hline $\begin{array}{l}\text { I get anxious when I don't have the Internet } \\
\text { available to me. }\end{array}$ & O & $\mathrm{O}$ & $\mathrm{O}$ & $\mathrm{O}$ & $\mathrm{O}$ \\
\hline I am dependent on my technology. & $\mathrm{O}$ & 0 & O & 0 & $\mathrm{O}$ \\
\hline $\begin{array}{l}\text { Technology will provide solutions to many of } \\
\text { our problems. }\end{array}$ & $\mathrm{O}$ & $\mathrm{O}$ & O & $\mathrm{O}$ & $\mathrm{O}$ \\
\hline With technology anything is possible. & 0 & 0 & O & $\mathrm{O}$ & $\mathrm{O}$ \\
\hline $\begin{array}{l}\text { I feel that I get more accomplished because } \\
\text { of technology. }\end{array}$ & $\mathrm{O}$ & $\mathrm{O}$ & O & $\mathrm{O}$ & $\mathrm{O}$ \\
\hline $\begin{array}{l}\text { New technology makes people waste too } \\
\text { much time. }\end{array}$ & $\mathrm{O}$ & $\mathrm{O}$ & $\mathrm{O}$ & $\mathrm{O}$ & $\mathrm{O}$ \\
\hline $\begin{array}{l}\text { New technology makes life more } \\
\text { complicated. }\end{array}$ & 0 & 0 & 0 & 0 & 0 \\
\hline $\begin{array}{l}\text { New technology makes people more } \\
\text { isolated. }\end{array}$ & 0 & 0 & 0 & 0 & 0 \\
\hline
\end{tabular}




\section{ATTT1}

Next, consider your attitudes about transportation. How willing would you be to pay higher taxes and/or fees to support the following?

Very willing (1), Somewhat willing (2), Not at all willing (3)

\begin{tabular}{|l|l|l|l|}
\hline More highways & $(1)$ & $(2)$ & $(3)$ \\
More public transportation & 0 & 0 & 0 \\
More projects to increase walking and & 0 & 0 & 0 \\
bicycling & 0 & 0 & 0 \\
More street maintenance & 0 & 0 & 0 \\
More projects to improve traffic safety & 0 & 0 & 0 \\
\hline
\end{tabular}

\section{ATTT2}

How willing would you be to do the following in order to reduce congestion, improve air quality, and protect the environment?

Very willing (1), Somewhat willing (2), Not at all willing (3)

\begin{tabular}{|l|l|l|l|}
\hline Pay a toll to travel on a less congested road & (1) & (2) & (3) \\
Pay higher gas prices & 0 & 0 & 0 \\
Pay more to use a low- or zero-emissions & 0 & 0 & 0 \\
automobile (e.g., electric, hybrid) & & & 0 \\
Pay higher taxes & 0 & 0 & 0 \\
Limit your automobile use & 0 & 0 & 0 \\
Accept cuts in your standard of living & 0 & 0 & 0 \\
\hline
\end{tabular}


ISSP

Next, consider your attitudes about the environment. Please state how much you agree with the following statements.

Strongly disagree (1), Somewhat disagree (2), Neither agree nor disagree (3), Somewhat agree (4), Strongly agree (5)

\begin{tabular}{|c|c|c|c|c|c|}
\hline & (1) & (2) & (3) & (4) & (5) \\
\hline $\begin{array}{l}\text { Modern science will solve our environmental } \\
\text { problems with little change to our way of life. }\end{array}$ & 0 & O & 0 & 0 & O \\
\hline $\begin{array}{l}\text { We worry too much about the future of the } \\
\text { environment and not enough about prices } \\
\text { and jobs today. }\end{array}$ & 0 & O & 0 & 0 & O \\
\hline $\begin{array}{l}\text { Almost everything we do in modern life } \\
\text { harms the environment. }\end{array}$ & 0 & O & 0 & 0 & 0 \\
\hline $\begin{array}{l}\text { People worry too much about human } \\
\text { progress harming the environment. }\end{array}$ & 0 & O & 0 & 0 & 0 \\
\hline $\begin{array}{l}\text { In order to protect the environment, the } \\
\text { country needs economic growth. }\end{array}$ & 0 & O & 0 & 0 & O \\
\hline $\begin{array}{l}\text { Economic growth always harms the } \\
\text { environment. }\end{array}$ & 0 & O & 0 & 0 & 0 \\
\hline $\begin{array}{l}\text { It is just too difficult for someone like me to } \\
\text { do much about the environment. }\end{array}$ & 0 & O & 0 & 0 & O \\
\hline $\begin{array}{l}\text { I do what is right for the environment, even } \\
\text { when it costs more money or takes more } \\
\text { time. }\end{array}$ & O & O & $\mathrm{O}$ & 0 & 0 \\
\hline
\end{tabular}


HS1

Finally, consider your attitudes about health. Please state how much you agree with the following statements.

Strongly disagree (1), Somewhat disagree (2), Neither agree nor disagree (3), Somewhat agree (4), Strongly agree (5)

\begin{tabular}{|c|c|c|c|c|c|}
\hline & (1) & (2) & (3) & (4) & (5) \\
\hline I do everything I can to stay healthy. & O & O & O & O & O \\
\hline $\begin{array}{l}\text { Living life in best possible health is very } \\
\text { important to me. }\end{array}$ & O & O & 0 & O & 0 \\
\hline I actively try to prevent disease and illness. & O & $\mathrm{O}$ & O & O & $\mathrm{O}$ \\
\hline $\begin{array}{l}\text { Eating right, exercising, and taking } \\
\text { preventative measures will keep me healthy } \\
\text { for life. }\end{array}$ & O & $\mathrm{O}$ & 0 & O & O \\
\hline $\begin{array}{l}\text { My health depends on how well I take care } \\
\text { of myself. }\end{array}$ & 0 & O & 0 & 0 & O \\
\hline I like eating healthy foods. & 0 & $\mathrm{O}$ & 0 & O & $\mathrm{O}$ \\
\hline I like exercising. & 0 & O & 0 & 0 & $\mathrm{O}$ \\
\hline I like going to the doctor. & 0 & 0 & 0 & $\mathrm{O}$ & $\mathrm{O}$ \\
\hline
\end{tabular}

HS2

How important do you think the following behaviors are for your overall health?

Very important (1), Somewhat important (2), Not at all important (3)

\begin{tabular}{|l|l|l|l|}
\hline Eating a diet that is low in fat & $(1)$ & $(2)$ & $(3)$ \\
Eating lots of fruits, vegetables, and grains & 0 & 0 & 0 \\
Drinking plenty of water every day & 0 & 0 & 0 \\
Taking vitamins and mineral supplements & 0 & 0 & 0 \\
regularly & 0 & 0 & 0 \\
Exercising regularly & 0 & 0 & 0 \\
Not smoking cigarettes & 0 & 0 & 0 \\
Not drinking alcohol, or drinking in & 0 & 0 & 0 \\
moderation & & \\
Maintaining a healthy body weight &
\end{tabular}


QG

Thank you for your responses!

Now, we would like to ask you some questions about your physical activity levels.

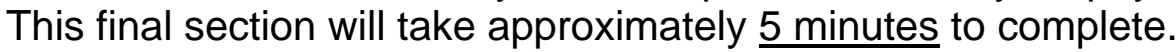

These questions are about how much physical activity you do in a typical week. Please answer even if you do not consider yourself to be a physically-active person. Consider the following definitions:

- Vigorous-intensity activities are those that require hard physical effort and cause large increases in breathing or heart rate.

- Moderate-intensity activities are those that require moderate physical effort and cause small increases in breathing or heart rate.

PAWVIG

Think first about the time you spend doing required tasks as part of your job or your chores at home. Think of the things you have to do such as paid or unpaid work, study/training, and household cleaning or gardening.

Does your job or do your chores involve vigorous-intensity activity that causes large increases in breathing or heart rate for at least 10 minutes continuously? For example: carrying or lifting heavy loads, digging, or construction work.

O Yes

O No

\section{PAWVIGDAY}

In a typical week, on how many days do you do vigorous-intensity activities as part of your job or your chores?

Days per week

PAWVIGTIME

Typically, how much time do you spend doing vigorous-intensity activities as part of your job or your chores on such a day?

\section{Hours}

Minutes

Vigorous-intensity 


\section{PAWMOD}

Does your job or do your chores involve moderate-intensity activity that causes small increases in breathing or heart rate for at least 10 minutes continuously? For example: brisk walking, carrying light loads, waiting tables, or cleaning floors.

O Yes

O No

\section{PAWMODDAY}

In a typical week, on how many days do you do moderate-intensity activities as part of your job or your chores?

Days per week

\section{PAWMODTIME}

Typically, how much time do you spend doing moderate-intensity activities as part of your job or your chores on such a day?

\begin{tabular}{|l|l|l|}
\hline & Hours & Minutes \\
\hline Moderate-intensity & & \\
\hline
\end{tabular}

\section{PAWALKDAY}

The next questions exclude the physical activities as part of your job and your chores that you have already mentioned.

Now think about the usual ways you get to and from places by walking and bicycling. Do not include walking for leisure, bike tours, or cycling for sports.

In a typical week, on how many days do you walk for at least 10 minutes continuously to get to and from places?

Days per week

PAWALKTIME

Typically, how much time do you spend walking on such a day?

\begin{tabular}{|l|l|l|}
\hline & Hours & Minutes \\
\hline
\end{tabular}




\section{PABIKEDAY}

In a typical week, on how many days do you bike for at least 10 minutes continuously to get to and from places? Days per week

PABIKETIME

Typically, how much time do you spend biking on such a day?

\begin{tabular}{|l|l|l|}
\hline & Hours & Minutes \\
\hline Biking & & \\
\hline
\end{tabular}

\section{PALVIG}

The next questions exclude the physical activities as part of your job and your chores and getting to and from places that you have already mentioned.

Now think about sports, fitness, and recreational (leisure) activities, including going for a walk or on a bike tour. These can be outdoor or indoor leisure activities that you do in your free time.

Do you do any vigorous-intensity sports, fitness, or recreational (leisure) activities that cause large increases in breathing or heart rate for at least 10 minutes continuously? For example: running, football, quick pedal cycling, or fitness training.

O Yes

O No

PALVIGDAY

In a typical week, on how many days do you do vigorous-intensity sports, fitness, or recreational (leisure) activities?

Days per week

PALVIGTIME

Typically, how much time do you spend doing vigorous-intensity sports, fitness, or recreational (leisure) activities on such a day?

Vigorous-intensity 


\section{PALMOD}

Do you do any moderate-intensity sports, fitness, or recreational (leisure) activities that cause a small increase in breathing or heart rate for at least 10 minutes continuously? For example: brisk walking, hiking, casual cycling, casual swimming, or gymnastics.

O Yes

O No

\section{PALMODDAY}

In a typical week, on how many days do you do moderate-intensity sports, fitness, or recreational (leisure) activities?

Days per week

\section{PALMODTIME}

Typically, how much time do you spend doing moderate-intensity sports, fitness, or recreational (leisure) activities on such a day?

\begin{tabular}{|l|c|c|}
\hline & Hours & Minutes \\
\hline Moderate-intensity & & \\
\hline
\end{tabular}

\section{PASITTIME}

Finally think about the time you spend sitting or reclining. This could be at your job, at home, getting to and from places, or in your free time. For example: time spent sitting at a desk; eating; traveling in a car, bus, or train; reading; watching television; or using the computer. Time spent sleeping should not be included.

How much time do you usually spend sitting or reclining on a typical day?

\begin{tabular}{|l|l|l|}
\hline & Hours & Minutes \\
\hline Sitting or reclining & & \\
\hline
\end{tabular}

\section{END2TEXT}

This is the final question. Is there anything else you would like to tell us?

\section{QEND2T1}

Thank you very much for completing this survey! You will receive a second entry into the drawing. Please click the Submit button to finish. 